\title{
UNDERSTANDING MENTAL HEALTH AND WELLBEING AMONG ABORIGINAL POST- SECONDARY STUDENTS
}

\author{
by \\ Caitlin Joy Davey \\ Honours Bachelor of Arts, McMaster University, 2009 \\ Master of Arts, Ryerson University, 2011 \\ A dissertation \\ presented to Ryerson University \\ in partial fulfillment of the \\ requirements for the degree of \\ Doctor of Philosophy \\ in the program of \\ Psychology
}

Toronto, Ontario, Canada 2015

CCaitlin Davey 2015 


\section{Author's Declaration}

I hereby declare that I am the sole author of this dissertation. This is a true copy of the dissertation, including any required final revisions, as accepted by my examiners.

I authorize Ryerson University to lend this dissertation to other institutions or individuals for the purpose of scholarly research.

I further authorize Ryerson University to reproduce this dissertation by photocopying or by other means, in total or in part, at the request of other institutions or individuals for the purpose of scholarly research.

I understand that my dissertation may be made electronically available to the public. 


\begin{abstract} Doctor of Philosophy, 2015

Caitlin Joy Davey

Psychology

Ryerson University
\end{abstract}

Understanding mental health and wellbeing knowledge among Aboriginal post-secondary students

First Nations, Inuit and Métis youth in Canada report higher rates of mental health (i.e., depression, anxiety, addiction and suicide) issues than the non-Aboriginal population of youth, which impacts their ability to achieve their educational goals. Understanding mental health from an Aboriginal worldview and through culturally safe strategies designed to translate mental health information to Aboriginal people is lacking. The objectives of the present study were to develop, implement and evaluate a knowledge translation (KT) strategy to enhance wellbeing knowledge and self-efficacy in coping with mental health issues among Aboriginal postsecondary students using a community-based approach. The Tool Development Phase involved focus groups and interviews with Aboriginal post-secondary students $(n=9)$ and community members $(n=3)$ to develop a KT activity. The KT Phase involved a pre/post design where participants $(n=4)$ were asked about their mental health knowledge and self-efficacy in improving their wellbeing prior to and after the KT activity. All students participated in an individual interview one month later about their knowledge and the helpfulness of activity. Key informants $(n=4)$ were interviewed during a Follow-Up and Debriefing phase regarding the community-based approach. Mental health was defined wholistically, as related to community and as being impacted by identity, stigma, cultural connection, intergenerational trauma, and a clash between Aboriginal and positivist Western worldviews. KT preferences included 
incorporating traditional ceremonies; covering particular topics such as resources, traditional teachings and coping strategies; and incorporating active participation. The KT activity increased knowledge and self-efficacy related to mental health and some knowledge was retained and used at one-month follow-up. As well, participants appreciated the sense of community that the activity created and provided feedback regarding what could be changed (e.g., more active participation). Key informants thought the project addressed some community needs and that it was built on relationships. This study will contribute to increasing the wellbeing of Aboriginal students by expanding upon their mental health knowledge, and sense of self-efficacy in coping with their own mental health issues, with the aim of addressing mental health barriers to the completion of post-secondary education among Aboriginal students. 


\section{Acknowledgments}

I want to acknowledge and thank all the Aboriginal students and staff who participated in this study and contributed to understanding mental health and knowledge translation from an Aboriginal worldview. I want to acknowledge Ryerson Aboriginal Student Services for their support and for believing in the project. In particular, I want to thank Ruth Koleszar-Green, Monica McKay, Ed Sackaney, and Joanne Dallaire for making this project possible. I want to thank Nicole Wemigwans and Stephanie Matchiwita for their help and dedication to the data analysis and interpretation. Thank you especially to my incredible supervisor, Dr. Kelly McShane for taking me under her wing and supporting me through such an incredible journey in completing this dissertation. Kelly has been an invaluable source of support through my entire graduate career and it is through her guidance and dedication to my growth and learning that I am able to complete my PhD. Thank you to the Ontario Graduate Scholarship, the Ontario Mental Health Foundation, the Network for Aboriginal Health Research and the Aboriginal Education Council at Ryerson University for providing funding for this project. Thank you to my family for always being so supportive of everything I do. In particular, thank you to my mother, father, sister and godmother: Ellen Brenzil, Gary Davey, Megan Davey, and Susan MacNeil. I want to individually thank my amazing husband, Brandon Lynch for standing by my side through my entire undergraduate and graduate degree with unconditional support for my endeavors. Without all of your support, I wouldn't have had the strength to complete this dissertation or degree. Thank you, everyone! 


\section{Table of Contents}

Introduction $\quad 1-4$

Literature Review 4-40

Aboriginal Peoples in Canada $\quad$ 4-6

Historical Trauma Among First Nations, Inuit and Métis Peoples 5-8

Common Social Adversities Faced by Aboriginal People 8-9

A Focus on Education: Barriers to Attainment $\quad 9-13$

Mental Health among First Nations, Inuit and Métis Youth 13-16

Aboriginal Perspectives on Mental Health 16-19

$\begin{array}{lr}\text { Education as a Way Forward } & 19-20\end{array}$

$\begin{array}{lr}\text { Knowledge Translation in an Aboriginal Context } & 20-27\end{array}$

Community-Based Research 27-30

Relationship with Community Partner 30-31

$\begin{array}{ll}\text { Epistemological Lens } & \text { 31-37 }\end{array}$

$\begin{array}{ll}\text { Thematic Analysis } & \text { 37-38 }\end{array}$

$\begin{array}{lr}\text { Present Study } & \text { 39-39 }\end{array}$

$\begin{array}{ll}\text { Method } & 40-51\end{array}$

$\begin{array}{ll}\text { Overview } & 40-40\end{array}$

$\begin{array}{ll}\text { Participants } & 40-42\end{array}$

$\begin{array}{lr}\text { Materials } & 42-42\end{array}$

$\begin{array}{ll}\text { Design } & 42-43\end{array}$

$\begin{array}{lr}\text { Procedure } & \text { 43-47 }\end{array}$

Qualitative and Quantitative Analysis $\quad$ 48-51 
Results: Tool Development Phase

Definition of Mental Health from an Aboriginal Perspective

Knowledge Translation Activity Content

$82-100$

Knowledge Translation Activity

$100-104$

Results: Knowledge Translation Phase

$105-133$

Activity Evaluation

105-109

Knowledge to Action

$109-125$

Global Messages, Current Challenges, and Ideas for the Future

Results: Follow-Up/Debriefing Phase

$134-183$

Project Impressions

$134-142$

Reflections of the Research Process

$142-151$

Project Roles

$152-156$

Community Partner Needs

$156-161$

Global Messages Received from the Project

$161-165$

Present/Future of Mental Health Knowledge among Aboriginal Students

$165-181$

Project Impacts on Key Informants

$181-183$

Discussion

$184-213$

Tool Development Phase

184-195

Knowledge Translation Phase

195-201

Follow-Up/Debriefing Phase

201-206

Study Strengths

206-208

Study Limitations

208-210

Future Research

210-211 
Table 2. Phase two mental health and wellbeing knowledge uptake and self-efficacy pre and post activity scores by participant $(n=4)$

Appendix C: Student Recruitment Script: Tool Development Phase

Appendix D: Staff Recruitment Script: Tool Development Phase 
Appendix Q: Key Informant Interview Questions

Appendix R: Final Tool Development Phase Codebook

$252-260$

Appendix S: Knowledge Translation Activity Codebooks

$261-270$

Appendix T: Final Follow-Up/Debriefing Phase Codebook

$271-282$

Appendix U: Wellbeing Activity Booklet Handout

283-295

Appendix V: Facilitator's Version of Wellbeing Activity Agenda

296-297

References

298-317 


\section{Personal Introduction}

My name is Caitlin Davey and I am the principle investigator of this dissertation. My spirit name translates to "She Who Digs in the Dirt" in English, which means that I tend to work hard to look beyond the surface to gain understanding. My father is from the Mohawk Nation from Six Nations of the Grand River in Southern Ontario and my mother is Scottish and English and is from Caledonia, Ontario. I identify with all aspects of myself. My clan is turtle who are known for being slow and steady, determined, humbly stubborn and holding a lot on their shoulders. I have spent a lot of time over the years immersing myself into Aboriginal cultures and reconnecting/connecting to the Aboriginal part of me, while acknowledging all aspects of myself. For this dissertation, I used the characteristics described above to understand and write about Aboriginal perspectives on mental health and wellbeing and to design a knowledge translation activity aimed at communicating wellbeing knowledge to Aboriginal post-secondary students.

\section{Introduction}

First Nations, Inuit and Métis youth represent the majority of the Aboriginal ${ }^{1}$ population in Canada and are, therefore, the future of Aboriginal Peoples in Canada (Statistics Canada, 2003). There is much hope that they will be able to move their communities forward, which some suggest will involve educational attainment (Alberta Education, 2002). However, due to the many adversities with which Aboriginal youth are faced, they are at an increased risk of prematurely dropping out of school (Statistics Canada, 2001) and/or not having the opportunity

\footnotetext{
${ }^{1}$ The term "Aboriginal" will be used throughout this dissertation when referring to First Nations, Inuit and Métis populations in Canada. It is uncommon for the literature to specify which Aboriginal group is being referenced and, therefore, the terms used in the references will be used in the present dissertation. When referring to specific Aboriginal groups (e.g., First Nations, Inuit and/or Métis), they will be specified throughout the dissertation.
} 
to complete post-secondary education. One barrier to Aboriginal youth achieving post-secondary education, in particular, is the high risk and prevalence of developing mental health issues (Dell, Hopkins, \& Dell, 2005). First Nations, Inuit and Métis youth suffer from specific social challenges that put them at risk for developing mental health issues (Finlay, Hardy, Morris, \& Nagy, 2010). Aboriginal youth have been found to have high rates of anxiety (Bigfoot \& Schmidt, 2010), depression (The First Nations Information Governance Centre, 2012), substance use issues (The First Nations Information Governance Centre, 2012), and suicide (Health Canada, 2003). Such issues can make it difficult to achieve the requirements necessary for admission to post-secondary institutions and maintaining a good educational standing. Although mental health issues have been recognized as a barrier for post-secondary school achievement among First Nations, Inuit and Métis students, the need for conceptualizing mental health from an Aboriginal worldview is rarely recognized. Therefore, rates of mental health issues that are often presented should be treated with caution, since differing perspectives on mental health from an Aboriginal worldview can affect mental health assessment, as well as treatment outcomes. Without an understanding of mental health from an Aboriginal worldview, postsecondary institutions and the dynamics of these institutions, are at risk of invalidating the mental health struggles and needs of many Aboriginal students.

A consideration of the historical as well as present context of Aboriginal Peoples in Canada in needed to understand mental health among First Nations, Inuit and Métis youth. Specifically, mental health is often viewed wholistically ${ }^{2}$, with a focus on general wellbeing,

\footnotetext{
2 "Wholistic" is spelled this way throughout the manuscript; as such spelling was requested by many Aboriginal students and staff who participated in the study. The word "wholistic" was positioned as better representing wholeness, as "holistic" was viewed as representing the word "hole", which was seen as being in contrast to the meaning of the word.
} 
rather than symptom-focused (Blackstock, 2008). Aboriginal Peoples have also been historically traumatized by the educational system (e.g., residential schools; Indian and Northern Affairs Canada, 1996), which affects the development of mental health issues; as such trauma has been passed on intergenerationally (Connors, 2007). Intergenerational trauma also relates to current adversities that Aboriginal populations face, including but not limited to high rates of poverty, unemployment, lack of access to housing, lack of access to health care and familial violence, which all contribute to the development of mental health issues (Smylie, 2001). I argue that some mental health issues can be alleviated and/or prevented through increasing mental health knowledge and coping strategies, which can be done through knowledge translation (KT). Knowledge translation (KT) in an Aboriginal context is defined as sharing what is known about living a good life (e.g., wellness; Kaplan-Myrth \& Smylie, 2006), which is exactly what the present study aimed to do.

The present study aimed to understand mental health from an Aboriginal worldview as well as Aboriginal post-secondary students' preferences for receiving mental health information in order to effectively translate culturally appropriate mental health information to Aboriginal students. As a result of this study, Aboriginal students can use mental health information, that they learn through this project, in their everyday lives to manage their own mental health and wellbeing. This study used a social constructionist lens as well as community-based approach to research, while also using OCAP (ownership, control, access, and possession) principles. Social constructionism opposes essentialism (i.e., the idea that there is a determined nature to the world or people; Burr, 2003), which is thought to underlie psychological phenomena (e.g., criteria of various mental health concerns as are outlined by the Diagnostic Statistical Manual-IV; American Psychiatric Association, 2000) and assumes that the categories and divisions people 
use on a daily basis are constructed by social products, social institutions and language use (Burr, 2003). Community-based research (CBR) is an approach that emphasizes a collaborative process with the communities involved in research (Cornwall \& Jewkes, 2010; Minkler, 2000). Using primarily qualitative methods, the study sought to develop, implement and evaluate a knowledge translation strategy for Aboriginal post-secondary students to increase their mental health knowledge and coping resources, which in turn, could aid in their successful completion of postsecondary education and improve their ability to move their communities forward.

\section{Literature Review}

\section{Aboriginal Peoples in Canada}

Aboriginal Peoples are the descendants of the first residents of North America. Aboriginal Peoples of Canada include First Nations, Inuit, and Métis People (Constitution Act, 1982). First Nations People may be considered "Status Indian" if they are registered under the Indian Act, but are considered "non-Status Indian" if they are not registered (Isaac, 1995). "Status" means that one has certain rights (e.g., exemption from paying provincial taxes; Hare \& Barman, 2000). A First Nations person may also be considered a "treaty Indian" if one's ancestors signed treaties in Canada and would have certain rights outlined in the numbered treaties that were signed between 1871 and 1922 (Smylie, 2000). Treaty rights include being able to live, hunt, and fish on reserve land (Smylie, 2000). A First Nations person may have both status and treaty rights, or neither (Isaac, 1995; Smylie, 2000). Inuit historically lived above the tree line in Canada. There are currently four Inuit specific regions in Canada. These regions include Nunavut (east of the Northwest Territories), Inuvialuit (western Arctic), Nunavik (northern Quebec), and Nunatsiavut (northern Labrador; Smylie, 2000). The Métis are people whose ancestry comprises First Nations women who married European men in the $17^{\text {th }}$ century (Métis Nation of Ontario, 2010; Smylie, 
2000). It should be noted that there are cultural differences within and between these Aboriginal groups and, therefore, assumptions should never be made regarding a First Nations, Inuit or Métis person's cultural background.

The Aboriginal population represents the fastest growing population in Canada and is growing particularly fast in major cities in Canada such as Toronto, Ontario (Aboriginal Affairs and Northern Development Canada, 2006). First Nations, Inuit and Métis youth represent the majority of the Aboriginal population in Canada (Statistics Canada, 2003). As well, the Aboriginal population in Canada is younger than the non-Aboriginal population. In 2006, 28\% of the Aboriginal population in Canada was under the age of 15 years compared to $17 \%$ of the nonAboriginal population in Canada (Statistics Canada, 2006). Therefore, First Nations, Inuit and Métis youth are the future of the Aboriginal population in Canada; however, their ability to move their communities forward and successfully participate in their community (e.g., through educational attainment) is adversely impacted by the high prevalence of mental health problems that affect First Nations, Inuit and Métis youth (Health Canada, 2003).

In order to understand the prevalence of mental health issues among Aboriginal youth as well as mental health from an Aboriginal worldview, it is important to understand the contextual factors that influence the development of mental health issues among these populations. Since the education system in North America was, in many ways, responsible for traumatizing the Aboriginal population in Canada and influencing the development of mental health issues that have been passed down intergenerationally, it is worth examining this system and how it has historically impacted the rise in prevalence of mental health issues.

\section{Historical Trauma Among First Nations, Inuit and Métis Peoples: A Focus on Education}


Historically, First Nations, Inuit, and Métis People were traumatized through the colonization of Canada (e.g., relocation, the Indian Act, and the education system; Indian and North Affairs Canada, 1996). Recognizing such trauma aids in understanding mental health issues among Aboriginal people in Canada, as historical trauma is one such contextual factor that has been linked to the high prevalence of mental health problems among First Nations, Inuit and Métis populations (McCormick, 2000). During the colonization of Canada, the Canadian Government enacted trauma through attempted assimilation of First Nations, Inuit and Métis populations (Connors, 2007). Residential schools were one of the most influential acts of historical trauma among these populations in Canada and affected all three Aboriginal groups in Canada. Residential schools were created to "civilize" First Nations, Inuit and Métis Peoples (Morgan \& Freeman, 2009) by assimilating these populations to mainstream European society through the education system (i.e., residential schools; Hare \& Barman, 2000). Hundreds of thousands of First Nations, Inuit and Métis children were forced to attend residential schools over more than 100 years (i.e., between 1874 and 1986; Aboriginal Healing Foundation, 2003b).

During their attendance of residential schools, First Nations, Inuit and Métis children were forbidden to practice their cultural traditions in an attempt to eliminate Aboriginal languages, traditions, and beliefs (Indian and Northern Affairs Canada, 1996). Children attending residential schools were also taught to be ashamed of their identity and culture (Aboriginal Healing Foundation, 2003b). If First Nations, Inuit and Métis children spoke their languages or practiced aspects of their culture, they were punished through neglect and physical, emotional and/or sexual abuse (Hare \& Barman, 2000). Such abuse has been found to relate to the development of mental health problems (Aboriginal Healing Foundation, 2007; Brave Heart, 2003). More specifically, Brave Heart (2003) has discussed the "Historical Trauma Response", 
which describes a variety of reactions to historical trauma that may include depression, suicidal ideation and attempts, anxiety, emotional instability and self-destruction. These issues are, in many ways, passed down from generation to generation, which has been described as intergenerational trauma (Brave Heart, 2003). Intergenerational trauma is the unintentional diffusion of trauma to First Nations, Inuit and Métis individuals throughout the generations (Brave Heart, 2003). The effects of such trauma can be demonstrated through the development of mental health issues.

Connors (2007) suggests that the negative impact of historical trauma on the mental health of First Nations, Inuit and Métis populations relates to a lack of coping strategies among Aboriginal populations. First Nations, Inuit and Métis culture has been fragmented by the colonization of Canada and assimilative acts of the Canadian government (e.g., residential schools) and such a lack of culture can lead to poor/absence of coping strategies (Connors, 2007). Culture helps individuals manage difficult circumstances, as people use their cultural teachings to guide them in their ability to manage adversities; however, for many First Nations, Inuit and Métis people, such culture has been taken away (Connors, 2007). Since many First Nations, Inuit and Métis communities are faced with adverse social determinants of health (e.g., poverty, unemployment, and inadequate dwellings; Finlay et al., 2010), which will be further discussed, ineffective coping strategies are exacerbated, contributing to the development of mental health problems. Ineffective coping strategies that are due to a lack of culture are passed down to the next generation (Brave Heart, 2003), influencing the development of mental health issues in First Nations, Inuit and Métis youth.

One of the many consequences of historical trauma is a clash of Aboriginal worldviews and European ways of life, or what has been termed "jagged worldviews" (Little Bear, 2000). 
Such "jagged worldviews" are passed on intergenerationally and contribute to a lack of culture for Aboriginal people to use in creating a cohesive identity, where one's Aboriginal and nonAboriginal identity is unable to merge or become integrated in a way that makes sense for this population (Connors, 2007). There is suggestion that the clash of worldviews, which has stemmed from historical trauma, is at the heart of many issues among Aboriginal Peoples, one of which includes the high prevalence of mental health issues (Battiste, 2000; McCormick, 2000). Such historical trauma has contributed to various social adversities that Aboriginal people face, presently, which can make it difficult for these populations to move their communities forward.

\section{Common Social Adversities Faced by Aboriginal People}

Stemming from the traumatic historical context from which First Nations, Inuit and Métis Peoples come, Aboriginal people face many social adversities that further contribute to mental health issues. According to the First Nations Regional Health Survey (2008/2010; The First Nations Information Governance Centre, 2012), 40.4\% of First Nations adults reported finding it difficult to provide food for their families. As well, 32.2\% reported having difficulty paying for utilities, $26.2 \%$ reported having difficulty paying for clothing, and $16 \%$ reported difficulty finding housing (The First Nations Information Governance Centre, 2012). As well, 60\% of First Nations adults reported an annual income of less than $\$ 20,000$ and, therefore, the majority of this population is living below the poverty line (The First Nations Information Governance Centre, 2012). In addition to First Nations people, Métis and Inuit populations across Canada were found to have an average income of $\$ 22,213$ and $\$ 19,878$, respectively, in 2001 as cited by the Aboriginal Peoples Survey (Statistics Canada, 2001). To make circumstances even more difficult, First Nations, Inuit and Métis people often have difficulty achieving employment status (Smylie, 2001). As well, due to geographic isolation that many First Nations people may 
experience, many do not have access to proper healthcare (The First Nations Information Governance Centre, 2012). Regarding Métis and Inuit populations, they may also be geographically isolated and, in addition to First Nations people, they may not have access to culturally safe health resources (Health Canada, 2010; Nelson, 2012). Rates of housing issues also tend to be higher among many Aboriginal communities. More specifically, according to the First Nations Regional Health Survey, 35.8\% of First Nations adults reported not having basic amenities in their home, including a lack of hot and cold water, flushing toilets, or safe drinking water (The First Nations Information Governance Centre, 2012). Aboriginal people may also experience familial violence, as this has been identified as an increasing problem in many Aboriginal communities (this will vary from family to family and from community to community), as this affects offspring development and the dynamics of the family (Aboriginal Healing Foundation, 2003a).

Although this is not an exhaustive list of social adversities that Aboriginal people face, the final issue of discussion will be the lack of educational attainment, which is very much related to the other social hardships discussed above. The historical trauma faced by Aboriginal Peoples, financial barriers, housing barriers, the lack of access to healthcare, familial violence, and the lack of access and achievement of education contribute to one another in the development or risk of developing a mental health concern among Aboriginal youth (World Health Organization, 2011). These adversities will ultimately affect the ability of Aboriginal people in moving their communities toward self-determination (Asch, 2002).

\section{A Focus on Education: Barriers to Attainment}

The current education system has progressed since the time when residential schools were the norm in Canadian society; however, it continues to traumatize First Nations, Inuit and 
Métis children and youth, creating significant barriers to educational attainment, which contributes to education as a social adversity. Since education is such a large part of children's lives, the development of self-esteem and a sense of self commonly develop during school years. Aboriginal children have been recognized as having the lowest levels of academic achievement as related to academic school grades and graduation rate, over and above all other ethno-cultural groups (Duffy, 1988; Frideres, 1988). Systematic issues, as related to the education system, create difficulties in academic performance and stunt the development of a sense of self and selfesteem for First Nations, Inuit and Métis youth. For example, Inuit students often experience a switch from their heritage to dominant language instruction in the classroom. Bougie, Wright and Taylor (2003) found that Inuit children in grade three, who switched from their heritage to the dominant language in the classroom, had significantly lower self-esteem than those who did not switch languages or those who had dual-heritage languages (i.e., spoke heritage and dominant languages at home). Such children are placed at a disadvantage regarding the development of a positive self-concept and self-esteem, in addition to academic success, as a switch in languages would suddenly create an environment full of confusion, as these children will not understand lessons or instructions. A switch in languages could also invalidate children's heritage languages and can negatively impact identity development, since these languages are considered to be a very important part of First Nations, Inuit and Métis identity (Battiste, 2000).

First Nations and Métis children in the Western educational system experience similar difficulties to Inuit, as they will often experience a language change and their culture, historical context, and unique learning styles are rarely recognized (Alberta Teacher's Association, 2006). On-reserve First Nations children may attend elementary school in their community, but are often required to attend high school, college, and/or University off-reserve in the mainstream 
school system (Alberta Teacher's Association, 2006). Such a transition can be experienced as "culture shock" as well as invalidating to the individual's culture (Alberta Teacher's Association, 2006).

In addition to the barriers faced at the primary and secondary school level, there are additional barriers for Aboriginal people at the post-secondary level, including alternative learning styles that are often not addressed, geographic obstacles when Aboriginal students have to move away from their communities to attend post-secondary institutions, and financial barriers. As well, Aboriginal students face hurdles when they are not prepared academically, as this can affect their transition into a post-secondary institution. There is also a lack of role models in post-secondary institutions for Aboriginal students to look up to and to help them understand what they are capable of accomplishing. There are also discriminatory, language, and curriculum barriers. In particular, curriculum barriers occur when there is a lack of relevant content to Aboriginal students in post-secondary course curricula (Stone Circle, 2011). Although this is not an exhaustive list, a final obstacle for Aboriginal students to completing postsecondary education that will be discussed relates to personal barriers. Personal barriers are in references to the fact that Aboriginal students are often older than non-Aboriginal students, having and average age of 22 years and, therefore, are more likely to have additional responsibilities such as taking care of family and/or children and, therefore, the lack of accommodation by many post-secondary institutions can create significant hurdles for Aboriginal students to overcome to complete their education (Holmes, 2006). Related to increased responsibilities are personal barriers that include stress, lack of housing, issues with childcare and, of course, the development and/or management of mental health issues (Holmes, 2006). 
Due to the disadvantages that First Nations, Inuit and Métis youth currently face within the education system, it is not surprising that the Aboriginal population in Canada has lower educational attainment than the non-Aboriginal population (Statistics Canada, 2001). According to the Aboriginal Peoples Survey (2001), among Aboriginal people ages 20 through 24 years, $48 \%$ have attained less education than high school in comparison to $38 \%$ of the non-Aboriginal population in Canada (Statistics Canada, 2001). As well, 4\% of Aboriginal people in Canada have achieved a University education, while $15 \%$ of the non-Aboriginal population have achieved University education (Statistics Canada, 2001). Rates are more comparable when examining non-University post-secondary education (e.g., college diplomas), where $25 \%$ of the Aboriginal population has achieved this education level, while $28 \%$ of the non-Aboriginal population has achieved this level of education (Statistics Canada, 2001). Although educational attainment rates are lower for Aboriginal people in comparison to the non-Aboriginal population, they have increased from 1996 to 2001 (Statistics Canada, 2001).

It is argued that providing mental health knowledge and tools to Aboriginal postsecondary students can aid in maintaining and increasing educational attainment among Aboriginal students, since it would address some of the personal barriers outlined for Aboriginal students (Holmes, 2006). Such mental health knowledge can help Aboriginal students develop resources (both internal and external) to better manage any mental health issues and to increase their likelihood of successfully achieving their educational goals. As previously discussed, Aboriginal youth are often exposed to many adverse social determinants of health as related to historical and present-day trauma and barriers, which influences the development of mental health issues among these individuals. Therefore, many Aboriginal youth that participate in the educational system may experience mental health issues (R. Koleszar-Green personal 
communication, April 15, 2012). Creating a space where an understanding of Aboriginal worldviews is fostered and a strategy to effectively translate mental health knowledge to Aboriginal youth is being implemented, can help this population pursue post-secondary education with tools at their disposal for managing mental health issues (Hallett, 2005; R. Koleszar-Green personal communication, April 15, 2012).

\section{Mental Health among First Nations, Inuit and Métis Youth}

It is difficult to accurately estimate rates of mental health issues among First Nations, Inuit and Métis youth, as they will vary from community to community. It should be noted that the contexts discussed above should always be considered when discussing mental health issues among Aboriginal populations. From a social constructionist lens, it is important to examine culturally-specific constructions of concepts from various social groups as they are likely to be different based on their social and historical contexts (Burr, 2003). Nonetheless, below is a snapshot of different mental health issues that tend to affect Aboriginal youth at a higher rate than the non-Aboriginal population.

Anxiety and depression. Due to the amount of trauma and social adversities experienced by First Nations, Inuit and Métis youth, many experience symptoms of anxiety and depression. The results of the First Nations Regional Health Survey found that $33.8 \%$ of female and 17.2\% of male First Nations youth reported feeling sad, blue or depressed for at least two consecutive weeks at some point in the past 12 months (The First Nations Information Governance Centre, 2012). Bigfoot and Schmidt (2010) discussed the impact of family violence and child victimization on mental health and how such exposure contributes to high rates of posttraumatic stress disorder (PTSD) among American Indian and Alaskan Native populations. More specifically, the prevalence of PTSD among the American Indian and Alaskan Native population 
is $22 \%$, compared to $8 \%$ in the non-Aboriginal population in the United States (Kessler, Sonnega, Bromet, Hughes, \& Nelson, 1995). Although this citation is not in reference to Aboriginal populations in Canada, it provides insight regarding the impact of trauma on the development of mental illnesses. As well, Sack, Beiser, Baker-Brown, and Redshirt (1994) found that, among the $43 \%$ of their American Indian adolescent sample who were diagnosed with a DSM-III-R disorder, anxiety was found to be among the most frequently diagnosed disorder, with $17.4 \%$ presenting with an anxiety disorder. Affective disorders, including depression were also found to be among the highest frequency of diagnosed disorders (i.e., 9.3\%).

Suicide. Suicide rates among First Nations, Inuit and Métis youth have been reported to be five times higher than rates for non-Aboriginal youth (Health Canada, 2003). It should be noted, however, that suicide rates vary substantially from community to community, depending on cultural continuity (Chandler \& Lalonde, 2008). For example, Inuit in Nunavut had a suicide rate of 10 times the rate of the non-Aboriginal population across all age groups from 1999-2003 (Nunavut Bureau of Statistics, 2003). Suicide among Inuit has been identified as a major public health concern in urgent need of attention (Kral, 2009). However, for the Cree Nation in Quebec, rates of suicide have been found to be no greater than the rest of the province's population (Petawabano, Gourdeau, Jourdain, Palliser-Tulugak, \& Cossette, 1994). Differences in suicide rates among First Nations, Inuit and Métis communities provide insight regarding community level factors and cultural continuity, as they can be protective factors against high rates of suicide (Chandler \& Lalonde, 2008; Hallett, 2005; Kirmayer, 1994). Moreover, suicide rates have been linked to the development of other psychiatric disorders, including substance use disorders and symptoms of depression (Kirmayer, 1994). 
Substance use disorder. Results from the First Nations Regional Health Survey, which examined First Nations youth in Canada, found that the prevalence of abstinence from alcohol use is higher among First Nations youth than the non-Aboriginal population of youth (i.e., $61 \%$ versus 47\%, respectively; The First Nations Information Governance Centre, 2012). However, of the First Nations youth who consume alcohol, 56\% reported frequent binge drinking, compared to $39 \%$ of the non-Aboriginal population of youth (The First Nations Information Governance Centre, 2012). One in 10 First Nations youth in Canada were found to smoke marijuana daily or almost daily (The First Nations Information Governance Centre, 2012). Sack et al. (1994) examined American Indian children and adolescents, ages 11 to 18 and found that $43 \%$ of the sample was diagnosed with a DSM-III-R disorder and substance use disorder was found to be among the most frequently diagnosed mental disorder. This study showed that $18.4 \%$ of American Indian adolescents were diagnosed with some form of substance use disorder, with $9.2 \%$ of them being alcohol dependent. Higher rates of solvent abuse among adolescents have been reported for some First Nations and Inuit communities in Canada, in comparison to the nonAboriginal population (Manitoba Office of the Children's Advocate, 2003). Solvent abuse has been linked to loss of self-respect, family breakdown and other adverse social determinants of health (e.g., poverty, unemployment, and a lack of social and economic structures; Dell et al., 2005), which can further influence the development of mental health issues among Aboriginal youth.

It is rare for the prevalence of mental health problems among First Nations, Inuit and Métis youth to be quantified because these populations tend to be difficult to engage in research due to the traumatizing nature of research on Aboriginal people, historically (Battiste, 2000). Although, when this is attempted, it is often done from a positivist Western lens and Aboriginal 
worldviews on mental health and wellbeing are rarely considered. Therefore, the rates of mental health issues stated above should be taken with caution, as Aboriginal worldviews need to be taken into account in order to have an accurate understanding of the prevalence rates.

\section{Aboriginal Perspectives on Mental Health}

The above section provides some insight regarding rates of mental health issues among First Nations, Inuit and Métis youth. However, it is often not recognized that there are differing perspectives of mental health within Aboriginal communities in comparison to mainstream conceptualizations of mental health and it is argued that mental health is itself a social construction, as its definition will vary depending on the social group in question. Therefore, rates reported above should be treated with caution because it is unclear from the studies cited above how mental health was conceptualized (e.g., from an Aboriginal or mainstream worldview).

A worldview refers to the way one interprets the world, including cognitive, perceptual and affective maps that people use to make sense of their interactions with their world, and such interpretations are often manifested through one's culture (Little Bear, 2000). Therefore, many Aboriginal people have differing worldviews from non-Aboriginal people because they are from different cultures. Mental health is often viewed wholistically in a First Nations context, which means that there is emphasis on mental, emotional, physical and spiritual health (Smye, 2001). More specifically, many First Nations people believe that "illness" is the lack of balance or harmony in physical, mental, emotional and/or spiritual components of life (Smye, 2001). In other words, the whole person is the focus when it comes to mental health (Smye, 2001). Wholism refers to "awareness of and sensitivity to the interconnectedness of all things: of people and nature; of people, their kin and communities; and within each person, the interconnectedness 
of body, mind, heart and spirit” (Aboriginal Healing Foundation, 2005, pg. 23). There is a scarcity of literature that specifically addresses Métis perspectives of mental health; however, drawing from the health literature, health perspectives from Métis culture centre around wholism as well (Edge \& McCallum, n.d.). It has been reported that Inuit do not have a term for mental health or mental disorder in their traditional language of Inuktitut (Kirmayer, Fletcher, Corin, \& Boothroyd, 1997). In traditional Inuit culture, mental health was viewed as accidental, a result of breaking some social norm, and/or the intrusion of evil spirits (Kirmayer et al., 1997). Illnesses were often attributed to the interaction between an individual and the spirit of a person, place or thing (Kirmayer et al., 1997). More recently, mental health and wellbeing has been described from an Inuit worldview as being maintained through connectedness with family, community, and the environment (National Aboriginal Healing Organization, 2009).

Many Aboriginal communities focus on general wellbeing, rather than specific symptoms (Alberta Mental Health Board, 2006; Blackstock, 2008). Wellness has also been described as a positive state of wellbeing and strength evident in individuals, families and communities as well as the relations among them (Canadian Population Health Initiative, 2009). The National Aboriginal Community Controlled Health Organization (NACCHO) stated that mental health should be considered within a broad context of health and wellbeing (NACCHO, 1993). More specifically, mental health issues should be recognized as encompassing oppression, environmental factors, economic factors, stress, trauma, grief, and cultural genocide, in addition to psychological processes (NACCHO, 1993).

Cain and colleagues (2011) examined the mental health perspectives of First Nations, Inuit and Métis people in Canada who were HIV positive and who experienced depression. Many participants in their sample did not describe their depression in psychological terms such 
as feeling hopeless or sad, rather they experienced depression relationally and as a disconnection from other people, communities and culture. Since this perspective is different from Western ways of defining depression, participants found it difficult to recognize and label their feelings as “depression" (Cain et al., 2011). Such differing perspectives on mental health may make it difficult for service providers and institutional organizations to recognize and help those who have culturally specific experiences of depression and other mental health issues (Cain et al., 2011). Depression and other mental health issues have been described by Aboriginal people from Australia as characteristic of the person (i.e., part of who that person is) and are only pathological when the issue becomes visible to others, such as crying or engaging in high-risk behaviours (Vicary \& Westerman, 2004). It is unclear whether this perspective can be applied to Aboriginal Nations in Canada, but further highlights the mental health concept from an Aboriginal worldview as being different from a positivist Western worldview.

Aboriginal worldviews on mental health have been examined for First Nations youth from Northern Manitoba (Isaak \& Marchessault, 2008). Most First Nations youth included in the study spoke about the four dimensions of the medicine wheel in reference to health, in general. A medicine wheel is a wholistic model that can be applied to various phenomena. With regard mental health, specifically, First Nations youth spoke about the importance of making good choices and the necessity of being educated about positive lifestyle options, so that one can make good choices. First Nations youth spoke about how behaviours and choices were often influenced by their peers, parental support, or culture (Isaak \& Marchessault, 2008). This study did not discuss conceptualizations of mental health for Inuit and Métis youth.

Aboriginal perspectives on mental health have been examined with Aboriginal adults from various locations in Canada and Australia as cited above, yet there is little research on 
mental health perspectives of Aboriginal youth. Mental health perspectives have not been examined with Aboriginal students, specifically, who may be in their youth, or with Aboriginal people who are in an academic context, both of which were examined in the present study.

Although there is a lack of clarity regarding how mental health can be defined from an Aboriginal worldview and among Aboriginal students, Aboriginal community members often agree that mental health issues are a problem in their communities and that, when they are addressed, this can contribute to educational achievement and, therefore, eventual selfdetermination within their communities (e.g., R. Koleszar-Green personal communication, April $15,2012)$

\section{Education as a Way Forward}

Paradoxically, even though the present education system continues to traumatize many First Nations, Inuit and Métis children and youth, and education can be considered to be a social adversity for many as cited above, it has been proposed that this same system can help move Aboriginal communities forward. More specifically, it has been suggested that, when a culturally safe space is available, the completion of post-secondary education for First Nations, Inuit and Métis populations is not only beneficial for their communities, but also beneficial for the Canadian society at large. The term "education is our buffalo" has been used by First Nations Elders to signify their belief that, if any positive changes are to be made in their communities, it will be made through educational attainment (Alberta Education, 2002). The birth of a white buffalo is considered to be sacred in many Aboriginal communities and represents hope, rebirth, and unity for Nations that depended on the buffalo for survival (Alberta Education, 2002). There is the belief among Aboriginal communities that education can foster hope, rebirth and unity among Aboriginal students (Alberta Teachers Association, 2006) and that education will give 
Aboriginal youth the chance to succeed (Alberta Education, 2002). Not only will educational attainment improve social conditions for Aboriginal Peoples, such as employment and housing, but it will also contribute to skilled workers in Canadian society, which will contribute to Canada's economic growth and sustainability (Brunnen, 2004). It is argued that through addressing barriers to educational achievement, such as mental health issues, Aboriginal students will be more likely to complete their post-secondary education and successfully contribute to their communities. One way to address mental health barriers to educational achievement is to provide Aboriginal students with mental health knowledge as well as internal and external resources to cope with mental health issues through a knowledge translation strategy.

\section{Knowledge Translation in an Aboriginal Context}

Knowledge translation (KT) is defined differently depending on the context of focus. More specifically, in Aboriginal contexts, KT has been defined as sharing what is known about living a good life (Kaplan-Myrth \& Smylie, 2006). KT has also been described as encompassing many processes, which include, but are not limited to sitting together at the kitchen table sharing knowledge, translating Western language into community language, involving community members in shaping research, and putting research into practice (Kaplan-Myrth \& Smylie, 2006).

$\mathrm{KT}$ is often described in reference to the gap between research and practice. The Two Communities theory posits that this gap stems from stakeholders' differing definitions of evidence (Caplan, 1979). Researchers (i.e., community \#1) define knowledge as developed using a systematic and scientific process (Caplan, 1979). Individuals involved in clinical practice (e.g., frontline service providers, managers, and policy makers; community \#2) have broader definitions of knowledge, which includes locally relevant information, experience, and culturally-based information, in addition to scientific research (Jack, Brooks, Frugal, \& Dobbins, 
2010; Smylie et al., 2003). The research-practice gap exists in Aboriginal contexts; however, knowledge is defined more broadly than "research". Aboriginal Peoples have been applying their own science in the form of oral traditions, experiential knowledge, and cross-cultural sharing for centuries (Kaplan-Myrth \& Smylie, 2006). Understanding the many different sources of knowledge that are important to Aboriginal people is key in filling the gap between knowledge and the application of such knowledge in Aboriginal communities (Canadian Institutes of Health Research, 2009).

Due to a rich history of KT in Aboriginal contexts and the high prevalence of mental health issues among First Nations, Inuit and Métis populations, the use of KT to improve the wellbeing of Aboriginal people is very appropriate (Canadian Institutes for Health Research, 2009). Using KT to improve Aboriginal wellbeing is not entirely new, as Aboriginal Peoples have believed for generations that the whole purpose of knowledge generation is to use it to increase the wellbeing of their community members (Canadian Institutes for Health Research, 2009). It is recommended that $\mathrm{KT}$ in an Aboriginal context combine both Aboriginal and nonAboriginal conceptualization of KT, as there is value in both ways of knowing (Canadian Institutes of Health Research, 2009). Therefore, the present study will use the Aboriginal definition of KT and combine Western and Aboriginal theories of KT to guide its process.

Most research on KT within an Aboriginal context has been done in the healthcare field, rather than the mental health field. Ranford and Warry (2006) conducted a survey with Aboriginal healthcare researchers working in Ontario and identified two KT strategies to be effective in closing the research-practice gap in the healthcare field: oral presentations to health professionals and oral presentations to frontline workers. These two KT strategies stemmed from 
an extensive list including videos, website publications, storytelling, dramatic plays, and policy briefs.

Important KT lessons have been learned from implementing a diabetes prevention program in Kahnawake Schools (which is in an Aboriginal community; MacCaulay, Paradis, Potvin, \& Cross, 1997). Avenues for dissemination were the local newspaper, local radio stations, television programs and common bulletin boards (MacCaulay et al., 1997). Newsletters were sent home with parents with updates on the outcomes of the program and parents had the opportunity to be involved in the program during report card and orientation days. Lessons learned about potentially effective KT strategies included translating locally based and locally relevant knowledge to the people and community, contextualizing information so it makes sense, using appropriate community language, actively (rather than passively) presenting information to people, and having the community members translate messages to the community (MacCaulay et al., 1997).

In a study by McShane, Smylie, Hastings, and Martin (2006), they conducted a series of interviews and focus groups with urban Inuit from Ontario to gather information on their preferences for receiving health information. Learning visually, including community Elders, including cultural interpreters, and having community cohesion were important in the process of sharing health information (McShane et al., 2006). Major sources of health information were found to be family members and sources within the Inuit community (McShane et al., 2006). The most effective KT strategy was found to be direct communication, through individual or group interactions. A KT tool was developed based on this information, which involved a CD-ROM multimedia health information tool and was found to be an effective tool to transmit health information to the Inuit community (McShane et al., 2006). Conclusions from this study suggest 
that effective KT strategies may differ significantly in Aboriginal contexts in comparison to mainstream contexts (McShane et al., 2006).

In addition to Inuit, preferences for receiving health information among First Nations and Métis populations have been examined. Smylie et al. (2009) conducted a study with semi-urban First Nations and urban Métis communities in Ontario and found distinct differences in KT preferences between such communities. Regarding the semi-urban First Nations community, participants emphasized either traditional health knowledge or biomedicine, depending on their connection to their First Nations traditions (Smylie et al., 2009). They also preferred individual communication (rather than group communication) of health information due to concern about privacy and stigma as related to certain health issues (i.e., addiction; Smylie et al., 2009). Finally, regarding the Métis community included in this study, participants emphasized a lack of community cohesion as an obstacle to getting health information from community members. They also discussed difficulty relating to their Métis identity due to the shame associated with being Métis that has been passed down generationally, which affected the value they placed on Métis-specific knowledge (Smylie et al., 2009). The common themes among all three Aboriginal groups that have been found to impact the KT process included the value of experiential knowledge, the influence of community cohesion on dissemination, a preference for health messages generated within the community, disseminating information through family and community networks, and considering the impact of colonization (e.g., how there are differing levels of tension that may exist between Indigenous and positivist Western knowledge systems; McShane et al., 2006; Smylie et al., 2009).

In addition to the examples outlined above, Hanson and Smylie (2006) outlined a KT policy that can be used when working with Aboriginal communities. This policy states that the 
KT process should ensure that information generation and translation will benefit the community and that inherent diversity within and between Aboriginal communities should be recognized when working with knowledge. The policy states that KT must protect individual and community identity as being "Indigenous" and speak to how both Aboriginal and non-Aboriginal knowledge can enhance Aboriginal identity. The policy also outlines that a KT strategy must incorporate one or all categories of knowledge (including old and new knowledge), use Aboriginal languages when appropriate, use wholistic means of knowledge sharing, and share knowledge honestly and generously. Hanson and Smylie (2006) suggest that knowledge can only be effectively translated when relationships have been built on respect and trust and when the KT project is relevant to community needs. The authors state that the information being translated may need to go back and forth between community members before it is accepted and that mechanisms need to be put into place to ensure that the Aboriginal knowledge to be translated will be protected. Finally, the KT practice should emphasize dialogue for active participation from community members and create a culturally safe space for the KT activity (Hanson \& Smylie, 2006). These recommendations will be considered while developing the Knowledge Translation Phase of the study.

KT has been defined differently in Western contexts in comparison to Aboriginal contexts. According to the Canadian Institutes of Health Research (CIHR), knowledge translation (KT) is a process that involves synthesis, dissemination, exchange and application of knowledge to improve health (Canadian Institutes of Health Research, 2009). In contrast to the KT examples provided above, positivist Western strategies of KT have a stronger focus on translating research into practice and often do not take Aboriginal culture or knowledge systems into consideration. Therefore, Western KT strategies are often not applicable translating 
knowledge to Aboriginal community members, which the present study aims to do. However, through briefly examining Western KT strategies that have been implemented, they may provide insight into possible ways to translate mental health information, which could be adapted for use within Aboriginal contexts. Moreover, as cited above, it is recommended that both Aboriginal and Western worldviews be considered when using KT in an Aboriginal context due to the value in both ways of knowing (Canadian Institutes of Health Research, 2009).

Educational strategies have been identified as a commonly used KT strategy within the healthcare field and includes distributing educational materials to professionals; however, most studies examining these interventions have concluded that its effectiveness is limited (Freemantle et al., 1995). It has been found that Aboriginal patients' experiences of communication with their physician depend on the acknowledgement of their histories as Aboriginal people, how much they trust their physician, and how much time they are given during the medical interview (Towle, Godolphin, \& Alexander, 2006). More specifically, it was found that when sufficient time is allotted for the medical interview, history became less of a barrier to healthcare for Aboriginal people and communication and trust was able to grow, making it more likely that Aboriginal patients understood the information provided by the physician, which increased desired health outcomes (Towle et al., 2006). In particular, Aboriginal patients' were able to use their voices and felt like there was time for their voices to be heard by their physicians (Towle et al., 2006). Using computerized reminders have been found to be effective in the healthcare field, especially with regards to prevention measures (e.g., vaccinations and cancer screening; Grimshaw, Eccles, Walker, \& Thomas, 2002). Multiprofessional collaboration, where professionals from various backgrounds collaborate with one another to increase research use, has been shown to have mixed findings (Grol \& Grimshaw, 
2003). Mass media campaigns are another frequently used KT intervention, which has been found to be effective across various studies within the healthcare field (Grilli, Freemantle, Minozzi, Domenighetti, \& Finer, 2000). All of these options were considered when designing the KT activity as well as barriers to uptake, setting, target group preferences and the state of the information (Grol \& Grimshaw, 2003).

Barwick and colleagues (2005) provides tips for developing strategies to engage knowledge users in KT strategies and some of which overlap with recommendations previously reviewed for use in an Aboriginal context (e.g., Smylie et al., 2009). Such tips include active collaboration and partnership with participants, face-to-face translation of knowledge, understanding knowledge users' attitudes toward the knowledge given, and providing access to the knowledge (Barwick et al., 2005).

In addition to the various strategies that have been empirically examined and tips provided by researchers in the KT field (e.g., Barwick et al., 2005), there are many models used to implement KT. The "knowledge to action" model has been commonly used to represent the process of KT (Graham et al., 2006). The knowledge to action process has two phases: knowledge creation and action (Graham et al., 2006). During knowledge creation, created knowledge becomes increasingly distilled and refined (Graham et al., 2006). As knowledge is refined, it becomes more useful to knowledge users. Once knowledge is tailored to the activities of knowledge users, the action phase begins. During the action phase knowledge is applied, which involves identifying problems or issues that deserve attention, so that knowledge can be adapted to the appropriate context, barriers to knowledge uptake can be assessed and interventions can be tailored to such barriers. Once interventions for knowledge uptake are 
tailored, knowledge use is monitored, the impact of knowledge uptake is determined and knowledge use can be sustained (Graham et al., 2006).

Although ways to effectively translate knowledge in Aboriginal and Western contexts have been examined, the healthcare field has dominated such examination and little attention has been paid to the mental health field or to community members as knowledge users. Work examining KT strategies in an Aboriginal context has mostly been done with Aboriginal adults and, to the principal investigator's (CJD) knowledge there is yet to be a study examining KT in an Aboriginal context with youth and/or students. Therefore, the present study aimed to address this gap.

\section{Community-Based Research}

CIHR's Guidelines for Health Research Involving Aboriginal People (Canadian Institutes for Health Research, 2011) and Chapter 9 in the Tricouncil Policy Statement: Research Involving First Nations, Inuit and Métis peoples of Canada (Government of Canada, 2012) recommends using a participatory action approach when conducting research with Aboriginal people. Due to the nature of a dissertation, which is to increase autonomy and independence among the student researcher, community-based research and its tenets were emphasized over a participatory approach to research. Community-based research is an approach that emphasizes collaboration, participation and social justice over positivist assumptions of objectivity (Hall, 1993).

Community-based research involves community members in the research process and assumes that such involvement makes research more understandable, responsive and pertinent to these communities (Israel, Schulz, Parker, Becker, 1998). As well, it has been noted that communitybased research can result in empowerment among community members, which may allow individuals to make individual and social changes (Wallerstein \& Duran, 2003). The primary aim 
of community-based research is to improve health and decrease health inequalities through collaborating with, and involving the communities who, in turn, take actions to improve their own health (Baum, MacDougall, \& Smith, 2006). Community-based research differs from traditional research as it aims to produce change and improvement through action (Minkler, 2000; Reilly, Doyle, Bretherton, \& Rowley, 2008). Such action toward change is pursued through a reflective cyclical process of data collection, such that data are collected, there is reflection or discussion of data between the researchers and community, action is implemented based on such reflection, and the cycle repeats (Cornwall \& Jewkes, 2010; Israel et al., 2001; Reilly et al., 2008). Community-based research provides an agenda of how those being studied will be involved in the research process and individuals are considered regarding all aspects of the project including how they will be affected by the research and plans for collaboration throughout the entire process (Israel et al., 2001; Kelly, 2005; Reilly et al., 2008).

Community-based research promotes balance in relationships and power between academic researchers, partner organizations, community participants and decision-makers (Chouinard \& Cousins, 2007; Evans, Hole, Berg, Hutchinson, \& Sookraj, 2009). Implementing community-based research differs from past studies in which research was done on First Nations, Inuit and Métis people without their consent and without considering community needs (Battiste, 2000; Humphrey, 2001; Reilly, et al., 2008). Using a collaborative approach through community-based research facilitates a trusting relationship between researchers and First Nations, Inuit and Métis people, so that society can benefit from the valuable insights of Aboriginal community members. Community-based research also builds capacity in these communities so members can take action to improve their circumstances (Cornwall \& Jewkes, 2010; Flicker, Savan, Kolenda, \& Mildenberger, 2008), which facilitates self-determination: a 
goal of many Aboriginal communities (Asch, 2002). Collaboration exists from the initiation of the research project to the dissemination of the research findings (Israel et al., 2001; Reilly et al., 2008).

Community-based research is in line with the Ownership, Control, Access, and Possession principles (OCAP). OCAP has been referred to as a "political response to colonization and knowledge production in reproducing colonial relations" (Schnarch \& First Nations Centre, \& National Aboriginal Health Organization, 2004). "Ownership" refers to the relationship of the First Nations community to its cultural data and states that the community should own such data. "Control" refers to an extension of the right of First Nations Peoples to maintain control over all aspects of their lives, to research and the ability of First Nations people to assert their right to control data and information that might impact them (Schnarch \& First Nations Centre, \& National Aboriginal Health Organization, 2004). Such control extends from the conception to completion of a research project, which is very much in line with principles of community-based research. "Access" refers to how First Nations Peoples must have access to data on themselves and communities, regardless of where the data is held. "Possession" refers to a mechanism through which ownership is accomplished. OCAP is considered to be an active concept that opens up avenues for self-determination in the areas of research, and provides hope for positive changes within First Nations communities (Schnarch \& First Nations Centre, \& National Aboriginal Health Organization, 2004). Even though the OCAP principles refer to First Nations populations, specifically, these principles have been applied to KT in Aboriginal contexts more generally (Kaplan-Myrth \& Smylie, 2006) and, therefore, have been applied to the present study, which involved First Nations, Inuit and Métis participants. This study was developed in partnership with Ryerson Aboriginal Student Services (RASS) in Toronto, ON and 
is consistent with their goals and future directions. As well, in addition to this dissertation, upon its successful defence, a community report will be developed for the partnering organizations to access and use as necessary.

\section{Relationship with Community Partner}

This project was completed in partnership with Ryerson Aboriginal Student Services (RASS). RASS is a community organization located at Ryerson University in Toronto, ON and "provides a culturally supportive environment to promote academic excellence and serves as a place to balance academic learning with traditional teachings and culture" (Aboriginal Services, 2014). RASS provides service to First Nations, Inuit and Métis students (Aboriginal Services, 2014). The principal investigator (CJD) has had a relationship with RASS since 2009. In 2011, she approached the academic advisor about whether there might be any opportunity to work with RASS during the course of her dissertation. The academic advisor suggested that the principal investigator (CJD) implement something educational around mental health for Aboriginal students at Ryerson. The director of RASS was also called into the meeting and agreed that this idea would fit with the needs of RASS and she provided some suggestion regarding how to collect the data. Upon commencement of this meeting, the principal investigator (CJD) spent a few months consulting with her supervisors and fine-tuning the idea, she then met with the academic advisor again to discuss the evolution of the original idea and ensure that it was still in line with the needs of the community organization. The academic advisor also read the principal investigator's (CJD) dissertation proposal and provided feedback. Time was spent building relationships with Elders at RASS by scheduling meetings with them to discuss the project and ensuring that they were in support of the project. A letter of support and research agreement was drafted and signed by the academic advisor and the principal investigator (CJD). The academic 
advisor, as well as other staff and community members at RASS were involved throughout the project in various ways, including research question development, recruitment, data analysis, and data interpretation.

\section{Epistemological Lens}

Tool development. The main epistemological lens guiding the research in phase one was social constructionism. Social constructionism differs from positivist psychology in a few key ways. First, social constructionism rejects the assumption that there are essences inside things or people that make them what they are (Burr, 2003). Social constructionism does not assume that knowledge is a direct perception of reality and is, therefore, skeptical of positivist claims that are characteristic of much of the work that mainstream psychology contributes (Burr, 2003). Social constructionism criticizes mainstream psychology for adopting imperialism and colonialism in which positivist Western ways of knowing are assumed to be the "right" way, as it is argued that theories of psychology are time and culture-bound (Burr, 2003). Most of mainstream psychology assumes that language is an expression of thought, rather than a precondition of thought, as is assumed by social constructionism. More specifically, it is assumed by social constructionism that the concepts and categories that provide meaning in people's lives are created by language. Therefore, according to social constructionism, language is necessary for thought and meaning, rather than language being seen as a passive vehicle for thought and emotion as is often the case in mainstream psychology (Burr, 2003). According to social constructionism, language is a form of action, since as language is used; ideas, experiences, beliefs and concepts continue to be constructed (Burr, 2003). Moreover, instead of looking for explanations for social phenomena inside the person (e.g., attitudes and cognitions) as does mainstream psychology, social constructionism focuses on the social practice itself to understand phenomena. Social 
constructionism emphasizes processes, rather than structures (e.g., personality traits and models of memory). Therefore, social constructionism is concerned with how forms of knowledge are achieved by people through social institutions and social products, and knowledge is seen as something that people do together (Burr, 2003).

Four main tenets define social constructionism. First, social constructionism is critical of the idea that conventional knowledge is objective and unbiased and is in opposition to positivism and traditional science, as categories that human beings use on a daily basis do not necessarily represent real divisions (Burr, 2003; Gergen, 1985; Gergen, 2009; Nightingale \& Cromby, 2002). For example, in psychology, mental disorders are often defined using a Diagnostic Statistical Manual (DSM), which categorizes various mental disorders by symptoms (e.g., American Psychiatric Association, 2000). Mental health is therefore defined as one being devoid of any one of the disorders outlined by the DSM criteria in mainstream contexts. However, this is not necessarily the reality of mental health for all, as it may vary depending on the social group of interest. For example, various groups of Aboriginal people in Canada often have different constructions of mental health as outlined above. Knowledge translation is defined by the Canadian Institutes of Health Research (CIHR) as outlined above, but is defined by people in Aboriginal contexts in a different way, which suggests that CIHR's definition does not necessarily represent objective reality, but is only one possible way of conceptualizing it.

Second, the ways in which we understand the world is historically and culturally specific (Burr, 2003; Gergen, 1985; Gergen, 2009). Knowledge is a product of history and culture and, therefore, since Aboriginal worldviews, culture, and history are often different from mainstream worldviews, culture, and history, it is logical to suggest that Aboriginal knowledge will vary and be specific to particular Nations. This tenet of social constructionism states that one should never 
assume that the mainstream way of knowing is better, in terms of being closest to the objective truth, than other ways of knowing, which recognizes and validates knowledge that comes from other cultural groups (Burr, 2003; Gergen, 1985). Conceptualizations of mental health and knowledge translation are specific to Aboriginal peoples and grounded in their historical experiences and cultures. For example many First Nations have traditionally believed in wholistic healing and interrelatedness, which is reflected in their beliefs about mental health (i.e., that it is more about wellbeing and achieving balance than symptoms-focused; Smye, 2001). As well, such wholism and interrelatedness is reflected in conceptualizations of knowledge translation, as it is often thought of as sharing what is known about living a good life to all community members, as we are all connected and, therefore, interrelated (CIHR, 2009).

Third, social processes sustain knowledge (Burr, 2003; Nightingale \& Cromby, 2002). This tenet assumes that knowledge is not derived from nature, rather, people construct such knowledge through interactions with each other and, from a social constructionist lens, language is central to such social interactions (Burr, 2003; Gergen, 2009). Interactions between people are seen as the process through which shared versions of knowledge are constructed (Burr, 2003; Gergen, 2009). For example, Aboriginal people have shared meanings of wellbeing and knowledge translation that are often different from mainstream understandings and, since language is such a key part of many Aboriginal cultures, conceptual meaning for many Aboriginal groups is often developed through oral communication, which further supports the application of social constructionism to the present study (i.e., phases one and three, in particular).

The fourth central tenet of social constructionism states that knowledge and social actions go together (Burr, 2003; Gergen, 2009; Potter \& Wetherell, 1987). This tenet refers to numerous 
possible social constructions of the world, but each construction will invite a different kind of action from people to sustain such knowledge (Burr, 2003). For example, the Aboriginal conceptualization of mental health as being wholistic is reflected in various services that have been designed around this perspective. More specifically, the Aboriginal Alcohol and Drug Worker program offered through Friendship Centres in Ontario focuses on the wholistic needs of their clients (Ontario Federation of Indigenous Friendship Centres, 2013). As well, knowledge translation being conceptualized as sharing what is known about living a good life has been put into action through published papers that reference this definition (e.g., CIHR, 2009).

Research from a social constructionist lens is often conducted qualitatively, since this is ideal for gathering data; however, quantitative analysis can be used as well (Burr, 2003). The Tool Development Phase will use primarily qualitative data collection and analysis. There are also some methodological issues that need to be considered. First, objectivity is viewed as impossible when using social constructionism, since reality is being constructed and, therefore, the researcher must recognize that research is a co-production (Burr, 2003). A final methodological issue that will be reviewed is reliability and validity. Since social constructionism states that there is no objective reality, reliability and validity, as they are understood in positivist mainstream psychology, are not applicable to work using the social constructionism lens (Burr, 2003). To enhance the rigour of social constructionist research, Taylor (2001) has recommended that one provide in-depth information about the steps in the analytic procedure. Such methodological issues and recommendations have been considered and adopted in the present study. In using social constructionism as the epistemological lens through which phases one and three of this study was completed means that it was assumed that everything examined during these phases were constructed by Aboriginal community members. 
As well, the concepts examined in the present study (i.e., mental health and knowledge translation) were grounded in Aboriginal community members' cultural and historical contexts. Most data were collected orally, which is directly in line with social constructionism. More specifically, the first study phase aimed to explore Aboriginal understandings of mental health and effective ways to translate mental health knowledge to Aboriginal students. Responses were collected qualitatively, using focus groups and allowed participants to develop a concept(s) of mental health, understand gaps in knowledge about mental health and develop a knowledge translation activity to communicate mental health knowledge to Aboriginal students.

Knowledge translation phase. Phase two of the present study aimed to implement and evaluate a KT activity that was developed from the first phase. Due to the evaluation component of this phase, the epistemological lens used to guide this phase was critical realism, while still adhering to a community-based approach. Critical realism assumes that reality exists, but only imperfectly and is probabilistically apprehensible (Archer, Bhasker, Collier, Lawson, Norrie, 1998; Danermark, Ekstrom, Jakobson, \& Karlsson, 2002; Collier, 1994; Denzin \& Lincoln, 2005). It assumes that findings are probably true and hold that knowledge about the world is socially constructed (Denzin \& Lincoln, 2005). This lens is fitting with the present phase because its objective was to understand how helpful the knowledge translation activity was for Aboriginal students and, therefore, it was assumed that their feedback reflected the reality of their experiences of the activity. However, through conducting a qualitative evaluation of this feedback and working to contextualize the results of this phase, it is recognized that such reality is in fact imperfect and is likely impacted by one's social and historical context. Critical realism goes beyond statements of regularity to examine mechanisms, process and structures that account for patterns that are observed (Denzin \& Lincoln, 2005). Although the objective of this 
phase was not to examine mechanisms through which the KT activity was helpful, it did aim to contextualize the history as well as current systems that may account for the findings within this phase. Within the critical realism lens, traditional evaluation criteria (e.g., internal validity) are emphasized, yet it rejects methodological individualism and universal claims to truth (Denzin \& Lincoln, 2005).

Community follow-up/debriefing phase. The third study phase aimed to follow-up with and debrief key informants that had been involved with the project regarding their impressions of how the project went, ways the project addressed community needs, and ideas for the future of mental health. This phase of the project was guided by a social constructionist lens, as is defined above. It addition to the tenets and methodological considerations outlined above, social constructionism aims to create a democratization of the research relationship, where research is conducted collaboratively and informed by the needs and aims of the participants. More specifically, Orford (1992) recommends using social constructionism with community psychology because community psychology aims to empower participants, especially when using community-based research, which compliments the aims of social constructionism and is very much in line with the goals of this study phase. Democratization relates to a term that is often used in reference to social constructionism, called reflexivity, as this refers to equal status between the researcher and the participants (Burr, 2003). Empowerment of participants and aspirations of moving toward equal status between the participants and the researcher (while recognizing the privilege associated with being an academic researcher) was exactly the aim of the entire study and phase three taps into this goal (reflexivity has also been defined as an account being a description of an event as well as part of the event due to the constitutive nature 
of language; Burr, 2003). This phase emphasized the research process as is in line with social constructionism.

This phase assumes that 'research' is not objectively defined, but rather it is time and culture-bound. Specifically, research and the process of research is defined differently depending on one's social context. Within mainstream psychology, research is often for personal and professional interests; methodologies are chosen for disciplinary conventions, 'objectivity' and 'truth'; the researcher conducts data collection, interpretation and analysis; and outcomes are emphasized (Cornwall \& Jewkes, 1995). Within community psychology and for research conducted with Aboriginal people, in particular, research is done for local people, methodologies are chosen for empowerment of communities and mutual learning among the researcher and the community; local people are involved in the data analysis, interpretation and analysis; and the research process is emphasized (Cornwall \& Jewkes, 1995; Government of Canada, 2012).

Within an Aboriginal context, in particular, there is often an expectation that the researcher form relationships within the community and that culturally safe research methods are implemented, including traditional ceremonies (Baskin, 2005). These assumption as well as the assumptions outlined above with regard to social constructionism as applied to phase one are in line with the aims of the Follow-Up/Debriefing Phase of the current project and, therefore, questions were developed, and data were collected, analyzed, interpreted and written from this lens.

\section{Thematic Analysis}

Social constructionism lends itself well to thematic analysis to analyze the qualitative data collected (Braun \& Clarke, 2006). Thematic analysis can also be used when conducting research from an essentialist or realist lens; however, experiences and meaning from participants are reported as reality when using these lenses, which is not the case when using social 
constructionism. In contrast, thematic analysis from a social constructionist lens identifies patterns of experiences as being socially produced, rather than inhering within individuals (Burr, 1995) and report latent themes, which require interpretive work (Braun \& Clarke, 2006).

Thematic analysis from a realist lens reports semantic themes, which describe these themes without looking for anything beyond the semantics of experiences (Braun \& Clarke, 2006).

Thematic analysis involves constant moving back and forth between the data set and the extracts of data that have been coded and, therefore, it is described as a recursive, rather than linear process of analysis (Braun \& Clarke, 2006). Writing has also been described as a key part of the analysis process (Braun \& Clarke, 2006). Braun and Clarke (2006) have outlines six guidelines for conducting a thematic analysis being conducted from either a realist or social constructionist lens. The first involves becoming familiar with the data. This can be done either by collecting the data, transcribing the data, or immersing oneself in the data through repeatedly reading the transcripts. One can begin making notes about coding during the process of familiarizing oneself with the data. The second phase focuses on generating initial codes from the data. One will often have a long list of codes to be used in the next phase upon completion of this phase. The third phase involves searching for themes, where one begins to organize the data using the codes that were developed in the previous phase of analysis. Here, it is recommended that one gather all data relevant to each code or theme. The next phase involves reviewing each theme, which requires checking that each piece of data is relevant to each theme. The fifth phase is to define and name themes, which will require ongoing analysis. The final phase involves producing the report, which is the final opportunity for analysis and is also known as the writing phase (Braun \& Clarke, 2006). These guidelines will be used in the context of social constructionism for phase one and three and for evaluation purposes in phase two. 


\section{Present Study}

This study had the following objectives: (1) To understand mental health perspectives from an Aboriginal worldview, gaps in Aboriginal students' mental health knowledge, and the best ways to communicate mental health knowledge to Aboriginal students; (2) to develop and implement a KT strategy, and evaluate its ability to increase Aboriginal students' mental health knowledge and self-efficacy in managing their own mental health and wellbeing issues; and finally, (3) to follow-up on the key emphases of community-based research by assessing the community members' reactions to the research process and ensuring that community needs were met. 


\section{Method}

\section{Overview}

The present study consisted of three phases. Phase one was called the Tool Development Phase during which Aboriginal post-secondary students participated in two focus groups (and one individual interview) and RASS staff members participated in one focus groups (and one individual interview) to gain an understanding of their perspectives on mental health, gaps in students' knowledge of mental health, and the best ways to translate mental health information to Aboriginal students. Phase two was called the Knowledge Translation Phase, which was informed by the Tool Development Phase. During the Knowledge Translation Phase, participants were asked to respond to questions related to mental health facts, resources, and coping strategies before and after the strategy was implemented. They were also asked to provide feedback regarding the effectiveness of the KT strategy. After the KT activity, students participated in an individual interview a month later to determine whether they retained knowledge and put such knowledge into practice throughout their daily lives (i.e., knowledge to action; Grimshaw et al., 2002). The third phase was called the Community Follow-Up/Debriefing Phase. Key informants of the project were interviewed regarding their impressions of the project and whether it met the needs of the partnering community. Each phase was conducted at Ryerson University in Toronto, Ontario.

\section{Participants}

Tool development phase. A sample of nine First Nations, Inuit and Métis postsecondary students were recruited from RASS in Toronto, Ontario to participate in the first phase of the study (i.e., four participated in the first focus group, one participated in an individual interview, and four participated in the second focus group). Participants were required to self- 
identify as Aboriginal, which could include First Nations, Inuit, and Métis, as well as non-status Indian. They were also required to be 18 years or older. In addition to Aboriginal students, four RASS staff members (e.g., Elders, faculty, RASS staff) were recruited from RASS in Toronto, Ontario to participate in the first phase of the study (i.e., three participated in the focus group and one was interviewed individually at a later date). To be included in the study, RASS staff members were required to have worked with Aboriginal students in the past or presently. The recruited RASS staff members were required to self-identify as Aboriginal, which could include First Nations, Inuit, and Métis as well as non-status Indian. Finally, the RASS staff members were required to be 18 years or older.

Knowledge translation phase. A sample of five First Nations, Inuit and Métis postsecondary students and one Elder were recruited from RASS in Toronto, Ontario to participate in providing feedback on the questionnaires developed by the principal investigator (CJD). These questionnaires were used during the knowledge translation activity and, therefore, they were piloted beforehand. Participants who provided feedback on these questionnaires were required to self-identify as Aboriginal, which could include First Nations, Inuit, and Métis as well as nonstatus Indian. Participants could overlap with those recruited for the Tool Development Phase of the study.

Once data from the Tool Development Phase was analyzed and the questionnaires were finalized through feedback gathered, the Knowledge Translation Activity was ready to be implemented. A sample of four First Nations, Inuit and Métis post-secondary students were recruited from RASS in Toronto, Ontario to participate in the implementation of the KT strategy. These participants could be the same as those recruited in the first phase and those recruited to provide feedback on the questionnaires. Participants were required to self-identify as Aboriginal, 
which could include First Nations, Inuit, and Métis as well as non-status Indian. They were also required to be 18 years or older. One Elder was recruited from RASS in Toronto, Ontario to help facilitate the final KT strategy. This Elder was the same as the Elder recruited to provide feedback on the questionnaires and was required to be a past or present employee of RASS. The recruited Elder was required to self-identify as Aboriginal, which could include First Nations, Inuit, and Métis as well as non-status Indian. Finally, the Elder was required to be 18 years or older.

Follow-up/debriefing phase. A sample of five key informants were recruited to provide feedback on the research process and to debrief about how the project addressed their community needs. Key informants were required to be involved in the project at various points in time with varying roles (e.g., recruiting participants, helping to implement the activity, and/or contributing ideas and research questions at the inception of the project). The recruited key informants were required to self-identify as Aboriginal, which could include First Nations, Inuit, and Métis as well as non-status Indian. Finally, key informants were required to be 18 years or older.

\section{Materials}

During all phases, responses were audio-recorded. Audio-recordings were collected using a Roland Ederol R-09HR, which is a high resolution audio recorder. All qualitative data analyses were completed using a qualitative analysis program called NVivo 8.0. Quantitative analyses (i.e., descriptive statistics) were completed using SPSS.

\section{Design}

The present study consisted of three phases. The Tool Development Phase incorporated an exploratory design using open-ended questions and qualitative analysis to examine mental health perspectives, gaps in mental health knowledge and KT preferences. The Knowledge 
Translation Phase incorporated a pre/post and follow-up design, where questions were asked at three different time points: prior to the implementation of the KT activity, immediately upon completion of the KT activity, and one month after the KT activity to examine knowledge uptake as well as knowledge use. The third phase also used an exploratory design, which was similar to the first phase.

\section{Procedure}

Participant recruitment. Participants for the first two phases were recruited through the Aboriginal Academic Support Advisor, the principal investigator (CJD), the Elder on staff at RASS in Toronto, ON, and posting flyers (see Appendix A for Student Recruitment Flyer, and Appendix B for Staff Recruitment Flyer). The RASS Academic Support Advisor, the principal investigator (CJD), and the Elder on staff first identified eligible and interested students and staff members, then described the study to potential participants, and asked them if they wished to participate (see Appendix C for the Student Recruitment Script: Tool Development Phase, Appendix D for the Staff Recruitment Script: Tool Development Phase, and Appendix E for Student Recruitment Script: Knowledge Translation Phase). If students and RASS staff members were interested in participating, the Academic Support Advisor, Elder, or principal investigator (CJD) informed them of when the student focus group and RASS staff member focus group (for the Tool Development Phase) or KT activity (for the Knowledge Translation Phase) would take place. Students were recruited to provide feedback on questionnaires to be used during the Knowledge Translation Phase by the principal investigator (CJD) through email or through simply asking them for their time when the principal investigator (CJD) and students were around the RASS student space together. Elders were recruited from RASS by the principal investigator (CJD) and were presented with tobacco upon requesting their involvement in the 
Tool Development Phase or the KT activity, which is done as a sign of respect for the knowledge they hold (Lavallee, 2009).

For the third phase, participants were recruited via email. The principal investigator (CJD) identified key informants (i.e., those who were most involved in the project) and emailed each participant to ask if they would be interested in engaging in an individual interview to "close" the project (see Appendix F for Key Informant Email Recruitment Script). The principal investigator (CJD) already had a relationship with each key informant and, therefore, email was considered to be the best recruitment method for this phase.

Tool development phase. Three focus groups were conducted in a talking circle format. Prior to each focus group, a member of the research team (CJD) explained the study, requested and obtained informed consent (see Appendix G for RASS Tool Development Consent Form). As well, an Elder was recruited from RASS to facilitate the opening and closing ceremony for the talking circle and a smudging ceremony took place (a smudging ceremony is cleansing through burning a traditional medicine, such as sage, and passing it to each individual member of the circle). Elders were offered tobacco for their assistance as a sign of respect for their traditional knowledge (Lavallee, 2009). As well, participants were offered tobacco for their participation, which is a sign of respect for knowledge and is used to ensure that research is done in a good way (Baskin, 2005). Audio recordings began after the smudging ceremony. Two individual interviews were conducted similarly to the focus groups (i.e., smudging and offering tobacco), except there was no opening or closing and, for obvious reasons, there was no talking circle. The three focus groups and two interviews were approximately an hour and a half each and took place in a private and comfortable room at RASS. Food was provided at each focus group and interview, and participants were given the option of eating before or during the focus 
group or interview. Students were asked questions related to their perspectives on mental health; their knowledge about mental health facts, resources and coping strategies; and their preferences for receiving information about mental health (see Appendix H for Tool Development: Student Focus Group Questions).

RASS Staff members were asked questions about their perspectives on mental health, gaps in mental health knowledge among Aboriginal students and ways they have communicated information to Aboriginal students that was helpful (see Appendix I for Tool Development: Staff Focus Group Questions). Over the course of the focus groups, notes were taken by a member of the research team on a large flipchart for all participants to see and all responses were audiorecorded. Interviews were also audio-recorded, but notes were taken on the principal investigator's (CJD) notepad. Upon commencement of each focus group and interview, all participants were given $\$ 20$ as compensation for their time. The Aboriginal Elder who was interviewed was compensated $\$ 100$ for his time, as is expected in an Aboriginal context (Lavallee, 2009).

Knowledge Translation Phase. A knowledge translation strategy was developed based on the results of the Tool Development Phase. The principal investigator (CJD) and Aboriginal Elder who was recruited to co-facilitate the activity, worked together to develop the activity agenda. They met every two weeks for two months to discuss and solidify the activity topics based on the first phase results. Questions to be asked during the KT phase were first piloted in order to evaluate the appropriateness of questionnaires, so any issues could be corrected prior to evaluating outcomes (see Appendix J for KT Phase Pilot Questionnaire). Prior to piloting the questionnaires, the principal investigator (CJD) explained the study to participants, but did not 
obtain consent, as this was not considered by the Ryerson Ethics Board to be data collection, since specific feedback has not been reported in this dissertation.

An Aboriginal Elder as well as the principal investigator (CJD) facilitated the KT activity. Prior to the knowledge translation activity, a member of the research team explained the study, and requested and obtained informed consent (see Appendix K for RASS Knowledge Translation Consent Form). Participants were offered tobacco and the activity began with a smudging ceremony. The Elder provided an opening prayer. Prior to and after the KT activity, students were asked questions related to mental health facts, resources, coping strategies, and their perceived self-efficacy in managing their own wellbeing through a questionnaire format (see Appendix L for Wellbeing Knowledge Uptake Questionnaire [provided pre activity] and Wellbeing Knowledge Uptake Questionnaire [provided pre activity]). At the end of the KT activity, students were asked for their feedback on the appropriateness and helpfulness of the content of the activity, questions asked and the method of knowledge translation. Participants were also asked to provide feedback about what was helpful or not helpful about the KT strategy implementation as well as means of assessing knowledge uptake (see Appendix M for Post Wellbeing Activity Evaluation Questions). Details of the activity are presented in Results: Tool Development Phase as well as Results: Knowledge Translation Phase, since the development of this activity was considered to be the result of phase one and the implementation of the activity was considered to be the result of phase two.

To assess whether knowledge was being put into action as a result of the activity, participants were asked to complete an individual interview one month later, during which they were asked questions related to whether they remembered the information provided during the KT activity, whether they increased self-care behaviours, and what about the KT activity was 
helpful or unhelpful in increasing mentally healthy behaviours in their daily lives (see Appendix $\mathrm{N}$ for Knowledge to Action Interview Guide). One-month follow-up was chosen because this timeline has been used in the knowledge translation literature (Sudsawad, 2007) and a community member recommended that participants go through one full moon cycle before follow-up in order to allow the knowledge to be fully taken in (a moon cycle is a month's time; Personal Communication with R. Koleszar-Green, February 2013). All interviews were conducted in a private and comfortable room at RASS and participants were compensated $\$ 10$. They were also offered tobacco during this interview. It was decided that a community member would help analyze the data from the Knowledge Translation Phase and, due to the increased sensitivity in responses from the phase (relative to phase one data), all participants from this phase were re-consented to ensure that they were comfortable having a community member help with data analysis and interpretation (see Appendix O for Re-consent Agreement: Knowledge Translation Strategy)

Follow-Up/Debriefing Phase. Individual interviews were conducted with key informants to the project. Prior to each interview, the principal investigator (CJD) explained the study, and requested and obtained informed consent (see Appendix P Consent Agreement: Key Informant Interviews). Questions asked were related to participants' impressions of project, reflections of the research process, how they saw their role in the project, whether they thought that community needs were addressed through the project, and their thoughts on the current and future state of student mental health knowledge (see Appendix Q for Key Informant Interview Questions). The interviews were approximately 30 to 45 minutes and took place in key informants' offices or a place of their choosing. Interviews were audio recorded. Participants were offered tobacco prior to the interview and were compensated $\$ 10$. 


\section{Qualitative and Quantitative Analysis}

Regarding both qualitative and quantitative analysis, methodological and personal reflexivity were incorporated to inform the interpretations of all data. Historical and social contexts of the participants and well as the intended audience of this dissertation were considered in all discourse interpretations. Additionally, the writer's personal values and perspectives were considered throughout discourse interpretations as well as how these perspectives and values evolved throughout the data analysis and interpretation process. Both methodological and personal reflexivity were represented by the interpretations outlined in the results sections.

Tool Development Phase. Data collected during the Tool Development Phase was analyzed qualitatively using thematic analysis to understand mental health from an Aboriginal worldview, Aboriginal students' knowledge about mental health issues as well as ways to most effectively provide information about mental health to Aboriginal students. Transcripts of focus groups and interviews were treated as texts of experience and were not taken as objective truth or reality. Qualitative analysis was conducted using NVivo 8.0. Responses were analyzed using thematic analysis using the recommended steps above (i.e., Braun \& Clarke, 2006) and through incorporating immersion/crystallization, editing, legitimizing and corroborating (as is described by Crabtree \& Miller, 1999).

Immersion/crystallization is a common organizational style used in qualitative research (Crabtree \& Miller, 1999). When using immersion/crystallization, the researcher immerses himself/herself in the text and emerges after crystallizations, or reportable themes have been decided (Crabtree \& Miller, 1999). Editing is an organizational style that complements immersion/crystallization, and can be referred to as the way in which one would immerse him or herself in the qualitative data. When using editing, the researcher enters the text (immersion) like 
an editor, rearranging portions of the text into different categories until a meaningful summary has emerged (i.e., crystallization; Crabtree \& Miller, 1999). The editing process facilitated the development of a codebook, where the themes that began to emerge were assigned a code name. From this, two coders used the codebook to organize text exerts from the transcripts into meaningful categories (see Appendix R for Final Tool Development Phase Codebook). A reliability analysis was not calculated to ensure agreement because social constructionism assumes that different realities and perspectives are inevitable and, therefore, it was expected that the two coders would code things differently at time. Two coders were used in order to facilitate a discussion about the data to develop a rich story regarding the results.

The process of crystallization was finalized through corroborating and legitimizing, which is a critical step in qualitative analysis. This is where a research team meets to discuss findings in order to confirm and justify what has been found (Crabtree \& Miller, 1999). The two coders (CJD and SM) met after coding each transcript to have an iterative discussion about the themes that were emerging through immersion/crystallization and editing. Multiple meetings were required to crystallize themes that would guide the development of the knowledge translation activity and once a draft of these findings was written, they were provided electronically to all participants with the option for them to provide feedback via email or to meet in person to discuss the findings. No one provided feedback on the results either electronically or in person and up to two reminders were sent to participants.

A combination of Braun and Clarke's (2006) guidelines and Crabtree and Miller's (1999) recommendations were used to facilitate analysis. Specifically, the principal investigator (CJD) and a research assistant first became familiar with the data through data collection, transcription and re-reading (i.e., immersion). They then came up with initial codes (editing and 
crystallization), coded the data, reviewed the themes coded, defined the codes (crystallization, corroborating and legitimizing), and further crystallized their findings through writing the results and requesting feedback from participants.

Knowledge Translation Phase. Data collected during the KT Phase was analyzed qualitatively and quantitatively and from an evaluation/critical realist lens, rather than a social constructionist lens. Participants responded to the knowledge uptake and self-efficacy questionnaire and an average 'knowledge' score and average 'self-efficacy' score were calculated pre-KT activity and post-KT activity to measure the change in mental health knowledge and self-efficacy. A pre/post comparative analysis was not conducted due to the small sample size. The feedback gathered at the end of the KT activity and the responses gathered during the individual interview held a month later were analyzed using the same qualitative analysis process as outlined above (i.e., thematic analysis using immersion/crystallization, editing, legitimizing and corroborating as well as Braun \& Clark's 2006 step-wise guidelines; see Appendix S for the Knowledge Translation Activity Evaluation Codebook and the Knowledge Translation Activity Follow-up Interview Codebook). Quantitative data were collected during the one-month follow-up interview and average scores on these items were calculated. The principal investigator (CJD) and a research assistant (NW) analyzed this data. Due to the lens through which data were collected and analyzed being critical realism, reliability was calculated. Inter-rater reliability was greater than $90 \%$ between the two coders for the immediate feedback as well as the 1-month follow-up interview coding. Kappa was also calculated for both codebooks and was found to be over .75 for both the immediate feedback as well as the 1-month follow-up interview. Once a draft of these findings was written, they were provided electronically to all participants with the option for them provide feedback via email or to meet 
in person to discuss the findings. No one provided feedback on the results either electronically or in person and up to two reminders were sent to participants.

Follow-Up/Debriefing. Data collected during the third phase was qualitatively analyzed using the same analysis process as outlined above (i.e., thematic analysis using immersion/crystallization, editing, legitimizing, corroborating and using Braun \& Clark's 2006 step-wise guidelines; see Appendix T for Final Follow-Up/Debriefing Phase Codebook). The principal investigator (CJD) and a research assistant (NW) analyzed this data, but a reliability analysis was not calculated to ensure agreement due to the reasons outlined above as related to social constructionism. Once a draft of these findings was written, they were provided electronically to all participants with the option for them to provide feedback via email or to meet in person to discuss the findings. No one provided feedback on the results, either electronically or in person, and up to two reminders were sent to participants. 


\section{Results: Tool Development Phase}

The results from this phase are presented through a description of metathemes as well as themes found to fall under each metatheme. Not all participants spoke to each theme. See Figure 1 for a visual representation of the results described below. It should be noted that initials have been placed after each quote to reference each participant. In order to protect participant confidentiality, the initials used are not that of each participant's name. Responses from students and staff are presented. When both students and staff have spoken to a particular theme, student's responses are presented first and then staff responses are presented.

\section{Definition of Mental Health from an Aboriginal Perspective}

This metatheme represents a definition of mental health from an Aboriginal perspective. The following themes, taken together, define mental health from an Aboriginal perspective. The specific themes that capture this definition are described in detail below.

Mental health is inseparable from one's self. Students and staff agreed that mental health is only one dimension of the whole person and, therefore, cannot be separated from the self. More specifically, students noted that emotional, mental, physical, and spiritual components interact with one another to affect one's wellbeing and that mental health cannot be disconnected from these other elements. The following quotes demonstrate students' view of mental health as a wholistic concept: "I was thinking for like me, like I think of mental health... as a heading...if we were to put them in the medicine wheel, like kind of do the wholistic approach, like they [Aboriginal people] would put the emotional, the spiritual, the physiological and then the mental" (NH), "well, in an Indigenous perspective I think of mental health as being the whole person so like your physical, emotional, spiritual" (BG), and "So Aboriginal perspectives are more wholistic views, so they think about the mind, the body, the spirit..." (LV). As is clear 
from these quotes, students constructed mental health in relation to the physical, emotional and spiritual aspects of the self, supporting the idea that these aspects are not perceived as being in isolation or separate from one another. This idea aligns with literature describing mental health in this way, including writing from Alberta Mental Health Board (2006), Blackstock (2008), Isaak and Marchessault (2008) and NACCHO (1993).

A final quote from an Aboriginal student demonstrated the invalidation of Aboriginal worldviews, and that Aboriginal knowledges, as related to common wholistic views of mental health, is rarely acknowledged: "It's like, I'm not sure if enough attention is being paid to the wholistic part, other than just kind of the tip-of-the-hat tribute to it. But it's like, where is the actual like work being done when they're tying them together to show that they are connected, you know?" $(\mathrm{NH})$, This participant is referring to a lack of acknowledgment of Aboriginal worldviews, which reflects the common invalidation of Aboriginal knowledge and worldviews in a positivist Western context, as well as the impact of colonization on Aboriginal communities, as many teachings and traditions have been lost over generations. This participant seemed to suggest a push for restoring such teachings within Aboriginal communities as well as recognition and acknowledgement from Western contexts. This is in line with Battiste and Henderson (2000) who discussed the difficulty of Westerners in recognizing Indigenous knowledge due to the positivist Western need for definitions. Indigenous scholars have not established a definition of Indigenous knowledge, yet precision and clarity is a common quest within a Western context. Battiste and Henderson (2000) discussed how the quest for clarity is motivated by Eurocentrism, which assumes the superiority of Europeans over non-Europeans and that European beliefs are viewed as 'true', and as supported by the 'facts' or seen as 'reality'. Battiste and Henderson (2000) suggested that Indigenous worldviews and Eurocentric worldviews cannot be compared 
until "Eurocentric thought is able to qualify the principle that categories are constructed by human minds for human purposes" (pg. 37). This further demonstrates the differences in Aboriginal and positivist Western worldviews and may also reflect the above participant's frustration regarding the lack of acknowledgement of Aboriginal worldviews within a positivist Western context. In line with the participant's above quote is Battiste and Hendersons' (2000) call for acknowledgment and a restoration of Aboriginal culture and teachings.

Similarly to students, staff agreed that mental health is merely one dimension of the whole self and how, when the mind is not balanced, everything is unbalanced, as the mental, physical, emotional, and spiritual aspects are all interconnected. These ideas are demonstrated by the following quote: "For me growing up in my community, my understanding of mental health is that it is part of who we are. It's one component of how we grow, and it's an addition to our mental capacity, and our physical capacity, and our emotional and spiritual capacity" (MK).

There was agreement among staff about how achieving balance within mental health and between all of the other elements of the person is desirable, but not possible and that one must always be working toward such balance, as demonstrated by the following quotes:

For me, when I look at mental health, it's much more of a wholistic understanding. And that it's a spectrum. And that it's something you're trying to balance. And in that achieving balance is a fallacy. And so that, at some point, you may be in a healthier space or not a healthier space and it's a constant way of engaging the world. (GR)

This participant positions balance as something that can never be achieved, which aligns with the circular aspect of Aboriginal culture. Time is often perceived as circular within these context, which is in contrast to Western contexts, where time is viewed as linear and, therefore, there is an assumption of a beginning and an end from a mainstream perspective (Smylie et al., 2000). The quote above draws attention to Aboriginal perspectives of time, since the assumption is not that you will achieve balance and that is the end, but rather it is something to be striving 
for on a daily basis and throughout one's life in a circular way that is similar to perspectives on time as well as on many other phenomena from Aboriginal perspectives. This also taps into the idea that people are always learning and growing and that this never stops because, if people achieved knowledge about everything possible, there would be no point in continuing on with life (growing and learning is part of life; R. Koleszar-Green, personal communication, July, 2009). The following participant also spoke about perspectives of balance:

It's not about achieving balance, it's about knowing that it's there. As [participant name] was saying earlier, it's a spectrum. It's when all of those things get so far away from me that I'm not able to look at them and be honest with myself, with what's happening inside me. (MB)

The latter part of this quote speaks to the element of self-awareness, as well as honesty in one's self-awareness, as being key in striving for a balance within and between all elements of the self, including the mental aspect. There was further acknowledgement of the importance of self-awareness with regard to striving for balance and maintaining wholistic health in the following quote:

It's critical that we have self-awareness. It really is how it is you are able to greet every day and figure the things you are carrying in your life and take a look at your responsibilities and check in. Have you had enough sleep? Have you eaten properly? Are you saying to the people around you what you are thinking and feeling? (MK)

The concept of self-awareness and checking in with oneself also relates to the concept of self-determination, which is a common theme in Aboriginal contexts and emphasizes a person's or community's right to make decisions for himself, herself or themselves without external forces (e.g., governmental forces; Asch, 2002). A major goal for many Aboriginal communities is to strive for self-determination, so that outside governmental influences become minimal and Aboriginal Peoples can be responsible for their own political, correctional, school, and employment systems (Asch, 2002). Self-determination can also be applied at the individual level, as one may strive to be responsible for oneself and not feel the need to rely on the mainstream 
system or governmental influences to make decisions for their own wellbeing (Napolean, 2005). In sum, self-determination at both a community and individual level is the ability to control one's destiny (Asch, 2002; Napolean, 2005) and self-awareness could be seen as a pathway through which one achieves wholistic wellbeing.

Mental health is a community responsibility. This theme captures the idea that mental health is not just an individual responsibility, but also the responsibility of one's community. Staff drew attention to bringing different people together to target different aspects of yourself so that you can attend to your whole self, “...It's always important to bring all of these people together because sometimes it can be too easy to keep them all apart. Then you're still not attending to your whole self" (MB). This quote relates to the concept of self-awareness, since bringing these different people together can heighten one's awareness about their wholistic wellbeing, and promoted the idea that one doesn't have to be alone in, or solely responsible for his/her self-awareness or wholistic wellbeing. This idea begins to tap into the connection of mental health to other people and to one's community. The concept of mental health as a community responsibility is further elaborated below.

Being willing as an individual who acknowledges mental health in this flowing and organic way is being able to also move between where you are located in that circle. You may be the person right now who is in the middle of that circle and you have everyone around you, helping you, giving you those supports, but then you need to step out at some point and you're going to be in the outer circle giving that to someone who is going to move in (MK).

Right, and having that flexibility that you can move in and out of that circle fluidly. That's the Aboriginal way; it's not a western way. (MB)

Staff used a metaphor of a circle above to describe how mental health cannot be divorced from others in the community, as such difficulties will always be dealt with in the context of other people and as the person who may be struggling, will be placed in the middle of the circle. It should be noted that circles are commonly used within Aboriginal culture to represent equality, 
interconnectedness, strength, and continuity (Ontario Curriculum, 2006). As illustrated in the above quotes, there is an assumption within an Aboriginal worldview that mental health should not be dealt with in isolation; rather, it is expected that others will surround the person who may be struggling with one or more aspect of themselves, in support of that person. That same person, in the middle of the circle, will eventually leave to become a part of the circle and offer his/her support to whoever has entered the circle and is struggling. People are constantly moving in and out of the circle, helping and being a helper in a fluid and organic way. This relates to the emphasis on community that is very much in line with the Aboriginal way (Smylie et al., 2003). It suggests that there is fluidity regarding who is being helped and who is helping, as community members may transition from one role to the other, depending on what the needs are at the time. Overall, the struggle one may go through is considered to be a community responsibility.

Mental health is passed on intergenerationally. Students and staff agreed that mental health is a relational concept, meaning that the mental health difficulties with which Aboriginal people face have been passed on from generation to generation and there is an assumption that the Western mental health system (and society) does not understand this, creating a disconnect when trying to access services in the mainstream system. This is demonstrated by the following student quote:

I always feel like when I go to a non-native person [for counselling], I'm kind of holding back some information. The reality is that she hasn't lived what I've lived. She doesn't understand intergenerational trauma the way that I do. So when I go in there and say one thing to her, now I have to explain why I think that way or why I feel that way. So now I've gotta explain to her why I live this way or why that is my norm. It's because Western ideology is different from our perspective, because a lot of that comes back to colonization. That whole, breaking us down as a people, that has a lot to do with it. So even though, it's like yeah okay, my grandparents didn't go to residential schools but you know their parents did. You know, just because that wasn't in my family really close, it doesn't mean it didn't impact us. The reality is that...you know, we're just as impacted because...we are all ashamed of that stuff right? The reality is, we shouldn't be, because 
it's not our fault. You know what I mean? It's like even that shame and that guilt of like who we are as a people is shared through the generations. Right? (NH)

The concept of shame in the latter part of this quote demonstrates how the wellbeing of Aboriginal Peoples is significantly affected by intergenerational trauma, as not only are mental health issues being passed on through the generations, but also the shame of being Aboriginal is passed on, as being shameful of one's Indigenous background was taught to Aboriginal people throughout colonization (Morgan \& Freeman, 2009; Indian and Northern Affairs Canada, 1996). The concept of shame being passed on intergenerationally relates to a theme that will be discussed below regarding Aboriginal identity, as shame in one's Aboriginal identity has also been passed on intergenerationally. The above quote taps into the idea that there is a lack of understanding, within a mainstream context, of the history from which Aboriginal people are coming, which contributes to struggle and frustration among Aboriginal people when accessing services. In addition to mental health concerns and the shame of Aboriginal identity being passed on intergenerationally, there was agreement that certain life skills were not passed on due to colonization, which impacts mental health and wellbeing, as demonstrated by the following student quote:

...on the average people are coming like from non-Aboriginal backgrounds...often from more healthy environments where certain like life skills have been developed over generations...that practice of not talking about your feelings and not seeking help and the stigma that really is with the non-Aboriginal culture. Then what's happening is that Aboriginal people start adopting that for themselves but we are coming from a completely different place: from residential schools, from the 60's scoops, from all these kinds of traumas. Why do we have such high suicide rates, addiction rates, and incarceration rates? Cause it's like we are applying the same rules to our game when we are in a completely different game than everyone else (laughs). It's messed up right? $(\mathrm{NH})$

The quote above represents a construction of Aboriginal and non-Aboriginal people as coming from two completely different "games" and yet, since Aboriginal people are often 
located in the Western system, they are left to apply non-Aboriginal "rules" to their very different Aboriginal context (or "game"). There is an assumption in the above quote that nonAboriginal people are coming from healthier environments, where effective life skills and coping strategies have been developed and passed on throughout the generations. Therefore, stigma and shame as related to mental health is assumed to be a cultural norm within a non-Aboriginal context, since there are fewer of these issues, especially since non-Aboriginal people have very different/healthier histories. It is assumed in this quote that non-Aboriginal people may have been protected from mental health issues in some way. The quote also suggests that, when stigma is applied to mental health issues, it can create pain, in addition to the mental health issues with which they may be struggling, especially since there is much cultural restoration that is sought by Aboriginal people, due to the intergenerational trauma with which they have faced. Applying stigma to an Aboriginal context is not the traditional way and serves to continue to oppress Aboriginal populations, while reinforcing mental health issues within these communities. In sum, the application of stigma to mental health within an Aboriginal context is rooted in a history that is specific to Aboriginal Peoples and, therefore, has a negative impact on these communities due to the vastly unique historical contexts from which they are coming and the fact that stigma never existed, traditionally within Aboriginal contexts.

Gone (2007) supports the idea that Aboriginal People and non-Aboriginal people are coming from two completely different 'games' with a focus on historical trauma as creating a very different context from which Aboriginal Peoples are coming, significantly impacting mental health. Gone (2007) interviewed an Elder from the Northern Plains Indian reservation in the United States, who conceptualized colonization as the problem and cultural revitalization as the solution to personal and communal disorders and distress (Gone, 2007). 
Similarly to students, staff agreed that negative pathologies are passed on and experienced through colonization and that Aboriginal people are abused in various ways on a daily basis, which stems from colonization and contributes to the historical and present assault on traditional spiritualism and identity issues. This is demonstrated by the following quotes, which emphasized the issue of forced relocation as a result of colonization for Aboriginal Peoples:

So let's unpack that [mental health] in terms of generational trauma. We have communities that have been removed from their traditional territories. When you remove people, you disrupt the relationship with land, with food gathering, with ensuring your body is receiving nourishment that it needs from the land. So you can't divorce your mental health from the fact that you may have lived for years in poverty. (GR)

This quote captures the policies that were put into place to forcibly remove Aboriginal people from their land, which greatly disrupted their ability to engage in traditional activities. Lost knowledge, as well as roles and responsibilities, continues the cycle of lost generations, which will undoubtedly affect mental health issues. Aboriginal people have traditionally had a strong physical and spiritual relationship with the land and many community members continue to have this relationship (Battiste, 2000). The land was (and still is) thought to give people what they need (i.e., food and nourishment). This participant has drawn attention to the disruption of the relationship between Aboriginal people and their traditional lands through colonization and how this has impacted mental health even through the generations. Today, Aboriginal people continue to fight to hold onto the little land they have left (Evans, 2014). There is currently legislation that has been recently initiated (e.g., Bill C-45; Department of Finance, 2012) and, if passed, will contribute to even more loss of land among Aboriginal people (Paul, 2012). The policies positioned as having an intergenerational impact on mental health among Aboriginal people are further discussed below: 
When you put policies in place that penalize people in how they get their own food, or when you penalize people for picking up their spiritual practices, all of those over time, the kind of messaging that it gives to people makes it more challenging for people who did not grow up in communities where they were taught about all of those roles and responsibilities. You have to create the space for them to be in touch and learn those teachings in a way that makes sense for them. (GR)

This quote demonstrates the issue of forced relocation as well as policies that have disrupted Aboriginal culture and their sense of identity. Through policies that were put in place to restrict Aboriginal people from practicing their traditions and culture, Aboriginal people lost their sense of traditional roles and responsibilities and, therefore, have had difficulty passing this knowledge onto future generations (Kirmayer, Simpson, \& Cargo, 2003). Through their inability to pass on traditional knowledge, Aboriginal people are left with a lack of culture to turn to for coping/healing, which cannot be separated from mental health concerns (Connors, 2007; Little Bear, 2000).

Staff explained that the historical unsanitary and even dangerous work environments in which Aboriginal people were/are often situated, contributes to adverse physical health conditions, which cannot be separated from mental health or the other components of the self. Accordingly, all components of the self have been affected by these conditions and have been passed on to future generations:

When our bodies get cancer, when you think that we [Aboriginal people] have the highest rates of heart disease, those are put into context when you start to take a look at what has happened over time. I guess the correct term would be 'environmental factors'. You think about where our people have had to work and the fact that they probably weren't given the information to do that work safely. Anybody in the boating industry, the asbestos that was there, or you think about anyone who worked in logging, the physical ailments that come with that. (GR)

In order to provide clarification about the impact of Aboriginal histories on mental health, staff drew attention to survivor guilt and trauma that has been passed on intergenerationally through blood memory, which is being linked to physical trauma, degradation of living and 
working conditions, as well as elimination of food sources, which will all impact mental as well as physical health among Aboriginal people, presently. Blood memory refers to how memories are stored in the genetic makeup of one's body (J. Dallaire, personal communication, October $7^{\text {th }}$, 2013): "Young Blood has a term about the guilt, survivor guilt, and how that survivor guilt is passed down from Indigenous body to Indigenous body and the beliefs in blood memory and the trauma and the PTSD has been almost embedded in our blood memory". This quote demonstrates how, even when someone has not experienced trauma first hand, they are still impacted by such trauma through the phenomenon of blood memory. The concept of blood memory has been explained as the need to care for generations who have lived through trauma, rather than having one-off approaches to treating trauma because entire communities have been left vulnerable and traumatized (Maracle, 2011). This is further demonstrated by the quote below:

And no matter how we work on ourselves, everyone is going to be impacted by intergenerational trauma. They are going to carry their own understanding of intergenerational trauma, which will be affecting their mental health no matter how much we heal ourselves. (GR)

Both quotes demonstrate how mental health issues are passed on intergenerationally. This quote emphasized how Aboriginal people will always carry historical trauma with them and how this trauma cannot be separated from mental health concerns or any other aspect of the self, as the impacts of historical trauma are unavoidable for Aboriginal Peoples.

Although the majority of Aboriginal students and staff spoke about the adverse impact of intergenerational trauma on the mental health and general wellbeing of Aboriginal people, one staff member expressed his hope for future generations. In working with students, this staff member talked about how he hopes for a day when Aboriginal students come to him with only academic struggles, rather than all of the other issues that he commonly sees, including mental 
health concerns, that have been passed down intergenerationally. This staff member reported that he is already starting to see some disruptions in the cycle of intergenerational trauma. This idea is in line with a theme of change. With the movement of Idle No More and the advocates working for Indigenous rights, there have been considerable changes over the past decade, which may be working to disrupt the cycle of intergenerational trauma (Idle No More, n.d.; Smith, Varcoe, \& Edwards, 2005) and this may be reflected in the changes observed by this staff member.

Identity is central to mental health. This theme was constructed by students and staff, since identity was discussed as playing an important role in one's mental health and wellbeing. More specifically, students commented on the difficulty of understanding what it means to be Aboriginal and how this can contribute to mental health struggles. An example of this comes from a student who described her struggle with understanding her Aboriginal identity, “...there is like this conflict in identity of, you know personally, I've been brought up in a Westernized society and yet I have an Aboriginal background and Aboriginal relatives. It's just sort of conflicting ideas of who I am...” (HJ) This participant points to an internal conflict that is created for her because of the lack of clarity in her own identity, which can manifest through struggles with mental health and wellbeing (Chandler \& Lalonde, 1998), and is further demonstrated by the quote below:

I was raised as a Roman Catholic until I was 18 years old, 19, and I had a nervous breakdown (inaudible) because it didn't make any sense to me after a while. Then I went back to Native culture and I got grounded back in reality... because I found myself after a while, eh? Because I had lost my identity. (RT)

The above quote further shows the connection between identity and wellbeing. In particular, this participant's reference to a "nervous breakdown" illustrates how such confusion can cause a build up of struggle that may eventually require a release. Such a release, in this 
example, allowed this participant's identity to take its rightful place and piece itself together again.

Similarly, staff spoke about how denying your Aboriginal identity can create mental stress and that people need to be proud of their Aboriginal identity. Staff felt strongly about the necessity of Aboriginal people being able to claim their identity and "own it". Staff discussed identity issues as being closely tied to suicide, since there are policies in place that communicate to Aboriginal people that they are not supposed to exist. This message becomes incorporated into Aboriginal peoples' identities, contributing to high suicide rates. Staff noted that when you have your identity, you can accomplish great things in your life: "And how did [Aboriginal participant's name] get through? Knowing who she is, so it's about identity as she said, but it's also about having those circles of people around her. If [participant's name] didn't have those spaces or that identity or the parents she had, this space would not be existing" (GR). This participant highlighted the importance of having support in your life and one piece of support can be knowing your identity.

One staff member specifically connected the idea of how identity issues as well as pride in one's Aboriginal identity can impact mental health:

Because if you cannot relate to your identity, it's going to create a little barrier and if you don't address the barrier, it is going to get bigger. I have students here that...feel really uncomfortable because of the fact that they have hidden it [their Aboriginal identity] for so long until they think 'wait a minute, I am proud of who I am' and when they do they get that reverse (laughs)...that creates a little bit of mental stress on them...Because if you were out there and you are not comfortable with what's in your skin, you are going to have a hard time, you know? (SE)

The above quote suggests that denying one's Aboriginal identity can create mental stress, and upon embracing their Aboriginal identity, after denying it, this will create mental stress as 
well. However, one can eventually become comfortable in their own skin and with their identity, which can contribute to an increase in one's wellbeing, which is further demonstrated below:

Ya, I had one student who didn't look that Native, her Dad was Ojibwey and stuff and she is fiercely proud of that, but she could have went and gone right with the mainstream but she said 'you know what [participant's name], my mom always encouraged that I keep that part of me' and that the relationship she had broken up... because her boyfriend wanted her to keep quiet about her you know [identity] and she said, 'that's not worth it, I want to be happy and how can I be happy if I'm told to...[keep quiet]'. So I said 'how did that bother you?' She said, 'mentally, I was losing sleep!' I goes 'Was it worth it?' she said 'no'. So break up. It hurt, but you know what? In time you get over it and you work on it, but she said 'the fact is, I am part [Native] and that will never leave me and I got to work with that', and she has now come to ceremonies that I suggested and I am really proud of her, so that alleviated her mentally, emotionally, physically and spiritually. (SE)

The above quote demonstrates the mental stress that can be created when one denies their Aboriginal identity as well as when one decides to embrace their Aboriginal identity. This quote also shows how such mental stress can be alleviated through embracing one's identity over time and how this can promote wellbeing in all elements of the self (i.e., mentally, emotionally, physically, and spiritually). The beginning of this quote taps into an issue within many Aboriginal communities of being "not Native enough", perhaps because of how one looks or how connected one may be to their Aboriginal culture. Feeling as though one is "not Native enough" can contribute to an identity struggle and will require a process of accepting the fact that one may lack stereotypical visible evidence of their Aboriginal identity (e.g., having lighter skin, blonde hair) or the fact that they have been raised without their Aboriginal cultural and traditions. However, in spite of lacking particular physical characteristics as well as experience with the culture, this participant is noting that Aboriginal identity can still be embraced and reconnected to. Aboriginal culture has been described as traditionally inclusive and, therefore, it would be a rarity to require people to prove their Aboriginal ancestry in Aboriginal contexts; however, in some circumstances this is the case and lateral violence or hostility still exists 
toward those who don't "look" Native. This is in line with Gone (2006) who suggested that people have difficulty understanding that "Indianness" (pg. 58) is something that you are, not something that you do or how you look, because of the stereotypical portrayals of Aboriginal people in the Western media (e.g., as stoic figures in feathers and paint who dance, ride horses and hunt with a bow and arrow).

Staff drew attention to how going through mental struggles contributes to who you are as a person and strengthens your self. They discussed how mental health is part of who you are and, because of this, it is not curable, as is commonly the goal in a Western context.

Sometimes in that space [University] you come to a push and pull that can make someone look at something they've pushed down for a long time. And that can happen in a lecture. Something that an instructor is saying or it can come in a book you are reading or research you are looking at, but it's sending you a message that is going against something within you that you need to attend to. So it can be for a day or a couple of months or it could be for the rest of your life. If you put the right supports around that person to assist the person in understanding, yes you can deal with it right now but maybe after we pull all the people around you who need to be around you, you may have to live with this for the rest of your life. And so having that space and time to think of how they can carry and add this into my bundle of who I am. (MK)

The above quote references how a University setting is a space where people are learning and growing, which can trigger struggles in people and such a struggle can relate to various issues, including but not limited to the lack of Aboriginal content or inaccuracies of Aboriginal content in post-secondary curriculum (Baskin, 2011; Battiste, 2000). The quote speaks to the supports that one may seek if they are triggered, but also how these struggles may need to be accepted as part of one's identity, which is noted by referencing a "bundle". "Bundle" is a term used in Aboriginal contexts that is closely related to the term "identity". First Nations people may have sacred items to help guide them (Anishnawbe Health Toronto, 2000a). A personal bundle can consist of these sacred items (e.g., rattle, eagle feather, pipe, or a drum) and a person 
is responsible for taking care of each item in their bundle (Anishnawbe Health Toronto, 2000a). Their bundle aids in personal development (Anishnawbe Health Toronto, 2000a).

Both students and staff agreed that language is an important part of knowing your identity and who you are as an Aboriginal person. One staff member stated that, "Language is always part of how we learn and who we come to be as part of our identities" (MB). Therefore, knowing one's heritage language may be helpful in reducing mental stress and promoting wellbeing, as language is considered to be intertwined with one's identity, especially in an Aboriginal context (Battiste, 2000).

Stigma is closely tied to mental health. Students and staff spoke about the stigma that is associated with mental health and how this is very much a part of their perspective on mental health, as stigma for Aboriginal people is rooted in a different historical experience, as was discussed above. More specifically, students spoke about feeling embarrassed about seeking help for a mental health issue: "Ya, just like even to go, like when I was younger I would have felt like embarrassed to say I need to go and see like a counsellor..." (TL). This quote taps into the difficulty in accessing help with struggles due to the stigma that surrounds mental health issues, even though traditionally, it was expected that people reach out for help, rather than deal with their concerns in isolation, as was noted in the quote above.

Students positioned mental health as a weakness: "Back where I'm from there are still some people who maybe treat it as humour, I don't know like negative humour that's associated with that, you know, and that has a lot to do with some of the things you talked about like misunderstanding and weakness" (LV). This participant notes that many people do not take mental health issues seriously and, therefore, treat these issues as humourous, which can further 
contribute to the stigma around mental health concerns. The following quote also positioned mental health as a weakness that is highly stigmatized:

Ya, and I think there might be something in there with like a stigma associated not just with being having mental health issues but seeking treatment so um and it has to do with trust issues with doctors and health professionals, so I don't know, there is a feeling like why should I go to the doctor? Like there is a trust issue thing there in Aboriginal context at least from my perspective. So if you go to a doctor, you are weak, you should just deal with it yourself like um, ya. (DN)

This participant draws attention to how the stigma around mental health is so strong that people do not want to seek help, but if someone is thinking about getting help, there needs to be trust. This participant noted that trust is an important element of mental health stigma because without it, the stigma can overpower one's ability to access necessary resources. Trust for people who are located in a Western system may be difficult to develop for Aboriginal people, since this is the same system that historically traumatized and continues to oppress Aboriginal Peoples. There may also be reluctance in trusting Western professionals to be able to help Aboriginal people due to a general lack of understanding that the Western system has about Aboriginal contexts, which is further discussed below. Finally, the idea of trust is in line with Aboriginal culture, as this context heavily emphasizes relationship building and relationship development is closely tied to the presence of trust (Smylie et al., 2003).

Related to the connection between "weakness" and mental illness as contributing to mental health stigma, some students perceived mental health as something that should simply not exist: "Ya, a lot of people think of it [mental health] as a weakness or a vulnerability you know, you shouldn't be struggling with mental health problems" and “...I guess the way I grew up on the reserve, there's a lot of stigma, you know? So a lot of it is depression, anxiety, stress, they're like, 'oh just get over it'. So I guess I try to help myself' (DN). The latter quote suggests that there is general agreement that people should not be struggling with mental health concerns (i.e., 
"get over it") and also how this stigma can contribute to having to cope with mental health concerns in isolation (i.e., "I try to help myself"). Breaking the cycle of silence and isolation around mental health is something that is gaining momentum in the mainstream system with initiatives such as 'Bell Let’s Talk', where Canadian Olympic medalist, Clara Hughes has spoken publicly about her difficulties with mental health in order to promote awareness and help others to refrain from dealing with their difficulties in isolation (Bell Canada, 2014). As well, Manitoba Chief Phil Fontaine has spoken publicly about the effect that residential schools had on his wellbeing and his struggles as a result of attending residential schools (Enright, Maitland; interviewers, \& Fontaine; interviewee, 1990).

Students positioned mental health issues as something that should not be talked about due to the stigma around it: "It has a lot to do with mental health or even saying you're gay or trans or bi, there's just certain things on the reserve that you don't disclose". This quote points to the perception that mental health stigma is similar to the stigma around sexual identity and may reflect the idea that mental health is static/permanent, as is sexual identity, rather than something that can be "cured". This quote also draws attention to mental health stigma in small towns, like reserve communities, where everyone knows everyone. Due to the stigma around mental health, there is a lot of risk in disclosing mental health information, but especially in a reserve community where one's reputation could be at stake. This small town issue (i.e., many people having relationships with one another due to the small number of people residing in one geographic location), as related to stigma, is further demonstrated by the following quote: "Also, seeking treatment in the Aboriginal community, it's so small and people know a lot of people, so I think that's another issue with seeking treatment that if we want to go to an Aboriginal agency, it's so small that everybody knows everybody kind of thing" (DN). This participant is expressing 
her concern and mistrust in the ability of mental health professionals to maintain privacy and confidentiality if treatment for a mental health condition was sought.

Related to the topic of keeping quiet about one's mental health struggle is the following quote: "And it sucks because then you can't get help and it leads to all that other stuff", further suggesting that the stigma can lead to other issues related to mental health and wellbeing or exacerbate a current mental health concern through a lack of access to resources. Through a lack of resources, mental health concerns can worsen or feed into other mental health issues, which can contribute to additional concerns with physical, emotional and spiritual health.

Students spoke about having the mental health labels compound with stereotypes associated with being Aboriginal, which can make the stigma even stronger and more difficult to deal with, further contributing to mental health issues:

And I think that for Indigenous people already there are so many labels and stereotypes already that it [mental health labels] kind of compounds with that and can kind of make it even worse. Ya, and similarly for people not going for conventional treatment, I think there is more self-medication too. That has been my experience. (KB)

This quote specifically suggests that the interaction between mental health stigma and Aboriginal stereotypes may put Aboriginal people at risk for developing other mental health issues or trigger self-medication with substance use. This provides a potential explanation regarding why addiction issues may be so prevalent among Aboriginal populations (Teasdale, Conigrave, Kiel, Freeburn, Long, \& Becker, 2008) and mental health stigma as a precursor to addiction issues has not been regularly explored in the literature examining addiction issues or concurrent disorders among Aboriginal populations.

Staff spoke about how "mental health" as a term that has so much stigma associated with it that the term should not be used. Instead, the term "wellbeing" should be used:

...for Aboriginal students, I think it is really important that we almost not even use the words 'mental health', it's almost because of the negative connotations and for so many 
of us Aboriginal people we already feel like everything is negative about us. So wellbeing like as we have said changing language. Why are we not calling it wellbeing? (GR)

Similarly to students, staff discussed how people are not encouraged to acknowledge that mental health even exists, as people are not supposed to talk about it: “...but students and individuals are not encouraged to acknowledge that it [mental health] even exists" (MB). One staff member in particular, noted the incredible effort it can require to hide mental health struggles due to stigma, that it can begin to contribute to further struggles with mental health, creating a cycle of difficulties. “....if I didn't go through this, I wouldn't be the woman I am. I think when we try to hide and deny our mental health struggles it takes more energy and more struggle to hide the struggle" (GR). This participant calls for a different perspective on mental health concerns, such as viewing it as a lesson or teacher, contributing to one's growth as a whole person.

Cultural connections impact mental health. Students and staff spoke about how one's mental health is impacted by a dynamic process of cultural connection and reconnection. Cultural connection was described as impacting mental health, since when one feels culturally connected; they are able to move toward wellness. More specifically, students spoke about embracing their culture through sweat lodge ceremonies as well as other ceremonies so that their spirit can come alive. A sweat lodge ceremony is a cleansing, purification, or healing ceremony that is grounded in spirituality and takes place inside a structure and around a sacred fire (Anishnawbe Health Toronto, 2000b). Students specifically noted the necessity of taking care of their spirit for their own wellbeing:

...when I go to see...the Native community... the sweat lodge ceremony and even smudging if that was there...when we are doing these things, it's like we are embracing our culture, like I think our spirit like comes alive...to ask me to be able to describe it in words, how that was beneficial or like fancy words, I wouldn't be able to do that. It's 
something that's going on that's more than you can see, like with the eyes, you know? Cause I've found for myself...talking is good and everything, but it only takes you so far and I feel like some of the things that we do as Aboriginal [people] takes us the rest of the way, you know? Cause there is some talking, but there is some, like there's actions that we do and like rituals that we do and stuff like that..." (NH)

This quote demonstrates how connecting to aspects of one's culture can promote wellbeing through an internal process of healing. This participant also described it as being a process that cannot necessarily be seen, but can be so important for healing and how this may only be possible in an Aboriginal context.

Students also spoke about the importance of connecting to their Aboriginal culture in order to take care of the whole self, including the mental, emotional, physical and spiritual components:

Ya, cause it was at that point when I was doing all the research [about mental health], I realized that I was getting there and once my spirits were ok, then my emotions would be ok and then after that, all I would have to work on would be the physical aspect, so I hit the gym and then that helped raise my spirits as well. (TL)

The quote above shows how the different elements of the self are connected to one another and by taking care of the spiritual element, which is often through cultural connection, the other aspects of the self could be taken care of as well. Taking care of the whole self may be done through various means, including a combination of cultural connection and Western approaches such as going to the gym. The importance of cultural reconnection is also discussed below:

And it wasn't until later when I was introduced to like well when I sought out like my Aboriginal culture, right? And then it's like and then the medicine wheel was put up and like that whole wholistic approach was shown and it hit me and I was like this [mental health] isn't actually a part of myself that I'm supposed to be ashamed of you know? Like this is a piece of myself that I'm supposed to embrace you know? And if you have a problem like in the most obvious one like if you have weight issue you go to the gym you know? So that like that's just normal, that's just common sense, right? So that's when it really hit me that I was just like 'wow'... (NH) 
The above quote demonstrates the healing process through which one participant went by reconnecting to his culture. In particular, once he learned about his health and wellbeing from a wholistic perspective (e.g., using the medicine wheel), his worldview began to shift and he began to understand, as well as give himself space to understand, his struggles with wellbeing. It is also interesting that this participant spoke about the solution to a weight problem as going to the gym and not through decolonization, as weight issues for Aboriginal Peoples could be rooted in more than just a sedentary lifestyle. Weight issues could be rooted in historical trauma such as forced relocation or a lack of access to traditional foods, for example. This participant is adopting an individualized approach and might not be acknowledging the historical or broader context of Aboriginal people.

Students mentioned the importance of going to Aboriginal Elders for guidance and using circles to facilitate healing and communication in order to connect to their culture and promote their wellbeing:

...I think also in like bigger groups when you sit in a circle and stuff, I've experienced that too, so like sharing circles, people share a lot and a lot gets talked about so you know the great thing about that too is that we're all in it together we're all in a circle and we're facing each other and we're all intimate and we're all on the same level. (DN)

This participant is sharing how one could use their cultural connection to facilitate healing. The description above is of a sharing circle, which is a traditional practice where traditional medicines are present, everyone is considered equal, and everyone gets a chance to share (Ontario Curriculum, 2006). She specifically noted that "we're all in it together", which highlights the inclusion of community cohesion within the theme of cultural connection, since a cultural norm in an Aboriginal context is a connection to one's community (Smylie et al., 2003).

It was emphasized by staff members that, in order to promote mental health and wellbeing, one must seek out their teachings, as well as people, to help them connect and/or 
reconnect to their culture: "I will encourage you to go to your ceremonies. [Principal investigator's (CJD) name], if you could do that, you are going to wholistically develop in a way that there will be no stopping you. Go to your ceremonies, um always find someone that you can trust [to take you]" (SE). This staff participant is encouraging the principle investigator (CJD) to go to ceremonies for her own wholistic development and wellbeing. Staff spoke about the importance of going to ceremonies to alleviate mental stress and using spirituality to heal yourself:

So, that spiritually, they grab onto that [through ceremony], they realize 'hey, wait a minute, I could, I need this' and that way they are all interconnected. You become centered because how can you be balanced when one of those entities are off balanced? I think of suicide. I think the predecessor to that is mental imbalance. (SE)

In the above quotes, staff specifically connected spirituality to wellbeing. The above participant stated that spirituality could be accessed and promoted through cultural connection. This participant also noted how cultural connection and one's spirituality feeds back into wholistic development and moving toward a balanced life, ultimately promoting one's wellbeing. One staff member provided an example of the importance of being connected to one's culture to promote mental health and wellbeing, stating "when you are divorced from the land and divorced from your culture and your teachings and all those spaces, those are not going to be spaces of strength" (GR). This participant was speaking in historical terms; however, this concern is applicable in present day. Many Aboriginal people are not residing on their traditional lands, especially since over half (53\%) of Aboriginal people live in urban areas (Statistics Canada, 2006), and many may feel disconnected to their culture for various reasons (e.g., disconnection to the land). It has been noted that there are ways to connect to the land that don't necessarily require one go back to their traditional lands, which include going for a walk in a park or imagining that you are sitting on the land when residing in an apartment building or high 
rise building (J. Dallaire, personal communication, October, 2013). As well, RASS provides a space for Aboriginal students to connect and reconnect to their Aboriginal culture while living off-reserve and while pursuing their post-secondary education (Aboriginal Services, 2014).

Some staff members gave examples of traditional teachings that they use to promote their own mental health and wellbeing such as the teachings that we are all perfect beings (even in struggles with mental health), that the Creator is with us in our struggles, and that mental health struggle is a gift to be overcome:

I think we are all perfect beings. That's what the teachings have told me, that even in my struggle with mental health issues those are things I've put there because I am perfect enough to get through them. The Creator did not make mistakes in putting those there in front of me. [Mental health issue] is something I had to overcome and work through to be the person I am, so that I can be the perfect person (GR).

Because that whole idea that we are...We sat with the four great beings before we came here and put all of our struggles in front of ourselves so we could learn to overcome them is part of our perfection. That's one of the things then, that we are perfect in our struggles (GR).

The above quotes provide examples of how this participant has used her cultural connection to promote her wellbeing and cope with her struggles. The teachings she described allow her to apply an Aboriginal perspective to her mental health struggles, and she can use these teachings and this cultural connection whenever she needs them/it. Specifically, she has learned that she has entered the world exactly how she is supposed to (i.e., perfectly) and is supposed to go through her struggles, which is merely part of her perfection. This again, suggests a direct link between cultural connection and wellbeing, specifically, that such a connection can promote wellbeing.

Clash of Aboriginal and Western worldviews. Participants discussed a clash between Aboriginal and Western worldviews, especially with regard to mental health, as it was difficult for the two perspectives to be integrated. Students and staff discussed the differences and conflict 
between Western and Aboriginal perspectives on mental health as well as common misunderstandings between the two. More specifically, students drew attention to how traditionally, Aboriginal people looked after those in their communities with mental health problems in comparison to the Western way, which was perceived by students as disowning those with mental health problems, giving them medications for a "quick fix", or locking them away in mental health institutions.

The way they are, it's [mental health issues] part of who they are and uh based from our history there were mental health issues back then too, but the community back then accepted people as they were and just looked after them as just part of the community. They didn't just disown them or anything like that $(\mathrm{NH})$.

.... and they're [Aboriginal people] not just thinking, what can we do to fix that? And whatever it is. They know it [mental health issues] can't be medicated. It's going to take time and a lot of talking and more traditional medicines and how to use them to calm you down or relieve stress or learning how you can cope on a day-to-day basis. It's not just medicating you to be a zombie and shut-off (TL).

These two participants reference "disowning" people with mental health issues and "fixing" mental health concerns with regard to the Western way, which was a common theme discussed by many participants. These themes highlight key differences between Aboriginal and non-Aboriginal ways of healing as perceived by Aboriginal students. The first quote is in line with another theme that has been referenced above: that mental health is a part of the whole person (i.e., "it's part of who they are"). The second quote demonstrates another key difference between Aboriginal and non-Aboriginal ways of healing: time (i.e., "it's going to take time"). This is in line with literature that emphasizes relationship building in an Aboriginal context and a different perception of time within Aboriginal contexts (i.e., time is circular rather than linear; Christie \& Halpern, 1990; Smylie et al., 2003). 
Students reported that their Aboriginal culture is not often considered or acknowledged in the mainstream mental health system and that this system simply does not understand, respect or validate the Aboriginal perspective:

I guess where my concern would be like when dealing with Aboriginal people is that our culture wouldn't be taken into consideration when like assessing us. So it's like, for instance if you were to go and see like a psychiatrist or a psychologist, it's like because of their lack of maybe education within our culture they may not be as able to assess us as appropriately, right? $(\mathrm{NH})$

The above quote draws attention to the potential inaccuracies of the Western system in assessing Aboriginal people from a Western perspective, since often helpers within the mainstream system do not have an understanding of Aboriginal ways and how mental health issues may manifest differently in Aboriginal contexts.

Students specifically discussed the "disconnect" between Aboriginal and Western views on mental health: “They [Native people] do more spiritual stuff eh? And white people don't understand, so they don't let them come into the (inaudible)" (RT), "Ya like, if somebody wants to go for a sweat you know and um the Western society won't understand it and they say 'well, what do you want to go for a sweat for?' and they won't understand it and so they have to learn the...Indigenous way too" (KB). Both quotes speak to the mainstream system's misunderstanding or total lack of understanding of the spiritual component of healing, which is largely emphasized in Aboriginal contexts. The former quote speaks directly to non-Aboriginal people, more specifically, "white people" not understanding spirituality and the latter quote speaks directly to spiritual ceremonies that Aboriginal people use for healing, that would not be understood in Western contexts. The latter quote also calls for the Western system to make an effort to learn Aboriginal ways, in addition to their mainstream ways of healing. 
Students described how relationship building and trust is very important when working with mental health issues in an Aboriginal context and that trust and relationship building was perceived as being less important within the Western system by students:

...The Western point of view is where you're talking about the person...It's very closed off, it's very shut off, it's like them versus you, where they are analyzing you. As opposed to traditional counselling where they're actually trying to build a relationship and interact with you so that they can help further... What am I trying to say...They help you further cope with what you are dealing with, right? I think a lot of it is trying to build that relationship and that trust (DN).

This quote explains that, when one accesses treatment within the Western system, it is often one-sided, meaning that the helper is analyzing the client and the client is often the only one who is sharing his/her story. This participant compared the one-sided therapeutic process within a Western system to the Aboriginal way, which was described as involving work to build a relationship and trust and can move the helper-patient dyad to a more equal dynamic, thereby more effectively facilitating a healing process for an Aboriginal person. This is further described below:

From the Aboriginal perspective with more traditional counselling the counsellor shares a little bit of their story or experiences and knowledge with the client or whomever they're speaking with, and I'd like to say they are real with them right? They're not just sitting there jotting down notes and saying, what do you want to get done? Or, what do you think is a good idea? Do you know what I mean? $(\mathrm{NH})$

This quote further explains how a relationship between the helper and client is facilitated.

The quote explains that traditional counsellors share their stories with their clients and offer more self-disclosure (i.e., "they [traditional counsellors] are real with them [clients]"), which is a method of building trust through creating reciprocal relationships in Aboriginal contexts. This is compared to someone who merely reflects the client's questions and concerns back to them, while keeping who they are from their clients, further promoting a one-sided therapeutic dynamic and is not in line with Aboriginal culture. These ideas are consistent with a personal 
communication with J. Dallaire (September, 2013), who noted that Aboriginal people seek helpers who have "walked in their shoes", meaning that Aboriginal people who have suffered an addiction, often want a helper who has walked that path and has come out the other side with helpful coping strategies to pass on to their clients. Therefore, Aboriginal clients often want to hear about the struggle that their helper has gone through to learn about their healing journey (J. Dallaire, personal communication, September, 2013).

Similarly to students, staff noted that it is contrary to Aboriginal ways to take those with mental health issues out of their community or to institutionalize people. Rather, Aboriginal people traditionally "walked beside" their community members with mental health problems and did not dismiss them with medications:

Ya, so how we do it our way: I was talking to a Native lady the other day...'I'll walk with you' and so we are walking and we come to the edge of a cliff and I know you are going to fall over. The Western approach is 'ok, here is some medication and we'll see ya' and they fall over. Our way is 'ok you are going down, but I am coming down with you because I have to bring you back up' and that's how it works, you see? (SE)

The above quote provides a story that highlighted the differences between Western and Aboriginal perspectives on how mental health should be dealt with. There was agreement among participants that medication is sometimes needed for mental health concerns; however, a common theme was that medications should not be used to just 'fix' the problem and was perceived as an act of dismissal. Participants discussed the wholistic nature of mental health issues and how there are many other elements of healing through which one must go (perhaps in addition to medication) before they can move closer to balance.

Similarly to students, staff spoke about how the Western system has not been sensitized to Aboriginal ways and, therefore, there is often a clash between Aboriginal and Western perspectives on mental health. The following quotes directly compared the difference between 
Aboriginal and Western perspectives on mental health: “...first when the Western system meets with them [Aboriginal people]... They can't express where they are coming from because usually that Western person has not been sensitized to some of our ways. You know? So that creates a barrier that trust factor" (SE). This quote adds to a previous student quote regarding the lack of trust within a Western system and provides insight regarding why there is a lack of perceived trust within a Western context: lack of knowledge on the part of the mainstream system regarding Aboriginal histories.

...the Western model is very individualistic where you're just looking at one person whereas in terms of that [Aboriginal context] you definitely are an individual but you belong, you belong to a family, and you belong to an extended family, and a clan, you belong to a house, you belong to a nation, you belong in creation. (GR)

This quote speaks to the individualistic nature of the Western way in contrast to the collectivist nature of the Aboriginal way and recognizes the self as being relational, which is acknowledged in an Aboriginal context, but often not in a Western context. Such a relational concept is also connected to the idea that traditionally, Aboriginal people did not cope with mental health issues in isolation, rather they dealt with such issues in their family, community, nation, and creation, and further adds the idea of "walking with" or supporting people who have mental health concerns. Participants perceived the relational idea of mental health as being in sharp contrast to, and in some instances clashing with, Western ways. Findings from McShane et al. (2009), which examined Inuit parenting, found that the parent-child dyad could be conceptualized using both theories of autonomy and relatedness (with more of an emphasis on relatedness), which may explain why individualistic and collectivism are both emphasized throughout these results. It can be argued that Aboriginal populations are neither solely individualistic or collectivist, but rather, emphasize both on a spectrum. As well, Kagitcibasi (2005) has suggested that, with increasing urbanization, there is more focus on autonomy and, 
although Kagitcibasi (2005) did not in reference any Aboriginal group in Canada, it may provide some insight regarding a common individual (in addition to collectivist) emphasis among the student and staff population included in the present study.

It should be noted that, through the process of interpreting this theme, in particular, as well as interpreting all data, the principle investigator felt the clash between Aboriginal and mainstream perspectives within herself and worked hard to integrate the two perspectives that surfaced often from participants as well as from the principle investigator's own worldview, while emphasizing a focus on Aboriginal worldviews, as was the aim of the study. The struggle for integration as well as the common shift from Aboriginal to mainstream perspectives within the principle investigator was initially seen as a problem to be solved and, throughout the study, this viewpoint as shifted to an acknowledged and accepted way of interacting with the project as well as the world, especially since this clash, the common shift between Aboriginal and mainstream worldviews, as well as the struggle for integration between the two is an ongoing process, reflecting not only the principle investigator's daily process, but also the process of many of the participants in the current study. This process represents the common call for individual, communal and systemic changes regarding cultural revitalization and acknowledgment in order to make this process smoother, less distressing and less confusing for Aboriginal people.

\section{Summary of mental health from an Aboriginal perspective as it informs the KT}

Phase. According to Aboriginal students and staff, mental health was considered to be only one dimension of the whole person, a community responsibility, and having been passed on intergenerationally. Mental health was also viewed by Aboriginal students and staff as greatly impacted by the following: identity confusion, denial, and pride; negative stigma (especially as 
related to accessing treatment); and one's connection, reconnection, or disconnection to their Aboriginal culture. Aboriginal students and staff also identified some important clashes between Aboriginal and Western perspectives on mental health, especially regarding the emphasis on spirituality in Aboriginal contexts and in the way that mental health is managed within each context. See Figure 1 for a visual representation of the definition of mental health from an Aboriginal worldview.

The above definition of mental health was emphasized throughout the Knowledge Translation Phase of this study. As well, from a recommendation by staff members, the term "mental health" was not used often during the activity due to the stigma with which this term is associated. To acknowledge the wholistic nature of the mental health concept, the term "wellbeing" was instead used throughout the Knowledge Translation Phase.

It should be noted that, although each of the above themes were separated by a subheading, the intention was not to suggest that these themes were entirely distinct. It is important to acknowledge that these themes were interconnected and interacted with one another to create an understanding of mental health and wellbeing from an Aboriginal worldview. Therefore, some of the content of the themes discussed will overlap with one another and this is acknowledged as an inevitability. For example, quotes constructing mental health as a wholistic concept, impacted by identity, and affected by cultural connection were found to overlap in various ways, but were kept under separate headings due to important qualitative differences found between these three themes.

\section{Knowledge Translation Activity Content}

Coping with mental health concerns. Participants spoke about how Aboriginal students cope with mental health problems to improve their wellbeing. Such coping strategies were 
separated into individual coping strategies, communal coping strategies, and coping strategies that could be either individual or communal.

Individual coping strategies. Individual coping strategies includes anything that participants discussed that are often done alone/without the influence of other people. Student participants mentioned the following individual coping strategies: reading about whatever they may be going through (e.g., looking up their concerns online); watching TV; sleeping; writing in a journal; praying; meditating; having a hot bath, shower, or sauna; reading a book; crying; getting a massage; listening to music; turning to traditional medicines; praying to the Creator; and smudging. It was also suggested by students that people go to the Emergency Room or admit themselves into an inpatient unit if there is concern that they may harm themselves.

The following quotes demonstrate individual coping strategies that students may use: "I also do turn to my medicines. I like sage, tobacco, sweet grass, cedar. So sometimes I'll also make like a cedar tea...I have bought this special medicine. I had it made by an Elder. It's supposed to help me with stress so I should be doing that" (DN), and “...I think saying pray is funny. When I say pray, I think Christianity. So I don’t like to say I pray to the Creator, I like to say that I give thanks. I ask that he guides me, and he will help me and puts things and people in my path to make sure I'm going to be okay" (DN). These quotes were from a participant who had previously spoken about dealing with mental health on her own. These quotes also demonstrated how she might use her cultural connection as a coping strategy and the examples she gave are usually implemented alone. The number of individual coping strategies, as mentioned by participants, is surprising since traditionally, Aboriginal culture is very community-based; however, many youth are only beginning to reclaim their Aboriginal identity and, therefore, the types of coping strategies they are currently using may reflect the historical 
trauma, and internalization of Western individualized constructs of 'coping' that have been passed on intergenerationally from their ancestors through colonization.

Communal healing. Communal healing includes any discussion of healing through involving others/influence from others. Students mentioned coping strategies that are often done with other people to promote healing, such as talking to family and friends for support, socializing with others, talking to an Elder, going to a sweat lodge ceremony, and dealing with mental health issues within the family. One participant specifically spoke about the importance of having friends in your life to help you cope with mental health stressors, stating:

Talk to friends because at the end of the day you're lucky if you have your parents, but like your circle of friends is also very important in your life, so if they're in the know and they know what's going on with you, so if you're acting a little weird and they notice, then they can do their best to help you out. (TL)

The mention of coping strategies that are often done within one's family, social network or community, is in line with traditional Aboriginal ways. Traditionally, wellbeing was promoted through interactions with other people, rather than in isolation. Participants in this project have discussed the isolating nature of mental health as a "Western way" rather than a Aboriginal way.

Staff members spoke about involving the whole family when someone is mentally ill: “...there is an Aboriginal Elder and healer who can work on schizophrenic people and put them back in the right place, but it takes the whole family to do that. There is one man who can work with that, but he won't do it unless the whole family is involved, ok?" (SE). This quote specifically draws attention to the importance of getting the family involved in one's mental health concerns and how the particular Aboriginal healer this participant was referencing will not work with an individual without the commitment of the family. As well, staff mentioned that different caregivers should be brought together to address all dimensions of the self: "For me, I have a person that I talk to about my dreams, I have a person that I talk to about my feelings, I 
have a person who reminds me about my health, and I tend to my spiritual self. If I don't have all of that, then everyday becomes more difficult" (MB). This quote is in line with the above theme related to the wholistic nature of mental health and how different people need to be brought together to attend to the whole self.

Individual or communal healing. This theme includes any healing strategies discussed that could be either done alone/without influence from others or in the presence of others/with influence from others. The following are coping strategies that were suggested by students and could be done either alone or with others, as it was unclear for each strategy whether students would use them on their own or with others: going for a walk, going to the gym, laughing, making music, going on nature hikes where "your Native comes out", making drums, drumming, singing traditional songs, beading, connecting to the land (e.g., by going to a park or going back one's Aboriginal community), and turning to your ceremonies. Many of the traditional coping strategies such as drumming, beading, singing traditional songs and turning to ceremonies were traditionally done with others; however, this assumption cannot be made due to the historical loss of Aboriginal culture and the forced assimilation with which many Aboriginal people and their ancestors have faced. Therefore, it is possible that these activities are being done in isolation. On the other hand, youth and other Aboriginal people are reclaiming their Aboriginal identities, traditions and cultures and, therefore, these strategies could be done with one's community members.

The following quote provides an example of a coping strategy that could be done alone or with others: "I also more recently have been trying to be more traditional and learned beading and drumming as well as like singing. I recently made a drum, and yesterday I was feeling really stressed, so I drummed and I sang and I felt so much better after that” (DN). Regardless of 
whether the coping strategies mentioned by this participant were done individually or with others, such cultural connection seemed to increase her own sense of wellbeing.

Potentially unhelpful healing methods. This theme captures healing methods that were identified by participants as unhelpful, especially if used in access. Students also spoke about coping strategies that may have the potential to be unhelpful if used too much (i.e., they could be helpful if used only in moderation). Such suggested coping strategies included having sex, using substances, watching TV, sleeping, beating something up, eating carbohydrates, and making a joke out of mental health issues. These coping strategies may be helpful until one becomes dependent on them or until they are being used for maladaptive reasons (J. Dallaire, personal communication, October, 2013).

Summary of healing strategies as it informs the KT Phase. The coping strategies listed above as mentioned by Aboriginal students and staff were communicated to Aboriginal students during the Knowledge Translation Phase of the study and students were given a handout within a larger booklet of resources with a list of possible coping ideas. Finally, other coping strategies were added from presenters/facilitators of the activity. See Appendix U for Wellbeing Activity Booklet that was given to all participants.

Mental Health Resources for Aboriginal students. This theme relates to any discussion of resources or services that Aboriginal students could access in regard to a mental health concern. Students listed organizations where they have or would access services if they needed help for a mental health issue. Students suggested talking to someone at RASS and that people at RASS can refer students to specific services depending on the issue: "Mmmm hmmm. Last semester I actually had a bit of a breakdown. I was just so stressed out (inaudible) she [staff member at RASS] said 'you're going to see [other staff member at RASS]'” (TL). This quote 
demonstrates that RASS staff members have a role in advocating for students to help them access what they need. Students spoke about how RASS has traditional counsellors and women's circles to help connect students with their culture. Students noted that Anishnawbe Health in Toronto, Ontario is a resource that could be helpful, but that there were some issues students have experienced that may make this resource difficult to access (i.e., you need an Ontario Health Insurance Plan; OHIP and you can't have a family physician already). It was also suggested to go to your family physician, but one participant in particular stated that she would not go her family physician for a mental health concern because her family physician was "too busy". Students spoke about Native Child and Family Services in Toronto, Ontario, but were unsure how well this service would work for individuals who have children:

Native Child, I mean I know there is that stigma around it, with them being up in everyone's business with their children, but for people who don't have that kind of connection with them like myself, right like I can go in there and they have counsellors. They have like addictions counsellors and like they're good. But you know, you might not want to go and see a counsellor there if they are like taking your kids, right? So a lot of people don't go there. It is what it is. It's a good place (NH).

This quote demonstrates the intersection of available resources that Aboriginal students may know about and the stigma around accessing such services, which may limit access depending on one's circumstance. Also, the participant suggested that Aboriginal people could feel the need to avoid interaction with any sort of child welfare agency to prevent a repetition of historical trauma, since child welfare was a powerful means through which the Aboriginal population in Canada was traumatized: through the Sixties Scoop, for example. Once residential schools began to be phased out, the government decided that there needed to be other ways to "protect" Aboriginal children from their culture (Aboriginal Healing Foundation, 2007). In the 1960's Aboriginal children were apprehended from their families and placed in child welfare agencies or adopted by non-Aboriginal families. The Sixties Scoop caused further disruption in 
Aboriginal culture and a sense of identity confusion for many Aboriginal children and their communities (Aboriginal Healing Foundation, 2007).

Students spoke about accessing treatment through a psychologist or psychiatrist and one participant in particular expressed her disappointment with the mental health system, as it was difficult for her to find a counsellor with whom she could connect:

I've been really disappointed by like health, I guess just my own experience personally just, I haven't been able to connect on or get the help I really needed from other people in different organizations. Either I didn't communicate my situation properly or I just didn't understand um, so I usually resort to people closest to me because they just know who I am. (HJ)

The above participant discusses the issue around feeling connected to her counsellor and highlighted the importance of the relationship, since without a relationship, students perceived services as being less helpful. The participant provided insight regarding the possible disconnect between this participant and her counsellor and the impact that such a disconnect had on her. This participant seemed to be resorting to self-blame for the system not working for her (e.g., “...I just didn't understand...” and “...I did not communicate my situation properly...”), which can translate into further struggles with one's wellbeing. Regarding the relationship issue, there was a suggestion by participants that people try many different counsellors before finding one with whom they will "click". The following quote demonstrated this idea:

... I just clicked with her [counsellor]...I don't need to see her anymore, right? It's like I go and see her once a month. We're basically friends now it's like we just clicked that right away, you know? But it's like I went and saw another 10 [counsellors] before her that I just didn't click with. Sometimes it's just that, right? You just got to come and it's like looking for a job, you know? (laughs) $(\mathrm{NH})$

This participant notes that "We're basically friends now..." and, therefore, "clicking" with someone may be enabled by creating an equal dynamic between the client and counsellor. This is also in line with Aboriginal culture since hierarchy and power dynamics is something that is often not emphasized, as it is within a Western context (Castellano, 2004; Smylie, 2003). This 
quote may also tap into the idea that accessing mainstream services can leave Aboriginal people feeling misunderstood because there is often a lack of knowledge regarding the historical context from which Aboriginal people are coming. Such a misunderstanding can limit access to available resources or necessitate much time devoted to finding the "right" helper.

Other resource suggestions included calling Kid's Helpline, going to the ACCESS Centre at Ryerson University, going to a Naturopath, accessing services through the Centre for Addiction and Mental Health, going to an Elder, accessing a Healing and Wellness worker at a Friendship Centre, going to a traditional healer and connecting with an Assertive Community Treatment team for individuals with severe mental illnesses (e.g., schizophrenia).

Staff primarily discussed RASS as a helpful resource for Aboriginal students. Staff explained that their healing model is about putting the person who is struggling, in the middle of the circle (i.e., have staff at RASS band around the student in support), so that students know they are supported. RASS also works to create trust with students and build relationships. Another staff member mentioned Anishnawbe Health as a good resource for those with mental health issues. The following is an example of the service that RASS offers from a RASS staff member:

That's that model again of putting the person in the middle. There's only so much you can bring to a person, it's the confidence. I want to bring up the topic of being confidential, with this particular model you can be sure that what you tell me I will keep in confidence. However when I start to feel there are some red flags going up, I'm probably going to tell you at some point that I'm going to go talk to these other folks who you already know about, and when you're ready we're going to sit with you...(GR)

This explanation demonstrates the process of putting the person in the middle of the metaphorical and, at times literal, circle and how this may necessitate letting others know the struggle this student may be going through so that students can be helped in the best and most wholistic way possible. This idea also interacts with a communal way of coping with mental 
health issues and promoting wellbeing. RASS is considered to be a community organization and, therefore, this model of helping is very much in line with the Aboriginal perspective of wellbeing and promoting wellbeing.

Summary of resources as it informs the KT Phase. Resources that students suggested incorporated both mainstream and culturally safe services. The resources listed above as well as anything that students noted when accessing such services (e.g., you need OHIP to access Anishnawbe Health) was incorporated into a resource handout, so that Aboriginal students had a comprehensive list of mental health resources. As well, the research team conducted a thorough search of other mental health resources in the Greater Toronto Area that students from Ryerson University can access. See Appendix U for the Wellbeing Activity Booklet that was given to all participants and includes a resource list.

Gaps in mental health knowledge. Students spoke about mental health information that they would like to know more about. Such knowledge fit into two themes: General mental health knowledge and knowledge about the mental health system.

General mental health knowledge gaps. This theme includes discussion of anything that students wanted to know more about in relation to mental health/wellbeing other than the mental health system, specifically. Participants were interested in increasing their understanding of their emotions, which was identified as a theme through the following discussion regarding why certain activities don't make people feel better or positive emotions are so difficult to maintain when one is feeling down: "Ya, because even then it [a certain activity] doesn't feel good, the stuff that you are like doing. It just feels like you're going through the motions" (TL), "Or it's [the positive emotions] fleeting, you know? Because it's like you will do it for a while..." (NH), "Or it's like, right it will be like oh it [the good feeling] was there for like 10 minutes and then it 
just like went away and now I don't feel like doing it [an activity] again" (TL). Here, students discuss how difficult it is to cope with negative emotions and how they might attempt to engage in activities to make themselves feel better, but it is difficult to commit to such activities when their emotional responses are not changing significantly or right away. Such a discussion indicates that students may benefit from some information on behavioural activation (Martell, Dimidjian, Herman-Dunn, 2010).

Student participants also wondered why people sabotage themselves through selfdefeating behaviours and tear themselves down: "I just I don't know why, for some reason mental health, like when you're experiencing a bad time, you sabotage your own self and for some reason you don't get the help you need because of pride or I just don't understand how that makes any sense" (TL). This participant describes a cycle of unhelpful thought and behaviour patterns, which she expressed as confusing to her, since such patterns did not promote wellbeing, rather it contributed to struggle. Her confusion fits within the historical context of Aboriginal people, who are living in a mainstream context. Throughout generations Aboriginal people have been forced to adopt a European way, which is often individualistic and in contrast to traditional ways of knowing and doing. The perplexity expressed above seems to represent a clash between Aboriginal and Western worldviews, especially with regard to perspectives on mental health.

Students wanted to know why people want to avoid social situations when they are really upset: "Ya, like why is it like when you're feeling really upset that you don't want to like be cuddled by your friends? Why is it that you want to separate yourself from them?" (TL). This participant indicates that it would make more sense for someone to want to be soothed when they are upset, since this would contribute to more positive feelings, but found it confusing that this is not often people's first instinct when struggling with difficult emotions. This quote may parallel 
discussions of cultural revitalization within many Aboriginal communities, as the Western way is to cope in isolation and the Aboriginal way is to cope with one's community. This participant may have demonstrated a need to move closer to her traditional ways of coping (i.e., through her community).

There was a brief reference to how to challenge the stigma related to mental health, "Ya, like how to challenge it [stigma]? Ya, that's a cool question" (NH). There was little discussion about this topic in particular, which may indicate that stigma may be seen by participants as too big or too difficult to challenge and such a lack of discussion may indicate fear or a sense of helplessness or hopelessness regarding whether it would in fact be possible to challenge mental health stigma.

Students expressed curiosity about what life situations may be behind addictions and anxiety:

Ya, like what's behind it because they have a psychologist for like hoarding and what not or My Strange Addiction or Intervention, like there's always someone there, but they never really dug deep and obviously it could be due to confidentiality for some aspects, but that's all that like we see from a Western point of view (TL).

The above quote indicates that students want to hear the story behind someone's struggle, which aligns with Aboriginal ways (J. Dallaire, personal communication, October, 2013). Often, healing is done through story telling (i.e., either hearing someone's story or telling one's own story; J. Dallaire, personal communication, October, 2013) and, therefore, it makes sense that Aboriginal students may express curiosity regarding how someone may have developed a mental health issue such as an addiction or anxiety disorder.

One student in particular wondered about the different medications for mental health issues, the patient-helper dynamic and the mental health system in general, "Yeah actually, I would like to know more about mental health all together, like why do they have certain meds, 
what about the patient relationship, you know?..." (DN). This participant described some mental health issues within her own family and because of this, she had some encounters with the mental health system. Therefore, she had many curiosities about mental health issues that affected her family.

A student participant asked about the different degrees of various mental health issues and how to use different coping strategies to help (especially for depression and anxiety):

I would like to know more about mental health in general. So like schizophrenia, the different degrees of it, how it affects the person and how to deal with that person, or the different degrees of depression. Also, anxiety and how to deal with that or different ways to cope with all of these things. That's just because I like to learn a lot (DN).

This same participant noted, "We all experience anxiety or depression at some point. It doesn't mean it's at a clinical level, but we all experience it. So, to know how to cope with it and stop it from getting worse" (DN). This participant was aware that mental health issues manifest differently for different people and have different severities, but wanted to understand more regarding how the different degrees of mental health issues may manifest. She also drew attention to an important concept with which other participants agreed during the discussion: How to cope. Coping was not talked about with regard to getting rid of mental health issues, but rather regarding how to manage difficult emotions, reactions, and situations when they arise.

Another participant wondered: "And also, what about the families? How can they cope with a person who has mental illness?" (LV). This participant is positioning an idea that mental health issues can be dealt within the family system, especially in Aboriginal contexts, since coping with mental health in the family is a traditional way. Therefore, it is not surprising that a participant posed a question related to mental health issues within the family.

Related to coping with mental health in the family, students wondered about the appropriate terminology to use when talking about mental health in order to support their friends 
and family, who may be suffering,

...but just like the terminology, you know if I meet someone, how do I interact with them and not...? Because it can be a sensitive thing for them and I don't want to be like saying the wrong thing on their issues. So I guess that's the first things that comes up when you say 'mental health' is just how to interact with people who may have those kinds of issues. (LV)

This participant was trying to be sensitive to mental health issues with which others struggle and wanted to be able to use terminology that would not be stigmatizing or offensive to others. This relates to the topic of coping with others, as one way to do this could be through using appropriate terminology to support and be sensitive to those who may be struggling. This is also in line with the communal perspective on mental health, as gaining knowledge on appropriate terminology can ensure that you are able to support people who may be struggling in one's community.

Related to supporting families and using appropriate terminology among student participants, was how to be supportive of someone you know who has a mental health issue while taking the ethic-of-noninterference into consideration. The ethic-of-noninterference refers to a communication style that is devoid of argument and advice giving, promoting a nonintrusive model (Castellano, 2004). "Just like how to be supportive, just like more supports. For me just my roommate wanted to quit smoking and I kind of had the old school like 'just quit', 'don't be like, you know, just be strong. It's easy"' (LV). This quote refers to wanting to understand what kind of language to use to help support someone who may be struggling and hoping to move away from using language that is invalidating for people. The discussion of supporting others is further demonstrated below:

Going along with being supportive like helping them make connections and not being afraid, I guess because like since there is so much stigma, a lot of people don't want to seek that treatment but it's like you know you want to help them to try and do something to help them, right? (WN) 
This quote draws attention to supporting significant others in accessing treatment, despite the stigma that surrounds that decision. This participant also noted the fear that results from stigma and how this interferes with accessing treatment. This participant positions herself as helpless in the face of a mental illness at times, since stigma and fear create barriers to her ability to help and support the people in her life that might be struggling. She is requesting methods for how to overcome these obstacles. A similar struggle is demonstrated below:

Well, more things I would want to know about are like more intensive um support I guess, so like people who are on prescriptions and they are also self-medicating so what can you do? Like there is nothing you can do because they are an adult and it's their choice basically at that point. (WN)

Ya, because we were talking about how in an Aboriginal perspective you're supposed to, it's this is their journey and this is how it's supposed to happen I guess, but at the same time you're their family like what are you going to do? (WN)

The two quotes above begin to tap into the consideration of the ethic of non-interference (e.g., "...there is nothing you can do because they are an adult and it's their choice..." and “...it's their journey and this is how it's supposed to happen I guess..."). These two quotes raise the question of how to balance the support of one's family and friends, who may be struggling, with a traditional way of not interfering with someone's life path.

Systemic knowledge gaps. This theme includes discussion of things that students wanted to know more about that were specifically related to the mental health system. Students spoke about wanting to understand how they can know whether a service will be helpful and confidential, more services where they could get help for a mental health concern, how to access treatment, different types of treatments, how to promote cultural awareness (e.g., using a wholistic way of healing), and what questions to ask when accessing the mental health system.

...we kind of talked about making connections, but how to like seek out treatment or support... and also what to look for, you know? Because it's one thing to just say go to your doctor, go to the nearest therapist, you know, but what if that's a bad therapist? What's good treatment? What are the different types of treatment out there? (DN) 
This quote draws attention to the lack of awareness that people have about the mental health system with respect to available options and knowing when to change direction with your treatment plan. This participant expressed curiosity about how to get to a place where there is confidence in what you are doing and whether or not you are getting the best treatment. This participant spoke specifically about making connections and refers to "good" treatment and "bad" therapists. She is likely positioning good treatment as that of fostering relationships. The quote below also demonstrates participants' curiosity about the mental health system:

How does that system work? I think about my [family member] a lot who deals with that person who controls the money but doesn't work with this person who's supposed to work with this other person and no one is communicating. So, he has all these people who are supposed to be helping him but if they aren't communicating how are they supposed to get anything done? You know what I mean? (DN)

This quote demonstrates general curiosity regarding how the system works and why it functions in the way that it does (i.e., with a lack of communication between services and very disorganized). This participant wants to support her family members who are struggling with mental health concerns while being situated in a dominant and mainstream context that silos various area of healthcare. She expresses uncertainty regarding how to do this.

One student also wondered how Aboriginal patients might be dealt with differently within the system (e.g., with regard to Indian Affairs), "Yeah, the whole system. Especially how patients are dealt with when they are Aboriginal because it is different" (DN).

Like, let's not lie here, Aboriginal people are dealt with differently because we have Indian Affairs and we have coverage underneath that and so, they deal with us on a whole other level. That goes back to the meds. Because we are covered we don't get the top stuff we are supposed to, we get generics (DN).

There is an assumption here that Aboriginal people are treated differently in the mental health and healthcare systems and a specific example of medication access was provided. Although not explicitly stated in this statement, there is an undertone referencing stigma, 
stereotypes, and racism toward ethnic minority groups that exists within the mental health and healthcare systems, which can impact how Aboriginal people are dealt with in these systems and can be an additional barrier to benefitting from the system. This idea is demonstrated through the example of medication access.

Summary of knowledge gaps as it informs the KT phase. Aboriginal students wanted to know facts about different mental health issues, how to cope with various mental health concerns, medication information, and how to be supportive of others with mental health issues. As well, Aboriginal students wanted to understand the process of receiving help for a mental health issue, including questions to ask and treatment options. Such questions posed by Aboriginal students were addressed during the Knowledge Translation Phase of the study. See Appendix U for Wellbeing Activity Booklet that was given to all participants and includes a Medication Information: Questions to Ask handout.

How to get mental health information. Students spoke about ideas regarding where and how they could get mental health information to address some of the gaps in their mental health knowledge that they identified. Such suggestions related to three different themes: (1) information from services, (2) information from people, and (3) information from the Internet. Staff did not speak to this topic because time did not permit such a discussion.

Information from services. This theme includes participant's discussions about services through which they could gather mental health information. Regarding obtaining mental health information from services, students suggested the following: Help lines, Anishnawbe Health, pamphlets (e.g., how to help someone quit smoking), the Centre for Addiction and Mental Health, Schizophrenia Association, Friendship Centres, Mothers Against Drunk Driving, school counsellors, RASS, the University of Toronto Aboriginal Centre, The Native Canadian Resource 
Centre, and Chiefs of Ontario. "I think that's a good resource: The Native Canadian Resource Centre. I don't think we said that before. Is that what it's called up on Spadina there?...That's a good resource. It's like a friendship centre they've got all kinds of resources up there" (LV).

You could also go to CAMH place and there is a lot of different organizations like CAMH that, not just here, but I'm sure there are Ontario organizations and non-profit organizations that deal with I'm sure...the key is if they have mental health in their title then that is probably a good place to go $(\mathrm{LV})$.

This participant suggests that people visit these resources to gather mental health information and expand upon their understanding of themselves.

Information from people. This theme includes participants' discussions about people through which they could gather mental health information. Regarding mental health information from people, students suggested friends, family, social workers, traditional counsellors, psychologists, going to a lot of different people that you trust with a question and then synthesize all the information for yourself, asking people who have gone through whatever you are wondering about, and talking to other Aboriginal people. One student said, "I might ask a social worker or a traditional counselor or psychologist or someone I know where I could get more information..." (DN). This quote demonstrates some trust in the healthcare system, as this participant acknowledges that these professionals can be trusted to provide accurate mental health information. Another participant suggested people ask, "Any just, Aboriginal people, just people who have gone through it..." (BG). This participant draws attention to the experiential knowledge that is emphasized and highly valued within Aboriginal contexts (Jack et al., 2010).

Information from the Internet. This theme includes participants' discussions about Internet websites through which they could gather mental health information. Regarding mental health information from the Internet, students suggested that people "Google" whatever it is they want to know about, visit chat rooms such as supportgroups.com. Regarding supportgroups.com 
in particular, students noted that it was easy to create an account and that it has different chat

room forums for a variety of mental health issues.

What I liked about is, that it [supportgroups.com] is easy to make an account there and then they have like um, they have like probably I would say close to 100 like different they have groups like different chat forums like active to, like people are on them that like people you could have like addiction. Any kind of addiction you could think of. Not just like your typical alcohol, cocaine, heroin, like all the other like random ones on there. As well as like depression or like OCD. Just like anything and everything, right? I thought that was like that's a cool site because like people go on there and they have like those issues as well and have often went out and sought help like in the medical field profession as well and then it's like kind of talk about those things together (TL).

This is an example of Aboriginal students seeking support by creating a sense of community for themselves. The description of supportgroups.com above demonstrates the idea that, although Aboriginal students use individualistic coping strategies as described above, they also seek out community-based strategies, which shows the resiliency of this culture, as both individualistic and collectivism was emphasized traditionally (McShane et al., 2009).

Students suggested that people visit the CAMH website and a website entitled "Six Billion Secrets", which is where people provide chronicles of what they are going through and students spoke about this website as being very supportive and it is in line with the storytelling tradition of an Aboriginal context:

...chronicles what people are going through. It's call Six Billion Secrets and like a lot of these people post it anonymously or they won't put their real names but their location will be there so like there is like a sense of anonymity but like people don't put the other person down if like something terrible happens to them. They feel upset. On the contrary, they try to help them, even though they don't have like a name to the face or anything (TL).

The following quote is another example of how one student finds mental health information on the Internet: “... and then I would Google away, I would Google stuff and wonder if it was true" (DN). This last quote more explicitly demonstrated that students tend to have uncertainties about what they are accessing on the Internet and whether the resources they find 
are in fact credible. These quotes alluded to the fact that students have looked for resources on the Internet and have found some that might be helpful to them.

\section{Summary of how Aboriginal students get mental health information as it informs the}

KT phase. Aboriginal students tend to obtain mental health information from services, people, and the Internet. The above suggestions of how and where to get mental health information were communicated to Aboriginal students as well as other suggestions from the facilitator/presenters during the knowledge translation activity.

\section{Knowledge Translation Activity}

Students and RASS staff members were asked questions specifically about how to design a knowledge translation (KT) activity to most effectively communicate mental health information. Such responses related to three different themes: (1) the KT method, (2) the KT content, and (3) the KT presenter or facilitator.

Method. This theme includes participants' discussions of the way in which the KT activity should be implemented. Student's noted that the activity should not be lecture-style, as they considered this style to be "boring"/not engaging or conducive to learning. Rather, students suggested that the facilitator/presenter talk to the students, rather than at them, and that the activity be discussion-based, "Activity. If this was a lecture style I wouldn't be here (laughs)" $(\mathrm{NH})$ and "I just think like when we're doing this [focus group], it's like you're talking with us versus if like you're doing a lecture, you're talking to us" (TL). Students suggested that the activity be conducted with smaller groups of people and that storytelling be used to communicate information. Students suggested that the activity be done in a sharing circle so that everyone is equal and that it begin with an opening prayer and smudging. Students agreed that the Aboriginal way should not be compromised during the activity: 
...I know you're trying to be pragmatic and like always doing the theoretical, but it's like this is where like the crux of the problem like exists to me because like I think of what we are doing right now, like we are talking about the Aboriginal approach and like and then slash the Westernized approach, and maybe more appropriately 'versus' because I just don't know if they can um coexist because one has got to compromise the other to like be in tune with the other and it's like always like the Aboriginal one that like [gets compromised]. (NH)

The above participant expressed his frustration about the frequent breakdown of Aboriginal approaches within mainstream contexts due to the difficulty in bringing together an Aboriginal and Western worldview. As a result of these frustrations, he expressed his uncertainty regarding whether the activity can really stem from an Aboriginal approach, as it is common to leave out the Aboriginal way due to the pragmatics as well as the Western dominance in the system.

The following quote supports the idea that Aboriginal culture and traditions should be used to guide the activity: "Ya, I think like the way that you did this [the focus group], like it's really important that you did it [the focus group] in this way [opening prayer and smudging], you know and how it's just like you do that every time, you know? Just like with the smudging you know?" (NH). There was also a discussion about how students learn best in order to structure the activity in a way to support such learning, and there was agreement that students learn well both visually and orally. One participant in particular spoke about her learning style, "I learn best by talking about things and then hands on stuff. So dealing with things hands on. I think that's how most Aboriginal people are. Like you show them something and they're like okay I can get that going. That's how I learn anyways, hands on or visually" (WN). Therefore, through incorporating Aboriginal ceremonies and methods of learning that are conducive to Aboriginal people, the activity can be engaging and facilitate new learning.

Staff suggested that the activity be done in a forum and, if lots of people attend, the circle 
could be broken up into smaller circles. Staff also suggested that it be called a "Wellbeing Training" and that there should be a preliminary session and an ending session. As well, staff suggested that the focus be on a wholistic understanding of mental health and the fact that people may not be ready to learn some of the things that are covered during the activity should be understood. The following quote demonstrated how staff thought the activity should be implemented:

So a wellbeing training or even looking at it as a space of new knowledge as a way to engage people so they are not coming in with the expectation and the armor because 'mental health' creates armor for people. There is so much shame and blame that people come in with armor on and they may not be able to hear it and I really think that in giving a wholistic understanding that is going to remove the terrifying labels. It's about changing even the understanding that mental is the problem (GR).

This staff participant suggests that facilitators of the activity work to remove the stigma around mental health and to re-define the terms so that people can give up their "armor" and just have an open and honest discussion about the topic. The quote also highlight the wholistic emphasis that should occur throughout the activity.

Content. This theme includes participants' discussions of what material should be covered during the KT activity. In addition to a discussion about what mental health is, coping strategies, resources for mental health issues, gaps in mental health knowledge, and how to get mental health information, staff and students made some specific suggestions about what exactly to cover during the knowledge translation activity. Students suggested discussing coping strategies, how to stop the shaming and blaming as related to mental health, how to recognize mental health symptoms, terminology to use when talking about mental health, medicine wheel teachings, how to use traditional knowledge to maintain your mental health, and sharing personal struggles. The following is an example of something that one student in particular wanted to talk about during the knowledge translation activity: "I think just the connection between general 
traditional knowledge and using that knowledge to maintain or to improve your mental health"

(NH). This is an example of a participant who wants to use her cultural connection to increase her wellbeing.

Staff suggested talking about how mental health impacts everyone, suicide, the fact that it takes a community to deal with mental health issues just as it does to raise a child, the importance of going to ceremonies, and that Justice Sinclair's ${ }^{3}$ four questions be used during the activity, which would ask students to try to answer the following: Who am I? Where am I from? Where am I going? What am I here to do? This is demonstrated by the following quote:

Those questions come from different traditional teachings. We have three key ones and he added a fourth, which is current to where we are at this point in time as Aboriginal Indigenous people. Those are critical pieces because often you don't ask people to think about who they are and why they're here (GR).

The suggestions by both students and staff regarding the content of the KT activity also reflect the cultural connection theme, as both groups suggested using cultural connection during the KT activity to promote wellbeing.

Presenter/Facilitator. This theme includes participants' discussions of who exactly should implement the KT activity. Students suggested that two people facilitate the activity: (1) an Elder and (2) someone with the academic background:

Yeah, I think it should be an Elder in regards to whether the person is elderly or not. I think it should be someone who has knowledge in traditional counselling. It could be also someone who has a background in social work, or psychology, or mental health. So they have a greater understanding of the person's mental capacity... it should maybe be conducted with two people because more often than not an Elder doesn't have a background in social work or mental health or any of that but their traditional knowledge is going to be important...(DN)

This participant is acknowledging the fact that Elders can be young people; however, this

\footnotetext{
${ }^{3}$ Justice Sinclair is the Chair of the Truth and Reconciliation Commission of Canada and the Associate Chief Judge of the Provincial Court of Manitoba. He was also Manitoba's first Aboriginal Judge (Truth and Reconciliation Commission of Canada, n.d.).
} 
is a rarity and that the recommendation for having an Elder co-lead the activity is about traditional knowledge, rather than age. She noted that someone with a background in social work or psychology could supplement the knowledge offered by an Elder. Staff suggested that there should be seven people speak during the activity because seven is a sacred number. It was suggested by staff that such speakers be "high profile" such as Theo Fleury, who is Métis and a professional Hockey Player.

Summary of the KT strategy. The suggestions made by students and staff regarding the method, content and presenter/facilitators of the knowledge translation activity was used to design the activity. More specifically, the activity was implemented using: traditional ceremonies, emphasizing the Aboriginal way; using one activity to reduce attrition; and, the activity was facilitated by both an Elder and the principal investigator (CJD; who has training in psychology). The planned topics are outlined in Appendix V: Facilitator's Agenda of the Wellbeing Activity. Also, see Appendix U for the Wellbeing Activity Booklet that was provided to all participants of the activity, which includes the participant's version of the activity agenda. 


\section{Results: Knowledge Translation Phase}

This portion of the results is separated into three different sections: (1) The Activity Evaluation, where participants' written responses to questions about their mental health knowledge and self-efficacy, asked before and after the Wellbeing Activity, are presented. Their responses to requested feedback on the helpfulness of the activity, collected immediately after the activity, are also presented in this section. (2) Knowledge to Action, where participants' responses about their knowledge retention and how they are using the knowledge they learned from the activity in their daily lives, collected during the one-month follow-up interview, are presented. (3) Global Messages, Current Challenges, and Ideas for the Future, where participants' responses on more global and overarching questions asked during the one-month follow-up interview are presented.

The results from this phase are presented through a description of metathemes as well as themes found to fall under each metatheme. Not all participants spoke to each theme. It should be noted that initials have been placed after each quote to reference each participant. In order to protect participant confidentiality the initials used are not those of each participant's name. Participants in this portion of the results all identified as Aboriginal post-secondary students.

\section{Activity Evaluation}

Four Aboriginal students attended the Wellbeing Activity. Participant descriptive information is presented in Table 1. Participants were asked questions about their mental health knowledge and self-efficacy prior to and post activity. See Table 2 for descriptive information on these scores. Participants were also asked, immediately after the activity, what they found to be acceptable and what they would change about the activity if it were to ever be implemented again. 
Acceptable components of the activity. This metatheme captures discussions of aspects of the activity that were acceptable to participants who attended. Participants were asked immediately after the activity about what they found to be acceptable, which is captured by four different themes below.

Sense of community. This theme captures written statements from Aboriginal students about bringing Aboriginal people together and strengthening a sense of community among them. Aboriginal students expressed that they liked the sense of community that the activity created. Specifically, students thought the activity provided an opportunity to, "Share space and ideas with Aboriginal people" (PO). They also noted that they "liked the community feeling" (DG) of the activity, and liked being “... among [Aboriginal] healers” (RT). As well, students liked hearing the "...Aboriginal speakers" perspectives” (WN). This is in line with the cultural connection theme that was found in the first phase of the study. Specifically, in the first phase of the study, participants spoke about how important their cultural connection is for their own wellbeing, and having a sense of community and/or learning from other Aboriginal people may have been one way Aboriginal students felt connected to their culture during the activity. This is also in line with literature emphasizing the importance of culture in the healing process within Aboriginal contexts (e.g., Dell et al., 2011).

General likes. This theme captures written statements among Aboriginal students about various things that they liked about the activity. Aboriginal students noted components of the activity that they liked more generally, including the refreshments, the honorarium, and the fact that the activity was inviting and welcoming. Baskin (2005) has written about the importance of providing food for Aboriginal participants during research and creating a safe space for these participants, as this allows research to be done in a way that respects Aboriginal perspectives 
(Baskin, 2005). In particular, providing food for participants in an Aboriginal context nourishes the body. Once the body is nourished, people are believed to be more balanced and, therefore, better able to contribute in a meaningful way. The same thing is achieved when participants feel welcomed and comfortable in a research setting (Baskin, 2005).

Some students expressed that the questions asked of them before and after the activity were acceptable and one participant mentioned that no changes were needed to the activity. Such feedback reflects the fact that questions asked before and after the activity were piloted prior to the activity implementation. As well, due to the fact that this activity was designed by Aboriginal students and designed for Aboriginal students through the first phase of this project, it is not surprising that at least one participant expressed that the activity did not need to be changed. It is also possible that this participant did not feel comfortable being candid about what he would have liked to have changed. It should be noted that only one participant provided feedback that no changes were needed, but the rest of the participants did provide feedback regarding ways that the activity could be altered, which will be outlined below.

Recommended changes. Participants were asked immediately after the implemented activity about ways the activity could be changed for the future, which is captured by three themes below. Students recommended changes related to student participation, topics that should be added and discussed, and a ceremonial closing of the activity.

Student participation. This theme captures written statements from Aboriginal students who expressed that increased student participation would have increased the helpfulness of the activity. Participants expressed that they would have liked the activity to be more interactive and engaging. Specifically, students thought that conversations should have been more focused and that students should have been able to talk more and ask more questions. Students also expressed 
that more dialogue should have been created among students. One participant in particular explained that she wanted, "More student participation instead of professionals monopolizing the discussion. Although their input was definitely needed and appreciated, it felt like the students were a low priority" (PO). This participant wanted her voice to be heard during the activity, which is in line with the requests of participants in the first phase of the study (i.e., that the activity should not be lecture style, but rather, more interactive).

Topics to add. This theme captures written statements from Aboriginal students about topics that could have been added to the activity to increase its helpfulness. Students suggested that particular topics be added to the content of the activity. Specifically, it was recommended that there be more discussion about how to navigate the mental health system. This is in line with the common clash between Aboriginal and positivist Western worldviews in the current system as was discussed in the first phase (i.e., Aboriginal people often feel as though their worldviews are not recognized and are invalidated when accessing the mainstream system). As well, this topic was on the agenda of the activity, but due to time constraints during the activity (i.e., there were many things to cover as seen in Appendix U or V: The Wellbeing Activity Agenda), the topic was merely skimmed over. Participants also wanted more information about resources at Ryerson, as this is most local and, in many cases, the most easily accessible for Aboriginal students. Participants wanted information on grief, which was not covered in detail during the activity (see Appendix $\mathrm{U}$ or $\mathrm{V}$ for the Wellbeing Activity Agenda). It is not surprising that this topic was requested, since grief is a common struggle among Aboriginal people (J. Dallaire, personal communication, December 21 ${ }^{\text {st }}, 2013$; Spiwak, Sareen, Elias, Martens, Munro, Bolton, 2012). It is difficult to elaborate on what exactly participants wanted to cover regarding grief, as this was not expanded upon in this participant's feedback form. Participants wanted to talk about 
"other tribal beliefs or values" (PO), which was also not covered during the activity and is fitting with the cultural connection theme that was discussed in the first phase, specifically, how students did not want the cultural component to be compromised and how cultural connection has been found to be closely tied to wellbeing (Chandler \& Lalonde, 1998; Dell et al., 2011). It also taps into the desire from students to acknowledge the cultural diversity of different nations and teachings that various communities have. Students thought that each speaker should have had more specific topics to speak about in order to more smoothly facilitate the activity.

Cultural closing. This theme captures written statements from Aboriginal students, who expressed that having a closing ceremony upon ending the activity would have increased its helpfulness. Participants expressed that they would have liked to see the circle closed using particular traditions, including smudging. One student in particular noted that she wanted "planning for the immediate future - closing the circle by doing this" (PO). This is again in line with the cultural connection theme found in the first phase, as students discussed wanting culture and traditions to be present during this activity and how these traditions should not be compromised. In the first phase, participants discussed how cultural connection enhances their own wellbeing, further emphasizing the importance of including cultural components in the activity and, therefore, provides insight regarding why participants commented on this.

\section{Knowledge to Action}

As discussed above, knowledge to action is a model outlined in mainstream knowledge translation (KT) literature and has two phases: knowledge creation and action (Graham et al., 2006). It is thought to explain how people use knowledge that they receive (Graham et al., 2006). Aboriginal students were asked questions one month following the implementation of the 
activity in order to understand whether they retained knowledge gained from the activity and determine if they were putting any of the knowledge gained from the activity into action.

Knowledge retention. This metatheme captures how much Aboriginal students were able to remember/retain from the activity. Specific areas of retention are discussed in detail below. A month later, participants were asked questions about how much they remembered from the activity. They were asked to rate how much information they remembered on a one ('none at all') to five ('a lot') Likert scale. Participants reported an average rating of 4 (i.e., 'a good amount'). Participants were also asked how much confidence they had in managing their own wellbeing as a result of the activity on a one (none at all) to five (a lot) Likert scale. Participants reported an average rating of 3.5 (i.e., between 'some' and a 'good amount' of confidence). Participants were asked to elaborate on what they had learned from the activity, which was captured by five themes below.

Coping through cultural connection. This theme stemmed from participants' discussions about what they learned from the activity that related to coping with mental health concerns/wellbeing through their culture or cultural connection. Participants stated that they learned coping strategies that were specific to Aboriginal culture. In particular, participants noted that they liked the fact that an Elder was present during the activity and that this Elder provided a traditional perspective on how to cope, such as creating balance for yourself, using Aboriginal spirituality to cope with grief, and using critical thinking skills. Regarding the balance piece, one participant spoke about what she learned from the Elder that she plans apply to her life once she begins working in the health field:

...I would like to get a career in the health field but that's a huge fear of mine that, to deal with the stress of dealing with people's problems. It was intimidating and I felt like it was an obligation and basically [the Elder] said, 'it is'. You have to figure out a way to balance that and to know that I'm not just being nervous about something that I don't 
need to be nervous about. It's good to know that, one: it is a problem and, if you don't deal with it, it is going to be a serious problem and you get burnt out (DG).

This participant is drawing attention to the fact that many Aboriginal people believe in striving toward balance in their lives and that this is even more important when you are working in the helping profession. This participant spoke about learning the ideas of both helping as an obligation and the importance of balance from the Elder who co-facilitated the activity and who validated her fears and concerns, especially related to feeling 'burnt out'. The Elder provided an example from her own life to model the importance of balance within the helping profession, which furthered this participant's learning experience. This quote is also in line with discussions from the first phase of the results (i.e., although achieving complete balance is not possible, we must always be striving for such balance). It is also in line with traditional ways of learning through storytelling and experiential knowledge (Jack et al., 2010), since this participant was able to learn and retain knowledge from listening to the Elder's story about her experiences.

Another participant spoke about his grief and how he learned from the Elder's discussion of grief during the activity, "Yeah, because I knew what she [the Elder] was talking about. Smudging and stuff like that, I find that helps when you're grieving, to do that Native spirituality stuff" (RT). This is an example of a participant maintaining and/or calling upon his cultural connection to cope, as was discussed in the first phase and is in line with Dell et al. (2011) who emphasized culture as healing. It should also be noted that this same participant wrote that he wanted more information on grief during the activity in his feedback provided immediately after the activity and, therefore, it is clear that he needed more from the activity around this.

This same participant explained that, during the activity, he learned from the Elder about how important critical thinking is: 
But I remember the Elder...was talking about critical thinking. She was talking like the teacher from Critical Thinking Philosophy. She was talking about how to have an argument and that you have to back up your arguments. She was saying that you have to think logically about what goes on (RT).

This participant's learning about critical thinking was considered to be cultural connection because it was learned from the Elder who was co-facilitating the activity. It is likely that the Elder was trying to teach participants to advocate for themselves using critical thinking. Aboriginal people are considered to be a marginalized population who are often oppressed by the other social groups and, therefore, advocating for oneself is an important strategy to learn, especially since voices of Aboriginal people are often not taken seriously or listened to within a mainstream context (Poonwassie \& Charter, 2001).

Participants also explained that they learned about Aboriginal specific agencies from the activity that they can go to if they need help and want to heal using their cultural connection: "I remember a lot about what they were saying about traditions and the agencies". The speakers at the activity explained their agency and also spoke about mental health from an Aboriginal perspective, more generally, which is likely what this participant is referencing. One participant remembered a story that a speaker had told and decided to adopt that message into his own life: “He [a speaker] had a different plan. He left his work separate from his family life...Yeah, I learned quite a bit from that guy [the speaker] when he talks about his work and his family. He separates that" (RT). This quote taps into the balance concept, as it is about taking care of yourself, rather than losing yourself to your work or focusing on only one aspect of yourself and your life.

From the results outlined in the first phase of the study, it is not surprising that participants remembered culturally specific coping strategies from the activity, as they had previously expressed, in the first phase, that cultural connection is used to promote wellbeing. 
Although, there were many individual coping strategies expressed in the first phase of the results, there was more discussion of cultural revitalization in the second phase (as will be outlined below). Coping strategies may be moving toward being more culturally specific and communitybased for Aboriginal Peoples (Gone, 2011), which may explain the robust presence of this theme.

Community-based coping. This theme stemmed from participants' discussions about what they learned from the activity that related to coping with mental health concerns/wellbeing through their community/through their connection to others. Participants spoke about ways of coping that may be used in isolation and could be considered mainstream, but that were specifically learned through other participants' storytelling during the activity. Some of the strategies that participants learned and remembered were breathing exercises, having good nutrition, exercising, and how to combat negative thoughts through self-talk. One participant specifically spoke to these strategies:

I learned about the breathing, good nutrition, exercise...Oh, and someone disclosed her thought process and hesitation in say, going outside to make an appointment and then what she did to combat those negative thoughts. I thought that was really interesting. A lot of it was positive self-talk, and that was really beneficial to hear....(PO)

Although the above quote describes strategies that are often used alone and are often taught in the mainstream system, it should be noted that this participant learned strategies from a community member, since she specifically referenced the strategy that was shared by someone during the activity. Learning through storytelling and from other community members is how knowledge was gained in a traditional Aboriginal context, which was the primary means of knowledge transmission from generation to generation (Baskin, 2005; McKeough, et al., 2008). Knowledge transmission through oral methods has decreased among Aboriginal communities over time and, therefore, there is a lack of knowledge in particular areas that are considered an 
important part of Aboriginal culture (e.g., Aboriginal languages; Norris, 2007). Without

knowledge transmission, Indigenous knowledge is at risk of being lost, and without knowledge, Aboriginal culture and identity becomes increasingly difficult to sustain, impacting wellbeing among this population.

Participants noted the value of seeing how other people cope with their problems and the utility of having a discussion during the activity about trying out different strategies if something is not working. One participant provided two quotes to demonstrate this:

I guess I learned about the way people cope with their own unique problems. I think it's valuable to see how people deal with problems and where they're coming from. So, if someone was mad about something, even though I might never experience the problem they had, it was insightful for me to see. Well, if I'm ever mad about something this might be how I may feel and this is some of the stuff that I might have to consider. So, in that way, it was really useful, I think (DG).

This participant highlights the impact of hearing other people's experiences of coping with various struggles, which helped her to remember to have more empathy for others when they are feeling a certain way (e.g., angry). This is in line with traditional ways of learning within an Aboriginal context, as this was traditionally (and is still) done through storytelling (McKeough et al., 2008).

I think that was a really good point you made, if something isn't working for you, then try something else. You don't have to stick with it forever. If it's not working for you, move on. It's really hard when you try a lot of things and nothing works, and then you are just like, 'well what now'? (DG)

This same participant demonstrates her learning about having options regarding coping/healing, and that she can choose between different strategies until she finds something that is helpful to her. She described the difficulties and feelings of helplessness when one realizes that their options are running out, which emphasizes the importance of having many that one can try. It is important that Aboriginal post-secondary students feel as though they have resources, as 
this has been lacking for generations (Harding, 2005) and, therefore, a lack of resources might be a common default assumption for Aboriginal people due to a long-standing pattern of such needs not being met by the system.

Participants learned individual coping strategies, in addition to coping through their cultural connection. However all of the individual, or what could be considered 'mainstream' coping strategies, were learned through a traditional means. A combination of individual and culturally/community-based coping strategies are currently being used within this Aboriginal community, as was seen from the first phase, and aligns with the collective-individual continuum that applies to an Aboriginal context (McShane et al. 2006).

Resource knowledge. This theme stemmed from participants' discussions about what they learned from the KT activity that related to learning what resources can be helpful for a mental health/wellbeing concern. Participants spoke about what they learned from the activity with regard to resources, indicating that they remembered this information even a month later. Participants liked hearing about the different agencies that were presented by the speakers who came to the activity and they liked the resource booklet that was provided to everyone who came to the activity: "I think the resource book you made was really great" (DG). Due to the fact that Aboriginal people often have limited resources (Benoit et al., 2003; Harding, 2005; Jiwa et al., 2008), students noting the importance of a resource handout being made available to them is in line with the literature and students seemed to learn from the discussion on resources during the activity.

Reinforced knowledge. This theme stemmed from participants noting that some of what was covered during the activity reinforced the knowledge that they already had. One participant 
noted that she already had a lot of knowledge about various resources because she had already accessed a few services:

Um, I think that I didn't learn anything because I already used those [resources] when I was trying to get mental health help. So, I kind of already knew that those resources existed. But I think that for someone, like a younger version of myself, had I known then what I know now, it would have made a really big difference... so that I wasn't shooting in the dark or trying to grab whatever was available and accepting what's available. (PO)

This participant is also noting that, although the discussion of resources during the activity wasn't very helpful to her (because it wasn't new information), it would be helpful to 'a younger version of herself' or to people who hadn't sought out or accessed these services like she had. She also demonstrates her resiliency and ability to advocate for herself, since she was able to seek these resources out for herself during a time when she was struggling. This participant noted that it was good to be reminded of other perspectives on wellbeing:

...I would like to say that some things were reinforced. Also, I do have some knowledge, but there are knowledges that I have, which have been related to a minority position. So, hearing other perspectives made those knowledges rise again to a rightful place. I'm talking specifically about the role of language and culture and especially about the history of my people who have really fought for a place in terms of being recognized. I would say more minority cultures, because there are dominant Native cultures and I'm thinking specifically of Cree and Ojibway, and my opinion is that people know more about those cultures and not aspects of my identity, for example. So, other people speaking to those identities, it really reminded me of how important it is for a person to be well, to have their histories known to themselves, and to share and to participate in part of a community. (PO)

The quote above draws attention to the fact that within the Aboriginal population, there exists minority Aboriginal populations, such as Métis and Inuit, as well as particular First Nations identities that are rarely acknowledged. She noted that, in spite of the lack of acknowledgement of these minority Aboriginal identities, it is important to understand who you are and your history, to enhance wellbeing. This participant explains above that the activity 
reminded her of this through the discussion of other, more common Aboriginal identities and knowledges that stem from these identities, which she appreciated.

A participant spoke about being reminded about how important breathing is as a coping strategy, even though she knew about this before the activity:

It's not just a matter of relaxing oneself, but it's also a matter of reconnecting with the acts of inhalation and exhalation and holding that, connecting to that, which connects us to our bodies. So that was an emerging knowledge for me. It is part of my new understanding. I'm also hearing it from other disciplines such as mindfulness meditation. (PO)

This participant notes above that, although she was aware that breathing can be a helpful coping strategy, she learned some helpful nuances of this strategy to make it even more beneficial for her when she uses it. She is also drawing attention to the fact that she has learned this strategy from various knowledge sources, including those that are Aboriginal specific as well as not Aboriginal specific, such as mindfulness meditation. She explains how important the strategy is in connecting her to her body, which might bring together all aspects of her being to facilitate a wholistic sense of self in the moment.

Participants spoke about how the activity reinforced the Native ideology, which was important to them: "It just reinforces that Native ideology. It reinforces what the Elders say, because I listen to the Elders when they talk about spiritual stuff. It's good to hear them" (RT). This quote and the reinforced knowledge theme, more generally, is in line with a point raised by a staff participant in the first phase about how people may need to hear teachings multiple times before they really understand or can apply it to their lives. The theme of reinforced knowledge may directly tap into this idea, since participants might have come to the activity with knowledge about various topics discussed during the activity, but the information provided during the activity could have provided a new or different perspective on what they might have already 
known, as is shown by the breathing example given by the participant above and another participant's statement of gaining a better understanding of Native ideology around mental health. It may also be the case that when information is embedded in Native ideology and culture, the ideas become more solidified and/or participants are more receptive to these ideas.

Having difficulty remembering. This theme stemmed from participants noting that they were having difficulty remembering what was covered during the KT activity or even remembering the KT activity itself. Some participants had difficulty remembering aspects of the activity during the one-month follow-up interview, which was demonstrated either through direct statements of "I can't remember" (RT) or asking questions such as, "Did we close the circle?" (PO), "What were we sharing?" (RT), "Was that when we passed the feather around?" (RT). One participant specifically stated, "I don't remember what that meeting was about" (RT) and "I've kind of forgotten about it because all I've been doing is going to a different class, this class, that class..." (RT). Although most participants were able to remember the activity and give examples of things they had learned, the quotes above demonstrate three possibilities: (1) follow-up interviews could have been conducted earlier than one month after the activity implementation, even though a month has been used in the knowledge translation literature (e.g., Sudsawad, 2007); (2) there may be elements of the activity that could be changed to help participants retain such knowledge for a longer period of time, some of which were suggested by participants and will be outlined below; (3) the activity was not engaging enough for students to be able to differentiate the activity from a lecture-based class.

Components that aided knowledge uptake. This theme stemmed from participants noting particular aspects of the activity that helped them learn and remember information shared during the activity. A month after the activity was implemented, participants were asked about aspects 
of the activity that helped them to internalize and retain the knowledge provided to them during the activity. Participants reported the following portions of the activity to have helped them learn and retain information: Food being provided, resource booklet, well organized, the small number of people in attendance, and those who spoke at the activity. Participants specifically referenced the resource booklet: "Yeah, I think when you have it all in one section and you have lists of how to access it and numbers to call, something like that is really helpful. It was really well organized” (DG), “...because then you have options and it’s not just that someone recommends somewhere and you go there. It's nice to see where you can go. You have a choice to go somewhere", and "I think the package [resources booklet] really made it" (DG). Again, the fact that the Aboriginal population in Canada is an underserved population (Benoit et al., 2003; Harding, 2005; Jiwa et al., 2008) and Aboriginal students in particular may have difficulty accessing resources, it is understandable that the resource booklet provided to Aboriginal students would be seen as so relevant and appreciated.

Another participant elaborated on why she thought the number of people in attendance was helpful in her learning and retention of information a month later:

I think the size...I know you sometimes plan for a larger group, but I thought the smaller group was really good. Like having a small group you can have an opportunity to build a relationship with someone. I think that's more what Native people like. Not, let's throw people in a room and eat things and you know, vent our problems. I mean there's nothing wrong with that, but for the learning part of it, a group of four or five speakers is really good. (DG)

The above participant cites the importance of building relationships as well as its role in learning new information. She noted how relationship building was made possible during the activity because of the small group of people in attendance. Relationship building is the foundation of Aboriginal culture (Baskin, 2005; Battiste, 2000) and, therefore, this quote and the preference for a small group, is very much in line with the Aboriginal way. 
Recommendations to aid in knowledge uptake. This theme stemmed from participants

noting some recommendations regarding ways the activity could have been changed to increase learning and knowledge retention. A month after the implementation of the activity, participants were asked to recommend any changes they thought would have helped them to better internalize knowledge during the activity and retain such knowledge. Participants suggested changes that may help with knowledge application, including structured questions to be added after each speaker presented, the activity should begin later in the day (i.e., at lunch), the activity should be condensed or split into two sessions, information should be written on a flipchart so that people can see and hear the information provided, there should be more of a focus on wellbeing, different speakers should come, and the circle should be ceremonially closed at the end of the activity. One participant suggested adding a peer activity:

I understand that we were pressed for time, like there was a timeline and we only had so much time, but I would have liked, if it was intended to be a peer activity, there could be more discussion around what has worked for us in terms of accessing resources. I think I was kind of confused when I was in that activity, whether it was peer centered, or if it was us listening to speakers. (PO)

The quote above speaks to the desire of participants to have had more of an active versus passive role in the activity and wanting to have their voices heard, rather than merely listening to professionals. It also highlights the desire for storytelling among peers, which is in line with Aboriginal culture (Baskin, 2005). The above quote also taps into the fact that this participant was confused regarding whether the activity was supposed to be peer-centered or if there was a different aim to the activity and this, therefore, should have been made clearer. It was also recommended that the speakers be better advertised, so that people don't come to the activity expecting something different:

It is rather difficult because, in that setting, I felt like the other students, myself included, were hesitant to talk more about ourselves in such a personal way, and it could be 
because a lot of people in that group were in the helping profession and maybe... Okay, I should only speak for myself. I'm going to speak only for myself. That community is so small and even though it's small, I don't know who they [the speakers] are. I felt like I was censoring myself because I'm thinking about, when I do start my career and I meet these people, and having that background... (PO)

The above quote demonstrates the stigma of mental health that is still so highly prevalent among Aboriginal and non-Aboriginal people. The participant above expressed her discomfort in sharing too much in front of people with whom she may be working with in the future, for fear that she would be negatively judged in sharing information about her own wellbeing. This is a common concern within small communities and Aboriginal community members often find themselves in dual relationships with one another. Therefore, this participant's concern is one to be seriously considered if this activity were to be implemented again. She also expressed that she may have learned more if she didn't feel the need to censor herself during the activity. It was also recommended that an assignment be added to the end of the activity, asking people, 'what could you practice?':

Maybe like an assignment or something. Or at the end of the group you could say what are some things we could try practicing? Or what is one thing you could take from this that you will try to do? I think I did that for myself with the breathing. I remember, inhale, 1, 2, 3, 4, 5, 6 and exhale 1, 2, 3, 4, 5, 6, 7, 8. It gives me an exact number, it's not just inhale, exhale. Even counting the numbers I find soothing. It's very structured. Structure in a chaotic world. (PO)

This quote is in line with 'homework' or 'practices' given between sessions during various psychotherapies (e.g., Cognitive Behavioural Therapy) and it has been found among non-Aboriginal populations that 'reminders', which is the purpose that homework could serve, to increases the salience of information provided to be better retained (e.g., Grimshaw, et al., 2002). This quote is also in line with the emphasis of experiential knowledge within an Aboriginal context (i.e., Aboriginal people tend to report learning well through observing and then doing what has been taught; O'Connor, 2010). This participant is also drawing attention to her need for 
structure in a very unstructured and 'chaotic' world, which the breathing technique helps her to achieve. Another participant suggested that information be added to the resource booklet that was handed out to students, specifically, the section of the booklet that outlined questions to ask their general practitioner when prescribed medications for a mental health issue as well as additional resources for the booklet:

I guess I just wanted to add to the booklet. One of the questions when you talk about when you make the decision to try using medications, I thought was really good. I know in the Native community there is a lot of distrust around medication, especially psychotropic drugs and when you had that, I thought it was really good. But I guess one question I would add would be, 'what kind of response can I expect from the drug and how long can it take?' Because some drugs you need to be taking for months before you get a reaction and for someone with immediate anxiety, they might not realize that if it's not going away within a week, then it's not worth taking, you know? Then they can make the decision not to stick with it. So if they knew how long it would take... (DG)

This quote draws attention to the mistrust within the Aboriginal community around using medication as well as the lack of knowledge around this intervention within Aboriginal communities. Therefore, this participant recommends additional considerations and questions to ask that should be added to the booklet, so that Aboriginal students are well-prepared with questions to ask when being prescribed medication, which is often associated with discomfort, confusion, and mistrust. This participant suggested other additions to the resource booklet:

Oh yeah and I wanted to say, your resources were really good, but I don't think you had the school stuff, like the Access Center. I didn't realize that the Access Center could help you so much. It's not just exam testing, they will really help you with whatever you need. They are so kind. (DG)

This participant draws attention to resources at Ryerson University and how important it is to include these resources in the resource booklet, even if they don't specifically target mental health. Addressing academic difficulties will play a role in increasing wellbeing, especially when adopting a wholistic perspective of mental health. 
Knowledge use/action. This meta-theme captures participants' discussions of their use of knowledge gained during the KT activity. Specific ways in which participants reported using this knowledge are captured by the themes below. Participants were asked questions a month after activity implementation about whether they were in fact putting any knowledge gained during the activity into action. They were asked how much the strategies or knowledge gained from the activity helped them to manage their own wellbeing on a one ('none at all') to five ('a lot') Likert scale. Participants reported an average rating of 3.5 (i.e., between 'some' and 'a good amount'). Participants were also asked to elaborate on how exactly they put the strategies or knowledge they learned during the activity into action, which is captured by two themes below.

Using cultural connection to strive toward balance. This theme captures participants' discussions of using their culture or cultural connection for increasing their wellbeing, through either being reminded of this strategy or due to new learning gained from the activity. Participants spoke about using culturally specific coping strategies since the activity. Specifically, participants reported smudging more and incorporating balance into their lives. One participant in particular noted that she has been thinking more about balance in her day-to-day life:

Oh yeah, she [the Elder] was saying that it was something that still bothers her... She [the Elder] seemed really serious about it, that if you don't deal with it it's... Because usually she's [the Elder] not telling you what to do or something. Or she'll [the Elder] talk about something in a vague way so that it's not like this is what you have to do. I'm used to people saying that you deal with problems on your own, in your own way, but this seemed like something that everyone deals with. It's pretty universal. I think seeing that she [the Elder] had to deal with a lot of stress and do things she [the Elder] didn't want to do in order to avoid stressful situations like that, I think that was impactful. (DG)

This participant describes her learning from the Elder regarding the importance of having balance in your life and to deal with your problems in a way that will promote this balance. She explained the story that she heard from the Elder during the activity and how influential this was 
for her. Part of this influence stemmed from the way in which the Elder provided this information (i.e., being very serious and direct about this information as well as providing her own story about how she dealt with her lack of balance in the past). This quote taps into the importance of storytelling and experiential knowledge within an Aboriginal context and how using both methods of communication can increase the salience of the knowledge being provided. She goes on to state that she will use this knowledge in the future, which is not included in this quote above.

Individual coping strategies used. This theme captures participants' reports of coping strategies they have used since the activity that are often done individually (i.e., without the presence of others). Participants reported using coping strategies that are considered to be more individual, rather than culturally specific or community-based. In particular, one participant spoke about using breathing techniques on a daily basis: "A few times a day, especially now because it's midterms and final papers" (PO). This participant references the timing of being reminded of this strategy, since she could use the breathing technique regularly over the course of her exams. This participant noted that she would like to use positive self-talk more consistently: "Well even talking to you, having remembered the positive self-talk, that's something I've gotten out of the habit of, so hearing it again and how well it worked for that woman [activity participant], it would be really helpful if I get back in the practice of doing that" (PO). It should be noted that, although both examples may be considered to be individual coping strategies, they were both learned through storytelling during the activity (i.e., a traditional communication strategy used in Aboriginal contexts).

Another participant explained that he had learned and begun to separate work from family time since the activity: "To separate school from my leisure time or whatever...That's 
how I would cope with stress. Like I do all my school work here and then go home" (RT). This participant also noted that he has tried to be more observant of people since the activity and to try to listen for people's truths: "Well, when I listen to people in charge somewhere I try to see if they're speaking the truth or are they speaking from the heart. A lot of teachers are like that, they speak from what they know but sometimes somebody is just putting up an act" (RT). This quote taps into the trust factor and how this participant plans to try to decide whether or not a person can be trusted by the way they may carry themselves or their demeanor, as this is important to him.

These quotes are in line with the individual-collective continuum. Aboriginal communities are often seen as collective; however, they also have an individualistic component to the culture (McShane et al., 2006). Due to the effects of historical trauma and the dominant positivist Western culture, some Aboriginal people use mainstream coping strategies only. Through cultural revitalization that has continually gained momentum over time, culturally and community-based strategies will likely continue to increase among Aboriginal people.

\section{Global Messages, Current Challenges, and Ideas for the Future}

At the one-month follow-up interview, participants were asked questions about their overarching messages from the entire project, as well as ideas regarding the present and future state of mental health knowledge. Participants spoke about global messages that they received from their participation in the project, anything that they perceived as current challenges in the area of mental health or the mental health system, and ideas regarding the future state of mental health or the mental health system.

Global messages. This theme captured any global messages that participants reported receiving through their participation in the project. They were specifically asked about the 
global, overarching messages they received from the entire project, which are captured by two themes below.

Cultural messages. This theme captured participant's discussions of global, overarching messages that they received from the project that were related to Aboriginal culture or perspectives, specifically. In particular, participants spoke about how mental health is stigmatized in the mainstream system, but how this is different from an Aboriginal worldview: "Got the message that mental health is stigmatized, but that we are coming from a worldview that doesn't see the stigma and that there is a focus on wholistic healing" (WN). Although many participants spoke about mental health stigma as being a significant issue within Aboriginal communities, this participant is drawing attention to the traditional perspectives of mental health within Aboriginal communities prior to colonization, which did not apply stigma to mental health, as mental health was viewed as a natural and normal part of one's identity. Another participant received the message that Aboriginal people need to remember their community and who they are: "I would say, remember where you come from, remember that you are part of a community who is strong and resilient and that we all have inherent strengths" (PO). This participant is positioning Aboriginal people as both strong and resilient, as part of this strength stems from knowing who you are. Both quotes capture discussions that occurred during the Wellbeing Activity and are in line with Aboriginal worldviews.

Individual messages. This theme captures participants' discussions of global, overarching messages that they received from the project that were related to individualistic ideas, such as individual coping strategies. In particular, one participant explained that she learned that you have to deal with your problems:

I guess just that...you have to deal with your problems, pretty much, and there's ways to deal with it and it's not always just one thing. You have options and you can do different 
things and you can try different things. Not everything is going to work. I thought that was really nice that you had different things and it wasn't just one message. I think it was pretty complete on what you can do and what your options are. So it was nice. (DG)

The participant above specifically speaks to the resource booklet, which also outlined different coping strategies that can be used. This participant was very appreciative of having all of this information in one place, so that she could have options regarding what to do and where to go for help, which is in line with the lack of resources that Aboriginal people often have or perceive they have. Another participant noted that it is important for people to speak from their heart: "Just to make sure people are speaking from the heart, I guess. To make sure they are telling the truth and not just putting on a show to impress or something" (RT). This quote taps into the mistrust element among Aboriginal communities and how this can play a role in what information is divulged to others, which may impact resource access among Aboriginal people.

Ongoing challenges in the area of mental health. This meta-theme captures participants' discussions of current challenges surrounding mental health and the mental health system. Participants were asked questions about how they perceived the current state of mental health knowledge among Aboriginal students and many spoke about ongoing challenges in this area. This is captured by three themes below.

Clash of Aboriginal and Western understandings. This theme captures participants' discussions of the ongoing clash between Aboriginal and Western perspectives of mental health and how this is a current challenge in the area of mental health. One participant noted, “...hard to focus on traditions in a Western context. We understand wellbeing from their [Western] perspective, but it's difficult to maintain [the Aboriginal perspective] in the Western system" (WN). This participant draws attention to the dominance of mainstream perspectives and culture and so, being immersed in this context while trying to maintain connection to an Aboriginal 
perspective, proves to be very difficult. Another participant explained, "I think Aboriginals understand their own wellbeing when they are amongst themselves. But when they go out into the white man's world it's different. It's kind of hard to get along with" (RT). These quote demonstrates the difficulty that Aboriginal people experience in having their worldviews and perspectives on mental health validated and recognized while residing in a Western or mainstream system. The quote specifically speaks to the fact that Aboriginal perspectives on mental health are understood among Aboriginal people, but can be fleeting once in the 'white man's world', which drew further attention to the dominance of mainstream perspectives and culture, especially since there is a lack of acknowledgement of Aboriginal people and perspectives within this culture. This clash was also discussed in the first phase of the study.

Mental health stigma. This theme captures participants' discussions of the ongoing stigma that surrounds mental health and how stigma is a current challenge in the area of mental health. Two participants explained the challenge of stigma as related to mental health issues. Participants explained that youth, specifically, feel the stigma; that people are afraid to address mental health issues because they don't feel safe to do so; and that there is movement toward accepting mental health issues among young people, but there is still a lot of stigma around getting help. These points are supported by the quotes below:

I think young people are more accepting of having problems but there is still a lot of stigma around getting help. From my experience some people are almost defensive about saying what they have... There's so much stigma around having learning disabilities or mental illness that they'll own it, they'll say they have it, but the next step of, okay I have it, it's not a big deal and where do I go from here? That part is kind of missing. (DG)

So it's a big difference now from where we used to be and young people are okay with it. They're okay with getting the help and going to the Access Center and being registered. But the coping mechanisms, getting help for themselves, that part is missing. (DG) 
This participant is talking about how far we have come with respect to people admitting that they are struggling or even taking action by accessing help, but that the coping mechanisms, resources available to actually help these individuals, as well as willingness to access help when it is available is still lacking. She positioned youth and their attitudes toward mental health and wellbeing as the catalyst to greater acceptance of those who are struggling with mental health issues. Community members commonly view youth as the future of Aboriginal people (Alberta Education, 2002) and as having the ability to move Aboriginal people forward in a positive way.

Improvements needed in the area of mental health. This theme captures participants' discussions of the ongoing need for change in the area of mental health such as adopting a different perspective on the issue. Participants spoke about what needs to be done in order to address ongoing challenges in the area of mental health. Specifically, one participant explained that there is still a lot of work to be done, especially around intersecting identities, selfacceptance, decolonization, and oppression:

But when I think of members of my family who are teenagers or pre-teens, I think there is still lots of work to be done. Not just around our Aboriginal identities, but also other intersecting identities. For example, two-spirited or LGBT Aboriginal youth are sadly being left out and more work needs to be done around self-acceptance, but also communities decolonizing themselves or oppressing those who identify as two-spirited (PO).

This participant draws attention to a topic that was briefly discussed during the first phase of the project: compounding stereotypes, where those who have a mental illness in addition to being Aboriginal experience greater stigma. This participant drew attention to compounding stigmas of being Aboriginal and identifying as two-spirited. She called for a shift toward selfacceptance and decolonization of communities, as "intersecting identities" was not traditionally and issue pre-colonization of Canada (Wilson, 2007). In fact, those community members who identified as two-spirited (i.e., carrying male and female spirits) were celebrated and seen as 
having a very important role within the community (Wilson 2007). In particular, they were seen as "bridge makers" between the spiritual and the material and between Aboriginal and nonAboriginal (Wilson, 2007).

There was also discussion about community development and how this needs to move forward: "If someone doesn't feel part of their community or part of their family, that has a tremendous and horrible effect on their mental health and wellbeing. So we're behind. On a scale of one to five I would say we are a two" (PO). This quotes speaks to the need to rekindle the sense of family and community, as they may have become fragmented through colonization and historical trauma. Through the reconnection to family and community, wellbeing among Aboriginal students and communities can be increased.

Another participant spoke about the need to communicate coping strategies and resources, so that mental health issues are actually addressed: “...but the next step of, okay I have it [a mental health issue], it's not a big deal and where do I go from here? That part is kind of missing" (DG). This quote taps into the lack of resources or difficulty advocating for oneself to access resources once acknowledging that they are struggling with a mental health concern that needs to change. Yet another participant spoke about the need to practice Aboriginal culture in various agencies: "We need to be practicing our ways in agencies and at the ground level: Front line work where the clients are and promote Aboriginal worldviews there" (WN). These quotes all call for cultural revitalization, especially in the area of mental health and wellbeing.

These suggested areas of improvements should be noted when developing programs for Aboriginal youth or students in order to address identified needs and move Aboriginal communities forward. Specifically, it is important that these needs be addressed, so that 
Aboriginal students can achieve their educational goals without experiencing debilitating mental health barriers.

Future of mental health knowledge. This metatheme captures participants' ideas regarding the future directions of mental health, including what could be done to change current mental health perspectives as well as people's mental health trajectory. Participants were asked questions about where they saw the future of mental health as well as mental health knowledge going, which is captured by three themes below.

Resources targeting youth. This theme includes participants' discussions of the importance in targeting Aboriginal youth with regard to future mental health resources. One participant spoke about seeing things change already. In particular, she explained that the younger generations are more open to change and how this is being translated to older generations. The quote below drew attention to the need for resources to become focused on young people, since they may be in a space of acceptance and can influence other generations:

The younger generations are more open and they want to change. It crosses over between the age groups so that older people now are more understanding, more respectful and they're more willing. So, I think it's really great to focus on young people who are willing to try new things and share that and be an example (DG).

This above quote is in line with the Idle No More movement, where Aboriginal youth have played a large role in speaking up for their rights as well as the rights of their communities (Idle No More, n.d.). It is also in line with Aboriginal perspectives on Aboriginal youth, which are often that youth are the future, who will move Aboriginal communities forward (Alberta Education, 2002). This empowerment may be reflected in youth's willingness to speak up when they need help. This participant notes that such a voice may impact other generations of Aboriginal people.

Cultural revitalization. This theme includes participants' discussions of the importance 
of revitalizing Aboriginal culture to bring back Aboriginal perspectives of mental health and wellbeing in the future. Participants spoke about cultural revitalization as the future of mental health: "We are revitalizing our culture and our culture is coming back and our traditional understandings of mental health are coming back". This quote is in line with the emphasis on culture and Aboriginal perspectives on mental health that was frequently discussed during the first phase of the study.

Another participant spoke about the role of the media in promoting decolonization:

...Also the media has a large role to play too. I think social media has been really awesome at promoting decolonization theory, for example. Even things like Tumblr, and Twitter, and Facebook, people are learning words and phrases even if they haven't been to university. Also, art is always a good tool (PO).

This participant references a few key media outlets that she perceived as contributing to decolonization and noted that art is an important way through which cultural revitalization can be accomplished. Finally, a third participant spoke about falling back on Aboriginal history and how this can promote wellbeing in the future: "I think that's where...Natives have no history to fall back on. They've got to work on that. Like the war of 1812, they should tell the truth about that one or World War I, when they sent all the Natives out to fight" (RT). Cultural revitalization would facilitate cultural connection among Aboriginal people in Canada and, therefore, this is an exciting theme and could increase wellbeing among this population in the future.

Education as a way forward. This theme includes participants' discussions of the importance of educational attainment among Aboriginal people in order to decrease mental health and wellbeing concerns in the future. Participants spoke about education as playing a role in the future of mental health. One participant in particular, spoke about how Aboriginal people are becoming more educated, which helps them advocate for their own wellbeing: 
Sometimes I've been accused of quote, unquote, being a 'bitch' or being obnoxious about it [mental health] and I've learned to really appreciate those qualities. Those aren't my words but I think our people are getting sick and tired of being demeaned and not being heard and we're getting our foot in the door. We're getting our degrees and we're getting fancy, as people in my community would say. We've been around for thousands of years and we're still alive damn it! So I think there's only a forward momentum right now. (PO)

This participant draws attention to the fact that Aboriginal people are being educated and, through such education, they have more of a voice to which people will listen. Through this voice, they are better able to advocate for themselves and to speak up for themselves as well as others, especially when wellbeing is at stake. She is noting the fact that the Aboriginal voice may be demeaned (i.e., 'bitch' or 'obnoxious'), but that she is embracing those terms, rather than letting them hold her back from expressing herself. This is in line with a discussion by another participant who noted that the Elder co-facilitating the activity taught him about critical thinking and advocating for himself during the Wellbeing Activity, which further supports the emphasis on this coping strategy and the importance of this skill among the Aboriginal population in Canada. 


\section{Results: Follow-Up/Debriefing Phase}

This portion of the results was divided into seven different sections, which represents the questions asked during the key informant interviews. These sections include: (1) Project Impressions, (2) Reflections of the Research Process, (3) Project Roles, (4) Community Partner Needs, (5) Take Away Messages from the Project, (6) Present/Future of Mental Health Knowledge among Aboriginal Students, and (7) Project Impacts on Key Informants. Five key informant participants were involved in this phase and their responses to each question asked of them are captured below.

The results from this phase are presented through a description of metathemes as well as themes found to fall under each metatheme. Not all participants spoke to each theme. It should be noted that initials have been placed after each quote to reference each participant. In order to protect participant confidentiality the initials used are not those of each participant's name.

\section{Project Impressions}

Key informant participants (i.e., Aboriginal staff members of Ryerson University who were heavily involved in the project) were asked about how they thought the overall project and the Wellbeing Activity, specifically, went based on their own impressions and from hearing impressions from other key informants or students. The following two themes were found with respect to key informants' overall impression of the project.

Sense of community. This theme captures key informant participants' discussions of the sense of community that the project built for everyone involved, as it brought community members together. Participants spoke about the sense of community that the project created: "I think as your activities went and built on each other, I think that created a sense of community safety net." This participant draws attention to the phenomena of building a "community safety 
net', since such a safety net did not exist immediately, but was developed over time and as each phase was implemented. This is in line with the development of trust and relationships that are so heavily emphasized within Aboriginal communities (Baskin, 2005; Battiste, 2000). This same participant noted that it was helpful for students to have a community around speaking about mental health:

I have a sneaking suspicion that for some people who have really struggled to talk about their own experience, it may have helped them to have language to do that. Without having spoken with them directly about that. Otherwise, I think they appreciated just being able to have a community around that, in some ways (HL).

This participant provides a caveat to her quote above, noting that she did not directly speak with participants about this, yet she drew attention to the safety that was created through one's community and how having a community around a sensitive topic, such as mental health, can create a safe space for people to talk about how they may be struggling and to receive support. This is in line with the importance of community within an Aboriginal context and the safety that is created within one's community (Battiste, 2000). The quote also demonstrates how community was seen as a concept that spans multiple areas of people's lives. This participant positions community as not only the people in our lives with whom we have relationships, but also as a source of support and understanding as related to particular struggles that people go through in their lives. "Community" seems to represent a common and validating language among members, which facilitates such support and understanding. This participant drew attention to the importance of having language to talk about mental health and how having a community around this topic helps that language to develop. This can again be tied back to the importance of community within an Aboriginal context, specifically, the role of one's community in feeling safe to use mental health language and express one's struggle with mental health, which might not otherwise be expressed without the support of a community. 
Another participant specifically spoke about how students respected that one of their peers initiated and was leading the project:

I talked to a few students about this on a casual basis and for most, it was the first time they were ever exposed to this issue of mental health. They appreciated that it was one of their colleagues; one of their own peers was the one doing a study like this. Your being able to speak to them, I think that made a difference that it was coming from one of their own people within the circle and not someone that was already accomplished. I think that sense of respect brought the knowledge level up (SE).

The above quote notes the novelty of the dialogue around mental health as a topic for many Aboriginal students and, therefore, how important it was to have a student community member initiate the project. Specifically, this participant emphasized how having a student community member implement the project made students feel more comfortable in allowing a community to be built around the mental health topic, especially since students could feel on equal ground with this community member (i.e., since the principal investigator; CJD) was a student herself), rather than a professional, such as a psychologist, who would not be seen as equal. This 'equal ground' notion is an interesting one, since traditionally, Aboriginal culture has emphasized equality among community members, rather than hierarchy, as everyone was traditionally seen as playing a key role (Ontario Curriculum, 2006). This quote demonstrated the impact of colonization, as hierarchy is a Western concept (Smylie et al., 2003). The above participant also drew attention to the respect that students had toward the student community member because she was part of their circle, which further highlights the importance of trust and relationships in both building communities as well as conducting community based research within an Aboriginal context (i.e., it promoted respect and better enabled students to learn and retain the knowledge communicated to them). 
The same participant as above explained that, from his perspective, the project became collective and that, even though the project was initiated by the principal investigator (CJD), other people became invested in the project and significantly contributed to its completion:

But don't minimize the importance of you, planting that original thought. Without you starting it, the other people would not have had the opportunity to participate. You had a good array of different folks and that's important. As it went along, it became collective and encouraged you to continue...I think the point is that the seed, the development of this project, came from you. At some point the branches reached out and touched people and it became a collective approach project (SE).

This participant acknowledges the role of the principal investigator (CJD) in initiating the project and drew attention to the project becoming collective among the community. He highlighted the process of the project becoming collective. Specifically, he outlined the importance of providing a variety of students with the opportunity to participate in the project. Such participation allowed students to become involved in the project and contribute to the project in various ways over and above their participation in data collection, which was a key part of establishing a collective project. This quote is very much in line with the aims and goals of community-based research, which is to work collaboratively with the community, so that members are able to contribute to the project (Baum, et al., 2006). The project aimed to use a community-based approach over the course of the project and followed the tenets outlined above. Therefore, it was an accomplishment for the project to be viewed as collaborative as well as engaging for the community and participants. This quote draws attention to the traumatizing nature of research on Aboriginal communities that was implemented, historically. Such research methods were far from collaborative and did not emphasize community needs, community wellbeing, or capacity building (Hart, 2007). This participant notes the importance of the project becoming collective and, in doing so, draws attention to the progress that has been made over 
time in conducting research with Aboriginal communities in a way that is healthy and considerate of these communities.

Growth/learning. This theme captures key informant participants' discussions of the growth and learning that the project contributed to the Aboriginal students involved. Participants spoke about their impressions of the project as contributing to growth and learning either for students, or for the principal investigator (CJD). Specifically, one participant explained how she saw the study touch the principal investigator (CJD) and contribute to the principal investigator's (CJD) own growth and learning:

...but in talking with you, I know it really enhanced you and your understanding as an academic and an Aboriginal researcher and all those other things. And that is not something that we need not include. Your knowledge is...This is your $\mathrm{PhD}$, and yes it is about community development, and yes it's about supporting Aboriginal students, and yes it's about all of that, but don't forget you are still an Aboriginal student. Your PhD thesis is the cherry on top of all of your learning as a student. It's not that you are done learning. It's [the dissertation is] the pinnacle, it's [the dissertation is] the tip. This paper is the big paper of your educational career, not your academic career, and your learning really needs to be acknowledged also. You are learning a lot about yourself, and your own voice, and those owned spaces, and those really need honoring (GR).

The above quote recognizes the principal investigator's (CJD) learning and stated that such knowledge needs to be recognized, especially since the principal investigator (CJD) is a community member herself and, therefore, her learning and growth will impact other community members due to her interconnectedness with others, as is the Aboriginal perspective (Smylie et al., 2003). The current project aimed to support Aboriginal students at Ryerson University and this participant is noting the fact that the project even impacted the Aboriginal student who implemented the project (i.e., the principal investigator; CJD), both academically and personally and the project will continue through future work of the principal investigator (CJD). The participant's point that the learning and knowledge gained 'need honoring' suggests that the principal investigator (CJD) should acknowledge what she has gained from the project. 
One participant spoke about other students who were involved in the project, not as participants, but as research assistants and collaborators and how the project contributed to their growth and learning. In particular, one participant spoke about how grateful a research assistant was for being involved in the project, as she was initially connected to the project through a staff member at RASS:

She [a research assistant of project] was really appreciative of that [opportunity]! She thanked me for that. I saw her three months ago and she really thanked me for that because she said it was a learning experience in doing that [being a research assistant for the project]. I said, 'Well, I knew you were a good fit for this because [principal investigator' (CJD)] was looking for someone.' Working with you she said, she learned a lot and I think that transfers to the students. Even if you just had the core, it resonated with the students, because I think you had people coming from all different directions. I think it did. I think it's really imperative that students look at mental health as not just being the status quo or the typical view of mental health. It affects everybody one way or the other, especially students because there are outside pressures. (SE)

This quote specifically references a research assistant who helped with the project, how much this research assistant learned from her involvement and how she thanked this key informant for connecting her to the principal investigator (CJD). This can be interpreted as further promoting and fostering the relationship between the research assistant and this key informant, which indicates that this project facilitated relationships among those external to the principal investigator (CJD) and the research participants. The learning gained from this research assistant is in line with the tenets of community-based research, which often includes community members at various stages of the project, and aims to build capacity within the community through such inclusion (Israel et al., 2001; Kelly, 2005; Reilly et al., 2008). The quote also drew attention to the fact that the research assistant's learning will impact other community member's learning, which again emphasizes the interconnectedness of Aboriginal communities and how important this learning is in order to understand the normalization of mental health.

This same participant described the project as 'moving' people: 
The most important part of what you just said there was that it moved you, so it will move other people because you truly believe in what you are doing. That's how I look at my life. I have to believe in what I'm doing in order to reach other people. If I just do it for the sake of doing it, it's not going to happen. You put all this time in, not just for the sake of doing it, you really believe in it (SE).

This participant positions the project as teaching people because the principal investigator (CJD) believed in the project's ability to do so, and how important it was for the principal investigator (CJD) to believe in the project in order for it to impact participants. This supports the view of interconnectedness among Aboriginal people. From an Aboriginal worldview, it is believed that all are connected to one another through relationships (Smylie et al., 2003) and, therefore, the research project will not merely 'move' the principal investigator (CJD) in isolation to the participants, rather, through interconnection and relationships, it will impact participants and possibly extend beyond this to impact those who were external to the project. The participant above also spoke about how he had talked with students informally and got the impression that students had learned from the project in various ways:

Just seeing some of the students relate to it and embracing it. When I spoke to them in the common room, and it was casual, it wasn't formal. I talked about mental health and what you were doing. Within the group some didn't participate but some students did and we just talked about it and we were able to generate some comments on mental health and what their opinions were. To me, that was the important part, that they were able to embrace what you were doing and have some form of understanding. But the important thing, as I said earlier is that every one of us has been touched by this issue in one form or another (SE).

The quote above demonstrates the fact that students learned from the project and how the project reached students who did not participate in the study, since the project elicited conversations over and above the formal data collection portions of the project. These discussions that occurred outside of the formal data collection of the study aided all students in forming an opinion and understanding of mental health and wellbeing as being a normal part of one's life experiences. The quote above also drew attention to the interconnections of Aboriginal 
community members through relationships, as even those who did not participate in the project, learned from the project through interacting with students who did participate. He also emphasized the importance of recognizing that all people are impacted by mental health, which is in line with the wholitsic view of mental health found in the first phase, and how mental health is seen as inseparable from the self through an Aboriginal perspective.

Another participant acknowledged the project as teaching people that mental health is a normal experience, as everyone has mental health and people need not suffer in isolation: "Yes. I think there's normalization. That was the biggest thing that I saw, the normalizing. It's simply a brain and it can get out of balance. We take medication and we take it properly then we're fine. It's like anything else" (FC). This quote is in line with traditional understandings of mental health (i.e., that it is a normal part of all of us) and, therefore, it demonstrates the ability of the project to increase learning and understanding of mental health from an Aboriginal perspective, thereby promoting cultural revitalization, which was a common underlying thread within the third study phase, as will be seen below.

Reflections of growth and learning can be tied to the community-based aspect of the project due to the level of engagement that the project allowed community members. Such reflections can also be tied to the knowledge translation literature including 'sharing what is known about living a good life', which can involve many processes including involving community members to shape research and sitting around a kitchen table sharing knowledge (Kaplan-Myrth \& Smylie, 2006). These two processes, in particular, were implemented during the project, including informal sharing that occurred over and above the data collection portion of the project (i.e., 'When I spoke to them in the common room, and it was casual, it wasn't formal'). As well, the activity was in line with recommendations by MacCaulay et al. (1997), 
which included translating locally based and locally relevant knowledge to the community and having community members translate messages to the community. Recommendations by MacCaulay et al. (1997) are in line with KT guidelines by Hanson and Smylie (2006). Due to the fact that the activity followed recommendations by both MacCaulay et al. (1997) and Hanson and Smylie (2006), it is not surprising that a theme found during the key informant interviews would relate to participant learning and growth.

Common threads between both themes under project impressions were interconnectedness and relationships. In particular, community and safety could not have been developed without trusting relationships with community members. Growth and learning among Aboriginal students were described as occurring, as well as being strengthened, through relationships students had with one another and with the principal investigator (CJD).

\section{Reflections of the Research Process}

Key informant participants were asked about their reflections of the research process, specifically regarding relationship building and the activity evaluation process.

Pros and cons of the evaluation process. This theme captures key informant participants' discussions of both pros and cons of the evaluation research process (i.e., what worked and what did not work). Participants explained that they saw both pros and cons of conducting the evaluation in the way it was done. Specifically, one key informant thought that participants may have had a hesitancy to respond honestly when having the same person who implemented the activity, evaluate the activity; however, if someone else evaluated the activity, they may have also felt uncomfortable, as is explained by the following quote:

As a member of the community, you have to be in the community and it's a give and take relationship. So, there may be a hesitancy to reply...I don't know. They may have had hesitancy, if they felt uncomfortable. I don't know that anyone did. I do know that in our community there has been hesitancy to stand up where people just let things go that 
didn't feel okay for them. At the same time, if it was someone different, who they didn't know [conducting the evaluation], that might actually be worse in some ways. So it's a balance. How to address that concern... I don't have any suggestions. I don't know that having someone else necessarily outweighs the balance in that, versus your other concerns (HL).

This participant begins by emphasizing the importance of being a community member and that you have to participate in the community to be considered a member, as this will maintain relationships (i.e., 'give and take'). She may have been providing feedback to the principal investigator (CJD; i.e., that the she needed to be more involved in the community in order to decrease discomfort among Aboriginal students in providing candid feedback) or noting reasons why it may not work for someone with no relation to the participants to conduct the evaluation (i.e., they would not be a participating member in the community). She also draws attention to the lack of advocacy among Aboriginal students, which is in line with a teaching that was provided by the Aboriginal Elder who co-facilitated the Wellbeing Activity, as she taught Aboriginal students about critical thinking and advocating for themselves. This participant noted that having someone else conduct the evaluation may create discomfort, as this individual would be devoid of relationships with Aboriginal students, which is in line with the emphasis on relationships within Aboriginal research methodology (Baskin, 2005).

Another participant spoke about possible pros and cons of conducting an evaluation on the activity that was implemented by the same person:

I think when you ask the questions you run the risk of two things. Either people who like you being really positive rather than accurate, or people who either didn't like you or didn't like the program being very negative about it. So it is a running risk. I think with someone else asking, it removes some performance anxiety or people-pleasing anxiety. But I think either way it can give accurate information, it may just be that how it is putforth or how it is said may be different. Also, we are dealing with university students who are more apt to be honest and open if asked. As I say that, the other thing that pops into my head is that they might not be [honest] because they may feel a little bit vulnerable or exposed in talking about stuff and then reviewing with the same person again. Lots of times I found that when I was in counselling and people exposed all their stuff they 
usually missed the next session or were a little bit late for the next session because they were feeling...because they talked about things they don't talk about. So they felt exposed and vulnerable in wondering if there would be any difference in my reaction to them...I also think that one of the things that RASS already does as part of its existence is tend to the mental health of students. So, I think it is a much safer place for Aboriginal students to talk about mental health issues because there would be no judgment. It is part of what we provide, right? (FC)

This participant lists some pros and cons of having the same researcher, who implemented the activity, evaluate the activity and expressed some interesting advantages of bringing someone else in to conduct the evaluation, as it might decrease anxiety among students. She also drew attention to the ability of Aboriginal students to advocate for themselves, which is in contrast to the previous quote, stating that community member often refrain from expressing their discomfort when something does not feel right. The participant above seems to have expectations of Aboriginal students in that they are able to express themselves openly and honestly. This participant noted an important potential downfall of the same researcher implementing and evaluating the activity, which she drew from her counselling experiences: making participants who felt exposed and vulnerable during the activity face the same researcher again. The activity and evaluation aimed to be helpful and empowering for students; however, the process of collecting follow-up data may have been inadvertently traumatizing for some students, which is a very important consideration, especially when conducting research within Aboriginal contexts. Although Aboriginal people are incredibly resilient, they come from traumatizing histories and, therefore, the ideas noted by the above participant should have been considered prior to follow-up data collection and should be considered in the future. Finally, this participant drew attention to the importance of community within an Aboriginal context, noting that RASS is a 'safe place' for Aboriginal students to talk about mental health and wellbeing, as a community has already been developed around this topic. 
Relationship building as a key part of the research process. This theme captures key informant participants' discussions of relationships as an important part of the research process. Most participants explained that they believed that relationship building was an important part of the research process. In particular, participants spoke about how such relationship building helped to recruit participants, which is demonstrated by the following quotes:

It was harder for us to recruit for the first activity because people really didn't know what you were doing or who you were. As they started to get to know you, it became easier for us to get them to be like, 'Oh, okay, I'll go!' So they seemed happy to participate and they seemed happy with the experience. I talked to a few of them afterwards and said, 'How was it, was it worth your time?' and they were like, 'yeah, it was good' (HL).

And in the beginning that's why it was hard, right? It was hard because people didn't really know you and they didn't know what you were doing. So it became easier once they got to know you. You were around the space more, they knew who you were, and then people would be like, 'what's this happening?' and I would just let them know what I knew...I mean, in some ways it was kind of...In some ways you could get really theoretical about research methodology, and the community, and stuff like that, but it's [relationships are] kind of what it's all based around. It's [relationships are] part of the circle and you can't separate the research from the circle (HL).

This participant draws attention to the relationship component of Aboriginal culture and how Aboriginal people tend to participate in research projects or other activities when they know the person facilitating such activities, personally, and/or have heard from others that it is worth their time (Baskin, 2005). This is in line with relationships as being a foundational component of Aboriginal culture and, therefore, it is not surprising that students were reluctant to participate in the project before they knew the principal investigator (CJD) or what the project was about. This drastically changed once such relationships were built. Relationships cannot be separated from the research methodology (Baskin, 2005). This is in line with Hanson and Smylie (2006) who suggest that knowledge can only be effectively translated when relationships have been built on respect and trust. 
Other participants spoke about the relationship component and how important it was to honour such relationships throughout the entire project. As well, participants explained that having the same researcher implement and evaluate the activity was in fact an acceptable way to collect such data due to the relationships that had been initially built between participants and the principal investigator (CJD) during the project. These ideas are demonstrated by the following quotes:

When you and I first talked about when you delivered the activity, you told me you went around the circle. You didn't tell me who was there, but when you went around the circle and the students were saying why they were there, they were saying that they were there because you asked them to be there. They were there to support you. If you had sent someone else in to ask their feedback...I can see the idea from a more Western understanding that they may give you more candid feedback, however, they don't have a relationship with that person. Aboriginal research is about relationships, it's about knowing who you are working with and knowing why you are there because of the relationship you've built with the primary researcher. Let's be honest, that is what Aboriginal research should be. The fact that I'm here is because of my relationship with you. I believe in you. So if you had sent someone and said I had to talk to them, I would have talked to them and given them as much feedback, but it would have been different. I might not be as open. I think that the depth and importance of relationships to Aboriginal research cannot be minimized (GR).

This participant is drawing attention to the importance of relationships in Aboriginal research. She is specifically noting the importance of research participants knowing who the researcher is and knowing what the research is about. Similarly to quotes from (HL), this participant taps into the difficulty in recruiting Aboriginal participants if there is no relationship between them and the researcher. This participant further elaborated on the importance of relationships below:

If you had said to me, I'm going to have someone evaluate my work and I would like you to sit and talk with them, I would have. I would have been like, 'of course, for you I'll do it'. But it wouldn't be as honest. I would feel, if I had any critique, I wouldn't give it to someone else. I would want to give it to you and to your face and I would want to explain to you why I felt that. The glowing stuff I could tell them, but if there was something I felt was wrong, I wouldn't be as candid. I would feel shame to tell someone else when I 
don't have the courage to tell you. You are the most important person for me to tell. Does that make sense? (GR)

The two quotes above explicitly explain why it is important to keep a consistent researcher throughout the project (i.e., the existing and established relationships are key). She noted that 'Aboriginal research is all about relationships', which is in line with work by Baskin (2005) and Hanson and Smylie (2006) who both emphasize the importance of relationships in Aboriginal culture and research. She also tapped into the importance of respect by explaining how important it is to provide feedback to people, rather than to someone else about that person. Through respect grows relationships, which is a cornerstone of Aboriginal culture and, therefore, providing feedback directly to the person is perceived by this participant as facilitating a relationship through respect. Such respect is tied to relationships and respect is built through the development of relationships. The quotes above drew attention to the differences between positivist Western and Aboriginal perspectives and how, within Aboriginal contexts, relationships are foundational, but that this is not the case within a positivist Western context. In fact, within a positivist Western context, it is important to refrain from having relationships with research participants and, in many instances, such relationships are considered unethical or, more specifically, a conflict of interest (Sinclair \& Pettifor, 2001).

Another participant drew attention to the clash in Western and Aboriginal worldviews when it comes to relationships in the research process:

So this is where I see a real departure between the Western model and Aboriginal ways of working... You followed your instinct. Why would you give it over to someone else? You are the person who has created the relationship with them and to do that without saying or giving them the rationale that you just provided to me, most people would say, well why would I need to talk to someone else if I did the work with you? Again, if that came up in your defense, it is one of the strengths of the way in which we work, is always to be selfaware and to do that self-reflection. If you weren't doing it, it wouldn't be as strong as I know it is because essentially we don't begin work and then give it over to someone else. If their evaluation is that, it becomes flawed because you are reflecting on your own work 
is again a place of departure because only when you are able to reflect on who you are and the work you are doing and figuring out what you've learned and what you've helped other people learn, then we can't move (MK).

The above quote further explains the importance of relationship building within the research process and how this departs from positivist Western ways of thinking and doing. Relationship building as a way of thinking and doing within an Aboriginal context is viewed as a strength by this participant, representing the resiliency of Aboriginal culture. In spite of the many adversities and historical trauma that have been faced by Aboriginal Peoples in Canada, they have held onto one of the most foundational components of their culture and worldviews: interconnectedness and relationships between all things. The quote draws attention to how important self-reflection is, which is another reason why it was key for the same researcher to implement and evaluate her work. Constant self-reflection is an expectation within Aboriginal culture and is highly emphasized (E. Sackaney, personal communication, September $7^{\text {th }}, 2012$ ) and has been examined by Gone (2009) who studied healing among Aboriginal people from the impact of residential school and found that self-awareness (i.e., looking into oneself) was necessary for desired long-term outcomes (e.g., continual self-growth). The participant notes above that, without self-reflection, 'we can't move' or Aboriginal communities cannot continue to progress and move forward toward self-determination, which is a common goal within Aboriginal communities (Asch, 2002).

In line with the importance of relationships in an Aboriginal context, participants spoke about trust, which is a significant part of having the same person implement and evaluate the activity and is an important component of relationship building:

Well, the most important thing I think you accomplished was the trust factor. Gaining that trust is the key to having a person open up. So, for you to follow-up is showing sincerity and the fact that people saw you again showed that this young lady really believes in what she is doing. The fact is, they would be more receptive to you, I think. 
Just like me, I'm more receptive to you because I know you and I see the effort that you've put in. Those students were probably thinking the same way because they've seen you before. I don't think it's a detriment (HL).

The quote above notes the importance of trust, which provides insight regarding why relationship building is so very key within Aboriginal contexts. In developing relationships, you develop trust and this will increase one's comfort in providing candid feedback. Aboriginal people have historically placed their trust in individuals, institutions and systems that betrayed this trust and, therefore, it is logical that developing these relationships will require time and effort before such trust is established and relationships can be built. The principal investigator (CJD) put time and effort into the relationships that were built over the course of the project for her own personal development as well as for the sake of the project, which was acknowledged by the participant's quote above. This participant also spoke to the message that was delivered to the students with whom the principal investigator (CJD) followed up, which relates to the understanding that the principal investigator (CJD) believes in the project, further establishing trust in the relationships that had already begun. These relationships allowed for the collection of rich data that provided insight regarding how to define mental health from an Aboriginal perspective and what students wanted in a KT strategy to communicate mental health knowledge and resources.

Process recommendations. This theme captures key informant participants' recommendations regarding how the research process could have changed to better facilitate its completion. One participant made recommendations on how to change the research process to make it smoother and more effective, including facilitating communication, having a support person collect or be present for the follow-up data collection, and to create a relaxed space for students to participate in the follow-up interview. The following quote demonstrates one key 
informant's need for more communication and a smoother transition when replacing another key informant's role during the project:

I think in the beginning, because of the transition of [key informant] not being here, I think a lot of it was difficult for me to pick up because I felt a little bit behind. There was an intention of having more conversations after we had that first conversation with [key informant] and I, and then [key informant] was just gone. I was like, 'wait!' So I felt like I missed a couple of pieces in there. But I mean that was fine. I wasn't really sure about what your agreements were with [key informant] and what [key informant] had promised. And that was mostly because [key informant] and I hadn't communicated about that. She told you I would just pick it up and she probably told you that she would talk to me about it and then something unexpected happened. But that happened around a number of things at work that I picked up for her (HL).

When conducting community-based research, there is often turnover with respect to staff positions, as people move on to other positions and new employees come on board. The participant above is noting some difficulties around this. She noted that she and the other key informant did not communicate with respect to responsibilities within the present study, but what is also apparent is that she and the principal investigator (CJD) did not communicate around this either, which could have been addressed. Therefore, it is important to consider ways to create a smooth transition for people who are becoming involved in the research project, as is noted in the quote above. This participant suggests an alternative process in the quote below:

I think if a student came along next year and I was in this place having the conversations that [another key informant] had with you in the beginning, I would feel more in control and able to support you more, recruit more, and be able to offer more of our resources. But it was what it was at the time (HL).

In this quote, the participant expresses her wish to have supported the principal investigator (CJD) more throughout the project, but that she was limited by various factors. Her supportive role could have been supplemented through various means, including more regular communication initiated by the principal investigator (CJD) and this should be noted if this project is ever to be implemented again in the future. 
This same participant made some suggestions regarding ways to ensure that participants were comfortable providing accurate feedback during the follow-up interview:

I think about some of the classes that get offered in community settings. Often they will have the instructor and a support person....At the same time in the classroom. So, if something emotional comes up that needs to be addressed...So, maybe have someone that people don't feel they have to act a certain way around, like the comrade person in the room. I don't know. That's my hypothesis. If there was a big research project having someone in that role may be beneficial (HL).

This quote suggests having a support person present during the interview in order to manage any discomfort that the participant may feel and to promote feelings of safety among participants and further taps into the idea and importance of trust in Aboriginal contexts. Including a support person can be a source of trust for an Aboriginal participant. This participant discusses the potential benefit of creating an informal research setting for participants:

I don't think they would be uncomfortable. I think you would have to set up a very social setting. You could theoretically do it over dinner or a party or something. This is one of the things where community and research has a really difficult time meshing... I find more than anything the casual approach is better. But I don't think they would be uncomfortable if that was your question. I don't think anyone would be uncomfortable if you sought them out. I just don't know how much you would get from it (HL).

This quote suggests creating an informal and social environment for students, as this may help them to feel more comfortable providing feedback about the activity. The suggestions above are in line with Baskin (2005) who recommends culturally safe research methods for use in Aboriginal communities and such recommendations are very different from conducting research in a positivist Western context, as research is conducted in a way that is devoid of relationships. The two quotes above once again tap into the importance of relationships and how the presence of such relationships (e.g., through having a support person attend the interview or creating an informal social environment for feedback) can facilitate the research process in very significant ways (i.e., facilitating candid feedback). 


\section{Project Roles}

Key informants were asked how they viewed their role(s)/responsibilities throughout the project. The following two themes were found with respect to key informant participants' perspective on their own roles throughout the project.

Supporting relationships. This theme captures key informant participants' discussions of their role/responsibilities throughout the project as providing support in various ways. Participants specifically spoke about their roles as providing support around relationship development throughout the project. In particular, one participant spoke about liaising between the principal investigator (CJD) and the community members: "Yeah, I mean my impression was, I was mostly just liaising and to talk it out and talk about you as a member of our community" (HL). This participant is speaking about promoting relationships between the principal investigator (CJD) and Aboriginal students, which, as has been previously discussed, is a key part of the process of conducting research with Aboriginal communities.

Another participant spoke about providing her support by believing in the project and what it stands for: "Well, it [the project] makes sense and it's important and the work that you are doing as a helper is to nurture, and give voice, and give resources, and give knowledge about the impact of colonization. That is what you are doing" (GR). Through believing in the project, this participant promoted the project and also played a significant role in helping the principal investigator (CJD) develop relationships within the community, as is necessary within Aboriginal contexts.

Another participant spoke about his role as supporting the development of relationships between the principal investigator (CJD) and the community, and how important this is in Aboriginal contexts: "I'm just one of those connectors. I was one of those people who was able 
to be suggesting, sharing opinions and be encouraging, and just be able to stress the importance of what you are doing and the outcomes that could potentially happen" (SE). As previously discussed above, relationship building was an important part of the research process within Aboriginal contexts. This participant spoke about his role in facilitating the development of such relationships between the researcher and the community members. All three key informants helped during recruitment portions of the project and supported the project through believing in it and facilitating relationship building between the principal investigator (CJD) and the participants. Their roles as well as their perception of their roles, are very much in line with the tenets of community-based research as it aims to engage community members in the research process (Cornwall \& Jewkes, 1995).

Spiritual/traditional support. This theme captured key informant participants' discussions of their role/responsibilities throughout the project as providing spiritual support or support stemming from traditional Aboriginal ways. Participants spoke about providing support that was spiritual or traditional, such as praying for the project during traditional ceremonies and using traditional knowledge to support the project. One participant spoke about his role in supporting the project by praying for it during a traditional ceremony: "Every time I did a pipe ceremony I thought about you and thought, 'well I hope she is carrying on because this is important"' (SE). This is interpreted as spiritual support because this key informant called upon the spirit world to help move the project forward. A pipe ceremony is a sacred ceremony that aims to connect the spiritual and physical worlds (Wood, 2000). Spiritual support is considered to be a significant source of support within Aboriginal contexts.

Two other participants explained their roles as contributing traditional knowledge to the project as a way to support it: 
I would hope that I was able to say clearly that regardless of who are in those front line positions, our role is to accompany, to stand with, never to lead or to be pushing from behind. We walk with people. An important teaching role is to ensure that our students understand and appreciate being their own self-advocates. I think when we are in those spaces and we can do that, it's only then that you can turn to the person beside you and say, 'Okay, now I need your help'. Essentially, we always begin with, you know yourself, you are the expert on yourself, you know your strengths and your abilities, you know when you're getting challenged and you know best when you are not being heard (MK).

This participant explains that she contributed traditional knowledge regarding mental health to the project. Specifically, she noted that individuals with mental health issues are not expected to struggle in isolation, from an Aboriginal perspective. The contribution of traditional knowledge to the project is an important one, as many Aboriginal students expressed their desire for such knowledge during the first phase of the study. This participant expressed her hope that she contributed knowledge on the importance of self-advocacy, so that students can speak up for what they need from themselves and others. This is in line with the self-advocacy teaching that occurred during the Wellbeing Activity.

This same participant explained where her knowledge had come from (i.e., from her own experiences and from her Aboriginal worldview), which enabled her to contribute traditional knowledge to the project:

... I was able to speak from the experiences of doing front line work with students and that I also spoke from the place of a person who sought out processes and healing opportunities and used both Western and Aboriginal Indigenous models of engagement. For me, I think the benefit came from the Aboriginal Women's Healing Circle that I belong to. It helped me pull things together. I had done individual counselling, I had gone to a group that focused on anger and release, but there were shortfalls of both. Therapy and counselling fell short because the model was so rigid. There was very little place of recognition or acknowledgement of being an Aboriginal person and bringing that intergenerational piece that I'm sure I talked about [during the project], the things we inherit from the people we come from, and the intergenerational trauma and the impact (MK).

In the quote above, this participant explains that, from her experiences, healing models are often very rigid (i.e., rarely emphasizing the Aboriginal perspective) and how she provided 
knowledge on the impact of intergenerational trauma. This knowledge is in line with the current literature, which speaks to the importance of intergenerational trauma in mental health and wellbeing, as well as blood memory. Mental health issues are believed to be passed on intergenerationally and through blood memory within an Aboriginal context (J. Dallaire, personal communication, October $7^{\text {th }}, 2013$; Morgan $\&$ Freeman, 2009).

In both quotes above, the participant reflects on the knowledge she shared during the first phase of the project, which helped to shape the KT activity, as was her role within the current study. The knowledge she shared during this phase supported the project by helping to ensure that traditional knowledge about mental health would be shared during the KT activity.

Another participant spoke about the traditional knowledge that she contributed to the project and how she considered this to be her role. She also saw her role as an Elder and a participant in the project, which was the case:

Well, I saw my role as contributing from an Aboriginal perspective, mentoring and supporting you. I saw it as a continuation of my role at Ryerson, in that if anything goes wrong I would go in there and make sure everything is okay, also as being a participant in it. It felt very rewarding for me (FC).

This participant was a significant source of knowledge and support during the Knowledge Translation Phase of the project and contributed traditional knowledge and experiences to the project as a whole. The role she is describing above is the role of an Elder within an Aboriginal community (Dumont-Smith, 2002) and she is recognized by RASS, as well as many other Aboriginal communities, as an Elder. Ensuring that traditional knowledge was acknowledged and shared throughout this project fosters cultural revitalization, which was expressed by many Aboriginal students as a need during the first phase of the study and is in line with literature that emphasizes culture as healing (Dell et al., 2011). Therefore, both participants who expressed their roles within the project as providing traditional knowledge contributed significantly to the 
project and addressed exactly what Aboriginal students had requested during the first phase of the project.

\section{Community Partner Needs}

This metatheme captures discussions of community needs that this project addressed, began to address, or did not address. Key informant participants were asked whether and how this project addressed any needs within the community. The following three themes were found with respect to key informants' opinions on how the project addressed or did not address community needs.

Addressed capacity building needs through dialogue. This theme captures key informant participants' discussions of capacity building needs that were addressed through the creation of dialogue through this project. All five participants spoke about the project as addressing capacity building needs through community cohesion and dialogue. One participant, in particular explained that the project allowed community members to interact positively with research and that it brought the community together:

Without knowing the content of what you presented [during the wellbeing activity], I think there's an element of, I keep coming back to the social community need in our project, for people to interact positively with research, and each other, and topics that are hard for them, and there isn't a lot of space for them to talk about it. So I think in a lot of ways, looking back on the course of the year, and I'm thinking of specific people I know who participated along the way and how it's good for those people... to have a space. So I think it's good, and it's good to grow our community in that way, and create a dialogue among people, and to get people who may not socialize in classes or in the student lounge together in a room $(\mathrm{HL})$.

This participant notes capacity building among the community around mental health and how the project created a space for such capacity building, allowing community members to learn from one another and from the project regarding mental health and research. She noted specifically, that the project allowed '...people to interact positively with research...', which is 
key, since research has been historically traumatizing for Aboriginal Peoples, since it was done without regard for community needs and without community collaboration (Battiste, 2000; Hecker, 1997).

Another participant echoed the need for dialogue around mental health, as was mentioned above, since such dialogue contributes to building capacity: "I'm glad you did this for the students. It's important that you did it for you, but just to bring the conversation of mental health into that space is really important." This participant emphasizes the importance of mental health dialogue, which is likely due to the stigma that often surrounds mental health. Because of this stigma, Aboriginal students often suffer with mental health issues in isolation and so this participant is noting the importance of bringing in a conversation about mental health to the community to build capacity around these issues.

Yet another participant agreed with the importance of dialogue:

That's why I appreciated your project. We tend not to pay attention to that. So, when you came along I thought, that's great, because people don't fully realize that these students go through a lot. Some of them may minimize it, but as a helper or carer, I've never minimized the stresses of a student (SE).

This participant expresses his appreciation of the project in drawing attention to the struggles of Aboriginal students, which is a key part of building capacity within this community to address needs around this issue. Another participant noted how the research process was focused on hearing from the community and community members' voices through flexibility in collecting people's responses, allowing for rich discussions of mental health of which would be reflected back to the community, aiding in capacity building:

Again, when I think about the focus group I participated in, you came saying this is what I would like us to focus on. For me the strength was that you came into the project understanding that if you really are going to accompany the community and really hear from them, as we did with our focus group, it was about the questions you came with. But then we started to talk about things outside that as well as how and which they intersect. I 
believe that is a strength of using Aboriginal ways of knowing and knowledge, to be flexible and to understand that you may take a bit of a journey but you will come back to what you were asking. But now it's been enriched and you've been given other thoughts and experiences about why they reflect back and need to be included (MK).

This participant explains that the flexibility of the data collection that occurred throughout the project was important because it allowed for the principal investigator (CJD) to hear the community's dialogue around mental health in order to reflect this information back, even if what community members were saying were not necessarily answering the questions asked. Being flexible during the research process allows participants to feel validated and to feel as though their voices are being heard. This is in line with Baskin (2005) who states that research methods need to occur organically (i.e., you cannot force the discussions, rather you need to let them happen) within an Aboriginal context and Aboriginal participants often want to have their stories heard and the research method should be designed to allow this. The quote above is also in line with recommended KT strategies that are specific to Aboriginal contexts, which recommends that knowledge be created by community members and given back to community members by community members (Hanson \& Smylie, 2006). It is through this flexibility that rich data can be collected and that capacity can be further built around coping with mental health issues.

A final participant spoke about the project drawing attention to the gaps in the University around Aboriginal perspectives (as related to mental health, specifically) and that the project provided community members with a space to talk about mental health as a topic: "Oh yeah, I think it did address the needs of RASS. I think just the whole project itself shone a light on the needs at the University. I think that the project in itself created an environment that people could share and talk about stuff' (FC). In learning about the needs of the University as well as creating a space for dialogue, capacity is furthered through having knowledge around which needs should 
be addressed within the University and increasing capacity around coping with mental health issues.

Addressed need to normalize mental health issues and decrease stigma. This theme captures key informant participants' discussions of the community's need to normalize mental health, which had begun to be addressed through this project. Participants specifically noted that the project helped to reduce mental health stigma and began to normalize mental health as is demonstrated by the following quotes: "Yeah, I think what it did was you gave a lot more information. You really helped remove the stigma around it" (FC) and "I think what the project did was normalize. Once again, it's in everybody's family. Was there anyone there [at the activity] that didn't own up to, including the participants as the professionals, if you will - own up to having had mental health issues? Either had, or currently living with" (FC). These quotes are in line with traditional ways of perceiving mental health (i.e., that it is part of everyone) and, therefore, the project addressed the need to normalize mental health and attempted to remove the stigma from it, moving the understanding of mental health toward a more traditional Aboriginal perspective. These quotes are also in line with the first phase of the project, as many participants spoke about the need to remove stigma from mental health and how unhealthy mental health stigma is for one's wellbeing. Aboriginal people often emphasize the need to revitalize and maintain their knowledge systems and, therefore, promoting an understanding of mental health from an Aboriginal worldview was an identified need within the community.

Addressed need for resources. This theme captures key informant participants' discussions of the community's need for resources that had begun to be addressed through this project. Participants mentioned that the project addressed Aboriginal students' need for resources 
by either providing a guide for helping professionals as well as non-Aboriginal people, or by being a resource in an of itself:

...the reason I asked whether this would be published at some point is that this could be a good guide for social workers and caregivers coming up because they will realize the massive challenges out there, but they can still be overcome. Even one little issue, if talked about, a resolution can come about. They know this is the issue, here's what I can do to take care of it and they take care of it and they go on (SE).

This participant explains that the project could teach helping professionals about how to communicate mental health information to Aboriginal students, which taps into the need for external resources to understand and acknowledge Aboriginal worldviews. This is in line with discussions during the first phase of the project, as students spoke about the lack of understanding among health and mental health professionals of Aboriginal worldviews and perspectives, which often leads to Aboriginal people feeling invalidated by the system they might access. This participant is suggesting ways in which the project could impact those in various helping professions and could contribute vital resources to Aboriginal post-secondary students through guiding healthcare professionals.

Similarly to the quote above, this same participant explained that this project could help non-Aboriginal people gain a better understanding of Aboriginal peoples' perspectives:

I think it [the project] will be helpful to the settlers. They need to expand their conceptual approach when working with us. They have to put aside their...they have to take those blinders off. That's another thing, when I tell people what you are doing, I tell them you're creating Aboriginal experts on this issue. Hopefully I'm still there to keep promoting that. The more I get from you young folks, the better it is for me to carry on what I'm doing. I've worked with young people all my life, when I think about it, I started when I was quite young and when I was hurting, but I'm so honored to be where I am now (SE).

This quote explains that the project could be an educational tool for non-Aboriginal people (i.e., 'settlers') in expanding/furthering their perspectives on Aboriginal people and Aboriginal issues. Specifically, he requests that settlers "take their blinders off", which is a call 
for non-Aboriginal people to educate themselves and have an understanding of Aboriginal people. In other words, he requests a move away from ignorance to "see" Aboriginal people for who they are and what their needs are. This aligns with discussions in phase one of the project, when students emphasized the clash of Aboriginal and Western ways of knowing, as nonAboriginal people often do not have an understanding of Aboriginal people's history and culture. He also notes that the project can aid in creating Aboriginal experts, which is another resource need within the community, as those who are knowledgeable in this area are more likely to be able to effectively help those who are in need of mental health resources. He notes that the project has aided him in becoming a better helper and, therefore, a better resource to students, as he has learned from the students, himself. This participant's learning will impact Aboriginal students by providing them with an even more effective resource, which is a definite need within the community.

Another participant noted that she thought the project addressed resource needs within the community because the activity was a resource for students in and of itself: "You offered potential places of support where people could go for free support around mental health issues." It is not surprising that resources were identified as an addressed need through this project because Aboriginal Peoples in Canada are considered to be an underserved population, where resources are often non-existent, inaccessible, and/or culturally unsafe (Benoit et al., 2003; Gray, Saggers, Sputore, \& Bourbon, 2000; Harding, 2005; Jiwa et al., 2008; Shannon et al., 2007).

\section{Global Messages Received from the Project}

This metatheme captures discussions of global, overarching messages that key informant participants' received from the project. There were two specific themes of global messages from key informant participants and are described in detail below. Key informants were asked about 
whether they received any global, overarching messages from the project and what these might be.

Community messages. This theme captures key informant participants' discussions of global, overarching messages that they received from the project that relate to community and bringing people together. Participants expressed that they received community specific messages from the project. In particular, one participant explained that she found the project to be a good example of how to incorporate graduate work into the community and as a way to bring people together:

I mean, I hadn't read any theoretical stuff for what you did, but someone said it was really good and I think it was a really good example of how we can incorporate graduate work into our community and actually have it be a community thing. I think even some of our students got to know each other through your activities so that was nice. I've also noticed that with our first and second year [students] there is more of a community social atmosphere happening in that room than there was before and I don't know how much of that was the activity or other things as well...But having students initiating things and pulling them together makes a difference over us [RASS] hosting events all the time (HL).

The participant begins by providing a caveat to her response (i.e., she hadn't read the theoretical aspect of the project), but explained that her global message from the project was the community that the project built. Specifically, relationships were built through this project that spanned above and beyond relationships between the principal investigator (CJD) and the participants. In fact, participants formed and/or strengthened their relationships with one another. In addition to community cohesion, the project promoted an understanding among the community of ways that graduate work could be incorporated into the community, which can also be interpreted as capacity building. Specifically, community members can use the capacity they now have around incorporating graduate work to harness the contributions of Aboriginal 
graduate students in the future. This can further build relationships among community members and strengthen community cohesion.

Another participant spoke about her global message from the project, which was that the project would impact and teach those who haven't come to the University yet and how the project would impact community members who are yet to be present:

Or even, maybe it's not the exact same students, but the students behind them. A lot of what we do is for the faces who are not here yet. When we say that traditionally, as [noted her Nation here] people we mean the babies that have not yet come. When we think about it in an academic space, the faces not here yet are just supposed to not have come to school yet, the ones behind them. That is who you are doing this for because there is going to be a next year's class and those students will also be going through the struggles (GR).

This participant draws attention to the relational component of Aboriginal culture and how, community members that participated in the study will not be the only ones affected by it, rather, the students who come after them will also be affected, since the impacts can be farreaching. A report will be drafted and given to the partnering community (i.e., RASS) and, therefore, these findings can be used to inform a Wellbeing Activity in the future, other program development or even the ways in which the University understands Aboriginal post-secondary students and their mental health concerns. She used a traditional teaching to communicate her overarching project message (i.e., "...what we do is for the faces who are not here yet"), which helped her to articulate the impact of the project from her perspective.

Cultural revitalization/self-determination. This theme captures key informant participants' discussions of the community's need for cultural revitalization as well as selfdetermination and how this project began to address this need. Two participants spoke about their overall messages from the project as being related to cultural revitalization in that 
knowledge needs to come from the community and reflected back to the community, as this will promote revitalization and, eventually, self-determination.

That knowledge production needs to be back in the hands of the people who need it. It's not beneficial if it's just academic. It has to come from them, it has to return from them, and then it has to come from them again, and return to them again...Y You're not done with this project because you're going to return it back to them again and then you are probably going to find a way to have students voice go out. I know that you are going to use this as a spring-board and that to me is really important because it is going to be a demonstration for our students that participated and for those that you are going to inspire to realize the importance of the principles of OCAP, because that's what you are showing here. When I say you are going to take this away and re-vamp it, that's just you being a good Aboriginal researcher and knowing that you don't own or possess the research. You want to always return it. I know that is due to your training but it is also an internal part of you, which is good... (GR).

This participant draws attention to the importance of knowledge production as well as translation occurring within the community. She expresses an expectation of the principal investigator (CJD) taking the project further and using the project as a starting point (i.e., a 'springboard'). She stresses the importance of the principal investigator (CJD) continuing with the project and communicating the results of the project to the community, as this will model OCAP principles to other students, so that they can learn how to conduct culturally safe research within an Aboriginal community. She demonstrates her trust in the principal investigator (CJD) as a person (i.e., '...it is an internal part of you, which is good...'), the principal investigator's (CJD) use of OCAP, and her intentions to take the project to a place that will continue to benefit the community.

Another key informant discussed the importance of reflecting back the messages from community members that were provided to the principal investigator (CJD) during data collection and how this was a key overarching message for her from the project:

I think you are reflecting back and clearly illustrating the important words and messages about who we are. The identities are key in everything we do, the impact of where we belong and who we come from needs to be paid attention to, and that you use language 
about wellbeing and balance, and understanding that you are addressing the whole person (MK).

This participant demonstrates her trust in the principal investigator (CJD) and her expectation in the principal investigator (CJD) to clearly reflect the words and messages of community members' contributions to the project. Both of the quotes above are in line with the KT recommendations put forth by Hanson and Smylie (2006) who state that knowledge needs to come from the community and should be translated to the community, by community members using their language (e.g., 'wellbeing'). They also tap into the idea of cultural revitalization because both quotes speak to the need for Aboriginal knowledge production and for this knowledge to be given back to the communities, as this will be a form of cultural revitalization. The former quote draws attention to the OCAP principles, which has been outlined above, but briefly, it emphasizes Aboriginal people's right to own and control research that has been conducted with their community members and taps into the concept of self-determination, as has been defined above. Aboriginal communities have been working to take back their knowledge and control of their own destiny to achieve self-determination, as was the case prior to colonization (Asch, 2002). Both cultural revitalization and self-determination can be conceptualized as impacting as well as progressing one another in a forward direction.

\section{Present/Future of Mental Health Knowledge among Aboriginal Students}

This metatheme captures key informant participants' discussions of the present and future state of mental health knowledge among Aboriginal post-secondary students, which fell into three themes that are outlined in detail below. Key informants were asked about the present and future state of Aboriginal students' mental health knowledge and their responses are captured below. 
Stigma remains and normalization is needed. This theme captures key informant participants' discussions of the need for normalizing mental health in order to decrease stigma, since this is still a significant issue within an Aboriginal context and contributes to difficulties in the area of mental health and wellbeing. All five participants spoke about stigma as continuing to exist around mental health and how this is something that needs to change in the future.

Specifically, participants spoke about how mental health language can be stigmatizing:

...I'm coming from a place to advocate for students and often that's [using mental health language] the only way to get them what they need in terms of space and accommodations. I do feel reluctant at times [to use that language] because I do feel it tends to individualize students from the community. Particularly our students, I'm aware that people might start to see a trend in the Aboriginal community of students (HL).

This quote begins by positioning this participant as an advocate for Aboriginal students and she goes on to talk about her limitations in this area as related to the system. Specifically, using 'mental health' language is the only way that she can get students what they need in a Western institution that doesn't often recognize or acknowledge Aboriginal worldviews. Therefore, if she used less stigmatizing and more culturally safe language, students with whom she works would not be able to access necessary resources. She reports feelings of reluctance around using 'mental health' language, as she fears that such language further stigmatizes Aboriginal students and may isolate students from their community. Her reluctance in using mental health language demonstrates how her advocacy for students is in tension with the "rules" of Western resources acquisition. This is in line with discussions from the first phase of the project, as students spoke about intersecting stigmas, specifically, the stigma of being Aboriginal and struggling with mental health compounds with one another to create even more stigma and distress with which they will attempt to cope.

I think it [mental health] becomes the Aboriginal student's experience. All students experience this [mental health] but our students show it in different ways because of this 
and this and this. And so I'm worried about marginalizing our community further or marginalizing or individualizing people and making them feel like they don't belong even more because of that [mental health language] (HL)

This quote further demonstrates this difficulty (i.e., '...becomes the Aboriginal student's experience...') and speaks to the normalization of mental health while, in the same breath, noting mental health stigma. The two quotes above speak to the concern of having 'mental health' become specifically linked to Aboriginal students and how it may individualize and isolate people from their community, as people may begin to see mental health as specific to this population, further marginalizing Aboriginal students due to the stigma that surrounds mental health.

Participants spoke about how mental health is not talked about because of the shame around it:

I mean, we don't talk about mental health issues for a reason and it's because we are ashamed because of colonization. We used to think of mental health 'issues' as gifts from the creator, because it meant that people fought and saw things differently. Now it's stigma and...not addressed in wholistic ways, it's addressed siloed (GR).

This quote draws attention to the shift from an Aboriginal worldview to a Western worldview on mental health through colonization and how mental health is now targeted in a positivist Western fashion: as separate from the other aspects of the self (i.e., 'siloed'). This participant expresses desire to return to traditional ways of viewing mental health, which are considered healthier for Aboriginal people than current and Western perspectives. She also brings attention to the shame associated with mental health, which she positions as a result of colonization, rather than the mere existence of mental health.

The same participant expressed the fear related to mental health, regardless of how common it is: "I think that students are still afraid, even though so many of them have people in their families and in their lives that really struggle" (GR). This quote positions mental health as 
being stigmatizing. She noted the frequent nature of mental health issues; however, due to the stigma around mental health, students are afraid to talk about it or reach out for help, which again is in contrast to traditional Aboriginal perspectives on mental health and wellbeing. There is an indirect push for cultural revitalization within both of the quotes above, as they both call for a return to traditional understandings of mental health and wellbeing.

Although many participants spoke about their current frustration with regard to the stigma that presently surrounds mental health, many had hopes and ideas for the future regarding how to combat such stigma and promote normalization: "It is something that is not to be shamed and that at some point we will all need helpers. As helpers, we will always need helpers, but I hope other people will become much more open to getting the help they need". This quote demonstrates hope for the future in moving the understanding of mental health back toward the Aboriginal perspectives.

Participants specifically spoke about the need for increased knowledge regarding mental health in order to combat stigma and normalize mental health: "First of all, that the average person needs to know more about mental health issues to remove it from the stereotypical perceptions of mental health and moving in toward a more normalizing event that can happen to anyone." This participant is suggesting that the more we know about mental health, the less stigma that will surround it and also suggests that we need to move toward an Aboriginal understanding of mental health (i.e., it is normal), which is in line with other quotes pushing for a return to the traditional way of understanding mental health and wellbeing.

The quote below also tapped into the idea of gaining a better understanding of mental health, which will decrease stigma. She brought attention to institutions being uninformed, which 
contributes to the perpetuation of mental health stigma, while noting that through normalization, the fear related to mental health can be decreased:

I think we are at a very uninformed place within the University. I think there is still stigma around recognizing or saying that I have mental health stuff. I wish it was different because true mental health has become so synonymous that like... It needs to be changed to something more reflective of what's really going on. I think we are headed...I see the University in a lot of things it is doing, talking more about mental health and the normalization of it and taking away the scare factor so that people are becoming interested in understanding it. The more that people share, the more it's normalized because every family has at least one member who deals with mental health issues because it tends to run down through families. So it's far more prevalent in all cultures than most people recognize (FC).

This participant draws attention to the need for gaining a better understanding of mental health, what the University is already doing to promote this understanding, and the need for people to share information about mental health in order to normalize the experience of mental health issues, thereby decreasing its stigma. This again demonstrates the push toward recognizing and acknowledging the Aboriginal perspective on mental health and wellbeing (i.e., it is normal and it is part of everyone).

One participant had a specific idea of how to move people toward normalization of mental health rather than continue to stigmatize it:

Or just become part of the orientations [in the University]. Having mental health as part of the orientations when you walk in, it's an expectation that there's no stigmatization. We have an expectation at RASS regarding queer or racist comments: They're not said. Things like saying, 'that's crazy' need to be erased. That stigmatization of mental health really needs to be part of that foundation. However, I don't think it was thought about as deeply as it has been for other things, but whether it continues...(HL).

This participant suggests that there be a section on mental health during every student's orientation to the University and that they be taught how to be sensitive to mental health, perhaps by refraining from using particular language (e.g., 'that's crazy'). This may help remove the stigma around mental health and help move people's understanding toward normalization. 
In sum, participants agreed that mental health stigma is still present within the University, but participants expressed hope for the future and ideas regarding ways to normalize mental health and decrease the stigma. A common thread throughout these quotes is a need and want for mental health to be recognized and understood from an Aboriginal perspective, as mental health stigma did not exist within an Aboriginal context, traditionally. Although 'cultural revitalization' was not explicitly mentioned in any of the quotes above, many of the exerts can be interpreted as pushing for this.

Resources are needed. This theme captured key informant participants' discussions of the need for mental health resources to address mental health issues among Aboriginal people. Participants spoke about the current state of mental health resources, what is needed, and their hope for the future regarding such resources. One participant spoke about what he would like to see regarding resources for Aboriginal students and what currently exists:

I mean, I would love to see resources in our community, particularly for Aboriginal students to access. I think a lot of the resources for Aboriginal students to access are very addictions focused and are focused on things like homelessness and unstable housing and stuff. That may not be students' primary concern or they may not feel that they fit in those service offerings. But I also feel that Aboriginal students don't really get what they need from campus mental health services either. Because there is an element of their cultural experience and their historical experience that they carry and that their families carry that doesn't exactly translate to mainstream services. So, one thing I would like to see grow and develop are services for Aboriginal students where they can meet with people who have that knowledge or expertise, including things like academic assessments as well as mental health disabilities (SE).

This participant identifies a resource gap within the University, including gaps in assessments for learning and mental health disabilities as well as a general lack of understanding within the University regarding their cultural and historical experience and students need to be able to access culturally safe services within the University. This quote is in line with discussions from the first phase of the project, which emphasized the clash between Aboriginal and Western 
models of mental health. Specifically, that fact that historical experiences of Aboriginal people are rarely acknowledged within the mainstream system and there is a need for Aboriginal students to meet with service providers who have expertise in Aboriginal culture and worldviews.

Other participants spoke about language as a means through which Aboriginal students can access mental health services: "In some ways educating students on mental health is an advocacy piece because it teaches them where their rights are and where their supports can be..." This again taps into a theme that was found in phase two of this project, and has been mentioned previously during the current phase, emphasizing the need for students to be able to advocate for themselves, especially in the area of resource access. This same participant spoke about mental health language as specifically helping Aboriginal students access resources:

One of the beauties of using mental health language is it gives them access to services and accommodations that they wouldn't otherwise. So, in some ways I like to talk about 'wellbeing', and other ways there is a real need to talk about it in terms of 'mental health'. Talking about it in terms of mental health does give them access to accommodations and disability services and things that will help them be successful at the University level (HL).

This participant draws attention to the importance of language and how it can be a major source of advocacy with regard to accessing resources. She highlighted the conflict between the language she would prefer to use (i.e., language that is consistent with Aboriginal worldviews) and the language that she needs to use in order to connect students with necessary resources, which again taps into the clash of Aboriginal and Western knowledge systems that was emphasized in the first and second phases of the current study. The mainstream system has rules and language associated with these rules that stem from a Western worldview, which is often invalidating as well as limiting for resource access among Aboriginal people. 
Another participant spoke about dialogue as an important component of accessing resources:

Dialogue is important when you come to the issue of mental health, not only for the person that is affected but also for the family. That's what is hard. Mental health does affect the whole family and the community as well. So, dialogue is important because it creates support systems that can open doors (SE).

This participant draws attention to the wholistic nature of mental health (i.e., it involves one's family and community) as well as the importance of communicating about one's mental health struggles to one's support system, as this will open doors to such resources, whether it be family-based, community-based or service-based.

One participant spoke about RASS as a current resource for Aboriginal students and how it can be helpful for those with mental health issues (i.e., it supports students, wholistically), but that those who are not connected to RASS, or any other place of support that promotes a sense of community, may be struggling without a community to fall back on and may feel as though there is only one model of mental health (i.e., the positivist Western model):

...For the folks who have connected with RASS, I think that we've made some good progress around people taking responsibility for and not being ashamed of talking about their mental health. I think that for the folks who haven't connected with RASS, and because our primary focus is people who are new to Ryerson or transfer students and our access students, it's the folks in second, third or fourth year or even graduate programs who may very well be connected to a strong vibrant community, but my thoughts go to the people who are not connected and may be struggling to find places of support. Or they may be caught in the belief that current models are the only models available to them (MK).

This participant draws attention to the need for community around mental health difficulties and how RASS provides this for students at Ryerson University. She also discusses RASS as specifically tending to student's mental health concerns below:

...I also think that one of the things that RASS already does as part of its existence is tend to the mental health of students. So, I think it is a much safer place for Aboriginal 
students to talk about mental health issues because there would be no judgment. It is part of what we provide, right? (MK)

The same participant notes the creation of a safe place for Aboriginal students at Ryerson University, since RASS works to create a non-judgmental atmosphere for students. She positions this as a responsibility of RASS (i.e., It is part of what we provide..."). She goes on to discuss her pride in different relationships that RASS has formed with other services:

I'm proud of the collaborative work that we've [RASS] done with the health promotions and peer support in the past. I'm proud of the working relationship that we have with [medical physician] who heads up Ryerson's mental health working group. I believe we have a strong ally in her (MK).

This quote highlights the collaboration that RASS has achieved with health promotion and the allies that they have made in order to support students in the best way possible. She speaks to the relationships that RASS has created with other services including health promotion and the Mental Health Working Group, which aims to respond to the impact of mental health issues on students, staff and faculty at Ryerson University with a strategy for addressing this impact (Ryerson University, 2013). The three quotes above tap into the emphasis on relationships and community, as this plays a large role in creating a safe space for Aboriginal students to access (i.e., RASS) and create more resources for students through collaborating with other professionals.

This same participant spoke about how this project could impact resource development at the University in the future and how she could have used the project differently in order to facilitate this:

...In hindsight I think it would have been helpful for another of the staff to attend [the Wellbeing Activity] just so we might look at how we could build that into our programming, how we could engage or partner with our own health center to get them rethinking wellbeing, balance, etc. The other thought that I had was how to get the academic program to understand that they are one of the principle sources of providing resource people. They need to think about understanding the Aboriginal community, as 
you've heard with our community on campus, the way to engage us needs to happen differently. The models being utilized don't quite hit the mark. I think the segment of you of having RASS as a partner is really an opportunity to say, 'we don't want to go unnoticed'. You are providing some critical information that should be paid attention to by the academic program (MK).

This participant specifically highlights the need for the University to recognize Aboriginal worldviews, in particular, regarding mental health and wellbeing and that the services offered at the University 'don't quite hit the mark' for Aboriginal students, especially since they are often coming from a different worldview, a different historical lens, as well as there being a general lack of coordination of services for Aboriginal students. She emphasizes the fact that the project presents an opportunity for Aboriginal worldviews in the area of mental health to finally be acknowledged by the University, especially through the project's partnership with RASS. This again promotes the idea that Aboriginal people are emphasizing a movement toward revitalizing Aboriginal perspectives within both the Aboriginal and non-Aboriginal community.

Participants expressed their hope for the future around mental health resources for Aboriginal students:

I think you if you wanted to put an addendum to your report, where do the caregivers go to keep them focused? There should be a program about who cares for the caregivers. There should be somewhere they can go to take care of themselves. A caregiver is no good if they don't care for themselves (SE).

The participant above emphasizes the need for resources for healers and caregivers so that they can better support Aboriginal community members. This is in line with a phase one discussion on how those helping and being helped are not considered to be mutually exclusive from an Aboriginal perspective. Rather, helpers will require resources and those accessing help may be called upon to help others depending on community needs at various points in time. 
The following quotes note the importance of incorporating resources for mental health during students' orientations to the University and hope for people to be able to get the resources they need in the future:

I would like to see it where it comes through a recruitment office [of the University] or it comes through orientation that it become a pamphlet that is handed out to students so that right away, right off the hop, there is a normalization of it. These are our mental health services. There are all kinds of ways to get a very positive message across in a very small package of a pamphlet, where a student would know where to go to seek assistance (FC).

It [mental health] is something that is not to be shamed and that at some point we will all need helpers. As helpers, we will always need helpers, but I hope other people will become much more open to getting the help they need (FC).

These two quotes above further emphasize the importance of communicating mental health as a normal phenomenon and through such normalization, students will be more likely to advocate for the help that they might require. These ideas, coupled with the KT recommendations with an Aboriginal context (e.g., Hanson \& Smylie, 2006), could create an effective knowledge translation strategy for the University to provide resources and services to Aboriginal students.

Indigenizing institutions are needed. This theme captures key informant participants' discussions of the need for indigenizing institutions in order to address the current issue of mental health and wellbeing among Aboriginal post-secondary students. Participants explicitly talked about the need to focus on spirituality and Aboriginal perspectives within various institutions and the barriers faced in trying to move in this direction. One participant, in particular explained the need to focus on spirituality and wellbeing in the classroom and beyond. He talked about how institutions have not yet grasped the importance of spirituality for Aboriginal people.

...we've covered the mental to a certain extent, but it's just one facet of it and that's the classroom, the mentality and how we process that sort of thing, but we have not looked at 
the overall wholistic mental wellbeing of the student outside the four walls of this institution. I think we need to improve on that because what affects them out there will affect them in here eventually. So that's one idea. I think we need to re-focus on the emotional, in the sense that we can tweak it so that it exists within their studies.

Physically, I think we are doing our best with that. Spiritually, we could do more work on that. We talk a good game. It sounds good to talk about these caregiving abilities but the spiritual aspect of it, I don't think there is enough within the institution...It's unfortunate that they [institutions] haven't grasped the importance of our spirituality. I think once they do that, the students will be more successful (SE).

This participant explains that through increasing spirituality within institutions and within the education system, specifically, Aboriginal students' wellbeing could be increased. This quote taps into the importance of having the Aboriginal perspective acknowledged within institutions, as Aboriginal students will benefit greatly from this. The quote draws attention to the common clash between Aboriginal and positivist Western worldviews and how Aboriginal worldviews are often invalidated within mainstream institutions, which can further contribute difficulties with mental health.

This same participant spoke about the struggle he faced when trying to incorporate spirituality and Aboriginal worldviews into the University:

With RASS, I wanted to bring the sweat lodge in, but the areas to make it available to the students...I was going to do all the paperwork but then I thought it's not worth it because we as a people should have that right to make that decision and not have to go through the obstacles the University gives us. Liability was a big one. We know we have systems in place to take care of that [liability]. I think their [the University] motive is always the financial aspect of it and I could see people buying into that and I just said, "no". The students were disappointed but I made that decision on the sense that it was taking away our sense of being the original people of this continent and we had to conform to these people. That is what I think is mentally distressing sometimes. I don't want our students to fully conform, but I also realize its importance in getting a degree, they have to do some things. But it should not be a complete sacrifice, to sacrifice your Indigenous values. I felt for the students because so many wanted it [sweat lodge]. If we had it our way, we would have had them...I wanted to bring it in for the students and I thought it would help with the mental distress. It would have helped alleviate that (SE).

The above quote positions the system is not organized in a way that supports the inclusion of spirituality. In particular, this participant wasn't able to follow through with a sweat 
lodge ceremony due to system-level barriers and notes the common necessity of Aboriginal students having to 'conform' to the dominant culture and sacrifice the Aboriginal way, which is not helpful for Aboriginal students' wellbeing. This participant also describes his resistance against the system, as he decided that he should not have to justify traditional ceremonies to people who would not and could not understand their importance or trust that Aboriginal people have their own processes in place to protect their community members.

This same participant explained that Aboriginal students exist in a system that doesn't recognize who they are, which needs to change:

...I've talked to Native kids and let me tell you, I have a lot of respect for the Indigenous kids. First of all, sometimes they are in a system that doesn't recognize who they are, which creates great mental distress as they grow up. Secondly, the importance of their background is not really explained. That will affect them in terms of mental distress at some point. But now they are trying to change that... There is a course now where Indigenous people are at the forefront, residential schools are talked about. I went to do a presentation about residential schools to a grade four class. So, they're starting to appreciate the fact that what transpired with the Aboriginal history has contributed to a lot of the mental health issues we have today...I'll tell you, the education system, for the longest time, did not emphasize our existence and in the proper context and that does create mental stress. From there they graduated to other issues like negativity, addictions (SE).

The quote above specifically links Aboriginal identity and acknowledgment of its existence to wellbeing (e.g., '...doesn't recognize who they are, which creates great mental distress...'), but he explains that he has faith in the changes that he sees coming, as related to the recognition of Aboriginal people. This quote emphasizes the need for institutions, especially educational institutions, to inform themselves on Aboriginal history, culture, and identity, as they are presently continuing to traumatize Aboriginal students simply because of the ignorance that permeates throughout these institutions.

This participant also spoke about the changes being made to the education system, specifically, how Elders are now being included in this system. He noted how important it is to 
have dialogue within the school system regarding the history of Aboriginal people, which is sometimes met with frustration among Aboriginal students:

Working with TDSB I go to all levels: I go to the Trustees, I go to the Senior Administrators, and I go to Teachers and Principals. Most importantly I'm reaching into those little seeds who are human beings. If it was left up to me I would do presentations to children all the time, but I do high school. When you do high school they've already formalized their thinking and sometimes that thinking is not proper. For example, I did a presentation the other day and the students got upset at what happened in this country. They couldn't understand why a country would do this to its original people. It got to the point where one of the students started using foul language because that's how she could express herself. It was great because it creates dialogue, and that's important (SE).

This participant draws attention to the importance of targeting children to prevent mental health struggles. He explained how he is currently becoming involved in the education system, which is progress, since he identifies as being Aboriginal and can influence change from this perspective. This demonstrates some steps that are being taken by the school board to acknowledge Aboriginal history and people within the school system. This quote demonstrates the fact that cultural revitalization is in forward momentum at present.

This participant continued to speak about the changes he sees coming within the education system:

It's going to happen too! That's the beauty of it. So, I think your dissertation is something that really needs to be looked at. It needs to expand. It's ironic that we are talking today because I was just invited to this think tank, that's the medical school and they were saying how do we reach [Aboriginal people]? I said, 'well, when people talk about medical school they're thinking about the body and not about the mental aspect, that's medical. We don't think about how the emotional and spiritual can help in the process'. If you are one day sitting in a position to help people I hope and believe you should be able to say, 'here are some of our cultural beliefs'. Then all of a sudden you have that wholistic approach (SE).

This participant expresses hope for the future and his ability to influence the education system from an Aboriginal perspective presently, as well as in the future. He explains his involvement with various institutions and being invited to speak at 'think tanks' within a medical 
school, where he is able to influence students to think about their field from a wholistic perspective. This participant seems hopeful of the future and of the progress that has already occurred in Indigenizing various institutions. He also expresses an expectation of the principal investigator (CJD) in expanding upon the work that has already been done for the project.

In addition to the quotes above, another participant drew attention to the importance of incorporating Aboriginal worldviews into the education system:

My hope is that as we continue to de-colonize, if I think back even five to ten years, my own understanding of being Aboriginal was different than it is today. As we de-colonize as communities and we get healthier, I hope that we can return to an understanding of mental health being a continuum that we all fluctuate on, and that having issues with mental health is normal (GR).

This participant reports her hopes above in moving the understanding of mental health toward an Aboriginal perspective, and in doing so, healthy communities are promoted. This participant also emphasizes the link between the recognition of Aboriginal worldviews and perspectives on wellbeing. This quote is in line with cultural revitalization and how moving toward and traditional Aboriginal understanding of mental health within the system can promote significant changes in Aboriginal people's wellbeing.

Another participant spoke about the importance of this research project in moving Aboriginal perspectives forward within the system:

Just ensuring that it's [this project] not a one-time event because you were here and this was your research. I really want them to understand that again, if we take a look at the human resources center available and the types of programs and services available to us as Indigenous, Aboriginal people, particularly in psychology and the ways in which they provide support, need to take into account what our community members are saying about how they would like to be engaged and the types of support that are critical and that you were able to create something that was tailored to the folks that you engaged (SE).

The above quote expresses an expectation of the principal investigator (CJD) to take the project further, specifically, to use it as a teaching tool for the University and for services within 
the University. She highlights the lack of acknowledgement of Aboriginal perspectives and worldviews, which is a common underlying thread of what many participants spoke about throughout this entire project. This same participant provides insight regarding strategies to promote indigenous knowledge into the educational system:

So there are a couple of things I would like to do. One is when we have that report, we launch it. I would like to invite our ministry partners to come and hear about this and to engage our health center, invite the academic program to participate. Let's create an opportunity to act on this [project] (MK).

This participant suggests specific ways to move the Indigenous perspective into the University institution. She emphasized the importance of the report, which the principal investigator (CJD) plans to provide to RASS. This further demonstrates her expectation of this project to continue and to create change over and above the purpose of a dissertation, which aligns with the aims of the present study to be conducted from a community-based approach. This same participant emphasized the opportunities that the current project can provide in creating changes within the University:

She [health provider at Ryerson] has a solid foundation, when we talk about Aboriginal mental health. That work, the definition [of mental health] needs to come from our community here. I think that we have a very good opportunity, because you made this your focus for a thesis that we can reflect it back to the academic program. There are going to be other students to come and you have been very clear about what our community expects and how they would like to be engaged around mental health. How can we take the opportunity and begin to see some changes in the academic program? (MK)

This participant guides the principal investigator (CJD) in her responsibility to reflect this knowledge back to the institution and to ensure that there is clarity around exactly what the Aboriginal community expects regarding mental health services. This expectation around the principal investigator's (CJD) responsibility is in line with conducting research in an Aboriginal context, as relationships and work do not end once the data is collected, rather, they continue, as 
there is more work and opportunity that will stem from this project even after it is complete (Baskin, 2005). In conducting this project, the principal investigator (CJD) has assumed a responsibility in ensuring that the voices of this community are heard and acknowledged. The dialogue around this has continued and is beyond the scope of this dissertation.

This same participant expressed her frustration with the fact that many Aboriginal people across the country are talking about mental health and wellbeing from an Aboriginal perspective, but that the system does not recognize them because they don't have the 'right' credentials:

...I believe there are a number of people currently doing that work [in mental health] and aren't acknowledged for the work they are doing because they don't have the certification behind them. I think about [Elder] who does traditional counselling, or I think about the healing lodges across the country that utilize Aboriginal philosophy and methodologies (MK).

The above quote further demonstrates the importance of incorporating Aboriginal perspectives into the current system, especially the education system, and how many people are talking about mental health from an Aboriginal perspective, yet this knowledge is rarely recognized if they are not formally educated in this area, which also needs to be considered and changed. This in line with common discussions throughout these results on the topic of a clash between Aboriginal and Western worldviews and, specifically, how Aboriginal worldviews are commonly invalidated within the mainstream system.

\section{Project Impacts on Key Informants}

This metatheme captures spontaneous discussions of ways in which the project impacted key informant participants either mentally, emotionally, physically, or spiritually. Key informant participants were not asked questions about how the project impacted them; however, upon reviewing the transcripts, this metatheme emerged. In particular, four participants spoke throughout the interview about various ways that the project impacted them. Although this 
project was designed to impact Aboriginal students, connections between and among people and their experiences were far-reaching, as is in line with an Aboriginal worldview. Therefore, it is not surprising that those involved in helping the project come to fruition would learn important lessons for themselves.

You know it's been a long process, as a $\mathrm{PhD}$ dissertation is, but it's actually taught me...I'll tell you the truth. Your dedication to your studies has taught me that in some ways I need to do a $\mathrm{PhD}$ as well...The process has given me a better understanding of expectations of what a $\mathrm{PhD}$ is, but also really flexible. I find that you've been open to your learning and how things might need to flow differently. You've had a lot of things put up in front of you, especially with traditional people. And there have been a lot of traditional people throughout this whole thing. You haven't treated that as a disruption, you've just accepted. It shows your determination and your strength. I've actually enjoyed watching you do this process (GR).

The quote above demonstrates how the project, and the relationships that came from the project, inspired one key informant to pursue her $\mathrm{PhD}$. This is an interesting result, since the project was designed to provide resources to help students manage their own mental health and wellbeing, with the ultimate aim of fostering their educational progression. Through the process of implementing this project, a key informant and staff member, who was not targeted by the project, was inspired to pursue her educational goals. This is evidence of the strength of Aboriginal knowledge systems in emphasizing relationships and interconnectedness between all things. Another participant outlined the ways that he was impacted by the project:

What I take away is one of the most important values is an appreciation of the students, like yourself, and the others from my office. For me that was the ultimate success of it and it will be forever etched in my existence, a project like yours (SE).

This quote shows how the project reminded him of how important the students are and increased his appreciation of them. He continued to express the impact of the project on him through the quote below:

In a way it did affect me, meeting you. I tell people about you. I have a student who is working on her degree process. I said, you should meet this young lady because she's 
doing something on this area of mental health in the wholistic sense and not just the illness part but looking at a broader more effective way. I said if you had a chance to meet her I think it would give you the motivation to see the importance of what you do. She agreed (SE).

This quote shows how he plans to connect other people to the principal investigator (CJD) of this project to continue to impact more community members through this project. Another participant simply stated, "I just hope this project is...I know there is a marking system to it, but it's so much more. It's life altering" (GR). This participant is noting that the project will be evaluated, since it is a dissertation, but also that the impact of it was even more meaningful than the completion of the principal investigator's (CJD) degree, as it personally impacted many people involved, including the principal investigator (CJD). It is interesting that the KT development, implementation and evaluation was meant to impact students and their wellbeing, but in fact it went further than that, and impacted staff involved as well as the principal investigator (CJD) conducting the study, in very significant ways.

The key informants' interviews provided insight regarding how the project impacted and taught Aboriginal students about mental health knowledge and resources, acceptability and changes needed with regards to the research process, community needs that were addressed through the project, opinions on the present state of mental health knowledge and ideas for its future, and ways the project impacted key informants in addition to Aboriginal students. This phase provided evidence for the implementation of effective community engagement over the course of this project, which is an expectation of community-based research, and provided new ideas regarding mental health topics to be addressed through knowledge translation strategies in the future. 


\section{Discussion}

This project aimed to develop, implement and evaluate a knowledge translation activity using a community-based approach in order to address mental health barriers that may prevent Aboriginal students from achieving their educational goals. Education has been described as 'our buffalo' by Aboriginal nations (Alberta Education, 2002) and is, therefore, seen as a way for Aboriginal communities to move toward self-determination (Alberta Education, 2002). The main findings of this project are summarized and discussed below. Many of the findings stem from a social constructionist lens, which assumes that the categories through which we understand the world do not necessarily represent real divisions, the way we understand the world stems from historical and cultural contexts, and understanding develops through social interaction (language in particular) and action.

\section{Tool Development Phase}

In the Tool Development Phase, mental health was defined from an Aboriginal perspective and a KT activity was developed. Mental health from an Aboriginal worldview was described as inseparable from the self, a community responsibility, being passed on intergenerationally (i.e., being passed on from generation to generation), as being central to one's identity, as being closely tied to stigma, as being impacted by cultural connection, and as being impacted by a clash between Aboriginal and Western perspectives. This definition is very much in line with the tenets of critical psychology, as it challenges mainstream psychology's definitions of mental health and is in line with social constructionism, since this definition challenges the idea that essences exist, which invariably define "mental health". This is demonstrated by the fact that a definition of mental health, as constructed by Aboriginal postsecondary students and staff is quite different from a mainstream definition of mental health 
presented above (American Psychiatric Association, 2000). More specifically, Aboriginal students and staff spoke about how balance is sought after, but an illusory concept, since it can never be achieved; however, mainstream concepts of mental health assume that people have control and self-efficacy over their mental health and wellbeing (Hepburn \& Jackson). There is also an assumption from an Aboriginal worldview that the helped and helper are not mutually exclusive, as is assumed within mainstream psychology. Aboriginal participants drew attention to community members alternating between helpers and those needing help. In addition, and in line with the frequent emphasis on relationships throughout this phase, participants spoke about the importance of establishing relationships with their helper. Although the therapeutic relationship is considered to be an important component of any intervention sought in mainstream psychology (Horvath \& Lester, 1993; Krupnick et al., 1996; Martin, Garske, \& Davis, 2000; Vasquez, 2007), there are limitations around disclosure and boundaries (American Psychological Association, 1992; Epstein, 1994; Goldstein, 1994; Sinclair \& Pettifor, 2001). Although these boundaries exist within an Aboriginal context, there are different expectations around them. Specifically, Aboriginal participants noted that it is important for their helper to disclose personal information, especially if they have experienced similar struggles to those seeking help, and it is important to see their helper as being on equal ground as them, facilitating client comfort within the therapy context. This is again in contrast to expectations within mainstream psychology (American Psychological Association, 1992; Epstein, 1994; Goldstein, 1994; Sinclair \& Pettifor, 2001) and, therefore, challenges mainstream assumptions.

In addition to the definition of mental health found in this phase, challenging traditional perspectives of mental health within psychology, the definition is very much in line with how the concept is discussed within the literature examining mental health from an Aboriginal 
worldview, as other authors have also challenged mainstream ideas in psychology. In particular, Aboriginal worldviews and culture emphasize wholism (Alberta Mental Health Board, 2006; Blackstock, 2008; Edge \& McCallum, n.d.) and, therefore, it is not surprising that mental health was seen as being inseparable from the self as a whole (i.e., inseparable from the physical, emotional, mental and spiritual aspects of the self). Smye (2001), the Aboriginal Healing Foundation (2003) and Isaak and Marchessault (2008) all describe mental health as being a wholistic concept. Vicary and Westerman (2004) specifically note that mental health cannot be separated from the self. This idea, in particular, is very different from the way in which mental health is defined within mainstream psychology (i.e., addressing particular symptoms; American Psychiatric Association, 2000). As well, mental health was described as a community responsibility, which aligns with the interconnectedness that is emphasized within Aboriginal culture (Smylie et al., 2003). From an Aboriginal perspective, we are all connected to one another and relationships are key (Baskin, 2005; Smylie et al., 2003), therefore, community members must support those who are suffering in various ways. Mental health has been described as resulting from disconnections to family, community, and the environment (Cain et al., 2011; National Aboriginal Healing Organization, 2009). Relationships and community are not frequently emphasized within mainstream psychology, but rather, the isolating nature of mental health was viewed in this project as the "Western way" rather than the Aboriginal way.

It is not surprising that intergenerational trauma was described as impacting mental health, since numerous authors have supported this idea (e.g., Batiste, 2000; Connors, 2007; Little Bear, 2001; McCormick, 2000). Mental health from an Aboriginal perspective was conceptualized as being impacted by intergenerational trauma. Related to intergenerational trauma, identity shame and struggle was highly emphasized among Aboriginal students. 
Emphasizing identity as impacting mental health also fits with literature stating that strengthening ethnocultural identity can improve mental health among Aboriginal people (Kirmayer, 2003). The concept of identity as related to mental health has been discussed within mainstream psychology through the works of Erik Erikson, in particular. Erikson (1970) discussed the concept of "identity crisis" (i.e., being partially conscious and unconscious, having a developmental period, existing in both the past and present, and encompassing both psychological and social components) as contributing to psychopathology and as being an important concept in the area of psychoanalysis (Erikson, 1970).

Stigma was found to be a significant theme as related to the concept of mental health in this phase of the study and has been discussed as a barrier to treatment within an Australian Indigenous context (Issacs et al., 2010). Stigma as a barrier is in line with discussions from Aboriginal students and staff, who noted that it is difficult for people to access treatment due to the stigma. Mental health stigma was discussed as something that is inconsistent with traditional Aboriginal culture, but exists within this culture due to colonization and the impact of mainstream worldviews. Traditionally, Aboriginal people viewed mental health as a normal part of the whole self, rather than something of which to be ashamed (Vicary \& Westerman, 2004). Participants in this phase pushed for a restoration of their traditional way of thinking about mental health and wellbeing. Cultural connection being tied to the concept of mental health is strongly supported in the current literature. In fact, the prevalence of suicide has been reported to be six times higher among Aboriginal youth compared to non-Aboriginal youth (World Health Organization, 2002); however, Chandler and Lalonde (2008) have demonstrated that the rate of suicide among Aboriginal youth varies substantially and such variation was found to depend on the cultural continuity within these communities. In particular, those communities that had more 
cultural continuity (i.e., had meaningful connections to their traditional past through the use of their traditional language, access to ceremonies and control over their systems) had lower suicide rates, suggesting an important relationship between the two. Within mainstream psychology, cultural connection is not recognized because all are assumed to come from the same or similar cultural background and differing cultural backgrounds or perspectives are not considered (Fox et al., 2008). Finally, the clash between Aboriginal and non-Aboriginal worldviews found in this phase is a common theme within the literature. This clash is also represented in the discussion above regarding the definition of mental health from an Aboriginal perspective and how different the themes found in this study are from positivist mainstream perspectives on mental health. It has been emphasized within the literature that mainstream services are often culturally unsafe for Aboriginal people due to the lack of validation and knowledge of Aboriginal perspectives and culture within these services. Because of this, there is a lack of culturally safe services for Aboriginal people to access (Assembly of First Nations, 2007). This idea can be taken further than the area of mental health, as this theme (i.e., a clash in perspectives) has been found in literature examining education (Baskin, 2011), healthcare (Assembly of First Nations, 2007), and politics (Idle No More, n.d.), to name a few.

The findings above regarding how mental health is conceptualized from an Aboriginal perspective fit with the present literature examining this concept, while challenging the status quo that is mainstream psychology. These results open the door for the recognition and acknowledgement of Aboriginal worldviews and perspectives within psychology. There are numerous implications of the above findings over and above its results. Firstly, it challenges the discipline of psychology to consider alternative ways of defining mental health. The mental health definition found in this phase can also be applied to various institutions where mental 
health is a prominent issue, including, clinical and educational settings, in particular. There are few mainstream clinical settings that recognize Aboriginal perspectives, especially those perspectives that clash with the way in which mainstream organizations often define mental health (i.e., being symptom-focused and using the Diagnostic Statistical Manual; American Psychiatric Association, 2000). However, Aboriginal students as well as non-student community members access mainstream services, in addition to culturally specific services (Vinkle, 2012) and, therefore, these findings may provide such programs with insight regarding how to validate these perspectives and, therefore, more effectively help Aboriginal people progress through any current struggles with mental health. These findings can be used as a training aid to provide mainstream mental health professionals with a guide regarding how to understand their Aboriginal clients and how to acknowledge the worldviews from which they may be coming. Increasing the effectiveness of mainstream mental health resources can aid Aboriginal people in accomplishing their education goals and work to remove mental health as a barrier to such achievements.

Furthermore, educational institutions and the services offered through these institutions, rarely acknowledge Aboriginal perspectives on mental health (Baskin, 2011; Battiste, 2000). As well, these institutions rarely acknowledge Aboriginal people and their identity, which further contributes to mental health concerns among this population (Bougie et al., 2003). The findings reported above can provide insight to help such institutions understand that Aboriginal people would like to be acknowledged within the educational system and ways that these institutions are currently operating that may in fact decrease an Aboriginal person's wellbeing. In particular, training of staff and faculty on the history of Aboriginal people; including Aboriginal contexts in the current curriculum; and working with students, staff and school services to normalize mental 
health across campuses could significantly aid in Aboriginal students' wellbeing, facilitating the completion of their education goals.

$\mathrm{KT}$ preferences were also found to challenge traditional perspectives on $\mathrm{KT}$ and were fitting with the current literature. Aboriginal students noted that they wanted Aboriginal perspectives of mental health to be emphasized throughout the KT activity, which is in line with the need and want for cultural connection and continuity that has been demonstrated to relate to wellness (Chandler \& Lalonde, 1998; Chandler \& Lalonde, 2008). As well, Aboriginal students and staff constructed the KT activity to cover the following topics: coping strategies, resources to access for mental health issues, understanding emotions and different mental health issues (e.g., anxiety), the mental health system, where to get mental health information and normalizing mental health. Connors (2007) notes that through colonization and historical trauma, often times, Aboriginal people have a lack of coping strategies available to them, since culture has been fragmented from such trauma, and without culture, coping is difficult. Therefore, it is not surprising that coping strategies and understanding one's emotions emerged as themes regarding content that students wanted covered during the KT activity. As well, Aboriginal people are a commonly underserved population with regard to resources (Jiwa et al., 2008) and, therefore, it is fitting with the current literature that Aboriginal students would want information on resources as well as information on how to navigate the mental health system.

Mental health stigma has been found to be a barrier for Aboriginal people to access services (Isaacs et al., 2010) and was discussed as compounding with the stigma of being Aboriginal. Therefore, it is fitting that students would want a discussion aimed at normalizing mental health during the activity as well as resources to help find accurate mental health information, which will decrease stigma through an increased understanding of the phenomenon. 
Students and staff also noted that they would prefer to move away from using the term 'mental health', but rather use the term 'wellbeing', due to the stigma that surrounds 'mental health'. This recommendation is in line with Kirmayer et al. (2003), Kirmayer et al. (1997), Smye (2001), Alberta Mental Health Board (2006), and Blackstock (2008), who all use terms such as 'wellness' and 'wellbeing' instead of mental health. The term 'wellbeing', not only diminishes the stigma that surrounds mental health, it also more accurately represents the way in which Aboriginal community members think about mental health and how it is defined from an Aboriginal perspective (e.g., Smye, 2001; Edge \& McCallum, no year; Kirmayer et al., 1997).

In changing the language of mental health, the shame that surrounds this concept is reduced. Shame is a common theme within literature examining intergenerational trauma across contexts. In particular, Japanese offspring of parents who had been incarcerated in internment camps during World War II were interviewed regarding how their parents' internment impacted their lives (Nagata, 1991, 1993, 1998). Many described their parents' experience as a family secret and described a sense of shame and inferiority about being Japanese. They also described a lack of confidence in their rights as Americans (60\% of those interviewed were American citizens; Nagata, 1991, 1993, 1998). Similarly, a study of incest survivors' offspring reported strong feelings of shame as impacted by the family sexual abuse (Fisher, 2006). Intergenerational trauma and shame are connected to each other in literature examining populations outside of an Aboriginal context. The core issue is regarding one's identity, since when shame in oneself is prominent, mental health, or anything contributing to struggle can compound that shame. Mendelssohn (2008) writes about the shameful reaction of Israeli society to the influx of Holocaust survivors after the war, as experienced in concentration camps, were not talked or asked about as this would be in contrast to the pride, self-confidence and self-idealization that is 
necessary to fight and win the War of Independence. This demonstrates a cycle of shame and self-abasement counteracted by pride of the self for defensive purposes (Mendelssohn, 2008). The stigma related to mental health within a mainstream context was reported as being adopted by Aboriginal participants in this study, who report simultaneously seeking cultural revitalization and building pride in their identity. Therefore, it is possible that a similar cycle is created within an Aboriginal context and, as a result, shame will likely be difficult to overcome, so stigma continues to impact the shame experience.

Students and staff discussed the method through which they would like to receive mental health information. They emphasized the importance of active participation and that a community Elder should co-facilitate the activity. These recommendations are in line with MacCaulay et al. (1997), who noted that information should be presented using active participation and that information should be delivered to community members, by community members (e.g., communicated by Elders). In addition, Hanson and Smylie (2006) emphasized active participation during KT in an Aboriginal context and McShane et al. (2006) suggested that community cohesion and receiving health information from community members is important in facilitating the process of information sharing. Participants also preferred that the activity be implemented in small groups, which is in line with Smylie et al. (2009) who found that First Nations people preferred individual communication due to stigma-related concerns. Small groups also promote more intimate interactions, which is in line with the community cohesion recommendation by MacCaulay et al. (1997) and McShane et al. (2006). Participants wanted information to be shared during the activity using storytelling, which is in line with Smylie et al. (2009) who state that experiential knowledge is highly valued when implementing a KT activity in an Aboriginal context. Participants stated that they wanted mental health information 
communicated to them using sharing circles, which is in line with Baskin (2005) who states that culture and traditions should not be compromised during the research process and that traditional methods (e.g., sharing circles) should be used to acknowledge the importance and legitimacy of this culture.

Aboriginal students' and staffs' construction of the KT activity fits with the definition of $\mathrm{KT}$ from an Aboriginal perspective and with recommendations for $\mathrm{KT}$ implementation within an Aboriginal context. Specifically, KT has been defined from an Aboriginal perspective as sharing what is known about living a good life (Kaplan-Myrth \& Smylie, 2006), which includes translating knowledge to community members such as students. This is different from mainstream KT methods, which mostly focus on translating knowledge to health care professionals (Government of Canada, 2014). The findings discussed above challenge traditional definitions of KT, which is fitting with the tenets of social constructionism, since challenging the status quo of traditional psychological terms and concepts is exactly what social constructionism aims to do (Burr, 2003; Gergen, 2009; Hacking, 1999; Jost \& Kruglamski, 2002).

There are important implications of the KT preferences found during this phase. These findings challenge the way in which $\mathrm{KT}$ is traditionally done by changing the focus from health care providers to community members, which is rarely emphasized in the current literature and could have significant benefits for, not only Aboriginal community members, but also the nonAboriginal public. These findings also have systemic implications. In particular, the finding that resources, and information on resources, as a need for Aboriginal students puts a spotlight on the gaps in services within the community and within educational institutions. In Toronto, Ontario, there are various Aboriginal specific services available (e.g., Anishnawbe Health Toronto, Native Child and Family Services of Toronto); however, Aboriginal students may not be aware 
of them or may not know how to access these services. The same lack of awareness may apply to mainstream services as well. Furthermore, services that are relevant and culturally safe for Aboriginal students are often lacking within educational institutions and, therefore, the findings from this phase highlight the need for culturally safe resources within these institutions.

In addition, these findings promote the idea that, in both clinical and educational settings, terminology should be changed to acknowledge Aboriginal worldviews and to normalize mental health concerns. In particular, terms such as 'wellness' and 'wellbeing' may be more appropriate for Aboriginal people as well as non-Aboriginal people who struggle with mental health stigma (Hinshaw, 2007). The Mental Health Commission of Canada (MHCC) published a document in 2009 with the title Toward recovery \& well-being: A framework for a mental health strategy for Canada and used the term "wellbeing" throughout, in reference to Canada's population as a whole (MHCC, 2009). Mental health, in particular, is discussed in this document as a component of wellbeing. Wellbeing is discussed as the ultimate goal and as a priority among this organization (MHCC, 2009). This provides evidence that this terminology and way of thinking is likely not unique to Aboriginal people with regard to its relevance.

KT development findings also have implications for the way in which information is provided to Aboriginal students. Aboriginal students preferred receiving information in a particular format and method and, therefore, these ideas may be applicable to mental health services and/or educational institutions. Specifically, when delivering mental health services to Aboriginal people, it might be appropriate to deliver information through storytelling, in the presence of an Elder, and incorporate traditions and ceremonies into the service, as appropriate and, when delivering information in a group format, the use of a sharing circle may be appropriate. As well, due to the emphasis on 'equal ground' and the importance of having a lack 
of hierarchy within the KT activity, it might be effective to incorporate peer support groups within the mental health system, when targeting Aboriginal people. Many Aboriginal services are currently using similar methods to deliver mental health information and treatment (e.g., Anishnawbe Health Toronto, 2011 and Soul of the Mother n.d). Regarding educational institutions, these preferences could be incorporated into services that Aboriginal students can access or into the classroom to help Aboriginal students learn. Incorporating Aboriginal content into the curricula could be a significant source of wellbeing for Aboriginal students at all educational levels. Faculty could invite Elders to deliver such content and it could be delivered using sharing circles and incorporating traditions and ceremonies.

\section{Knowledge Translation Phase}

Results from the Tool Development Phase were used to inform the Knowledge Translation Phase. The main findings from the Knowledge Translation Phase were that Aboriginal students, on average, reported an increased their knowledge of, and self-efficacy in, managing their mental health and wellbeing from pre to post activity; however the statistical significance of this finding could not be reported due to the small sample size and, therefore, these findings should be interpreted with caution. Due to the small sample size, we primarily focused on the qualitative portion of the results to inform the findings. Regarding feedback immediately after the activity implementation, participants liked the sense of community that the activity created and the welcoming environment that was created. Community and relationships are key components of Aboriginal culture (Smylie et al., 2003) and, therefore, this is in line with work discussed above that emphasizes the importance of community cohesion when implementing a KT strategy (e.g., McShane et al., 2006) and creating a culturally safe space for research participants (Baskin, 2005). Students recommended changes that should be made to the 
activity, including encouraging more active participation of students, that certain topics be discussed in more detail (e.g., grief and the mental health system), and that there be a cultural closing to end the sharing circle. This is in line with literature discussed above that emphasized the need for activity participation during KT in Aboriginal communities (e.g., Hanson \& Smylie, 2006). The fact that grief and the mental health system was noted as not being covered in enough detail is in line with Spiwak et al. (2012) who noted that grief is a significant issue among many Aboriginal communities and Jiwa et al. (2008) who noted that Aboriginal people are a very underserved population. Therefore, gaining knowledge on how the mental health system works as well as how it can be navigated could be very valuable information to cover in detail within an Aboriginal context. The recommendation of cultural closing is in line with Baskin (2005) who noted that cultural traditions should not be compromised and should be acknowledged and adhered to. It is also in line with Chandler and Lalonde $(1998 ; 2008)$ who noted that cultural connection could enhance wellbeing among Aboriginal communities. Finally, the cultural closing theme is fitting with Hanson and Smylie (2006) who recommended that culture and traditions be acknowledged when implementing KT activities within Aboriginal contexts. It is clear from the results of this phase, in addition to phase one, that Aboriginal students and staff felt strongly about having their traditions and perspectives recognized and implemented over the course of this study, and every attempt was made to respect these requests.

Upon being interviewed a month later, students were able to retain 'a good amount' of information, on average, and reported 'some' confidence in their ability to manage their mental health and wellbeing, on average. It should be noted that the statistical significance of this pre/post difference was not calculated due to the small sample size and, therefore, these findings should be interpreted with caution. An emphasis was on the qualitative findings of this phase 
with regard to interpretation. Participants specifically retained information on how to cope using one's cultural connection and community, resources one can access, and noted that some knowledge was reinforced through the activity. The fact that information on coping and resources was retained is not surprising, since these topics were identified as specific needs of Aboriginal students during the first phase of the study and are supported as knowledge needs within the current literature (e.g., Connors, 2007 noted the need for coping resources and Jiwa et al., 2008 noted the need for resources among Aboriginal communities).

In addition to the saliency of the activity topics potentially aiding in knowledge uptake and retention, participants specifically noted aspects of the activity's method that were helpful in aiding knowledge uptake and retention a month after the activity. Helpful aspects of the method included availability of food, resources provided in booklet format, the small number of people in attendance and the speakers who presented. This feedback supports the effective nature of the design of the KT activity in facilitating knowledge uptake and retention. The design of the KT phase was developed through student and staff responses from the first phase, literature on KT strategies in Aboriginal communities (e.g., Hanson \& Smylie, 2006; MacCaulay et al., 1997; McShane et al., 2006; Smylie, 2009), as well as feedback and suggestions from the Aboriginal Elder who helped the principal investigator (CJD) solidify the initial design as based on participant feedback during phase one.

Students made recommendations regarding what could be changed to increase knowledge retention a month after activity implementation, including more active participation, using both oral and visual methods of teaching, and closing the circle after the activity was complete. The recommendations for more active participation and closing the circle are in line with literature discussed above regarding recommendations for effective KT activities, which included active 
participation (Hanson \& Smylie, 2006) and Connor's (2007) emphasis on the importance of cultural connection for wellbeing among Aboriginal people. The suggestion to include both oral and visual methods of learning reflect the adaptation of Aboriginal people to alternative forms of learning over the generations, as oral forms of learning was the traditional way (Smylie et al., 2003), but the need to adapt to other forms of learning has been required through colonization.

The KT activity was designed using the knowledge to action model, which is a commonly used and mainstream model within the KT field (Graham et al., 2006). The model states that, after knowledge is created and becomes refined, it is applied (or put into action) to appropriate contexts (Graham et al., 2006). Knowledge was first created through phase one, refined through the phase one analysis as well as through feedback from the Aboriginal Elder with whom the principal investigator (CJD) worked. Once knowledge was refined, it was communicated to Aboriginal students through the KT activity and participants were given a month to further uptake this knowledge and apply it as was relevant to them (i.e., allowing them to put knowledge gained into action; Graham et al., 2006). On average, participants reported using the knowledge gained during the activity between 'some' and 'a good amount'. Specifically, they reported using cultural connection to cope and using individual coping strategies. Therefore, the only knowledge that was reported as being used was knowledge they had gained from the coping strategies portion of the activity. The fact that participants were using knowledge gained during the activity supports the effectiveness of the KT activity design as well as the successful application of the knowledge to action model (Graham et al., 2006; Wilson, Brady, \& Lesesne, 2011) to this Aboriginal context. It should be noted, however, that knowledge use at one-month follow-up may have been seen, even more so, if an Aboriginal specific model was applied to this phase of the project. Through the application of the knowledge 
to action KT model, the aim of this phase was not only to provide mental health information to Aboriginal students in a way that would enable uptake of this knowledge, but also to provide it in a way that would allow them to retain such knowledge and use it in their daily lives, which this study was able to accomplish to some degree. It should be noted that the results discussed might not be representative of the actual amount of knowledge attained by this community, since the project elicited conversations over and above the formal data collection portions of the project and, therefore, there is likely knowledge attainment and retention that simply could not be captured by this dissertation.

A common thread found in the Knowledge Translation Phase was regarding advocacy and acknowledgement of Aboriginal worldviews. In particular, during the follow-up interviews, participants emphasized the importance of being taught to advocate for themselves during the KT activity, as well as the frustration around their perspectives not being acknowledged within the mainstream system. In advocating for oneself, there is a push for such acknowledgement. This is in line with recent movements such as Idle No More, where Aboriginal community members are standing up for their political rights and expecting their voices to be heard and validated. Through Idle No More, Aboriginal people are speaking out against harmful policies that will remove Aboriginal people from even more land than has already been taken away and that can severely interrupt the environment on which we all live (Idle No More, n.d.). There is also a push for cultural restoration and revitalization through Idle No More as well as Aboriginal community members who are contributing valuable literature to this field (e.g., Battiste, 2000; Baskin, 2005; Hart, 2007; Gone, 2011; Stewart, 2011) or impacting various institutions to acknowledge and validate Aboriginal culture and perspectives (e.g., Aboriginal Education Council and Ryerson Aboriginal Student Services). 
Finally, participants in this phase expressed their thoughts regarding the overall messages that they received from the project, ongoing challenges in the area of mental health, and the future of mental health. Regarding global messages, participants noted culturally-specific messages, including how to view mental health from an Aboriginal worldview, as well as individual global messages, such as the need to address one's individual mental health concerns. Regarding ongoing challenges, participants noted that the clash of Aboriginal and Western worldviews and mental health stigma continue to exist, which creates barriers to accessing services. Participants called for the need to rekindle a sense of family and community to address mental health issues within one's community. Regarding the future of mental health, participants expressed that resources need to target Aboriginal youth, that there is a need for cultural revitalization, and that education can be used as a way forward.

There is a call for Aboriginal perspectives and worldviews to be acknowledged within the current system, including the mental health and education systems, which will challenge the status quo of mainstream psychology. This voice is getting stronger through various Indigenous movements and voices. The qualitative finding that the knowledge translation activity, as developed from results of the study's first phase, was in some ways helpful for Aboriginal students' wellbeing, further supports the necessity of incorporating Aboriginal worldviews as well as KT preferences into the mental health and educational system, as was outlined above. These findings also draw attention to the frustration among many Aboriginal people with the clash between Aboriginal and positivist Western worldviews. Aboriginal perspectives need to be recognized and fostered within the mainstreams system, especially, the mental health and education systems, in order to promote wellbeing. The implications from this phase, as well as 
the phase previously discussed, can significantly change the current system and aid Aboriginal people in achieving their educational goals.

\section{Follow-Up/Debriefing Phase}

The final phase involved key informants of the project and aimed to follow-up and debrief with them about how the project went. The main findings in the Debriefing and Followup Phase were that the project provided a sense of community, and allowed for growth and development among students, staff and the principal investigator (CJD). This is in line with the

aims and goals of community-based research, especially since it aims to benefit all community members in contrast to merely benefitting the researcher's goals, as is often the case in traditional research (Cornwall \& Jewkes, 1995). It was reported by some participants in this phase that the project benefited community members by increasing community cohesion and contributing to growth and learning among staff, students and the principal investigator (CJD). This is in line with the tenets of critical psychology and community-based research, since the goal was to benefit community members through research and is in contrast to the aims of mainstream psychology, which rarely acknowledges the impact of research on community members (Fox et al., 2009). In particular, Humphreys (2001) has described 'research' as a dirty word within an Aboriginal context due to the fact that it was historically done without regard for how such research would impact the community's wellbeing. Community-based research aims to conduct research with Aboriginal communities in order to meet the needs of community members (Cornwall \& Jewkes, 1995; Israel, 2001), which is exactly the approach that was used in the present study. All of these ideas are in line with social constructionism, since critical psychology often stems from social constructionist principles and the way in which research is now conducted in community-based settings can be understood as a result of social discourse, 
resulting in a construction of a different form of research approach that considers the social position of the populations of interest (Burr, 2003).

It was also noted by key informants that there were pros and cons of the methodology used to collect the data, but that it was important that the method aimed to build and maintain relationships between the principal investigator (CJD) and the community participants. This is in line with Baskin (2005) as well as the Tri-Council Policy Statement (Government of Canada, 2012) who both emphasized the importance of relationship building within the research process, especially when conducting research in an Aboriginal context. The relationship building emphasis throughout this project is in contrast to mainstream psychological research, where such relationships are often viewed as unethical (i.e., dual relationships; Sinclair \& Pettifor, 2001); however, Aboriginal communities have identified relationship building as a necessity within the research process (Baskin, 2005; Hanson \& Smylie, 2006).

Key informants outlined how they saw their roles in the project, which included supporting relationship building and providing spiritual/traditional support. It is recommended by the Tri-Council Policy Statement (Government of Canada, 2012) and through tenets of community-based research (e.g., Cornwall \& Jewkes, 1995) that the community be engaged in the research process. Key informants of the project contributed in various ways and the focus on relationships as well as spiritual support is in line with the Aboriginal way (Hanson \& Smylie, 2006).

Key informants noted that the project addressed some community needs including capacity building, which is in line with the aims of community-based research (Cornwall \& Jewkes, 1995). As well, key informants expressed that the project normalized mental health and provided resources, both of which have been identified as needs among Aboriginal communities 
within the current literature. Specifically, Issacs et al. (2010) has noted the need to address mental health stigma among Aboriginal communities and Jiwa et al. (2008) has cited the need for culturally safe resources for Aboriginal communities. Community based research aims to, not only address the research questions of interest, but also to address needs within the community that have been identified by community members (Cornwall \& Jewkes; Wallerstein \& Duran, 2003). This is again in line with the tenets of critical psychology and community based research and, therefore, is in contrast to mainstream psychological research, which does not take the community into account and is instead focused on the aims and needs of the research team (Cornwall \& Jewkes, 1995; Fox et al., 2009). This community-based approach is also in line with expectations of Aboriginal community members. In particular, Jacklin \& Kinoshameg (2008) writes about conducting research with Aboriginal communities 'only if it's going to mean something' for community members and emphasizes community control of research and the importance of empowerment within the research process.

Finally, key informants discussed the overarching, global messages from the project related to community specific messages as well as messages related to cultural revitalization and self-determination. Various Aboriginal authors have called for the reclamation and revitalization of Aboriginal voices, culture, and traditions (e.g., Battiste, 2000; Hart, 2007). Through cultural revitalization and connection, wellbeing has been shown to increase among Aboriginal community members (Chandler \& Lalonde, 2008). As well, Asch (2002) emphasized the importance of self-determination among Aboriginal people. Specifically, Asch (2002) discussed Aboriginal people's right to self-government and expressed Aboriginal people's ability to move toward self-sufficiency and away from reliance on governmental influences, which is in line with key informants' emphasis on this concept. 
Key informants provided insight regarding the present and future state of mental health knowledge among Aboriginal students, emphasizing that stigma continues to exist and needs to be addressed, that more resources are needed for Aboriginal people, and that institutions need to work toward Indigenizing themselves. Regarding Indigenizing institutions, it has been recommended that Indigenous knowledge be incorporated into educational curriculum across all fields of study, including psychology (Baskin, 2011; Battiste, 2000). This is just one example of the ways in which institutions can begin to acknowledge and recognize Aboriginal perspectives. It has also been stated that culture equals healing and that this idea should be incorporated in to healing models within mainstream psychological services (Aboriginal Healing Foundation, 2008; Gone, 2011; McCormick, 2000). These recommendations are in line with the tenets of critical psychology and community-based research, as they challenge the status quo regarding how mental health and these services have been traditionally conceptualized (Fox et al., 2009; Cornwall \& Jewkes, 1995). Key informants in this study called for the recognition of Aboriginal people and worldviews within the current system, as such acknowledgement is currently a rare occurrence.

Interestingly, many key informants spoke about how the project impacted them in various ways, such as motivating them to continue with their educational goals or priming them to think about the students in a different way, in order to help them even more effectively. This is in line with the aims of community-based research, as it seeks to engage and benefit as well as build capacity community members (Cornwall \& Jewkes, 1995). This feedback and discussion of such feedback is in contrast to traditional psychological research and is, therefore, in line with the tenets of critical psychology, since it emphasizes the relationship between the research process, the principal investigator (CJD) and the community members, which is not done when working 
from a positivist mainstream psychological lens (Fox et al., 2009). The feedback from key informants, regarding the ability of this project to follow the tenets of community-based research is in line with other community-based work that has been described (Fletcher, 2003; Jacklin \& Kinoshameg, 2008; Lather \& Smithies, 1997). Following the tenets of community-based research is in contrast to the way that research has traditionally been conducted with Aboriginal communities. Lather and Smithies (1997) described how the process of 'knowing' is often intrusive and exploitive (Lather \& Smithies, 1997), as is in line with Humphreys (2000) who outlines the traditional exploitive and, as a result, traumatizing nature of research in an Aboriginal context over generations. It is clear from the results of phase three that the traditional divide between the researcher and the researched is very different when using a communitybased approach, especially in an Aboriginal context, and this is supported through various community-based work from authors, including Fletcher (2003), Jacklin and Kinoshameg (2008), Lather and Smithies (1997), McShane et al. (2006), and Hartmann and Gone (2012).

Common threads among the findings from this phase were regarding the importance of establishing, maintaining and acknowledging relationships and interconnectedness between the research process, the principal investigator (CJD), and the community members, which is a very common theme within Aboriginal culture and is emphasized by Hanson and Smylie (2006), and Baksin (2005) when conducting research in an Aboriginal context. As well, themes found in this phase emphasized a push toward cultural revitalization, which is also a very common theme within Indigenous literature, including work by Baskin (2005), Battiste (2000) Hart (2007), Gone (2012), Schnarch and First Nations Centre, \& National Aboriginal Health Organization (2004), to name a few. 
There are important implications that stem from the findings of the Follow-Up and Debriefing Phase. In particular, this phase challenged mainstream thinking about how to conduct research with communities from a minority culture in a way that does not further marginalize and oppress community members, but rather contributes to their wellbeing and builds capacity within these communities. These results also provide insight regarding how projects that are similar to the present study could be implemented in various community-based organizations, particularly research with Aboriginal services within an educational institution to target mental health barriers to educational attainment experienced by Aboriginal students.

\section{Study Strengths}

A strength of this study was that a community-based approach was used throughout all three phases. In particular, the community partner (i.e., RASS), in collaboration with the principal investigator (CJD), developed the project idea and research questions. As well, community members were involved in data analysis, data interpretation as well as editing the final dissertation. In particular, the former Academic Advisor, with whom the initial dissertation idea began, read and provided feedback on the final dissertation and a research assistant (NW), who was also a participant in the second phase of the dissertation, did the same. In addition to this being appropriate and expected by a community-based research process, it is considered a strength of the project because it allowed the community to become engaged in the study and allowed everyone involved to become invested in the project, with the belief that it would address community needs. Related to the strength of using a community-based approach in this project was the design of this study to incorporate an entire phase dedicated to examining how well the project incorporated this approach. Feedback from this phase was positive, which also contributes strength to this study. This phase was also seen by the principal investigator (CJD) as 
a way to close the research circle, which is in line with Aboriginal culture and traditions (Ontario Curriculum, 2006).

Another strength of the project was the primarily qualitative method through which data were collected and analyzed. Using qualitative methods within an Aboriginal context is considered culturally safe, as it allows participants to have their voices heard, in contrast to quantitative methods that risk continuing to silence and oppress a population that has been historically traumatized through such silencing and oppression, over generations. In addition, qualitative methods allow Aboriginal participants to tell their stories, which is in line with the way in which knowledge was traditionally exchanged (Baskin, 2005) and, therefore, can create a validating research environment that incorporates Aboriginal culture and perspectives. Similarly to qualitative research methods being culturally safe, data collection procedures used in all three phases were designed to be culturally safe, which is considered to be another strength of this project. In particular, sharing circles were used to collect group data, cultural ceremonies were incorporated (e.g., smudging, opening and closing ceremonies, gifting tobacco) and Elders were included in every phase of the project as both participants and key informants of the study. As well, food was provided to participants for two of the three phases and appropriate monetary compensation as well as gifts were provided to Elders and other knowledge holders that took part in the project. Finally, the fact that social constructionism was used for phases one and three is considered to be a strength, since this lens is very much in line with the Aboriginal worldview and aimed to empower participants by recognizing and emphasizing the Aboriginal way of defining mental health and KT, as well as the Aboriginal way of conducting psychological research. 
Another strength of this study relates to the fact that data were collected during phase two using questionnaires that were specifically developed for the purposes of this study. Prior to the implementation of phase two, Aboriginal students from Ryerson University first piloted these questionnaires in order to ensure that the questionnaires were valid and that participants could understand each question. Data were coded using two different coders, both of whom identified as being Aboriginal, to work to legitimate and corroborate the findings.

\section{Study Limitations}

In spite of the many strengths of this project, there are limitations that should be acknowledged. The first limitation is the limited sample size for all three phases. Regarding the first phase, in particular, it was difficult to recruit participants due to a lack of relationship between the principal investigator (CJD) and the research participants. As relationships formed, it became easier to recruit participants; however, more relationships would have resulted in larger sample sizes within all three phases. It was unclear if saturation was reached for phase two, specifically, since only four students participated in the Knowledge Translation Phase. As a result of the small sample size in phase two, it was difficult to conduct a highly rigourous evaluation; however, in spite of the low sample size, deep qualitative data was collected during this phase and, therefore, contributed to some understanding of its effectiveness. Phase two should be replicated in the future in order to further examine the effectiveness of a knowledge translation activity within this context. As well, regarding the self-report measures provided during the Knowledge Translation Phase, it is possible that students rated their mental health, knowledge, self-efficacy, retention, and use as higher than they would have done if this were measured more objectively. Social desirability may have impacted their ratings on these questionnaires and, therefore, may have contributed to an inaccurate picture regarding any 
change in their knowledge or self-efficacy from pre to post KT activity, in particular. It is also possible that the time between the KT activity and the follow-up interview was too long. Although many participants retained information one month later and this timeline was supported by Sudsawad (2007), as well as a key informant of the project (i.e., who suggested that participants be given a full moon cycle, which is a month's time, to solidify the information provided), there were reports from some participants that they had difficulty remembering the activity itself as well as particular portions of the activity. It is also possible that participants had difficulty remembering portions of the activity because of the method through which this information was delivered (i.e., in a way that was not conducive to knowledge retention, such as limited opportunities for active participation). The Knowledge Translation Phase could have been strengthened if it was first piloted, revised based on feedback, and then implemented again. Due to the purpose and timeline of this project, this idea was not feasible to implement, but it is recommended that the activity be implemented again, while incorporating the recommended changes provided by participants, and then evaluated once again. It should also be noted that, since it has been recommended by the Canadian Institutes of Health Research (2009) that both Aboriginal and Western ways of knowing be used, due to the value in both perspectives, a mainstream model of KT was used in the present project, called the Knowledge to Action model. Although the application of this model to this project resulted in knowledge use by participants, as was the goal of this study, use of such knowledge might have increased if a KT model was used that had been established as culturally safe for use within an Aboriginal context. The use of a mainstream KT model is, therefore, recognized as a limitation of this project.

Finally, it should be noted that this project was done with a very specific population that included Aboriginal post-secondary students at Ryerson University in Toronto, Ontario as well 
as University educated Aboriginal staff, most of whom identified as being First Nations.

Although there were participants who identified as Métis, this was rare and no one in this study identified as Inuit to the knowledge of the principal investigator (CJD). Therefore, these findings should not be generalized to those outside of this context. The assumption of homogeneity among Aboriginal people in Canada has been historically traumatizing and, therefore, it is recommended against making this assumption and applying the results of this study to all Aboriginal people in Canada.

\section{Future Research}

This dissertation is merely a starting point for much additional future work that can be conducted in the area of mental health and wellbeing as well as KT from an Aboriginal perspective. Future research should further the present study by revising the KT activity based on feedback reported by students and implementing as well as evaluating the activity again for refinement, so that it can become even more beneficial for Aboriginal students at Ryerson University. In addition, this project could be expanded to include Aboriginal students from other Universities and even to students in the primary and elementary levels of education.

Furthermore, this study could be conducted within mental health agencies, including Aboriginal patients of various ages and from various Nations. It could be beneficial to understand how Aboriginal people within various social contexts view mental health and their preferences for receiving mental health knowledge as well as resources to promote their wellbeing. Due to a large focus on cultural connection and cultural revitalization within all three phases of this project, it is recommended that future research examine how the inclusion of culture and spirituality into various institutions, such as mental health services and the educational system, may impact wellbeing among Aboriginal community members. Furthermore, it is recommended 
that future work incorporate these findings into the current mental health system (e.g., acknowledging mental health from an Aboriginal worldview within various institutions). The principle investigator (CJD) has plans to continue this work and this dissertation calls for future work to use the findings of this dissertation to inform practice and institutional decision-making. With regard to recommendations made by participants, future research should work to develop strategies aimed a normalizing mental health; target youth with regarding resources; work to revitalize Aboriginal culture in various institutions, programs and services; find ways to facilitate education among Aboriginal people; and work toward indigenizing institutions, including educational and mental health institutions.

\section{Summary and Conclusions}

The study aims were: (1) to understand mental health as defined from an Aboriginal perspective as well as to develop a $\mathrm{KT}$ activity to communicate relevant mental health knowledge to Aboriginal post-secondary students at Ryerson University, (2) to implement and evaluate a KT activity developed for Aboriginal students at Ryerson University, and (3) to follow-up and debrief with key informant community members regarding the community based research process. The present study contributes rich and detailed data that addressed all three aims presented above. The overarching goal of this project was to provide Aboriginal students with knowledge and resources to address mental health barriers that many Aboriginal students may experience while trying to achieve their educational goals (Holmes, 2006).

Regarding (1), a mental health definition and KT activity were developed as stemming from an Aboriginal perspective. Specifically, mental health was defined wholistically, as related to community and as being impacted by identity, stigma, cultural connection, intergenerational trauma, and a clash between Aboriginal and positivist Western worldviews. KT preferences 
included incorporating traditional ceremonies and practices; covering particular topics such as resources, traditional teachings and coping strategies; and incorporating active participation. Regarding (2), the knowledge translation activity increased knowledge and self-efficacy related to mental health and wellbeing and some knowledge was retained and used at one-month followup. As well, participants appreciated the sense of community that the activity created and provided feedback regarding what could be changed (e.g., more opportunities for active participation). Regarding (3), key informants emphasized that the project was well received, addressed community needs and was built on relationships, all of which was in line with the aims of this project in using a community-based approach.

Implications of these findings have the potential to span various systemic arenas, including, but not limited to the mental health and educational systems. Findings from this study can be used as a training guide to facilitate an understanding within these systems regarding Aboriginal worldviews, in general, as well as Aboriginal perspectives on mental health and wellbeing, more specifically. As well, the results of this study can inform both the mental health and educational systems about how to communicate mental health information to Aboriginal students. These results can also be used to tap into ways to incorporate Aboriginal content into the current education curriculum, which can promote wellbeing through the acknowledgement of Aboriginal people and perspectives. There is a need for the field of psychology to create spaces that will invite Aboriginal resource people and practitioners to develop learning streams that are specific to Aboriginal students. Aboriginal learners are frequently being invalidated in various institutions, especially those that are educational. This project highlights current gaps as related to mental health services within educational institutions. It is recommended that the gaps outlined in this dissertation be acknowledged and used as a way to move institutions forward 
through recognizing Aboriginal people in the system. The highlighted gaps will be used by the principal investigator (CJD) as a guide for next steps, which will involve discussing these dissertation findings with community members and collaborating on plans to move forward with these results. In particular, the language used to describe mental health and mental health services/resources might need to be reconsidered within the present education system. This language stems from mainstream perspectives and worldviews and so it can be commonly invalidating for Aboriginal student within these systems. As has been demonstrated by this project, mental health can be seen as a wholistic concept and, therefore, cannot be separated from physical, emotional and spiritual health. Therefore, the frequent invalidation of Aboriginal people and their worldviews within current society will continue to impact their wellbeing in undesirable ways, creating barriers to educational attainment, and will ultimately adversely impact the Canadian society as a whole. Finally, this project can be used to demonstrate how community-based research can be done in collaboration with Aboriginal organizations and how beneficial this can be for such community members.

As is in line with Aboriginal perspectives, everyone, across nations, are connected and related to one another and, therefore, if one community within a larger society is struggling mentally, emotionally, physically, and/or spiritually, this will have an impact on everyone in some way. Yet, when all are functioning well: physically, emotionally, mentally, and spiritually, this will promote a healthy space for all. This project calls for attention and acknowledgement of Aboriginal worldviews within the mental health and KT fields as well as acknowledgment of Indigenous research methodologies in order to promote wellbeing among Aboriginal postsecondary students to break down any barriers to educational attainment. The results of this dissertation can be used to inform various services and institutions regarding the 
acknowledgement of Aboriginal Peoples in Canada as well as their perspectives on mental health and wellbeing. An increase in acknowledgement and understanding within Canada's society can promote wellbeing for all. 
Table 1.

Knowledge translation phase participant demographics $(n=4)$

\begin{tabular}{|c|c|c|c|c|}
\hline Characteristic & Mean & $\begin{array}{c}\text { Standard } \\
\text { Deviation }\end{array}$ & Range & $\mathrm{N}(\%)$ \\
\hline Age (years) & 35.75 & 15.52 & $27-59$ & \\
\hline \multicolumn{5}{|l|}{ Gender } \\
\hline Male & & & & $1(25 \%)$ \\
\hline Female & & & & $3(75 \%)$ \\
\hline \multicolumn{5}{|l|}{ Aboriginal Identity } \\
\hline First Nations & & & & $4(100 \%)$ \\
\hline Métis & & & & 0 \\
\hline Inuit & & & & 0 \\
\hline $\begin{array}{l}\text { Identification with } \\
\text { Aboriginal heritage }\end{array}$ & 4.67 & .58 & $4-5$ & \\
\hline
\end{tabular}

Note: Identification with Aboriginal heritage was measured using a 1 ( $0 \%$ identification) to 5 (100\% identification) Likert scale. 
Table 2.

Phase two mental health and wellbeing knowledge uptake and self-efficacy pre and post-activity scores by participant $(n=4)$

\begin{tabular}{|c|c|c|c|c|}
\hline \multirow[b]{2}{*}{ Participant } & \multicolumn{2}{|c|}{ Knowledge Uptake } & \multicolumn{2}{|c|}{ Self-Efficacy } \\
\hline & Pre Activity: & Post Activity: & Pre Activity: & Post Activity: \\
\hline & Mean(SD) & Mean(SD) & Mean (SD) & Mean(SD) \\
\hline 1 & $2.38(.52)$ & $3.25(1.04)$ & $2.67(.58)$ & $4.00(1.00)$ \\
\hline 2 & 4.14(.69) & 4.13(.99) & $4.67(.58)$ & $4.33(.58)$ \\
\hline 3 & $3.88(.83)$ & $3.75(.46)$ & $4.00(.00)$ & $4.00(.00)$ \\
\hline 4 & $3.63(.92)$ & $4.13(.83)$ & $4.33(.58)$ & $4.67(.58)$ \\
\hline
\end{tabular}


Figure 1. Mental Health Model

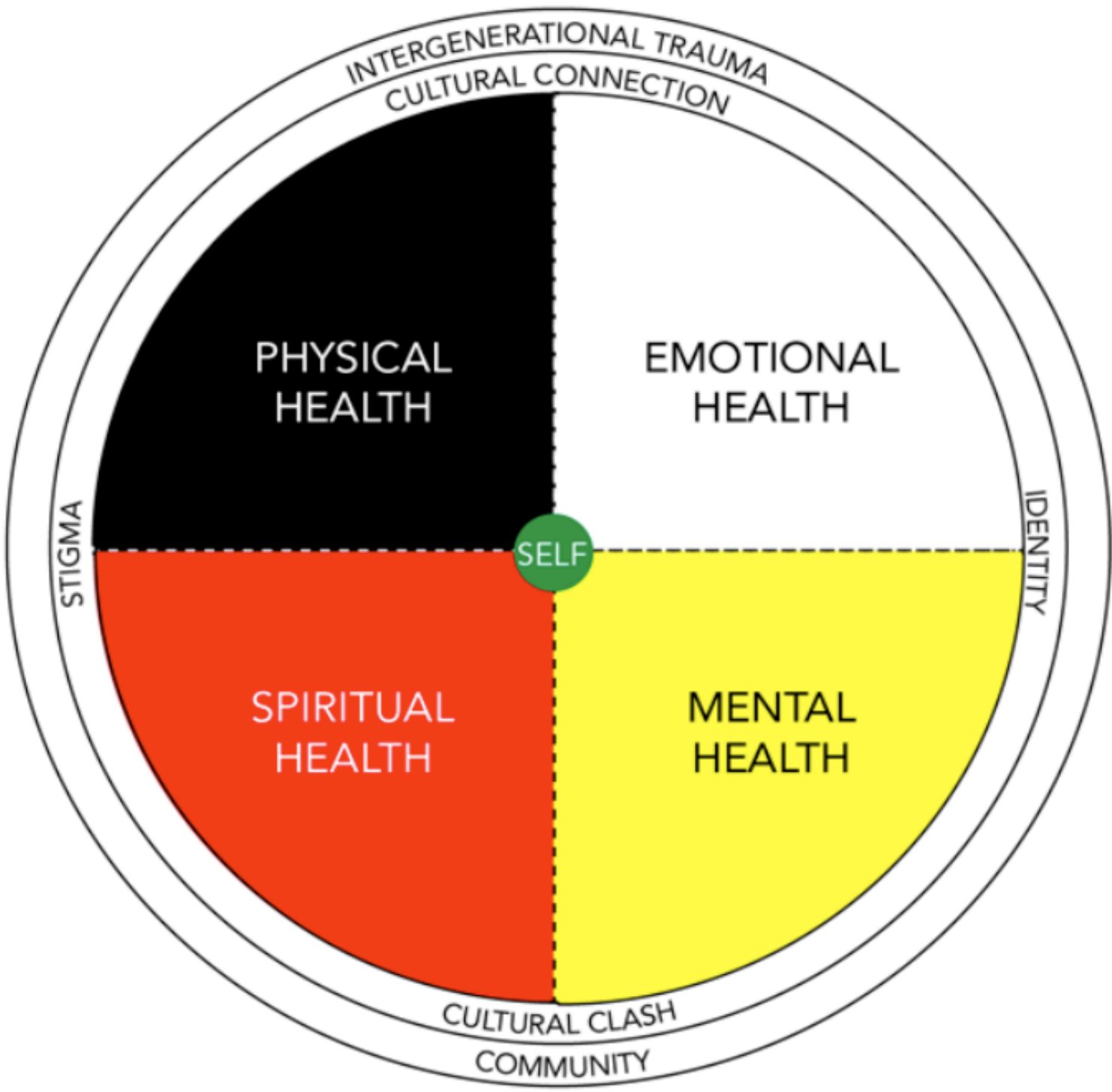

Figure 1. Mental health model from an Aboriginal worldview. As noted in the Results: Tool Development Phase section, mental health cannot be divorced from the other elements of the self (i.e., physical, emotional, and spiritual) and is impacted by stigma, cultural connection, a cultural clash, and Aboriginal identity. All of these elements are impacted by intergenerational trauma as a result of colonization and mental health is considered a community responsibility. For a more detailed discussion of this model, see the "Definition of Mental Health from an Aboriginal Perspective" section. 
Appendix A

Student Recruitment Flyer

RYERSONUNIVERSITY

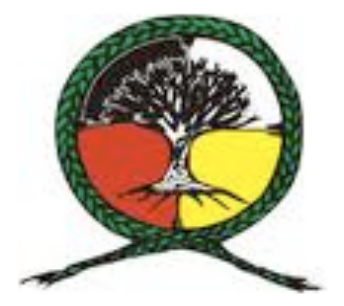

\section{Participants Needed!}

\section{Study on Aboriginal Mental Health Perspectives and Knowledge}

Do you identify as being First Nations, Inuit or Métis?

\section{Are you a student member of Ryerson Aboriginal Student Services?}

If so, and you are between the ages of 18-65, you may be eligible to participate in a research study through Ryerson University and Ryerson Aboriginal Student Services!

You will be compensated for your participation if you are eligible. For more information please contact:

Phone: (416) 979-5000 ext. 2187

Email: cdavey@psych.ryerson.ca

All queries are confidential

\begin{tabular}{|c|c|c|c|c|c|c|c|}
\hline 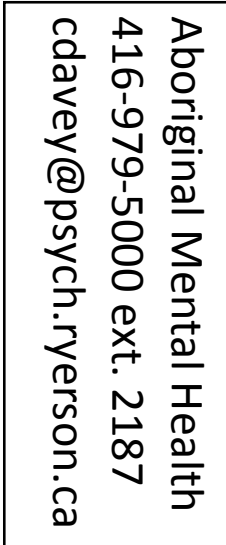 & 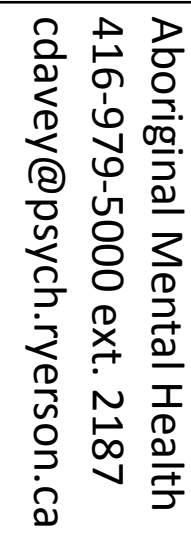 & 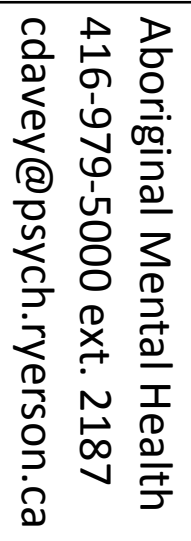 & 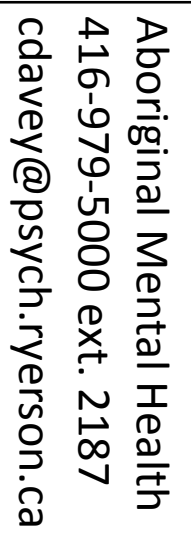 & 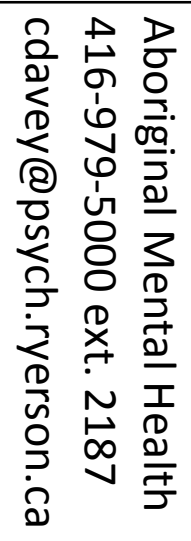 & 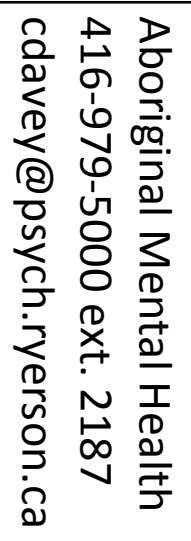 & 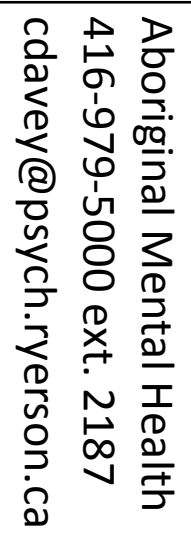 & 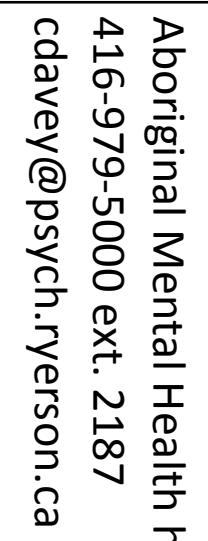 \\
\hline
\end{tabular}


Appendix $B$

Staff Recruitment Flyer

RYERSONUNIVERSITY

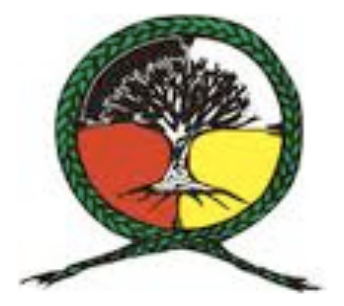

\section{Participants Needed!}

\section{Study on Aboriginal Mental Health Perspectives and Knowledge}

Do you identify as being First Nations, Inuit or Métis?

Are you a member of Ryerson Aboriginal Student Services?

\section{Have you worked with Aboriginal students?}

If so, and you are between the ages of 18-65, you may be eligible to participate in a research study through Ryerson University and Ryerson Aboriginal Student Services!

You will be compensated for your participation if you are eligible. For more information please contact:

Phone: (416) 979-5000 ext. 2187

Email: cdavey@psych.ryerson.ca

All queries are confidential

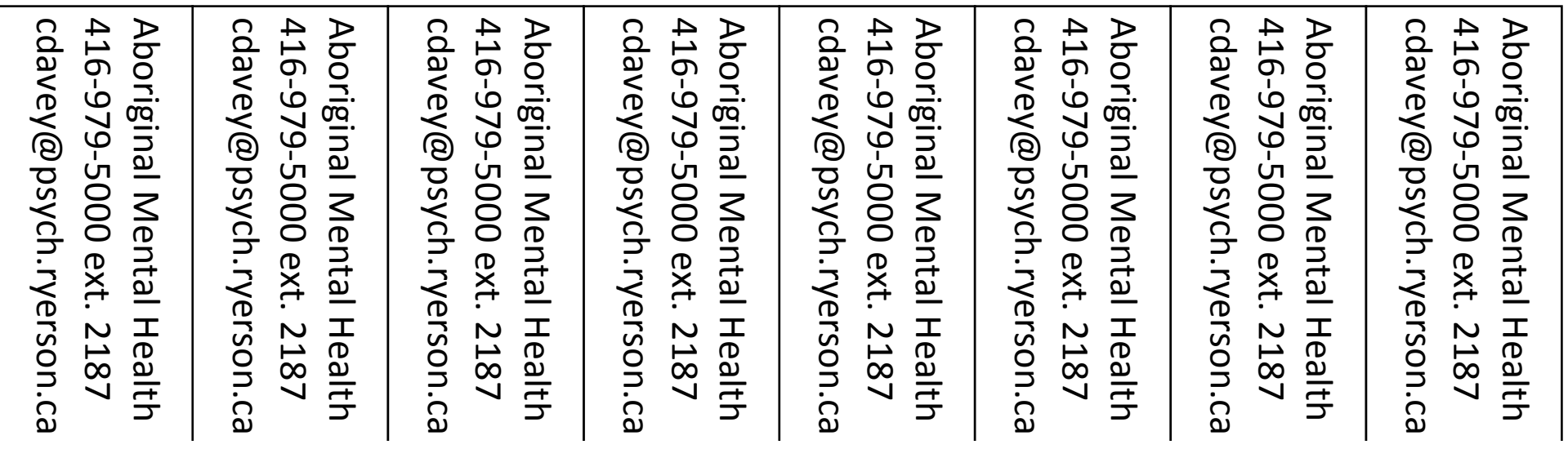




\section{Student Recruitment Script: Tool Development Phase}

[Inclusion criteria reminder:

They identify as First Nations, Inuit or Métis

They are between the ages of 18 to 65

They are a student member of Ryerson Aboriginal Student Services]

Script:

The study is called 'Aboriginal mental health perspectives and knowledge'. We are trying to understand Aboriginal perspectives on mental health, gaps in students' mental health knowledge and how to best provide Aboriginal students with mental health information to help them manage their mental health concerns. The study is being done through Ryerson University and in partnership with Ryerson Aboriginal Student Services.

The researchers are recruiting First Nations, Inuit and Métis students who are members of Ryerson Aboriginal Student Services. You would participate in a focus group with 10 to 12 other students, during which you would be asked about your thoughts about mental health and preferences for receiving mental health information.

The focus group will take about an hour and a half and you will be compensated $\$ 20$ for your participation. Participation in this study is voluntary, which means that your relationship with me, Ryerson University, and the Ryerson Aboriginal Student Services will not be affected if you refuse." 


\section{Appendix D}

\section{Staff Recruitment Guiding Script: Tool Development Phase}

[Inclusion criteria reminder:

They identify as First Nations, Inuit or Métis

They are between the ages of 18 to 65

They are a past or present member of Ryerson Aboriginal Student Services

They have worked with Aboriginal students either presently or in the past]

Script:

"The study is called 'Aboriginal mental health perspectives and knowledge'. We are trying to understand Aboriginal perspectives on mental health, gaps in students' mental health knowledge and how to best provide Aboriginal students with mental health information to help them manage their mental health concerns. The study is being done through Ryerson University and in partnership with Ryerson Aboriginal Student Services.

The researchers are recruiting First Nations, Inuit and Métis community members who are members of Ryerson Aboriginal Student Services and who have worked with Aboriginal students. You would participate in a focus group with 10 to 12 other community members, during which you would be asked about your thoughts about mental health and ways that you have effectively communicated knowledge to Aboriginal students.

The focus group will take about an hour and a half and you will be compensated $\$ 20$ for your participation. Participation in this study is voluntary, which means that your relationship with me, Ryerson University, and the Ryerson Aboriginal Student Services will not be affected if you refuse." 
Appendix E

\section{Student Recruitment Script: Knowledge Translation Phase}

[Inclusion criteria reminder:

They identify as First Nations, Inuit or Métis

They are between the ages of 18 to 65

They are a student member of Ryerson Aboriginal Student Services

If you are recruiting for the Final KT strategy and they have participated in the pilot KT strategy, they are not eligible to participate in the Final KT strategy]

Script:

"This study is called 'Aboriginal mental health perspectives and knowledge'. We are trying to understand Aboriginal perspectives on mental health, gaps in students' mental health knowledge and how to best provide Aboriginal students with mental health information to help them manage their mental health concerns. The study is being done through Ryerson University and in partnership with Ryerson Aboriginal Student Services.

The researchers are recruiting First Nations, Inuit and Métis students who are members of Ryerson Aboriginal Student Services. You would participate in a Wellbeing Activity to teach students how to manage their wellbeing and increase confidence in these skills. You would also be asked about your thoughts about mental health and your own confidence in your ability to manage your mental health concerns prior to as well as upon completion of the activity. The knowledge translation activity will be a day event and you will be compensated $\$ 25$ for your participation.

You will also be asked to participate in an individual interview a month after the knowledge Wellbeing Activity, which will last about a 30 minutes. During this interview, you will be asked questions about how the Wellbeing Activity worked or did not work for you as well as how you have or have not incorporated wellbeing strategies into your daily life. You will be compensated $\$ 10$ for your participation in this interview for a total of $\$ 35$ for participating in both the knowledge translation activity and the individual interview a month later.

Participation in this study is voluntary, which means that your relationship with me, Ryerson University, and the Ryerson Aboriginal Student Services will not be affected if you refuse." 


\section{Key Informant Email Recruitment Script}

\section{Appendix $F$}

Hello [name of key informant],

I have completed all the data collection for my dissertation and want to thank you for all of your support throughout this process. To finalize some of the concepts that I have found and to close the circle of data collection, I was wondering if you might be willing to meet with me for about 30 to 45 minutes so that I can ask you some follow-up questions.

We can meet in your office or somewhere at RASS if this is convenient for you. I can also pay you $\$ 10$ for your participation. I would like to use your responses to my questions as data in my dissertation (de-identified, of course) and so I hope to audio record you, but we can talk more about that when we meet.

Please let me know if you would be willing to meet with me and we can set up a time and date. Thank you again for the time and support that you have already given me throughout this project and I hope that you will be able to meet with me again soon.

Thanks,

Caitlin 


\section{RASS Tool Development Consent Form}

\section{Appendix $G$}

\section{RYERSON UNIVERSITY}

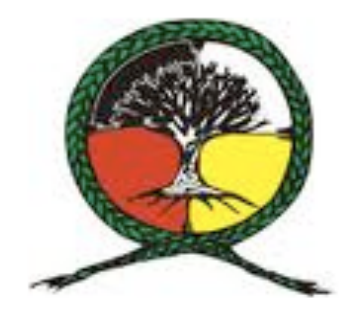

\section{Consent Agreement Tool Development Phase}

You are being asked to participate in a research study. Before you give your consent to be a volunteer, it is important that you read the following information and ask as many questions as necessary to be sure you understand what you will be asked to do.

\section{Investigators:}

Caitlin Davey, M.A.

Ph.D. Student

Department of Psychology

Ryerson University

(supervised by Kelly McShane)

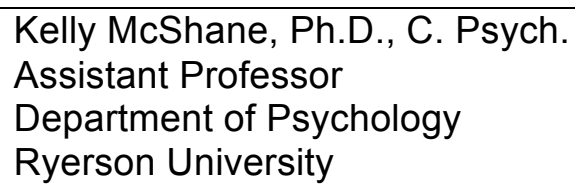

\section{Purpose of the Study:}

The purpose of this project is to understand Aboriginal perspectives of mental health, gaps in mental health knowledge among Aboriginal students, and the best ways to give Aboriginal students mental health knowledge. The results of the study aims to increase the understanding of mental health concerns among Aboriginal students and increase mental health knowledge as well as internal resources for Aboriginal students.

Description of the Study: As part of this study, you will be asked to participate in a focus group to help us understand what mental health is from an Aboriginal worldview as well as the best ways to provide mental health knowledge to Aboriginal students. This focus group will take about an hour and a half and will take place at Ryerson Aboriginal Student Services (RASS) at Ryerson University.

None of the procedures used in this study are experimental in nature. The only experimental aspect of this study is the gathering of information for the purpose of analysis.

Risks or Discomforts: It is possible that during this study you will become uncomfortable. If you begin to feel uncomfortable, you may discontinue participating.

Benefits of the Study: You may receive some benefits from being in this study. The results of this study may help you or other Aboriginal community members at Ryerson University better understand mental health. We cannot guarantee, however, that you will receive any benefits from participating in this study. 
Confidentiality: Confidentiality will be respected and no information that discloses your identity will be released or published without your consent, unless required by law. Direct quotations will be used in the publication of the data collected; however, all identifying information will be removed from the quotations. If you do not want your quotations to be used in any publications, please let the researchers know and this will be documented and respected.

I agree to have my direct quotations used

I do not agree to have my direct quotations used, but agree that what I say can be paraphrased

I do not agree to have my direct quotations used or paraphrased

The focus group will be audio taped. The digital audio files will be kept on a secure server at Ryerson University and will be password protected. No identifying information will be tied to the files. Audio files will be destroyed after we have confirmed all vital information (2-3 years). Only study staff will have access to these data. After 10 years, all information will be destroyed.

\section{Incentive to Participate}

You will be paid $\$ 20$ for your participation.

Voluntary Nature of Participation: Participation in this study is voluntary. Your choice of whether or not to participate will not influence your future relations with Ryerson University or RASS. If you decide to participate, you are free to withdraw your consent and to stop your participation at any time without penalty or loss of benefits to which you are allowed. You are also allowed to withdraw your data at a later time if you so choose.

At any particular point in the study, you may refuse to answer any particular question or stop participation altogether.

Questions about the Study: If you have any questions about the research now, please ask. If you have questions later about the research, you may contact.

Caitlin Davey, M.A.

Ph.D. Student

Department of Psychology

Ryerson University

350 Victoria Street

Toronto Ontario Canada M5B 2K3

Phone: 416-979-5000, ext 2188 (after pressing 1)

Email: cdavey@psych.ryerson.ca 
If you have questions regarding your rights as a human subject and participant in this study, you may contact the Ryerson University Research Ethics Board for information.

Dr. Nancy Walton

Research Ethics Board

c/o Office of the Vice President, Research and Innovation

Ryerson University

350 Victoria Street

Toronto, ON M5B 2K3

Phone: 416-979-5042

\section{Agreement:}

Your signature below indicates that you have read the information in this agreement and have had a chance to ask any questions you have about the study. Your signature also indicates that you agree to be in the study and have been told that you can change your mind and withdraw your consent to participate at any time. You have been given a copy of this agreement. You have been told that by signing this consent agreement you are not giving up any of your legal rights.

Name of Participant (please print)

Signature of Participant Date

Signature of Investigator

\section{Date}

Your signature below indicates that you have read and understand that you will be audiorecorded for only the purposes of this study. Your signature indicates that you agree to be audio-recorded and have been told that you can change your mind and withdraw this consent to be audio-recorded at any time.

Name of Participant (please print)

Signature of Participant

Signature of Investigator
Date

Date 


\section{Appendix $H$}

\section{Tool Development: Student Focus Group Questions}

The purpose of this group is threefold: (1) to understand mental health from an Aboriginal worldview, (2) to understand gaps to be filled regarding students' mental health knowledge, and (3) to understand the best way to provide mental health knowledge to Aboriginal students so they can better manage mental health concerns

1. What does mental health mean to you?

Probes:

-When you hear the words "mental health", what thoughts, feelings, images, or stories come to mind?

-When Aboriginal people talk about mental health, what do they say? How do they describe it? -How do you think Aboriginal views on mental health differ from non-Aboriginal views?

2. If you were experiencing a mental health concern (e.g., feeling down, anxious, etc), what would be some things you might do to make yourself feel better?

\section{Probes:}

-Who would you turn to?

-What strategies could you use to help yourself feel better?

-Do you know anyone who had a mental health concern? What kinds of things did they do to help themselves feel better?

-What are some self-care activities that might help someone feel good about themselves?

3. If you needed to get help for a mental health concern where would you go?

\section{Probes:}

-Do you know anyone who has had a mental health concern? If so, where did they go to get help? What resources did they access?

-Do you know of any mental health resources that may be helpful?

4. Is there anything about mental health that you would like to know more about?

\section{Probes:}

- Is there anything about mental health that you feel like you don't understand or that confuses you?

5. If you needed to get information about mental health, how would you get it?

\section{Probes:}

-Would you go to your family doctor? A counselor? Would you read online?

-How do you think other people would get mental health information if they wanted it?

6. If you wanted to share mental health information with Aboriginal students, how would you do it?

\section{Probes:}

-What would you do? Who should do it?

-How do you best learn? 
-What have been the best ways for you to learn about other topics in the past? What was it about those methods of learning that helped you apply that information to your life? 


\section{Appendix I}

\section{Tool Development: Staff Focus Group Questions}

The purpose of this group is fourfold: (1) to understand mental health from an Aboriginal worldview, (2) to understand gaps to be filled regarding students' mental health knowledge (3) to gain the appropriate knowledge to fill the gaps in students' mental health knowledge, and (4) to understand the best way to provide mental health knowledge to Aboriginal students so they can better manage mental health concerns

1. What does mental health mean to you?

\section{Probes:}

-When you hear the words "mental health", what thoughts, feelings, images, or stories come to mind?

-When Aboriginal people talk about mental health, what do they say? How do they describe it?

2. Is there anything about mental health that you think is commonly misunderstood or simply not known among Aboriginal students?

Probes:

-Is there anything that you have learned about mental health that most youth do not know, but it would be helpful for them to know?

3. If you were working with a student who was experiencing mental health concerns, what would you recommend for them?

Probes:

-Would you refer them to their family doctor?

-What sorts of mental health resources would you recommend?

-What sorts of internal coping strategies would you recommend?

4. You have all worked with Aboriginal students, in your experience, what have been the most effective strategies that you have used to give students information?

Probes:

-How have you found Aboriginal students to learn best?

5. Do you have any ideas about the best ways to provide Aboriginal students with mental health information so that they use it in their daily lives (e.g., a group activity, electronically, etc.)?

Probes:

-How should it be done and who should do it?

-Thinking about when you were a student or when you were a young adult (or now if you are still a young adult), how would you have wanted (or want) to receive mental health information? 
KT Phase Questionnaire Pilot

Appendix $J$

\section{Wellbeing Knowledge Uptake Questionnaire (provided pre and post activity)}

This questionnaire will be used before and after the wellbeing activity in order to determine whether knowledge about wellbeing has changed.

1. Are there any questions that are unclear to you? If so, which ones and why?

2. Are there any questions that you would change? If so, what would you change about them?

3. Are there any questions that you would add? If so, why?

4. Are there any questions you would remove? If so, why?

5. Do you think that this method of collecting information is appropriate? If not, please explain.

\section{Post Knowledge Translation Strategy Evaluation Questions}

This questionnaire will be used to evaluate the delivery of the activity to see if anything about the activity should be changed if implemented again in the future.

1. Are there any questions that were unclear to you? If so, which ones and why?

2. Are there any questions that you would change? If so, what would you change about them?

3. Are there any questions that you would add? If so, why?

4. Are there any questions you would remove? If so, why?

5. Do you think that this method of collecting information is appropriate? If not, please explain. 


\section{Knowledge to Action Interview Guide}

This questionnaire will be used one month later to ask students how much they remember from the activity and whether they are using anything they learned from the activity.

1. Are there any questions that were unclear to you? If so, which ones and why?

2. Are there any questions that you would change? If so, what would you change about them?

3. Are there any questions that you would add? If so, why?

4. Are there any questions you would remove? If so, why?

5. Do you think that this method of collecting information is appropriate? If not, please explain. 


\section{RASS Knowledge Translation Consent Form}

Appendix $K$

\section{RYERSON UNIVERSITY}

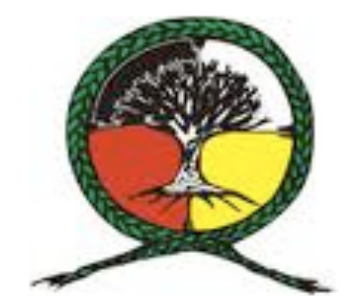

\section{Consent Agreement \\ Knowledge Translation Strategy}

You are being asked to participate in a research study. Before you give your consent to be a volunteer, it is important that you read the following information and ask as many questions as necessary to be sure you understand what you will be asked to do.

\section{Investigators:}

Caitlin Davey, M.A.

Ph.D. Student

Department of Psychology

Ryerson University

(supervised by Kelly McShane)
Kelly McShane, Ph.D., C. Psych.

Assistant Professor

Department of Psychology

Ryerson University

350 Victoria Street

Toronto, ON, M5B 2K3

416-979-5000 ext. 2051

kmcshane@psych.ryerson.ca

\section{Community Partner:}

The investigators have partnered with Ryerson Aboriginal Student Services (RASS) to complete this study. This means that RASS has identified the aims of the present study as a need for their organization and will collaborate with the research team regarding all aspects of the study (i.e., from research design to the interpretation of analysis). Ruth Koleszar-Green, BSW, MSW, RSW, is the Aboriginal Academic Support Advisor at RASS and is the RASS representative with which the investigators will be working to complete the present study. RASS staff members, including Ruth Koleszar-Green, will not have access to confidential information expressed by participants in the present study.

\section{Purpose of the Study:}

The purpose of this project is to understand Aboriginal perspectives of mental health, gaps in mental health knowledge among Aboriginal students, and the best ways to give Aboriginal students mental health knowledge. The results of the study aims to increase the understanding of mental health concerns among Aboriginal students and increase Aboriginal students' mental health knowledge and resources for coping with mental health concerns. 
Description of the Study: This study is being completed for a psychology graduate student thesis project. As part of this study, you will be asked to participate in a wellbeing activity that has been designed to provide information to Aboriginal students in a way that is beneficial to them. Before and after the activity, you will be asked questions about your knowledge related to wellbeing to assess whether your knowledge changes over the course of the activity. You will also be asked questions about your own confidence in managing your wellbeing to assess whether this confidence increases as a result of the activity. Finally, you will be asked to provide feedback on the activity. This activity will be approximately four hours in length (i.e., will span the day) and will take place at Ryerson Aboriginal Student Services (RASS) or another location at Ryerson University.

You will also be asked to return a month later for an individual interview. For the interview, you will be asked questions about how you have or plan to use the information gained in the knowledge translating activity as well as what you remember from the activity. None of the procedures used in this study are experimental in nature. This interview will take about an hour and will take place at Ryerson Aboriginal Student Services (RASS) at Ryerson University.

The only experimental aspect of this study is the gathering of information for the purpose of analysis.

Risks or Discomforts: It is possible that during this study you will become uncomfortable. During the wellbeing activity or interview, you may feel uncomfortable with topics that other participants may discuss or feel self conscious about your knowledge. If you begin to feel uncomfortable, you may discontinue participating. You may also discontinue participating for any other reason. In addition, questions addressing participants personal experiences with mental health will be not be asked.

Benefits of the Study: You may receive some benefits from being in this study. The results of this study may help you better understand mental health and increase your wellbeing. We cannot guarantee, however, that you will receive any benefits from participating in this study.

Confidentiality: Confidentiality will be respected and no information that discloses your identity will be released or published without your consent, unless required by law. The research team will maintain confidentiality, but we cannot promise this on behalf of the other participants (in the wellbeing activity), although it will be requested. Direct quotations will be used in the publication of the data collected; however, all identifying information will be removed from the quotations.

If you do not want your quotations to be used in any publications, please let the researchers know and this will be documented and respected.

I agree to have my direct quotations used I do not agree to have my direct quotations used, but agree that what I say can be paraphrased I do not agree to have my direct quotations used or paraphrased 
The results of the study will be made available to all participants before publication, so they have a chance to make changes to anything (including quotes) that they are uncomfortable with.

The activity and interview will be audio taped. The digital audio files will be kept on a secure server at Ryerson University and will be password protected. No identifying information will be tied to the files or transcripts. Audio files will be destroyed after we have confirmed all vital information (2-3 years). After this time, data (transcripts) will be transferred to RASS where they will be stored (without any identifying information tied to transcripts). At this point, only study staff will have access to these data (i.e., the principal investigators and RASS). After 10 years, all information will be destroyed.

\section{Incentive to Participate}

You will be paid $\$ 35$ total for your participation in both the activity ( $\$ 25)$ and the interview (\$10).

Voluntary Nature of Participation: Participation in this study is voluntary. Your choice of whether or not to participate will not influence your future relations with Ryerson University or RASS. If you decide to participate, you are free to withdraw your consent and to stop your participation at any time without penalty or loss of benefits to which you are allowed. You are also allowed to withdraw your data at a later time if you so choose.

At any particular point in the study, you may refuse to answer any particular question or stop participation altogether.

Questions about the Study: If you have any questions about the research now, please ask. If you have questions later about the research, you may contact.

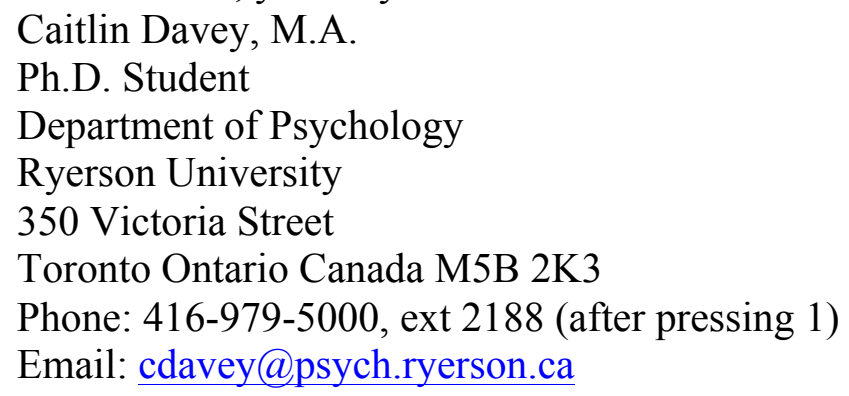

If you have questions regarding your rights as a human subject and participant in this study, you may contact the Ryerson University Research Ethics Board for information.

Dr. Lynn Lavallee

Research Ethics Board

c/o Office of the Vice President, Research and Innovation

Ryerson University

350 Victoria Street

Toronto, ON M5B 2K3

Phone: 4169795000 ext. 4791

Email: lavallee@ryerson.ca

\section{Agreement:}


Your signature below indicates that you have read the information in this agreement and have had a chance to ask any questions you have about the study. Your signature also indicates that you agree to be in the study and have been told that you can change your mind and withdraw your consent to participate at any time. You have been given a copy of this agreement.

You have been told that by signing this consent agreement you are not giving up any of your legal rights.

Name of Participant (please print)

Signature of Participant

Date

Signature of Investigator

Date

Your signature below indicates that you have read and understand that you will be audiorecorded for only the purposes of this study. Your signature indicates that you agree to be audio-recorded and have been told that you can change your mind and withdraw this consent to be audio-recorded at any time.

Name of Participant (please print)

Signature of Participant

Date

Signature of Investigator

Date 


\section{Appendix $L$}

Wellbeing Knowledge Uptake Questionnaire (pre activity)

\section{Name Initials:}

Age:

Do you identify as being, or think you are Aboriginal (yes/no):

Please specify your Aboriginal ancestry if you know it (e.g., Métis, Inuit, Ojibway, Mohawk, etc.):

Please rate on a scale from 1 to 5 how much you identify with your Aboriginal identity (1=0\% and 5 = 100\%; circle your response): $1 \quad 2 \quad 3 \quad 4 \quad 5$

1. Please rate on a scale from 1 to 5 how much knowledge you think you have about mental wellbeing (circle your response):

\begin{tabular}{|c|c|c|c|c|}
\hline None at all & A little bit & Some & $\begin{array}{l}\text { A good } \\
\text { amount }\end{array}$ & A lot \\
\hline 1 & 2 & 3 & 4 & 5 \\
\hline
\end{tabular}

2. Please rate on a scale from 1 to 5 how much knowledge you think you have about anxiety/stress (circle your response):

\begin{tabular}{|c|c|c|c|c|}
\hline None at all & A little bit & Some & $\begin{array}{l}\text { A good } \\
\text { amount }\end{array}$ & A lot \\
\hline 1 & 2 & 3 & 4 & 5 \\
\hline
\end{tabular}

3. Please rate on a scale from 1 to 5 how much knowledge you think you have about depression (circle your response):

\begin{tabular}{|c|c|c|c|c|}
\hline None at all & A little bit & Some & $\begin{array}{l}\text { A good } \\
\text { amount }\end{array}$ & A lot \\
\hline 1 & 2 & 3 & 4 & 5 \\
\hline
\end{tabular}

4. Please rate on a scale from 1 to 5 how much knowledge you think you have about addiction (circle your response):

\begin{tabular}{|c|c|c|c|c|}
\hline None at all & A little bit & Some & $\begin{array}{l}\text { A good } \\
\text { amount }\end{array}$ & A lot \\
\hline 1 & 2 & 3 & 4 & 5 \\
\hline
\end{tabular}


5. Please rate on a scale from 1 to 5 how much knowledge you think you have about suicide within Aboriginal communities (circle your response):

\begin{tabular}{|c|c|c|c|c|}
\hline None at all & A little bit & Some & $\begin{array}{l}\text { A good } \\
\text { amount }\end{array}$ & A lot \\
\hline 1 & 2 & 3 & 4 & 5 \\
\hline
\end{tabular}

6. Please rate on a scale from 1 to 5 how much knowledge you think you have about eating disorders (circle your response):

\begin{tabular}{|c|c|c|c|c|}
\hline None at all & A little bit & Some & $\begin{array}{l}\text { A good } \\
\text { amount }\end{array}$ & A lot \\
\hline 1 & 2 & 3 & 4 & 5 \\
\hline
\end{tabular}

7. Please rate on a scale from 1 to 5 how much knowledge you think you have about schizophrenia:

\begin{tabular}{|c|c|c|c|c|}
\hline None at all & A little bit & Some & $\begin{array}{l}\text { A good } \\
\text { amount }\end{array}$ & A lot \\
\hline 1 & 2 & 3 & 4 & 5 \\
\hline
\end{tabular}

8. Please rate on a scale from 1 to 5 how much knowledge you think you have about different coping strategies to manage your wellbeing (circle your response):

\begin{tabular}{|c|c|c|c|c|}
\hline None at all & A little bit & Some & $\begin{array}{l}\text { A good } \\
\text { amount }\end{array}$ & A lot \\
\hline 1 & 2 & 3 & 4 & 5 \\
\hline
\end{tabular}

9. Please rate on a scale from 1 to 5 how much confidence you have in your ability to find support/services for a mental health concern (circle your response):

\begin{tabular}{|c|c|c|c|c|}
\hline None at all & \multicolumn{2}{c}{ A little bit } & Some & \multicolumn{1}{c|}{$\begin{array}{c}\text { A good } \\
\text { amount }\end{array}$} \\
\hline 1 & 2 & 3 & 4 & 5 \\
\hline
\end{tabular}

10. Please rate on a scale from 1 to 5 how much confidence you have in your ability to find support/services for a mental health concern (circle your response):

\begin{tabular}{|c|c|c|c|c|}
\hline None at all & \multicolumn{2}{|c}{ A little bit } & Some & \multicolumn{1}{c|}{$\begin{array}{c}\text { A good } \\
\text { amount }\end{array}$} \\
\hline 1 & 2 & 3 & 4 & 5 \\
\hline
\end{tabular}

11. Please rate on a scale from 1 to 5 how much confidence you have in your ability to navigate the mental health system (e.g., ask questions and get what you need; circle your response): 


\begin{tabular}{|c|c|c|c|c|}
\hline None at all & A little bit & Some & $\begin{array}{l}\text { A good } \\
\text { amount }\end{array}$ & A lot \\
\hline 1 & 2 & 3 & 4 & 5 \\
\hline
\end{tabular}

12. Please rate on a scale from 1 to 5 how much confidence you have in your ability to manage your own mental health and wellbeing (circle your response):

\begin{tabular}{|c|c|c|c|c|}
\hline None at all & A little bit & Some & $\begin{array}{l}\text { A good } \\
\text { amount }\end{array}$ & A lot \\
\hline 1 & 2 & 3 & 4 & 5 \\
\hline
\end{tabular}

\section{Wellbeing Knowledge Uptake Questionnaire (provided post activity)}

\section{Name Initials:}

1. Please rate on a scale from 1 to 5 how much knowledge you think you have about mental wellbeing (circle your response):

\begin{tabular}{|c|c|c|c|c|}
\hline None at all & A little bit & Some & $\begin{array}{l}\text { A good } \\
\text { amount }\end{array}$ & A lot \\
\hline 1 & 2 & 3 & 4 & 5 \\
\hline
\end{tabular}

2. Please rate on a scale from 1 to 5 how much knowledge you think you have about anxiety/stress (circle your response):

\begin{tabular}{|c|c|c|c|c|}
\hline None at all & A little bit & Some & $\begin{array}{l}\text { A good } \\
\text { amount }\end{array}$ & A lot \\
\hline 1 & 2 & 3 & 4 & 5 \\
\hline
\end{tabular}

3. Please rate on a scale from 1 to 5 how much knowledge you think you have about depression (circle your response):

\begin{tabular}{|c|c|c|c|c|}
\hline None at all & A little bit & Some & $\begin{array}{l}\text { A good } \\
\text { amount }\end{array}$ & A lot \\
\hline 1 & 2 & 3 & 4 & 5 \\
\hline
\end{tabular}

4. Please rate on a scale from 1 to 5 how much knowledge you think you have about addiction (circle your response):

\begin{tabular}{|c|c|c|c|c|}
\hline None at all & \multicolumn{2}{c}{ A little bit } & Some & \multicolumn{1}{c|}{$\begin{array}{c}\text { A good } \\
\text { amount }\end{array}$} \\
\hline 1 & 2 & 3 & 4 & 5 \\
\hline
\end{tabular}

5. Please rate on a scale from 1 to 5 how much knowledge you think you have about suicide within Aboriginal communities (circle your response): 


\begin{tabular}{|c|c|c|c|c|}
\hline None at all & A little bit & Some & $\begin{array}{l}\text { A good } \\
\text { amount }\end{array}$ & A lot \\
\hline 1 & 2 & 3 & 4 & 5 \\
\hline
\end{tabular}

6. Please rate on a scale from 1 to 5 how much knowledge you think you have about eating disorders (circle your response):

\begin{tabular}{|c|c|c|c|c|}
\hline None at all & A little bit & Some & $\begin{array}{l}\text { A good } \\
\text { amount }\end{array}$ & A lot \\
\hline 1 & 2 & 3 & 4 & 5 \\
\hline
\end{tabular}

7. Please rate on a scale from 1 to 5 how much knowledge you think you have about schizophrenia:

\begin{tabular}{|c|c|c|c|c|}
\hline None at all & A little bit & Some & $\begin{array}{l}\text { A good } \\
\text { amount }\end{array}$ & A lot \\
\hline 1 & 2 & 3 & 4 & 5 \\
\hline
\end{tabular}

8. Please rate on a scale from 1 to 5 how much knowledge you think you have about different coping strategies to manage your wellbeing (circle your response):

\begin{tabular}{|c|c|c|c|c|}
\hline None at all & A little bit & Some & $\begin{array}{l}\text { A good } \\
\text { amount }\end{array}$ & A lot \\
\hline 1 & 2 & 3 & 4 & 5 \\
\hline
\end{tabular}

9. Please rate on a scale from 1 to 5 how much confidence you have in your ability to find support/services for a mental health concern (circle your response):

\begin{tabular}{|c|c|c|c|c|}
\hline None at all & A little bit & Some & $\begin{array}{l}\text { A good } \\
\text { amount }\end{array}$ & A lot \\
\hline 1 & 2 & 3 & 4 & 5 \\
\hline
\end{tabular}

10. Please rate on a scale from 1 to 5 how much confidence you have in your ability to find support/services for a mental health concern (circle your response):

\begin{tabular}{|c|c|c|c|c|}
\hline None at all & A little bit & Some & $\begin{array}{l}\text { A good } \\
\text { amount }\end{array}$ & A lot \\
\hline 1 & 2 & 3 & 4 & 5 \\
\hline
\end{tabular}

11. Please rate on a scale from 1 to 5 how much confidence you have in your ability to navigate the mental health system (e.g., ask questions and get what you need; circle your response):

\begin{tabular}{|c|c|c|c|c|}
\hline None at all & A little bit & Some & $\begin{array}{l}\text { A good } \\
\text { amount }\end{array}$ & A lot \\
\hline 1 & 2 & 3 & 4 & 5 \\
\hline
\end{tabular}


12. Please rate on a scale from 1 to 5 how much confidence you have in your ability to manage your own mental health and wellbeing (circle your response):

\begin{tabular}{|c|c|c|c|c|}
\hline None at all & A little bit & Some & $\begin{array}{l}\text { A good } \\
\text { amount }\end{array}$ & A lot \\
\hline 1 & 2 & 3 & 4 & 5 \\
\hline
\end{tabular}




\section{Appendix $M$}

\section{Post Wellbeing Activity Evaluation Questions}

1. What did you like about this activity?

2. What would you change about this activity?

3. What should be added to this activity to help students learn and use the knowledge learned from the activity?

4. Was there any information that you would have liked to learn about, that we didn't cover? If so, what would you have liked to learn about?

5. Was the method through which the information was delivered acceptable to you (e.g., through a sharing circle and interactive?) How would you like to see it changed?

6. Were the questions asked of you before and after the activity acceptable to you? How would you like to see them changed? 


\section{Knowledge to Action Interview Guide}

\section{Appendix $N$}

Knowledge Uptake and Retention (since a month ago)

1. Was there anything about mental health and wellbeing that you learned from the activity? If so, what did you learn?

2. Did you learn anything from the wellbeing activity about resources people can access if they suffer from a mental health concern? If so, what did you learn?

3. Did you learn anything from the wellbeing activity about how to get information about mental health and wellbeing? If so, what did you learn?

4. Did you gain any knowledge about coping strategies that people can use to manage their mental health concerns? If so, what did you learn?

5. Please rate on a scale from 1 to 5 how much mental health knowledge you currently remember since the wellbeing activity:

\begin{tabular}{|c|c|c|c|c|}
\hline None at all & A little bit & Some & $\begin{array}{l}\text { A good } \\
\text { amount }\end{array}$ & A lot \\
\hline 1 & 2 & 3 & 4 & 5 \\
\hline
\end{tabular}

6. If they responded no to question 1 to 4 and a low score on question 5: How could the activity be changed to be beneficial to you/help you learn? 
7. Please rate on a scale from 1 to 5 how much confidence you have in managing your own mental health and wellbeing as a result of the wellbeing activity:

\begin{tabular}{|c|c|c|c|c|}
\hline None at all & A little bit & Some & $\begin{array}{l}\text { A good } \\
\text { amount }\end{array}$ & A lot \\
\hline 1 & 2 & 3 & 4 & 5 \\
\hline
\end{tabular}

Knowledge to Action

8. If they responded yes to questions 1 to 3: Have you used anything that you learned from the wellbeing activity in your daily life?

If yes, what have you used and how?

How often do you use such knowledge (e.g., once per day, once per week, etc.)?

9. Please rate on a scale from 1 to 5 how much the strategies learned through the activity has helped you manage your own wellbeing:

\begin{tabular}{|c|c|c|c|c|}
\hline None at all & A little bit & Some & $\begin{array}{l}\text { A good } \\
\text { amount }\end{array}$ & A lot \\
\hline 1 & 2 & 3 & 4 & 5 \\
\hline
\end{tabular}

10. If no to question 8: Do you plan to use anything that you learned from the wellbeing activity in your daily life?

If yes, what do you plan to use and how?

If no, why not? 
11. This project was about Aboriginal youth knowledge about mental health and wellbeing. For you, what do you think is the biggest take home message from the entire project?

12. How do you perceive the current state of Aboriginal youth knowledge about mental health and wellbeing?

13. What do you think the future holds for Aboriginal youth knowledge about mental health and wellbeing? How do you think it could be promoted and fostered? 


\section{Appendix $O$}

\section{Reconsent Agreement: Knowledge Translation Strategy}

\section{RYERSONUNIVERSITY}

\section{Re-Consent Agreement Knowledge Translation Strategy}

You previously signed a consent agreement to participate in the wellbeing activity and follow-up interview portion of this project. It has come to my attention that we did not include information about hiring community members for the purpose of data analysis and interpretation in the previous consent form that you signed. I am hoping to hire a community member to assist me with the data analysis and interpretation in order to provide them with valuable research experiences. This community member would also provide me with important insight and perspectives that I may not have otherwise considered throughout the analysis in order to determine in the most accurate and representative results from this study.

Hiring community members for help with various aspects of the project is very commonly done in an Aboriginal context. If a community member is hired, they will be working with your deidentified data. They will also be required to sign a confidentiality agreement, which means that they will not be able to disclose any participant information to anyone.

If you have any questions about this, please ask me now or you may contact me at a later time.

Caitlin Davey, M.A.

Ph.D. Student

Department of Psychology

Ryerson University

350 Victoria Street

Toronto Ontario Canada M5B 2K3

Phone: 416-979-5000, ext 2188 (after pressing 1)

Email: cdavey@psych.ryerson.ca

If you have questions regarding your rights as a human subject and participant in this study, you may contact the Ryerson University Research Ethics Board for information.

Dr. Lynn Lavallee

Research Ethics Board

c/o Office of the Vice President, Research and Innovation

Ryerson University 


\section{Victoria Street}

Toronto, ON M5B 2K3

Phone: 4169795000 ext. 4791

Email: lavallee@ryerson.ca

\section{Agreement:}

Your signature below indicates that you have read the information in this agreement and have had a chance to ask any questions about the hiring of a community member to analyze and interpret your de-identified data. You have been told that by signing this consent agreement you are not giving up any of your legal rights.

Name of Participant (please print)

Signature of Participant

Date

Signature of Investigator

Date 
Appendix $P$
Consent Agreement: Key Informant Interviews

\section{RYERSONUNIVERSITY}

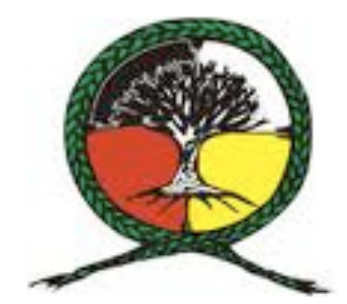

\section{Consent Agreement Key Informant Interviews}

You are being asked to participate in a research study. Before you give your consent to be a volunteer, it is important that you read the following information and ask as many questions as necessary to be sure you understand what you will be asked to do.

\section{Investigators:}

Caitlin Davey, M.A.

Ph.D. Student

Department of Psychology

Ryerson University

(supervised by Kelly McShane)

\author{
Kelly McShane, Ph.D., C. Psych. \\ Assistant Professor \\ Department of Psychology \\ Ryerson University \\ 350 Victoria Street \\ Toronto, ON, M5B 2K3 \\ 416-979-5000 ext. 2051 \\ kmcshane@psych.ryerson.ca
}

\section{Community Partner:}

The investigators have partnered with Ryerson Aboriginal Student Services (RASS) to complete this study. This means that RASS has identified the aims of the present study as a need for their organization and will collaborate with the research team regarding all aspects of the study (i.e., from research design to the interpretation of analysis). Ruth Koleszar-Green, BSW, MSW, RSW, is the Aboriginal Academic Support Advisor at RASS and is the RASS representative with which the investigators will be working to complete the present study. RASS staff members, including Ruth Koleszar-Green, will not have access to confidential information expressed by participants in the present study.

\section{Purpose of the Study:}

The purpose of this project is to understand Aboriginal perspectives of mental health, gaps in mental health knowledge among Aboriginal students, and the best ways to give Aboriginal students mental health knowledge. The results of the study aims to increase the understanding of mental health concerns among Aboriginal students and increase Aboriginal students' mental health knowledge and resources for coping with mental health concerns. 
Description of the Study: This study is being completed for a psychology graduate student thesis project. As part of this study, you will be asked to participate in an individual interview, where you will answer some questions about the project, as you have been involved at various points in the study. The interview will be approximately 30 to 45 minutes and will take place in a private and comfortable room at Ryerson Aboriginal Student Services.

The only experimental aspect of this study is the gathering of information for the purpose of analysis.

Risks or Discomforts: It is possible that during this study you will become uncomfortable. During the interview, you may feel uncomfortable with topics that we may discuss or feel self conscious about your knowledge. If you begin to feel uncomfortable, you may discontinue participating. You may also discontinue participating for any other reason. In addition, questions addressing participants personal experiences with mental health will be not be asked.

Benefits of the Study: You may receive some benefits from being in this study. The results of this study may help you better understand wellbeing and increase your wellbeing. We cannot guarantee, however, that you will receive any benefits from participating in this study.

Confidentiality: Confidentiality will be respected and no information that discloses your identity will be released or published without your consent, unless required by law. The research team will maintain confidentiality. This research team includes Dr. Kelly McShane, Caitlin Davey and possibly a member of your community in order to help analyze the data and build capacity. Any community members who are hired will sign a confidentiality agreement and will work with de-identified data. Direct quotations will be used in the publication of the data collected; however, all identifying information will be removed from the quotations.

If you do not want your quotations to be used in any publications, please let the researchers know and this will be documented and respected.

I agree to have my direct quotations used

I do not agree to have my direct quotations used, but agree that what I say can be paraphrased I do not agree to have my direct quotations used or paraphrased

The results of the study will be made available to all participants before publication, so they have a chance to make changes to anything (including quotes) that they are uncomfortable with.

The activity and interview will be audio taped. The digital audio files will be kept on a secure server at Ryerson University and will be password protected. No identifying information will be tied to the files or transcripts. Audio files will be destroyed after we have confirmed all vital information (2-3 years). After this time, data (transcripts) will be transferred to RASS where they will be stored (without any identifying information tied to transcripts). At this point, only study staff will have access to these data (i.e., the principal investigators and RASS). After 10 years, all information will be destroyed.

\section{Incentive to Participate}


You will be paid $\$ 10$ total for your participation in the interview.

Voluntary Nature of Participation: Participation in this study is voluntary. Your choice of whether or not to participate will not influence your future relations with Ryerson University or RASS. If you decide to participate, you are free to withdraw your consent and to stop your participation at any time without penalty or loss of benefits to which you are allowed. You are also allowed to withdraw your data at a later time if you so choose.

At any particular point in the study, you may refuse to answer any particular question or stop participation altogether.

Questions about the Study: If you have any questions about the research now, please ask. If you have questions later about the research, you may contact.

Caitlin Davey, M.A.

Ph.D. Student

Department of Psychology

Ryerson University

350 Victoria Street

Toronto Ontario Canada M5B 2K3

Phone: 416-979-5000, ext 2188 (after pressing 1)

Email: cdavey@psych.ryerson.ca

If you have questions regarding your rights as a human subject and participant in this study, you may contact the Ryerson University Research Ethics Board for information.

Dr. Lynn Lavallee

Research Ethics Board

c/o Office of the Vice President, Research and Innovation

Ryerson University

350 Victoria Street

Toronto, ON M5B 2K3

Phone: 4169795000 ext. 4791

Email: lavallee@ryerson.ca

\section{Agreement:}

Your signature below indicates that you have read the information in this agreement and have had a chance to ask any questions you have about the study. Your signature also indicates that you agree to be in the study and have been told that you can change your mind and withdraw your consent to participate at any time. You have been given a copy of this agreement.

You have been told that by signing this consent agreement you are not giving up any of your legal rights.

Name of Participant (please print)

Signature of Participant

Date 
Your signature below indicates that you have read and understand that you will be audiorecorded for only the purposes of this study. Your signature indicates that you agree to be audio-recorded and have been told that you can change your mind and withdraw this consent to be audio-recorded at any time.

Name of Participant (please print)

Signature of Participant

Signature of Investigator

\section{Date}

Date 
Key Informant Interview Questions

\section{Appendix $Q$}

1. This project developed a wellbeing activity based on students' preferences and this activity was completed and evaluated.

a. For those who did not attend the activity: Have you heard from students or staff that might give you any insight regarding how the activity went/how students responded to the activity? (Do you think it enhanced knowledge, changed perceptions/assumptions/definitions of mental health for Aboriginal students?)

b. For those who attended the activity: What did you think of the activity (e.g., what did you like, what did you not like, what would you change and what would you keep the same? Do you think it enhanced knowledge, changed perceptions/assumptions/definitions of mental health for Aboriginal students?)

2. How do you see your role in this project?

3. For you, what are the key take away messages from this project?

4. Where do you think we are at with the current state of student mental health knowledge? Where do you think we are going? What does the future hold?

5. I asked students follow-up questions about the wellbeing activity, but considered bringing someone else in to ask these questions in case students might feel more comfortable providing feedback. What impact, if any, was there in having me evaluate the project? What impact would there have been if someone else were asked to do the follow-up interviews?

Probes: How comfortable do you think people would be providing candid feedback to me?

What does anonymity, confidentiality, objectivity, etc mean to you and how relevant do you think they are to this project?

6. This project was designed to address a stated need within the community partner, do you think that this project addressed any needs within this community and if yes, how so? 
Appendix $R$

Final Tool Development Phase Codebook

\begin{tabular}{|c|c|c|c|c|}
\hline Code Name & Definition & When to use Code & When not to use code & Examples \\
\hline \multicolumn{5}{|c|}{ What is Mental Health? } \\
\hline MH General & $\begin{array}{l}\text { When participants talk } \\
\text { about their general } \\
\text { thoughts on mental } \\
\text { health (i.e., when they } \\
\text { list certain things } \\
\text { without going into much } \\
\text { detail about them) or say } \\
\text { the first things that come } \\
\text { to mind }\end{array}$ & $\begin{array}{l}\text { When people are } \\
\text { providing one-word } \\
\text { answers or the first thing } \\
\text { that comes to mind to } \\
\text { the question: what is } \\
\text { mental health? This can } \\
\text { includes technical and } \\
\text { scientific terms and } \\
\text { description words }\end{array}$ & $\begin{array}{l}\text { Do not use this code } \\
\text { when someone talks } \\
\text { about something that } \\
\text { would go in any of the } \\
\text { codes below }\end{array}$ & $\begin{array}{l}\text { "I think of like } \\
\text { counsellors, } \\
\text { psychologists, social } \\
\text { works, I also think of } \\
\text { patients, people } \\
\text { suffering from mental } \\
\text { illnesses, I think of } \\
\text { homeless people." }\end{array}$ \\
\hline MH Wholistic & $\begin{array}{l}\text { When participants talk } \\
\text { about the medicine } \\
\text { wheel or wholism or } \\
\text { physical, emotional, } \\
\text { spiritual, and mental } \\
\text { health's when referring } \\
\text { to mental health, and as } \\
\text { part of the whole person }\end{array}$ & $\begin{array}{l}\text { Keywords: medicine } \\
\text { wheel, wholistic, } \\
\text { wholism, whole person, } \\
\text { mental, physical, } \\
\text { emotional, spiritual, } \\
\text { balance, }\end{array}$ & $\begin{array}{l}\text { When participants are } \\
\text { talking about this themes } \\
\text { when discussing } \\
\text { something other than } \\
\text { mental health and when } \\
\text { discussing wholism as a } \\
\text { cultural difference } \\
\text { between Aboriginal and } \\
\text { Western worldviews }\end{array}$ & $\begin{array}{l}\text { "It's like I'm not sure if } \\
\text { like um, if enough, if } \\
\text { enough attention is being } \\
\text { paid to the holistic part } \\
\text { other than just kind of } \\
\text { the like the tip of the hat } \\
\text { tribute to it. But it's like } \\
\text { where is the actual like } \\
\text { work being done when } \\
\text { they're tying them } \\
\text { together to show that } \\
\text { they are connected, you } \\
\text { know?" }\end{array}$ \\
\hline MH Relational & When participants talk & & Do not use when & "Young Blood has a \\
\hline
\end{tabular}




\begin{tabular}{|c|c|c|c|c|}
\hline & $\begin{array}{l}\text { (i.e., it is passed down } \\
\text { from generation to } \\
\text { generation) }\end{array}$ & & about something cultural & $\begin{array}{l}\text { that survivor guilt is } \\
\text { passed down from } \\
\text { indigenous body to } \\
\text { indigenous body and the } \\
\text { beliefs in blood memory } \\
\text { and the trauma and the } \\
\text { PTSD has been almost } \\
\text { embedded in our blood } \\
\text { memory." }\end{array}$ \\
\hline MH Cultural Connection & $\begin{array}{l}\text { When participants talk } \\
\text { about how cultural } \\
\text { connection, } \\
\text { reconnection, or } \\
\text { disconnection is } \\
\text { important to mental } \\
\text { health and wellbeing as } \\
\text { well as how cultural } \\
\text { connection, } \\
\text { reconnections, and } \\
\text { disconnection can } \\
\text { improve/or not improve } \\
\text { mental health }\end{array}$ & $\begin{array}{l}\text { Keywords: ceremonies, } \\
\text { spirituality } \\
\text { Can also use this code if } \\
\text { people are saying that } \\
\text { cultural connection was } \\
\text { not helpful for them and } \\
\text { if saying that cultural } \\
\text { disconnection was } \\
\text { helpful for them }\end{array}$ & $\begin{array}{l}\text { Do not use this code } \\
\text { when participants are } \\
\text { talking about the } \\
\text { differences between } \\
\text { Aboriginal and Western } \\
\text { worldviews on mental } \\
\text { health, how they balance } \\
\text { the two or use both in } \\
\text { their understand, as this } \\
\text { would go under MH } \\
\text { Cultural Differences } \\
\\
\text { Do not use when } \\
\text { participants are talking } \\
\text { about coping strategies }\end{array}$ & $\begin{array}{l}\text { "When you are divorced } \\
\text { from the land and } \\
\text { divorced from your } \\
\text { culture and your } \\
\text { teachings and all those } \\
\text { spaces, those are not } \\
\text { going to be spaces of } \\
\text { strength." }\end{array}$ \\
\hline MH Stigma & $\begin{array}{l}\text { When participants talk } \\
\text { about mental health as } \\
\text { being stigmatized or } \\
\text { how they were } \\
\text { embarrassed or reluctant } \\
\text { to get help or admit their }\end{array}$ & $\begin{array}{l}\text { Keywords: Stigma. } \\
\text { labels, stereotypes, } \\
\text { embarrassment, people } \\
\text { misunderstanding what } \\
\text { people with mental } \\
\text { health are going through }\end{array}$ & $\begin{array}{l}\text { Do not use if } \\
\text { participants are talking } \\
\text { about how to reduce the } \\
\text { stigma around mental } \\
\text { health because this } \\
\text { would be coded under }\end{array}$ & $\begin{array}{l}\text { "I also think it's a lot of } \\
\text { stigma" }\end{array}$ \\
\hline
\end{tabular}




\begin{tabular}{|c|c|c|c|c|}
\hline & issues because of stigma & & $\begin{array}{l}\text { "MH Gaps" or } \\
\text { "Answers MH Gaps" }\end{array}$ & \\
\hline MH Cultural Differences & $\begin{array}{l}\text { When participants } \\
\text { discuss differences } \\
\text { between Western ways } \\
\text { of viewing mental health } \\
\text { and Aboriginal ways of } \\
\text { viewing mental health, } \\
\text { how they conflict with } \\
\text { one another, how they } \\
\text { balance the two and/or } \\
\text { how they use both to } \\
\text { understand mental } \\
\text { health. }\end{array}$ & $\begin{array}{l}\text { When participants talk } \\
\text { about disadvantages that } \\
\text { Aboriginal people have } \\
\text { faced that impact their } \\
\text { mental health status as } \\
\text { well as differences in } \\
\text { views on mental health, } \\
\text { which can include } \\
\text { differences in views } \\
\text { from mainstream } \\
\text { services that they } \\
\text { accessed }\end{array}$ & $\begin{array}{l}\text { Do not use when talking } \\
\text { about how coping styles } \\
\text { may be different for } \\
\text { Aboriginal versus non- } \\
\text { Aboriginal people as this } \\
\text { would go under the } \\
\text { "Coping Differences" } \\
\text { code. }\end{array}$ & $\begin{array}{l}\text { "I guess the correct term } \\
\text { would be environmental } \\
\text { factors. You think about } \\
\text { where our people have } \\
\text { had to work and the fact } \\
\text { that they probably } \\
\text { weren't given the } \\
\text { information to do that } \\
\text { work safely. Anybody in } \\
\text { the boating industry, the } \\
\text { asbestos that was there, } \\
\text { or you think about } \\
\text { anyone who worked in } \\
\text { logging, the physical } \\
\text { ailments that come with } \\
\text { that." }\end{array}$ \\
\hline MH Identity & $\begin{array}{l}\text { When participants talk } \\
\text { about their identity in } \\
\text { relation to their mental } \\
\text { health/wellbeing }\end{array}$ & $\begin{array}{l}\text { When participants talk } \\
\text { about learning or not } \\
\text { knowing their heritage } \\
\text { language or use the word } \\
\text { "identity" when } \\
\text { discussing mental health }\end{array}$ & $\begin{array}{l}\text { When participants are } \\
\text { talking about cultural } \\
\text { differences or culture } \\
\text { more generally }\end{array}$ & $\begin{array}{l}\text { Ya, and I would say I } \\
\text { have the exact same } \\
\text { thing going on, ya. It's } \\
\text { kind of like okay, what } \\
\text { am I? So ya, Identity is a } \\
\text { huge thing, for sure. }\end{array}$ \\
\hline MH Understanding & $\begin{array}{l}\text { When participants talk } \\
\text { about how they } \\
\text { developed their } \\
\text { understanding of mental } \\
\text { health }\end{array}$ & $\begin{array}{l}\text { This can include them } \\
\text { talking about their own } \\
\text { experiences with mental } \\
\text { health and experiences } \\
\text { with friends and family } \\
\text { members with mental }\end{array}$ & & $\begin{array}{l}\text { "Sometimes I get like } \\
\text { that too. I'll like get high } \\
\text { anxiety and I'll get panic } \\
\text { attacks sometimes but I } \\
\text { have to be really stressed } \\
\text { out to that point you } \\
\text { know? I think stress has }\end{array}$ \\
\hline
\end{tabular}




\begin{tabular}{|c|c|c|c|c|}
\hline & & $\begin{array}{l}\text { health issues } \\
\text { Try to code the people } \\
\text { or situations through } \\
\text { which they learned } \\
\text { about mental health } \\
\text { under this code, but if } \\
\text { the actual understanding } \\
\text { of mental health goes } \\
\text { under another code } \\
\text { (e.g., when they talk } \\
\text { about their wholistic } \\
\text { understanding this } \\
\text { would go under MH } \\
\text { Wholistic) use the } \\
\text { corresponding code }\end{array}$ & & $\begin{array}{l}\text { a lot to do with it too } \\
\text { when you really come } \\
\text { down to it. A lot of } \\
\text { people don't recognize } \\
\text { that, they don't see how } \\
\text { stress can be a mental } \\
\text { illness or trigger a } \\
\text { mental illness or } \\
\text { snowball into one." }\end{array}$ \\
\hline MH Miscellaneous & $\begin{array}{l}\text { When participants talk } \\
\text { about something that } \\
\text { doesn't fit under the } \\
\text { above codes }\end{array}$ & & & $\begin{array}{l}\text { "It's an evolving } \\
\text { definition" }\end{array}$ \\
\hline \multicolumn{5}{|c|}{ How would you cope with your mental health concerns? } \\
\hline Coping General & $\begin{array}{l}\text { When participants talk } \\
\text { about the different ways } \\
\text { that they cope with their } \\
\text { mental health concerns } \\
\text { and how it was helpful }\end{array}$ & $\begin{array}{l}\text { Keywords: Talking, } \\
\text { ceremony, searching } \\
\text { Internet }\end{array}$ & $\begin{array}{l}\text { Note: Participants may } \\
\text { talk about this in other } \\
\text { areas of the focus group, } \\
\text { other than just when I } \\
\text { ask } \\
\text { Do not use if they list }\end{array}$ & $\begin{array}{l}\text { "Talk to friends because } \\
\text { at the end of the day } \\
\text { you're lucky if you have } \\
\text { your parents, but like } \\
\text { your circle of friends is } \\
\text { also very important in } \\
\text { your life so if their in the } \\
\text { know and they know } \\
\text { what's going on with }\end{array}$ \\
\hline
\end{tabular}




\begin{tabular}{|c|c|c|c|c|}
\hline & & & $\begin{array}{l}\text { mental health resources } \\
\text { because this would go } \\
\text { under the "MH } \\
\text { Resources" code }\end{array}$ & $\begin{array}{l}\text { you so if you're acting a } \\
\text { little weird and they } \\
\text { notice then they can do } \\
\text { their best to help you } \\
\text { out" }\end{array}$ \\
\hline $\begin{array}{l}\text { Coping Cultural } \\
\text { Differences }\end{array}$ & $\begin{array}{l}\text { When participants talk } \\
\text { about how coping styles } \\
\text { may differ between } \\
\text { Aboriginal and Western } \\
\text { contexts }\end{array}$ & $\begin{array}{l}\text { Can use when } \\
\text { participants explicitly } \\
\text { talk about how their } \\
\text { coping style is different } \\
\text { from the Western way or } \\
\text { when they list something } \\
\text { that is clearly from } \\
\text { Aboriginal culture } \\
\text { Keywords: sweats, } \\
\text { smudging, talk to elder }\end{array}$ & & $\begin{array}{l}\text { "The way they dealt } \\
\text { with it through their } \\
\text { doctors was through } \\
\text { drugs and prescriptions } \\
\text { or whatever whereas you } \\
\text { know maybe in an } \\
\text { Aboriginal context at } \\
\text { least in my experience } \\
\text { back in my area it is } \\
\text { dealt with it more in the } \\
\text { family so there is a } \\
\text { different way of dealing } \\
\text { with it but here it's like } \\
\text { you go to the doctor get } \\
\text { some treatment" }\end{array}$ \\
\hline \multicolumn{5}{|c|}{ If you needed to get help for a mental health problem, where would you go? } \\
\hline MH Help & $\begin{array}{l}\text { When participants talk } \\
\text { about where they would } \\
\text { go to get help if they } \\
\text { needed it. } \\
\text { This code will also apply } \\
\text { when participants } \\
\text { (staff/faculty) talk about } \\
\text { the details of services }\end{array}$ & $\begin{array}{l}\text { Keywords: Any services } \\
\text { they list } \\
\text { You can also code } \\
\text { anything participants } \\
\text { say about how helpful } \\
\text { the resources were or } \\
\text { issues with the resource } \\
\text { and details about their }\end{array}$ & $\begin{array}{l}\text { Do not use when they } \\
\text { list internal coping } \\
\text { strategies, as these } \\
\text { would be coded under } \\
\text { the "General Coping" } \\
\text { code } \\
\text { Do not use when } \\
\text { discussing where they }\end{array}$ & $\begin{array}{l}\text { "I would talk to } \\
\text { someone from RASS" } \\
\text { "Not all aboriginal } \\
\text { spaces... due to the } \\
\text { impact of colonization, } \\
\text { so many are still } \\
\text { struggling with being } \\
\text { good enough to try to }\end{array}$ \\
\hline
\end{tabular}




\begin{tabular}{|c|c|c|c|c|}
\hline & they provide for students & $\begin{array}{l}\text { experiences with the } \\
\text { resource, but be sure to } \\
\text { code the name of the } \\
\text { service they list }\end{array}$ & $\begin{array}{l}\text { would go to get mental } \\
\text { health information }\end{array}$ & $\begin{array}{l}\text { exist that they're } \\
\text { adhering to western } \\
\text { understandings of how } \\
\text { they should be engaging } \\
\text { and not..." }\end{array}$ \\
\hline \multicolumn{5}{|c|}{ What would you like to know more about mental health? } \\
\hline $\begin{array}{l}\text { MH Knowledge Gaps } \\
\text { General }\end{array}$ & $\begin{array}{l}\text { When participants talk } \\
\text { about gaps in their } \\
\text { mental health knowledge } \\
\text { or what they would want } \\
\text { to know more about }\end{array}$ & $\begin{array}{l}\text { Keywords: Why...? I } \\
\text { want to understand..., } \\
\text { how come...? }\end{array}$ & & $\begin{array}{l}\text { "And like why is there } \\
\text { stigma? Like why do we } \\
\text { have to stigmatized } \\
\text { things that we don't } \\
\text { understand?" }\end{array}$ \\
\hline $\begin{array}{l}\text { MH Knowledge Gaps } \\
\text { System }\end{array}$ & $\begin{array}{l}\text { When participants talk } \\
\text { specifically about } \\
\text { wanting to know more } \\
\text { about navigating the } \\
\text { mental health system } \\
\text { (i.e., what is out there, } \\
\text { how do I get a } \\
\text { counsellor?, what is } \\
\text { covered versus not } \\
\text { covered?) }\end{array}$ & & & $\begin{array}{l}\text { how to like seek out } \\
\text { treatment or support or I } \\
\text { don't know like and also } \\
\text { what to look for you } \\
\text { know because it's one } \\
\text { thing to just say go to } \\
\text { your doctor go to the } \\
\text { nearest therapist you } \\
\text { know but what if that's a } \\
\text { bad therapist. What's } \\
\text { good treatment? What } \\
\text { are the different types of } \\
\text { treatment out there? }\end{array}$ \\
\hline Answers MH Gaps & $\begin{array}{l}\text { When a participant talks } \\
\text { about a potential } \\
\text { solution to one of the } \\
\text { questions someone } \\
\text { raised about what they } \\
\text { would like to know more }\end{array}$ & $\begin{array}{l}\text { Examples might be ways } \\
\text { we can fight back } \\
\text { against stigmas and } \\
\text { ways we can increase } \\
\text { culturally appropriate } \\
\text { services and emphasize }\end{array}$ & & $\begin{array}{l}\text { "Yeah, it's to resist and } \\
\text { it's full of risks. I'm } \\
\text { willing to take the risks } \\
\text { because I feel great } \\
\text { people around me that } \\
\text { are willing to take that }\end{array}$ \\
\hline
\end{tabular}




\begin{tabular}{|c|c|c|c|c|}
\hline & about mental health & $\begin{array}{l}\text { Aboriginal worldviews } \\
\text { more in services and } \\
\text { organizations }\end{array}$ & & $\begin{array}{l}\text { risk with me and I'm not } \\
\text { alone. But then, what if } \\
\text { you are? What if you are } \\
\text { the only one? Or what if } \\
\text { I happen to be in a } \\
\text { situation where, really } \\
\text { for all intense } \\
\text { purposes... I'm not } \\
\text { going to have any } \\
\text { negative ramifications, I } \\
\text { can't lose, but what if } \\
\text { you are in a situation } \\
\text { where you do have an } \\
\text { awful lot to lose? How } \\
\text { then do we support and } \\
\text { help... I don't know." }\end{array}$ \\
\hline \multicolumn{5}{|c|}{ If you wanted to get mental health information, how/where would you get it? } \\
\hline MH Info Services & $\begin{array}{l}\text { When participants talk } \\
\text { about the services from } \\
\text { which they would get } \\
\text { mental health } \\
\text { information }\end{array}$ & $\begin{array}{l}\text { Keywords: Any services } \\
\text { they may list when } \\
\text { talking about where to } \\
\text { get mental health } \\
\text { information }\end{array}$ & $\begin{array}{l}\text { Do not code when they } \\
\text { list an internal coping } \\
\text { strategy or when they } \\
\text { talk about where they } \\
\text { would go to address a } \\
\text { mental health problem }\end{array}$ & "A helpline" \\
\hline MH Info People & $\begin{array}{l}\text { When participants talk } \\
\text { about the people from } \\
\text { which they would get } \\
\text { mental health } \\
\text { information }\end{array}$ & $\begin{array}{l}\text { Any people they may list } \\
\text { when talking about } \\
\text { where to get mental } \\
\text { health information (can } \\
\text { include psychologists, } \\
\text { counsellors, etc.) }\end{array}$ & $\begin{array}{l}\text { Do not code when they } \\
\text { list an internal coping } \\
\text { strategy or when they } \\
\text { talk about where they } \\
\text { would go to address a } \\
\text { mental health problem }\end{array}$ & $\begin{array}{l}\text { "My friend because she } \\
\text { works in the area of } \\
\text { mental health" }\end{array}$ \\
\hline MH Info Internet & When participants talk & Any website may list & Do not code when they & "The Internet" \\
\hline
\end{tabular}




\begin{tabular}{|c|c|c|c|c|}
\hline & $\begin{array}{l}\text { about internet resources } \\
\text { from which they could } \\
\text { get mental health } \\
\text { information }\end{array}$ & $\begin{array}{l}\text { when talking about } \\
\text { where to get mental } \\
\text { health information }\end{array}$ & $\begin{array}{l}\text { list an internal coping } \\
\text { strategy or when they } \\
\text { talk about where they } \\
\text { would go to address a } \\
\text { mental health problem. }\end{array}$ & \\
\hline \multicolumn{5}{|c|}{ What is the best way to communicate mental health information to Aboriginal students? } \\
\hline KT Method & $\begin{array}{l}\text { When participants talk } \\
\text { about the best ways to } \\
\text { communicate mental } \\
\text { health knowledge to } \\
\text { Aboriginal students }\end{array}$ & $\begin{array}{l}\text { Keywords: Anything } \\
\text { they list regarding how it } \\
\text { should be done, such as } \\
\text { in activity format, in a } \\
\text { circle, and with } \\
\text { traditional ceremonies, } \\
\text { etc. }\end{array}$ & $\begin{array}{l}\text { Do not use if in } \\
\text { reference to who should } \\
\text { facilitate the activity and } \\
\text { the content of the } \\
\text { activity }\end{array}$ & $\begin{array}{l}\text { "Activity. If this was a } \\
\text { lecture style I wouldn't } \\
\text { be here" }\end{array}$ \\
\hline KT Content & $\begin{array}{l}\text { When participants talk } \\
\text { about any mental health } \\
\text { content that should be } \\
\text { communicated to } \\
\text { Aboriginal students }\end{array}$ & $\begin{array}{l}\text { Keywords: Anything } \\
\text { they list regarding } \\
\text { content such as } \\
\text { information on different } \\
\text { disorders, how to better } \\
\text { support people with } \\
\text { mental health problems }\end{array}$ & $\begin{array}{l}\text { Do not use if in } \\
\text { reference to who should } \\
\text { facilitate the activity or } \\
\text { the method of } \\
\text { communication. Do not } \\
\text { use if they talk about } \\
\text { what they want to know } \\
\text { if reference to gaps in } \\
\text { knowledge - use one of } \\
\text { the two knowledge gaps } \\
\text { code }\end{array}$ & $\begin{array}{l}\text { "I would like some } \\
\text { information on } \\
\text { schizophrenia" }\end{array}$ \\
\hline KT Facilitator/Presenter & $\begin{array}{l}\text { When participants talk } \\
\text { about who would be best } \\
\text { to facilitate this activity } \\
\text { or present/provide } \\
\text { information during the } \\
\text { activity }\end{array}$ & $\begin{array}{l}\text { Keywords: Anything } \\
\text { they regarding who } \\
\text { should facilitate the } \\
\text { activity such as Elders, } \\
\text { social workers, } \\
\text { storytellers etc. }\end{array}$ & $\begin{array}{l}\text { Do not use if in } \\
\text { reference to the content } \\
\text { of the activity or the } \\
\text { method of the activity }\end{array}$ & $\begin{array}{l}\text { "I think elders should } \\
\text { help facilitate it because } \\
\text { they have important } \\
\text { traditional knowledge } \\
\text { that could be helpful for } \\
\text { Aboriginal students" }\end{array}$ \\
\hline
\end{tabular}




\begin{tabular}{|l|l|l|l|l|}
\hline $\begin{array}{l}\text { General } \\
\text { Recommendations }\end{array}$ & $\begin{array}{l}\text { When someone makes a } \\
\text { recommendation about } \\
\text { the system or needs in } \\
\text { Aboriginal communities }\end{array}$ & $\begin{array}{l}\text { Notes: We may not end } \\
\text { up using this data for in } \\
\text { the results, but it may be } \\
\text { helpful to code it for } \\
\text { now, just in case }\end{array}$ & $\begin{array}{l}\text { "And then you know, } \\
\text { that happens a lot but } \\
\text { there needs to be a } \\
\text { different system set up } \\
\text { for Aboriginal people } \\
\text { and the Aboriginal } \\
\text { perspective" }\end{array}$ \\
\hline Miscellaneous & $\begin{array}{l}\text { Anything that doesn't fit } \\
\text { into any of the codes } \\
\text { listed above }\end{array}$ & & \\
\hline
\end{tabular}

\section{Guidelines to keep in mind when coding:}

(1) You don't have to code everything

(2) You can code what the interview (I) says when I am contributing to the question, but not when I am just repeating what people are saying and not when I am asking questions

(3) Be sure to include the Is, P1s, P2s, etc when you code

(4) Keep track of questions or uncertainties when you code so that we can clarify them when we chat next

(5) Try not to code identifying information

Note: Some of these codes have changed since writing the results section. Therefore, although this is the final codebook use, it is not the final list of codes/themes. 
Knowledge Translation Activity Evaluation Codebook

\begin{tabular}{|c|c|c|c|c|}
\hline Code Name & Definition & When to use Code & When not to use code & Examples \\
\hline \multicolumn{5}{|c|}{ What did you like about the activity (includes what they liked about the method)? Merged Q1 and Q5 } \\
\hline Sense of Community & $\begin{array}{l}\text { When participants talk } \\
\text { about anything that has } \\
\text { to do with being with } \\
\text { other Aboriginal people, } \\
\text { if they specifically note } \\
\text { that they liked the } \\
\text { community feel }\end{array}$ & $\begin{array}{l}\text { Keywords: Community, } \\
\text { Aboriginal people, } \\
\text { sharing space with other } \\
\text { Aboriginal people }\end{array}$ & $\begin{array}{l}\text { When an Aboriginal } \\
\text { community member is } \\
\text { not identified or the term } \\
\text { community is not used }\end{array}$ & $\begin{array}{l}\text { "I liked the community } \\
\text { feeling" } \\
\text { "To share spaces and } \\
\text { ideas with other } \\
\text { Aboriginal people" } \\
\text { "I needed to be among } \\
\text { healers" }\end{array}$ \\
\hline General Likes & $\begin{array}{l}\text { When participants talk } \\
\text { what they generally } \\
\text { liked about the activity } \\
\text { and can sometimes be } \\
\text { found under the methods } \\
\text { question }\end{array}$ & Keywords: like, good & $\begin{array}{l}\text { If participants talk about } \\
\text { wanting more interactive } \\
\text { elements, then code this } \\
\text { under the Participation/ } \\
\text { Engagement code }\end{array}$ & $\begin{array}{l}\text { "Inviting and } \\
\text { welcoming" } \\
\text { "It was good, good } \\
\text { refreshments, and } \\
\text { honorarium" }\end{array}$ \\
\hline \multicolumn{5}{|c|}{ What would you change, add to, or liked to have learned in this activity? (Merged Q2, Q3, \& Q4) } \\
\hline $\begin{array}{l}\text { Participation/ } \\
\text { Engagement }\end{array}$ & $\begin{array}{l}\text { When participants talk } \\
\text { about more participation } \\
\text { or discussion from } \\
\text { participants, focused } \\
\text { discussions, stimulate } \\
\text { more engaging } \\
\text { discussions }\end{array}$ & $\begin{array}{l}\text { Keywords: Discussions, } \\
\text { engaging, focus, } \\
\text { participate, students }\end{array}$ & $\begin{array}{l}\text { When the amount of } \\
\text { student participation or } \\
\text { engagement is not } \\
\text { mentioned }\end{array}$ & $\begin{array}{l}\text { "More student } \\
\text { participation instead of } \\
\text { professionals } \\
\text { monopolizing the } \\
\text { discussion, although } \\
\text { their input was definitely } \\
\text { needed and appreciated, } \\
\text { it felt like the students }\end{array}$ \\
\hline
\end{tabular}




\begin{tabular}{|c|c|c|c|c|}
\hline & & & & were a low priority" \\
\hline General Topics & $\begin{array}{l}\text { When participants talk } \\
\text { about anything that they } \\
\text { would change/add about } \\
\text { the topics covered }\end{array}$ & $\begin{array}{l}\text { When participants talk } \\
\text { about the need for } \\
\text { speakers to discuss } \\
\text { specific topics, } \\
\text { navigating the system. } \\
\text { grief }\end{array}$ & $\begin{array}{l}\text { When participants do } \\
\text { not mention anything } \\
\text { about speakers }\end{array}$ & $\begin{array}{l}\text { "Maybe each speaker } \\
\text { having specific topics in } \\
\text { order to create focused } \\
\text { conversations" } \\
\text { "How to navigate health } \\
\text { systems, resources at } \\
\text { Ryerson" } \\
\text { "Grief" }\end{array}$ \\
\hline $\begin{array}{l}\text { Culturally Specific } \\
\text { Topics }\end{array}$ & $\begin{array}{l}\text { When participants talk } \\
\text { about wanting } \\
\text { perspectives from } \\
\text { different Nations, and } \\
\text { ways to close the }\end{array}$ & $\begin{array}{l}\text { When different Nations } \\
\text { perspectives are } \\
\text { mentioned }\end{array}$ & $\begin{array}{l}\text { When participants talk } \\
\text { about mental health } \\
\text { content that they wanted } \\
\text { to learn about }\end{array}$ & $\begin{array}{l}\text { "Other tribal } \\
\text { beliefs/values" }\end{array}$ \\
\hline Cultural Closing & $\begin{array}{l}\text { When participants talk } \\
\text { about wanting to close } \\
\text { the circle in a particular } \\
\text { way }\end{array}$ & $\begin{array}{l}\text { Key words: Closing, } \\
\text { again }\end{array}$ & $\begin{array}{l}\text { When participants do } \\
\text { not mention anything } \\
\text { about closing the circle }\end{array}$ & $\begin{array}{l}\text { "Planning for the } \\
\text { immediate future } \\
\text { (closing the circle by } \\
\text { doing this)" } \\
\text { "Smudge again" }\end{array}$ \\
\hline No/No answer & $\begin{array}{l}\text { When participants do } \\
\text { not answer a question, or } \\
\text { did not want to learn } \\
\text { anything different }\end{array}$ & $\begin{array}{l}\text { Keywords: No, N/A, } \\
\text { nothing }\end{array}$ & $\begin{array}{l}\text { If participants mention } \\
\text { anything that they would } \\
\text { like to have learned }\end{array}$ & "No" "Nothing" \\
\hline \multicolumn{5}{|c|}{ Were the questions asked of you before and after acceptable to you? } \\
\hline Acceptable & $\begin{array}{l}\text { When participants state } \\
\text { that the questions were } \\
\text { acceptable and no }\end{array}$ & $\begin{array}{l}\text { Keywords: Yes, no } \\
\text { changes, acceptable }\end{array}$ & $\begin{array}{l}\text { If participants talk about } \\
\text { wanting more interactive } \\
\text { elements or dialogue }\end{array}$ & $\begin{array}{l}\text { "Acceptable. Don't } \\
\text { change anything" }\end{array}$ \\
\hline
\end{tabular}




\begin{tabular}{|l|l|l|l|l|}
\hline & changes are needed & $\begin{array}{l}\text { that is more open to } \\
\text { interpretation, then code } \\
\text { this under the } \\
\text { Participation/ } \\
\text { Engagement code }\end{array}$ & \\
\hline Miscellaneous & $\begin{array}{l}\text { Anything that doesn't fit } \\
\text { into any of the codes } \\
\text { listed above }\end{array}$ & & \\
\hline
\end{tabular}

Guidelines to keep in mind when coding:

(1) You don't have to code everything

(2) Be sure to include the Is, P1s, P2s, etc when you code

(3) Keep track of questions or uncertainties when you code so that we can clarify them when we chat next

(4) Try not to code identifying information

\section{Knowledge Translation Activity Follow-up Interview Codebook}

\begin{tabular}{|l|l|l|l|l|}
\hline \multicolumn{2}{|c|}{ Code Name } & \multicolumn{1}{|c|}{ Definition } & When to use Code & Examples \\
\hline Was there anything that you learned? (Merged Q1, Q2, Q3, Q4) & $\begin{array}{l}\text { When participants talk } \\
\text { about cultural coping } \\
\text { strategies that they } \\
\text { learned from the activity }\end{array}$ & $\begin{array}{l}\text { Keywords: cultural, } \\
\text { balance, wholistic, } \\
\text { physical, spiritual, } \\
\text { mental, emotional, } \\
\text { smudging }\end{array}$ & $\begin{array}{l}\text { When participants talk } \\
\text { about a coping strategy } \\
\text { that he/she learned from } \\
\text { the activity that is NOT } \\
\text { specific to Aboriginal } \\
\text { culture (something that } \\
\text { could be seen as a } \\
\text { and to know that } \\
\text { just being nervous about } \\
\text { something that I don't } \\
\text { need to be nervous } \\
\text { about. It's good to know } \\
\text { that one, it is a problem }\end{array}$ \\
\hline
\end{tabular}




\begin{tabular}{|c|c|c|c|c|}
\hline & & & strategy) & $\begin{array}{l}\text { and if you don't deal } \\
\text { with it is going to be a } \\
\text { serious problem and you } \\
\text { get burnt out" }\end{array}$ \\
\hline General Coping & $\begin{array}{l}\text { When participants talk } \\
\text { about coping strategies } \\
\text { they learned from the } \\
\text { activity that could be } \\
\text { seen as a mainstream } \\
\text { strategy (i.e., breathing, } \\
\text { nutrition) }\end{array}$ & $\begin{array}{l}\text { When participants talk } \\
\text { about general coping } \\
\text { strategies that they } \\
\text { learned from the activity }\end{array}$ & $\begin{array}{l}\text { When participants talk } \\
\text { about culturally specific } \\
\text { coping strategies that } \\
\text { they learned from the } \\
\text { activity }\end{array}$ & $\begin{array}{l}\text { "I learned about the } \\
\text { breathing, good } \\
\text { nutrition, exercise..." } \\
\text { "I guess I learned about } \\
\text { the way people cope } \\
\text { with their own unique } \\
\text { problems. I think it's } \\
\text { valuable to see how } \\
\text { people deal with } \\
\text { problems and where } \\
\text { they're coming from" }\end{array}$ \\
\hline Reinforced Knowledge & $\begin{array}{l}\text { When participants talk } \\
\text { about already having } \\
\text { mental health knowledge } \\
\text { and that the activity } \\
\text { reinforced what they } \\
\text { already knew }\end{array}$ & $\begin{array}{l}\text { When participants talk } \\
\text { about having mental } \\
\text { health knowledge and } \\
\text { that the activity gave the } \\
\text { same information that } \\
\text { they already knew }\end{array}$ & $\begin{array}{l}\text { When participants } d o \\
\text { not talk about the } \\
\text { activity covering a lot of } \\
\text { information that they } \\
\text { already knew }\end{array}$ & $\begin{array}{l}\text { "...I would like to say } \\
\text { that some things were } \\
\text { reinforced" }\end{array}$ \\
\hline Resources & $\begin{array}{l}\text { When participants talk } \\
\text { about gaining any } \\
\text { resources from the } \\
\text { booklet or presentations } \\
\text { that were provided }\end{array}$ & $\begin{array}{l}\text { Keywords: Resource, } \\
\text { resource book, resource } \\
\text { booklet }\end{array}$ & $\begin{array}{l}\text { When participants do } \\
\text { not mention resources } \\
\text { for mental health that } \\
\text { they learned from the } \\
\text { activity }\end{array}$ & $\begin{array}{l}\text { "Liked hearing about the } \\
\text { resources and agencies" } \\
\text { "I think the resource }\end{array}$ \\
\hline
\end{tabular}




\begin{tabular}{|c|c|c|c|c|}
\hline & during the activity & & & $\begin{array}{l}\text { book you made was } \\
\text { really great" } \\
\text { "To have a resource } \\
\text { where you can see } \\
\text { everything and you have } \\
\text { an idea how the system } \\
\text { works it's a lot easier for } \\
\text { people who are problem } \\
\text { solvers." }\end{array}$ \\
\hline No/Can't Remember & $\begin{array}{l}\text { When participants state } \\
\text { that they can't remember } \\
\text { or that it is difficult to } \\
\text { remember anything they } \\
\text { learned }\end{array}$ & $\begin{array}{l}\text { Use this code even if the } \\
\text { participant does think of } \\
\text { something, but begins by } \\
\text { stating that it is hard to } \\
\text { remember or they can't } \\
\text { remember }\end{array}$ & $\begin{array}{l}\text { Do not use code when a } \\
\text { participant does not talk } \\
\text { at all about not } \\
\text { remembering the activity }\end{array}$ & "It is hard to remember" \\
\hline \multicolumn{5}{|c|}{ How could the activity be changed to be helpful to you? } \\
\hline Acceptable & $\begin{array}{l}\text { When participants talk } \\
\text { about the ways that the } \\
\text { activity worked well for } \\
\text { them during the activity }\end{array}$ & Keywords: good, liked & $\begin{array}{l}\text { Do not use code when } \\
\text { they suggest something } \\
\text { to change }\end{array}$ & $\begin{array}{l}\text { "I thought it was pretty } \\
\text { good the way it was" } \\
\text { "I think the size...I } \\
\text { know you sometimes } \\
\text { plan for a larger group, } \\
\text { but I thought the smaller } \\
\text { group was really good" }\end{array}$ \\
\hline Recommendations & $\begin{array}{l}\text { When participants talk } \\
\text { about how the activity }\end{array}$ & $\begin{array}{l}\text { Any recommendation or } \\
\text { suggestion }\end{array}$ & $\begin{array}{l}\text { Do not use code when } \\
\text { participants are talking }\end{array}$ & $\begin{array}{l}\text { "I can't remember if we } \\
\text { closed it. I remember }\end{array}$ \\
\hline
\end{tabular}




\begin{tabular}{|c|c|c|c|c|}
\hline & $\begin{array}{l}\text { could be changed such } \\
\text { as time, length, method, } \\
\text { content, things to change } \\
\text { in the resource book, } \\
\text { speakers, adding } \\
\text { flipcharts, have more of } \\
\text { a focus be on coping }\end{array}$ & & $\begin{array}{l}\text { about things that were } \\
\text { helpful to them or that } \\
\text { they liked, as this would } \\
\text { not be a } \\
\text { recommendation }\end{array}$ & $\begin{array}{l}\text { having a feeling that we } \\
\text { just dispersed" } \\
\text { I can't remember if in } \\
\text { "the call out for } \\
\text { participants...I } \\
\text { remember thinking that } \\
\text { the two people who were } \\
\text { invited... I was like, oh I } \\
\text { didn't expect these } \\
\text { people" }\end{array}$ \\
\hline \multicolumn{5}{|c|}{ Have you/do you plan to used anything you learned in your daily life? (Merged 8 \& 10) } \\
\hline Using General Coping & $\begin{array}{l}\text { When participants talk } \\
\text { about general coping } \\
\text { strategies they have used } \\
\text { or plan to use from the } \\
\text { activity in their daily } \\
\text { lives }\end{array}$ & $\begin{array}{l}\text { General coping } \\
\text { strategies such as } \\
\text { breathing and positive } \\
\text { self talk } \\
\text { Please include how often } \\
\text { they use this strategy } \\
\text { under this code if this } \\
\text { information is available }\end{array}$ & $\begin{array}{l}\text { When participants talk } \\
\text { about culturally specific } \\
\text { coping strategies }\end{array}$ & $\begin{array}{l}\text { "Breathing...A few } \\
\text { times a day, especially } \\
\text { now because it's } \\
\text { midterms and final } \\
\text { papers." }\end{array}$ \\
\hline Using Cultural Coping & $\begin{array}{l}\text { When participants talk } \\
\text { about culturally specific } \\
\text { coping strategies that } \\
\text { they implemented since } \\
\text { the activity or plan to } \\
\text { implement }\end{array}$ & $\begin{array}{l}\text { Culturally specific } \\
\text { coping strategies such as } \\
\text { smudging more, } \\
\text { incorporating more } \\
\text { balance }\end{array}$ & $\begin{array}{l}\text { Do not use this code if } \\
\text { participants talk about } \\
\text { general coping strategies } \\
\text { used in mainstream } \\
\text { contexts }\end{array}$ & $\begin{array}{l}\text { "Once in a while I } \\
\text { smudge" }\end{array}$ \\
\hline
\end{tabular}




\begin{tabular}{|c|c|c|c|c|}
\hline & & $\begin{array}{l}\text { Please include how often } \\
\text { they use this strategy } \\
\text { under this code if this } \\
\text { information is available }\end{array}$ & $\begin{array}{l}\text { Do not use this code if } \\
\text { participants state that } \\
\text { they have not or do not } \\
\text { plan to use anything they } \\
\text { learned in their daily life }\end{array}$ & \\
\hline No/Can't remember & $\begin{array}{l}\text { When participants state } \\
\text { that they can't } \\
\text { remember, have not or } \\
\text { do not plan to use } \\
\text { anything from the } \\
\text { activity in their daily } \\
\text { lives }\end{array}$ & $\begin{array}{l}\text { Include under this code } \\
\text { the reason that they may } \\
\text { state for not using } \\
\text { anything in their daily } \\
\text { life, if this is available }\end{array}$ & $\begin{array}{l}\text { Do not use code if } \\
\text { participants give one or } \\
\text { more examples of things } \\
\text { they may use in their } \\
\text { daily lives from the } \\
\text { activity }\end{array}$ & $\begin{array}{l}\text { "No" } \\
\text { "I missed the coping } \\
\text { portion of the activity } \\
\text { and am having a hard } \\
\text { time remembering" }\end{array}$ \\
\hline \multicolumn{5}{|c|}{ What is the biggest take home message? } \\
\hline Cultural Take Home & $\begin{array}{l}\text { When participants talk } \\
\text { about culturally specific } \\
\text { messages from the } \\
\text { activity }\end{array}$ & $\begin{array}{l}\text { Keywords: community, } \\
\text { wholistic, resilient, } \\
\text { strength }\end{array}$ & $\begin{array}{l}\text { When participants talk } \\
\text { about messages that can } \\
\text { be applied at an } \\
\text { individual level rather } \\
\text { than at a community } \\
\text { level }\end{array}$ & $\begin{array}{l}\text { "...we are coming from } \\
\text { worldview that doesn't } \\
\text { see the stigma and that } \\
\text { there is a focus on } \\
\text { wholistic healing" } \\
\text { "remember where you } \\
\text { come from, remember } \\
\text { that you are part of a } \\
\text { community who is } \\
\text { strong and resilient and } \\
\text { that we all have inherent } \\
\text { strengths" }\end{array}$ \\
\hline Individual Take Home & $\begin{array}{l}\text { When participants talk } \\
\text { about messages they }\end{array}$ & $\begin{array}{l}\text { When participants do } \\
\text { not mention cultural or }\end{array}$ & $\begin{array}{l}\text { When participants talk } \\
\text { about culturally specific }\end{array}$ & $\begin{array}{l}\text { "I guess just that... you } \\
\text { have to deal with your }\end{array}$ \\
\hline
\end{tabular}




\begin{tabular}{|c|c|c|c|c|}
\hline & $\begin{array}{l}\text { gained from this project } \\
\text { that can be applied at an } \\
\text { individual level }\end{array}$ & $\begin{array}{l}\text { community specific } \\
\text { messages that they } \\
\text { gained from this project }\end{array}$ & $\begin{array}{l}\text { messages from the } \\
\text { activity rather than } \\
\text { individual level } \\
\text { messages }\end{array}$ & $\begin{array}{l}\text { problems, pretty much, } \\
\text { and there's ways to deal } \\
\text { with it and it's not } \\
\text { always just one thing" }\end{array}$ \\
\hline \multicolumn{5}{|c|}{ What is the current state of student mental health knowledge? } \\
\hline Aboriginal vs. Western & $\begin{array}{l}\text { When participants talk } \\
\text { about the struggle } \\
\text { between Aboriginal } \\
\text { worldview in a western- } \\
\text { dominated society }\end{array}$ & $\begin{array}{l}\text { When Aboriginal and } \\
\text { Western ideologies are } \\
\text { compared and contrasted }\end{array}$ & $\begin{array}{l}\text { When Aboriginal and } \\
\text { Western ideologies are } \\
\text { not discussed }\end{array}$ & $\begin{array}{l}\text { "I think Aboriginals } \\
\text { understand their own } \\
\text { wellbeing when they are } \\
\text { amongst themselves. But } \\
\text { when they go out into } \\
\text { the white man's world } \\
\text { it's different. It's kind of } \\
\text { hard to get along with." }\end{array}$ \\
\hline MH Stigma & $\begin{array}{l}\text { When participants talk } \\
\text { about the stigma } \\
\text { associated with mental } \\
\text { health concerns }\end{array}$ & $\begin{array}{l}\text { Keywords: stigma, } \\
\text { defensive }\end{array}$ & $\begin{array}{l}\text { When participants do } \\
\text { not discuss stigma or } \\
\text { any issues with labels }\end{array}$ & $\begin{array}{l}\text { "...there is still a lot of } \\
\text { stigma around getting } \\
\text { help" }\end{array}$ \\
\hline Improvements Needed & $\begin{array}{l}\text { When participants talk } \\
\text { about improvements that } \\
\text { need to be made in the } \\
\text { context of discussing the } \\
\text { current state of mental } \\
\text { health knowledge }\end{array}$ & $\begin{array}{l}\text { When participant talks } \\
\text { about ways that MH } \\
\text { knowledge can be } \\
\text { improved }\end{array}$ & $\begin{array}{l}\text { When participants do } \\
\text { not discuss any } \\
\text { improvements to our } \\
\text { perspectives on mental } \\
\text { health knowledge } \\
\text { resources or coping }\end{array}$ & $\begin{array}{l}\text { "...I think there is still } \\
\text { lots of work to be done. } \\
\text { Not just around our } \\
\text { Aboriginal identities but } \\
\text { also other intersecting } \\
\text { identities." }\end{array}$ \\
\hline \multicolumn{5}{|c|}{ What does the future hold for student mental health knowledge? } \\
\hline $\begin{array}{l}\text { Resources readily } \\
\text { available }\end{array}$ & $\begin{array}{l}\text { When participants talk } \\
\text { about the need for } \\
\text { resources to be readily } \\
\text { available for all people } \\
\text { and for youth in }\end{array}$ & $\begin{array}{l}\text { Use this code when } \\
\text { someone specifically } \\
\text { talks about targeting } \\
\text { youth in the future as } \\
\text { such resources will need }\end{array}$ & $\begin{array}{l}\text { Do not use this code if } \\
\text { they are answering a } \\
\text { different question }\end{array}$ & $\begin{array}{l}\text { "The younger } \\
\text { generations are more } \\
\text { open and they want to } \\
\text { change. It crosses over } \\
\text { between the age groups }\end{array}$ \\
\hline
\end{tabular}




\begin{tabular}{|c|c|c|c|c|}
\hline & particular & $\begin{array}{l}\text { to be readily available } \\
\text { for them so they can } \\
\text { move their communities } \\
\text { forward }\end{array}$ & & $\begin{array}{l}\text { so that older people now } \\
\text { are more understanding, } \\
\text { more respectful and } \\
\text { they're more willing. So, } \\
\text { I think it's really great to } \\
\text { focus on young people } \\
\text { who are willing to try } \\
\text { new things and share } \\
\text { that and be an example" }\end{array}$ \\
\hline Cultural Revitalization & $\begin{array}{l}\text { When participants talk } \\
\text { about bringing their } \\
\text { culture back through } \\
\text { learning about their } \\
\text { history, bringing } \\
\text { communities together, or } \\
\text { decolonization }\end{array}$ & $\begin{array}{l}\text { Use this code if they } \\
\text { allude to cultural } \\
\text { revitalization or if they } \\
\text { specifically talk about } \\
\text { cultural revitalization }\end{array}$ & $\begin{array}{l}\text { Do not use this code if } \\
\text { they are answering a } \\
\text { different question }\end{array}$ & $\begin{array}{l}\text { "I think social media has } \\
\text { been really awesome at } \\
\text { promoting } \\
\text { decolonization theory, } \\
\text { for example" } \\
\text { "Just that we should be } \\
\text { learning our own history } \\
\text { instead of listening to } \\
\text { the White Mans' history. } \\
\text { That's all we hear, how } \\
\text { they did this and that but } \\
\text { they never say anything } \\
\text { about what the natives } \\
\text { did" }\end{array}$ \\
\hline Education & $\begin{array}{l}\text { When participants talk } \\
\text { about the importance of } \\
\text { educating the Aboriginal } \\
\text { population and having } \\
\text { Aboriginal authors and } \\
\text { perspectives in schools }\end{array}$ & $\begin{array}{l}\text { Keywords: degrees, } \\
\text { education, authors, } \\
\text { schools }\end{array}$ & $\begin{array}{l}\text { Do not use this code if } \\
\text { they are answering a } \\
\text { different question }\end{array}$ & $\begin{array}{l}\text { "I think that they've got } \\
\text { to start writing their own } \\
\text { books I guess. There } \\
\text { aren't too many people } \\
\text { who write their own } \\
\text { books, like education. }\end{array}$ \\
\hline
\end{tabular}




\begin{tabular}{|l|l|l|l|l|}
\hline & & & & $\begin{array}{l}\text { They have to get } \\
\text { educated" }\end{array}$ \\
\hline
\end{tabular}

Guidelines to keep in mind when coding:

(1) You don't have to code everything

(2) Be sure to include the Is, P1s, P2s, etc when you code

(3) Keep track of questions or uncertainties when you code so that we can clarify them when we chat next

(4) Try not to code identifying information 
Final Follow-Up/Debriefing Phase Codebook

\begin{tabular}{|c|c|c|c|c|}
\hline Code Name & Definition & When to use Code & When not to use code & Examples \\
\hline \multicolumn{5}{|c|}{ Have you heard anything/what do you think about how the activity went? (merged a \& b) } \\
\hline $\begin{array}{l}\text { Activity: Sense of } \\
\text { Community }\end{array}$ & $\begin{array}{l}\text { When participants talk } \\
\text { about the fact that the } \\
\text { activity brought the } \\
\text { community together and } \\
\text { that it was important for } \\
\text { it to be done by a student } \\
\text { under this question }\end{array}$ & $\begin{array}{l}\text { Keywords: community, } \\
\text { student initiated }\end{array}$ & $\begin{array}{l}\text { Do not use this code } \\
\text { when participants are } \\
\text { talking about their take } \\
\text { home message }\end{array}$ & $\begin{array}{l}\text { "They appreciated that it } \\
\text { was one of their } \\
\text { colleagues; one of their } \\
\text { own peers was the one } \\
\text { doing a study like this. } \\
\text { Your being able to speak } \\
\text { to them, I think that } \\
\text { made a difference that it } \\
\text { was coming from one of } \\
\text { their own people within } \\
\text { the circle and not } \\
\text { someone that was } \\
\text { already accomplished. I } \\
\text { think that sense of } \\
\text { respect brought the } \\
\text { knowledge level up" }\end{array}$ \\
\hline $\begin{array}{l}\text { Activity: } \\
\text { Growth/Learning }\end{array}$ & $\begin{array}{l}\text { When participants talk } \\
\text { about how much/what } \\
\text { they learned, how } \\
\text { much/what students } \\
\text { learned, or how } \\
\text { much/what the } \\
\text { researchers have learned }\end{array}$ & $\begin{array}{l}\text { Use this code when } \\
\text { talking about } \\
\text { normalizing mental } \\
\text { health as well } \\
\text { Use code when talking }\end{array}$ & $\begin{array}{l}\text { Do not use this code } \\
\text { when not speaking } \\
\text { specifically about the } \\
\text { activity }\end{array}$ & $\begin{array}{l}\text { "Working with you she } \\
\text { said she learned a lot and } \\
\text { I think that transfers to } \\
\text { the students. Even if you } \\
\text { just had the core, it } \\
\text { resonated with the } \\
\text { students, because I think } \\
\text { you had people coming }\end{array}$ \\
\hline
\end{tabular}




\begin{tabular}{|c|c|c|c|c|}
\hline & & $\begin{array}{l}\text { about providing students } \\
\text { with language to } \\
\text { validate/cope their } \\
\text { mental health experience }\end{array}$ & & $\begin{array}{l}\text { from all different } \\
\text { directions. I think it did. } \\
\text { I think it's really } \\
\text { imperative that students } \\
\text { look at mental health as } \\
\text { not just being the status } \\
\text { quo or the typical view } \\
\text { of mental health. It } \\
\text { affects everybody one } \\
\text { way or the other, } \\
\text { especially students } \\
\text { because there are outside } \\
\text { pressures" }\end{array}$ \\
\hline $\begin{array}{l}\text { Activity: Reflections/ } \\
\text { Recommendations }\end{array}$ & $\begin{array}{l}\text { When participants talk } \\
\text { about how they may } \\
\text { have used the activity in } \\
\text { a different way in } \\
\text { hindsight and what they } \\
\text { liked/would change } \\
\text { about various portions } \\
\text { the project }\end{array}$ & $\begin{array}{l}\text { Keywords: hindsight, } \\
\text { flexibility, change }\end{array}$ & $\begin{array}{l}\text { Do not use when } \\
\text { participants don't } \\
\text { mention anything they } \\
\text { would have done } \\
\text { differently in utilizing } \\
\text { the activity that was } \\
\text { implemented or offer } \\
\text { any general reflections } \\
\text { Do not use when they } \\
\text { are not discussing the } \\
\text { activity }\end{array}$ & $\begin{array}{l}\text { "In hindsight I think it } \\
\text { would have been helpful } \\
\text { for another of the staff to } \\
\text { attend just so we might } \\
\text { look at how we could } \\
\text { build that into our } \\
\text { programming, how we } \\
\text { could engage or partner } \\
\text { with our own health } \\
\text { center to get them re- } \\
\text { thinking well-being, } \\
\text { balance, et cetera" }\end{array}$ \\
\hline Activity: Unsure & $\begin{array}{l}\text { When participants were } \\
\text { unsure of the knowledge } \\
\text { or perceptions of } \\
\text { Aboriginal students that } \\
\text { stemmed from the }\end{array}$ & $\begin{array}{l}\text { Keywords: I don't know, } \\
\text { unsure, }\end{array}$ & $\begin{array}{l}\text { Do not use when they } \\
\text { are not discussing the } \\
\text { activity }\end{array}$ & $\begin{array}{l}\text { "I don't know if I can } \\
\text { speak specifically on } \\
\text { that" }\end{array}$ \\
\hline
\end{tabular}




\begin{tabular}{|c|c|c|c|c|}
\hline & activity & & & \\
\hline \multicolumn{5}{|c|}{ How do you see your role in this project? } \\
\hline Role: General Support & $\begin{array}{l}\text { When participants talk } \\
\text { their role as supporting } \\
\text { the project in various } \\
\text { ways such as helping to } \\
\text { recruit }\end{array}$ & $\begin{array}{l}\text { Keywords: liaison, } \\
\text { support, advocate, } \\
\text { connect } \\
\text { Include under this code } \\
\text { anything that } \\
\text { participants say about } \\
\text { believing in this project } \\
\text { or being excited about } \\
\text { this project }\end{array}$ & $\begin{array}{l}\text { Do not use this code } \\
\text { when participants are } \\
\text { not discussing their role } \\
\text { in the overall project } \\
\text { Do not use this code if } \\
\text { participants are talking } \\
\text { about supporting the } \\
\text { activity or the research } \\
\text { in a cultural way/context }\end{array}$ & $\begin{array}{l}\text { "I'm just one of those } \\
\text { connectors. I was one of } \\
\text { those people who was } \\
\text { able to be suggesting, } \\
\text { sharing opinions and be } \\
\text { encouraging, and just be } \\
\text { able to stress the } \\
\text { importance of what you } \\
\text { are doing and the } \\
\text { outcomes that could } \\
\text { potentially happen" }\end{array}$ \\
\hline Role: Cultural Supports & $\begin{array}{l}\text { When participants talk } \\
\text { about offering cultural } \\
\text { support to the activity or } \\
\text { the researcher }\end{array}$ & $\begin{array}{l}\text { Use this code when } \\
\text { participants talk about } \\
\text { helping to build } \\
\text { relationships as is } \\
\text { important in an } \\
\text { Aboriginal context and } \\
\text { when talking about } \\
\text { believing in the project } \\
\text { in an Aboriginal context } \\
\text { (i.e., using ceremony) } \\
\text { Use this quote when } \\
\text { participants talk about } \\
\text { contributing an } \\
\text { Aboriginal perspective }\end{array}$ & $\begin{array}{l}\text { Do not use this code } \\
\text { when participants are } \\
\text { not discussing their role } \\
\text { in the overall project } \\
\text { Do not use this code if } \\
\text { participants are talking } \\
\text { about supporting the } \\
\text { activity or the research } \\
\text { in a general context or a } \\
\text { context that could be } \\
\text { perceived as mainstream }\end{array}$ & $\begin{array}{l}\text { "Every time I did a pipe } \\
\text { ceremony, I thought } \\
\text { about you and thought, } \\
\text { "well, I hope she is } \\
\text { carrying on because this } \\
\text { is important"". }\end{array}$ \\
\hline
\end{tabular}




\begin{tabular}{|c|c|c|c|c|}
\hline & & in support of the project & & \\
\hline Role: Unclear & $\begin{array}{l}\text { When participants talk } \\
\text { about being unclear } \\
\text { regarding their role, } \\
\text { what the project was } \\
\text { about, or having a lack } \\
\text { of communication }\end{array}$ & $\begin{array}{l}\text { Keywords: } \\
\text { Communicate, } \\
\text { difficulties, wasn't sure }\end{array}$ & $\begin{array}{l}\text { Do not use this code } \\
\text { when participants are } \\
\text { not discussing their role } \\
\text { in the overall project }\end{array}$ & $\begin{array}{l}\text { "I think in the beginning, } \\
\text { because of the transition } \\
\text { of [name] not being } \\
\text { here, I think a lot of it } \\
\text { was difficult for me to } \\
\text { pick up because I felt a } \\
\text { little bit behind. There } \\
\text { was an intention of } \\
\text { having more } \\
\text { conversations after we } \\
\text { had that first } \\
\text { conversation with } \\
\text { [name] and I and then } \\
\text { [name] was just gone. I } \\
\text { was like, "wait"!" }\end{array}$ \\
\hline Role: Participant & $\begin{array}{l}\text { When participants talk } \\
\text { about their role as being } \\
\text { a participant in the } \\
\text { project }\end{array}$ & $\begin{array}{l}\text { Use this code if the } \\
\text { participant talks about } \\
\text { specific information that } \\
\text { they may have } \\
\text { contributed to a circle in } \\
\text { which they may have } \\
\text { participated }\end{array}$ & $\begin{array}{l}\text { Do not use this code } \\
\text { when participants are } \\
\text { not discussing their role } \\
\text { in the overall project }\end{array}$ & $\begin{array}{l}\text { "I had done individual } \\
\text { counselling, I had gone } \\
\text { to a group that focused } \\
\text { on anger and release but } \\
\text { there were shortfalls of } \\
\text { both. Therapy and } \\
\text { counselling fell short } \\
\text { because the model was } \\
\text { so rigid. There was very } \\
\text { little place of recognition } \\
\text { or acknowledgement of } \\
\text { being an Aboriginal } \\
\text { person and bringing that } \\
\text { intergenerational piece } \\
\text { that I'm sure I talked } \\
\text { about, the things we }\end{array}$ \\
\hline
\end{tabular}




\begin{tabular}{|c|c|c|c|c|}
\hline & & & & $\begin{array}{l}\text { inherit from the people } \\
\text { we come from, and the } \\
\text { intergenerational trauma } \\
\text { and the impact" }\end{array}$ \\
\hline \multicolumn{5}{|c|}{ What are the key take away messages from this project? } \\
\hline Take Away: Community & $\begin{array}{l}\text { When participants talk } \\
\text { about how their take } \\
\text { away message was how } \\
\text { to incorporate graduate } \\
\text { work into our } \\
\text { community or anything } \\
\text { to do with student's } \\
\text { working on the project } \\
\text { and engaging the } \\
\text { community }\end{array}$ & & $\begin{array}{l}\text { Do not use this code if } \\
\text { they are answering the } \\
\text { question about the } \\
\text { activity }\end{array}$ & $\begin{array}{l}\text { "...I think it was a really } \\
\text { good example of how } \\
\text { we can incorporate } \\
\text { graduate work into our } \\
\text { community and actually } \\
\text { have it be a community } \\
\text { thing. I think even some } \\
\text { of our students got to } \\
\text { know each other through } \\
\text { your activities so that } \\
\text { was nice..." } \\
\\
\text { "Just seeing some of the } \\
\text { students relate to it and } \\
\text { embracing it..." }\end{array}$ \\
\hline $\begin{array}{l}\text { Take Away: Cultural } \\
\text { Revitalization }\end{array}$ & $\begin{array}{l}\text { When participants talk } \\
\text { about this project as } \\
\text { decolonizing and the } \\
\text { importance of having } \\
\text { such knowledge come } \\
\text { from community } \\
\text { members }\end{array}$ & $\begin{array}{l}\text { Use this code when } \\
\text { participants are } \\
\text { specifically talking } \\
\text { about the cultural } \\
\text { knowledge that needs to } \\
\text { be communicated }\end{array}$ & $\begin{array}{l}\text { Do not use this code if } \\
\text { answering a different } \\
\text { question }\end{array}$ & $\begin{array}{l}\text { "That knowledge } \\
\text { production needs to be } \\
\text { back in the hands of the } \\
\text { people who need it. It's } \\
\text { not beneficial if it's just } \\
\text { academic. It has to come } \\
\text { from them, it has to } \\
\text { return from them, and } \\
\text { then it has to come from } \\
\text { them again, and return to }\end{array}$ \\
\hline
\end{tabular}




\begin{tabular}{|c|c|c|c|c|}
\hline & & & & them again" \\
\hline $\begin{array}{l}\text { Take Away: MH } \\
\text { Knowledge/Information }\end{array}$ & $\begin{array}{l}\text { When participants talk } \\
\text { about the need to } \\
\text { increase mental health } \\
\text { knowledge to combat } \\
\text { stigma or the task of } \\
\text { narrowing such } \\
\text { knowledge down to be } \\
\text { managed }\end{array}$ & $\begin{array}{l}\text { Keywords: Information, } \\
\text { knowledge }\end{array}$ & $\begin{array}{l}\text { Do not use this code if } \\
\text { answering a different } \\
\text { question }\end{array}$ & $\begin{array}{l}\text { "...the average person } \\
\text { needs to know more } \\
\text { about mental health } \\
\text { issues to remove it from } \\
\text { the stereotypical } \\
\text { perceptions of mental } \\
\text { health and moving in } \\
\text { toward a more } \\
\text { normalizing event that } \\
\text { can happen to anyone" }\end{array}$ \\
\hline \multicolumn{5}{|c|}{ Where are we with the current state of MH knowledge? Where are we going? } \\
\hline Present/Future: Stigma & $\begin{array}{l}\text { When participants talk } \\
\text { about students being } \\
\text { afraid to talk about } \\
\text { mental health or stigma } \\
\text { being not as much of an } \\
\text { issue as it used to be or } \\
\text { anything to do with } \\
\text { stigma in the future }\end{array}$ & $\begin{array}{l}\text { Keywords: afraid, } \\
\text { stigma }\end{array}$ & $\begin{array}{l}\text { Do not use if they are } \\
\text { answering a different } \\
\text { question }\end{array}$ & $\begin{array}{l}\text { "I think that students are } \\
\text { still afraid, even though } \\
\text { so many of them have } \\
\text { people in their families } \\
\text { and in their lives that } \\
\text { really struggle" }\end{array}$ \\
\hline $\begin{array}{l}\text { Present/Future: } \\
\text { Resources }\end{array}$ & $\begin{array}{l}\text { When participants talk } \\
\text { about the current and } \\
\text { future state of resources } \\
\text { right now, including } \\
\text { opening doors for } \\
\text { resources } \\
\text { When participants talk } \\
\text { about the need to have }\end{array}$ & Keywords: resources & $\begin{array}{l}\text { Do not use if they are } \\
\text { answering a different } \\
\text { question }\end{array}$ & $\begin{array}{l}\text { "I think a lot of the } \\
\text { resources for Aboriginal } \\
\text { students to access are } \\
\text { very addictions focused } \\
\text { and are focused on } \\
\text { things like homelessness } \\
\text { and unstable housing } \\
\text { and stuff. That may not } \\
\text { be students primary } \\
\text { concern or they may not }\end{array}$ \\
\hline
\end{tabular}




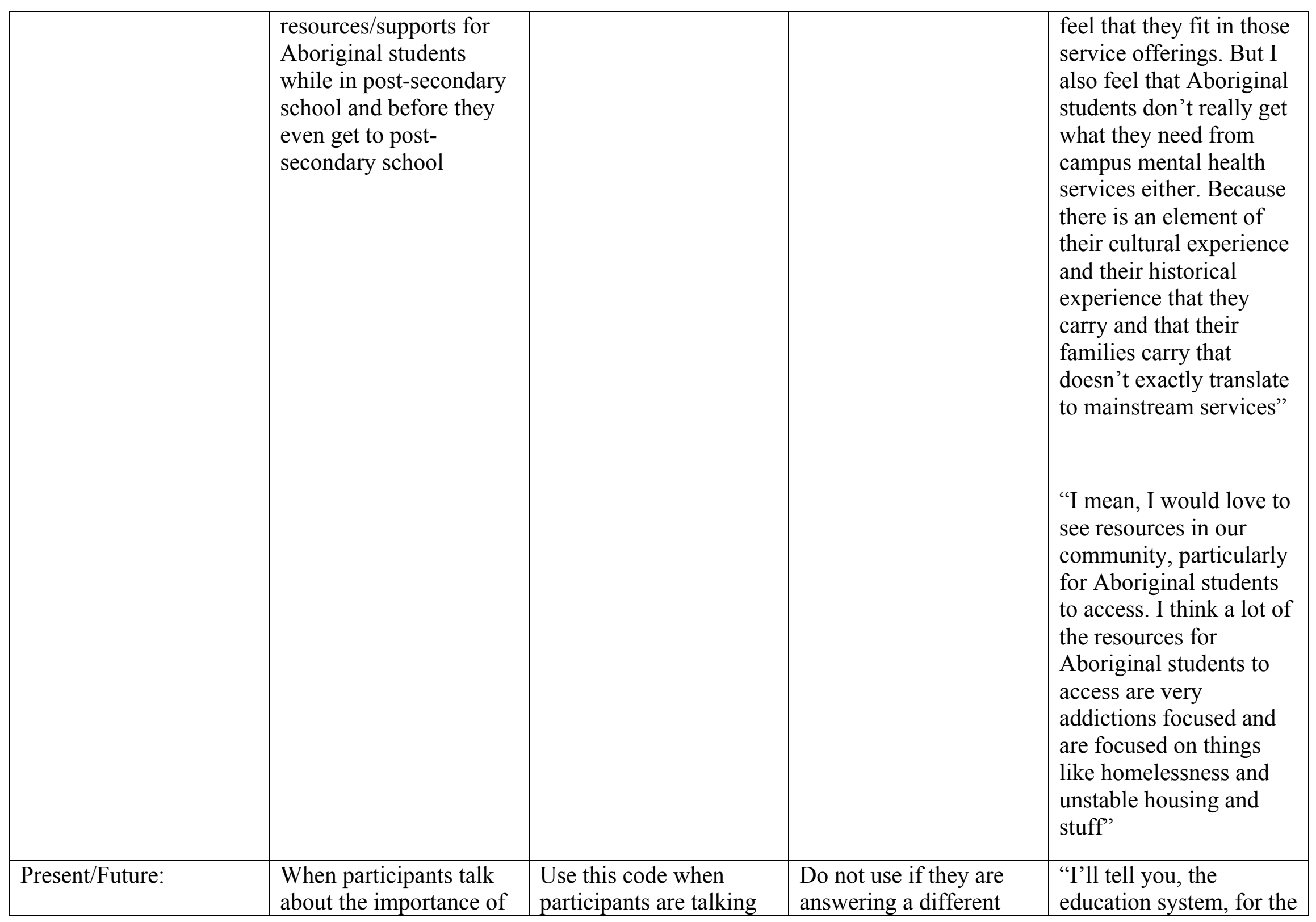




\begin{tabular}{|c|c|c|c|c|}
\hline Education & $\begin{array}{l}\text { acknowledging } \\
\text { Indigenous Peoples and } \\
\text { their perspectives in the } \\
\text { education system and } \\
\text { about the importance of } \\
\text { educating students about } \\
\text { mental health }\end{array}$ & $\begin{array}{l}\text { about what needs to } \\
\text { change in the future } \\
\text { about education or what } \\
\text { the current state of } \\
\text { education } \\
\text { Use this code when } \\
\text { participants talk about } \\
\text { the need to encourage } \\
\text { dreams of little ones } \\
\text { and about what needs to } \\
\text { change in the future }\end{array}$ & question & $\begin{array}{l}\text { longest time, did not } \\
\text { emphasize our existence } \\
\text { and in the proper context } \\
\text { and that does create } \\
\text { mental stress. From } \\
\text { there they graduated to } \\
\text { other issues like } \\
\text { negativity, additions" }\end{array}$ \\
\hline $\begin{array}{l}\text { Present/Future: } \mathrm{MH} \\
\text { Aboriginal Perspective }\end{array}$ & $\begin{array}{l}\text { When participants talk } \\
\text { about the need to } \\
\text { decolonize perspectives } \\
\text { on mental health and the } \\
\text { need to look at it from a } \\
\text { wholistic lens }\end{array}$ & $\begin{array}{l}\text { Keywords: Decolonize, } \\
\text { wholistic, continuum, } \\
\text { normalize } \\
\text { Use this code when } \\
\text { talking this theme in the } \\
\text { present tense } \\
\text { Use this code when } \\
\text { participants note } \\
\text { examples of instances } \\
\text { where they tried to } \\
\text { implement practices to } \\
\text { increase wholism, but } \\
\text { were unable to do so } \\
\text { because of how the }\end{array}$ & $\begin{array}{l}\text { Do not use if they are } \\
\text { answering a different } \\
\text { question }\end{array}$ & $\begin{array}{l}\text { "My hope is that as we } \\
\text { continue to de-colonize, } \\
\text { if I think back even five } \\
\text { to ten years, my own } \\
\text { understanding of being } \\
\text { Aboriginal was different } \\
\text { than it is today. As we } \\
\text { de-colonize as } \\
\text { communities and we get } \\
\text { healthier I hope that we } \\
\text { can return to an } \\
\text { understanding of mental } \\
\text { health being a } \\
\text { continuum that we all } \\
\text { fluctuate on, and that } \\
\text { having issues with } \\
\text { mental health is normal" }\end{array}$ \\
\hline
\end{tabular}




\begin{tabular}{|c|c|c|c|c|}
\hline & & $\begin{array}{l}\text { system currently } \\
\text { operates }\end{array}$ & & \\
\hline \multicolumn{5}{|c|}{ What impact would there be if I conducted follow-up interview or someone else conducted these interviews? } \\
\hline $\begin{array}{l}\text { Evaluate: Both } \\
\text { Pros/Cons }\end{array}$ & $\begin{array}{l}\text { When participants talk } \\
\text { about the pros and cons } \\
\text { of either evaluating your } \\
\text { own program or having } \\
\text { someone else do it }\end{array}$ & $\begin{array}{l}\text { Use this code when } \\
\text { participants are talking } \\
\text { about both bringing } \\
\text { someone else in or not }\end{array}$ & $\begin{array}{l}\text { Do not use if they are } \\
\text { answering a different } \\
\text { question }\end{array}$ & $\begin{array}{l}\text { "I think when you ask } \\
\text { the questions you run the } \\
\text { risk of two things. Either } \\
\text { people who like you } \\
\text { being really positive } \\
\text { rather than accurate, or } \\
\text { people who either didn't } \\
\text { like you or didn't like } \\
\text { the program being very } \\
\text { negative about it. So it is } \\
\text { a running risk. I think } \\
\text { with someone else } \\
\text { asking, it removes some } \\
\text { performance anxiety or } \\
\text { people-pleasing anxiety. } \\
\text { But I think either way it } \\
\text { can give accurate } \\
\text { information it may just } \\
\text { be that how it is put- } \\
\text { forth or how it is said } \\
\text { may be different..." }\end{array}$ \\
\hline $\begin{array}{l}\text { Evaluate: } \\
\text { Recommendations }\end{array}$ & $\begin{array}{l}\text { When participants talk } \\
\text { ways that the evaluation } \\
\text { could have been done } \\
\text { differently }\end{array}$ & $\begin{array}{l}\text { Example: bringing } \\
\text { someone else in to } \\
\text { conduct the interviews } \\
\text { with me }\end{array}$ & $\begin{array}{l}\text { Do not use if they are } \\
\text { answering a different } \\
\text { question }\end{array}$ & $\begin{array}{l}\text { "I think about some of } \\
\text { the classes that get } \\
\text { offered in community } \\
\text { settings. Often they will } \\
\text { have the instructor and a } \\
\text { support person" }\end{array}$ \\
\hline
\end{tabular}




\begin{tabular}{|c|c|c|c|c|}
\hline Evaluate: Relationships & $\begin{array}{l}\text { When participants talk } \\
\text { about the importance of } \\
\text { relationships when } \\
\text { working in an } \\
\text { Aboriginal context and } \\
\text { how this might make it a } \\
\text { better idea to evaluate } \\
\text { your own project }\end{array}$ & $\begin{array}{l}\text { Keywords: Relationship, } \\
\text { trust }\end{array}$ & $\begin{array}{l}\text { Do not use if they are } \\
\text { answering a different } \\
\text { question }\end{array}$ & $\begin{array}{l}\text { "Well, the most } \\
\text { important thing I think } \\
\text { you accomplished was } \\
\text { the trust factor. Gaining } \\
\text { that trust is the key to } \\
\text { having a person open up. } \\
\text { So, for you to follow-up } \\
\text { is showing sincerity and } \\
\text { the fact that people saw } \\
\text { you again showed that } \\
\text { this young lady really } \\
\text { believes in what she is } \\
\text { doing" }\end{array}$ \\
\hline \multicolumn{5}{|c|}{ Do you think this project addressed any community needs? } \\
\hline Needs: Community & $\begin{array}{l}\text { When participants talk } \\
\text { about bringing the } \\
\text { community together or } \\
\text { creating a space for } \\
\text { students to talk about } \\
\text { mental health }\end{array}$ & $\begin{array}{l}\text { Keywords: community, } \\
\text { safe space }\end{array}$ & $\begin{array}{l}\text { Do not use when } \\
\text { answering a different } \\
\text { question }\end{array}$ & $\begin{array}{l}\text { "For me the strength was } \\
\text { that you came into the } \\
\text { project understanding } \\
\text { that if you really are } \\
\text { going to accompany the } \\
\text { community and really } \\
\text { hear from them, as we } \\
\text { did with our focus } \\
\text { group, it was about the } \\
\text { questions you came with } \\
\text { but then we started to } \\
\text { talk about things outside } \\
\text { that as well as how and } \\
\text { which they intersect" }\end{array}$ \\
\hline $\begin{array}{l}\text { Needs: Stigma/ } \\
\text { Normalize }\end{array}$ & $\begin{array}{l}\text { When participants talk } \\
\text { about the fact that the } \\
\text { project removed stigma }\end{array}$ & $\begin{array}{l}\text { Keywords: Stigma, } \\
\text { normalize }\end{array}$ & $\begin{array}{l}\text { Do not use when } \\
\text { answering a different }\end{array}$ & $\begin{array}{l}\text { "You really helped } \\
\text { remove the stigma } \\
\text { around it...I think what }\end{array}$ \\
\hline
\end{tabular}




\begin{tabular}{|c|c|c|c|c|}
\hline & $\begin{array}{l}\text { and normalized mental } \\
\text { health for people }\end{array}$ & & question & $\begin{array}{l}\text { the project did was } \\
\text { normalize once again, } \\
\text { it's in everybody's } \\
\text { family. Was there } \\
\text { anyone there that didn't } \\
\text { own up to, including the } \\
\text { participants as the } \\
\text { professionals, if you } \\
\text { will, own up to having } \\
\text { had mental health issues. } \\
\text { Either had, or currently } \\
\text { living with" }\end{array}$ \\
\hline Needs: Resources & $\begin{array}{l}\text { When participants talk } \\
\text { about the project as } \\
\text { providing resources for } \\
\text { people in the community } \\
\text { who may need them } \\
\text { When participants talk } \\
\text { about how this project } \\
\text { will teach people in } \\
\text { other frontline areas } \\
\text { (e.g., Social Work) to } \\
\text { help them better address } \\
\text { mental health for } \\
\text { Aboriginal people }\end{array}$ & Keywords: Resources & $\begin{array}{l}\text { Do not use when } \\
\text { answering a different } \\
\text { question }\end{array}$ & $\begin{array}{l}\text { "You offered potential } \\
\text { places of support where } \\
\text { people could go for free } \\
\text { support around mental } \\
\text { health issues" } \\
\text { "...this could be a good } \\
\text { guide for social workers } \\
\text { and caregivers coming } \\
\text { up because they will } \\
\text { realize the massive } \\
\text { challenges out there but } \\
\text { they can still be } \\
\text { overcome. Even one } \\
\text { little issue, if talked } \\
\text { about a resolution can } \\
\text { come about. They know } \\
\text { this is the issue, here's } \\
\text { what I can do to take }\end{array}$ \\
\hline
\end{tabular}




\begin{tabular}{|c|c|c|c|c|}
\hline & & & & $\begin{array}{l}\text { care of it and they take } \\
\text { care of it and they go } \\
\text { on" }\end{array}$ \\
\hline $\begin{array}{l}\text { Needs: Unsure/Don’t } \\
\text { Know }\end{array}$ & $\begin{array}{l}\text { When participants } \\
\text { express that they don't } \\
\text { know or are unsure. }\end{array}$ & $\begin{array}{l}\text { Use this code if they } \\
\text { 'guess' how it may have } \\
\text { addressed community } \\
\text { needs } \\
\text { Include reasons that they } \\
\text { may provide if they are } \\
\text { unsure }\end{array}$ & $\begin{array}{l}\text { Do not use this code if } \\
\text { they answer with } \\
\text { certainty }\end{array}$ & $\begin{array}{l}\text { "I'm not going to be able } \\
\text { to answer this question. } \\
\text { Only because I always } \\
\text { want to answer you } \\
\text { honestly and I'm not } \\
\text { around right this second } \\
\text { to see" }\end{array}$ \\
\hline \multicolumn{5}{|l|}{ Other General Codes } \\
\hline KI Impacted & $\begin{array}{l}\text { When participants talk } \\
\text { about how they were } \\
\text { impacted by the project } \\
\text { in some way }\end{array}$ & $\begin{array}{l}\text { Use this code if someone } \\
\text { talks about how this } \\
\text { project affected them by } \\
\text { either changing the way } \\
\text { they thought about } \\
\text { things or motivating } \\
\text { them in some way }\end{array}$ & $\begin{array}{l}\text { Do not use if they are } \\
\text { not talking about how } \\
\text { the overall project } \\
\text { impacted them in any } \\
\text { way }\end{array}$ & $\begin{array}{l}\text { "...it's actually taught } \\
\text { me. I'll tell you the truth. } \\
\text { Your dedication to your } \\
\text { studies has taught me } \\
\text { that in some ways I need } \\
\text { to do a PhD as well" }\end{array}$ \\
\hline Miscellaneous & & $\begin{array}{l}\text { Anything that doesn't go } \\
\text { under the other codes }\end{array}$ & & \\
\hline
\end{tabular}

Guidelines to keep in mind when coding:

(1) You don't have to code everything

(2) Be sure to include the Is, P1s, P2s, etc when you code

(3) Keep track of questions or uncertainties when you code so that we can clarify them when we chat next

(4) Try not to code identifying information 


\section{Appendix $U$ \\ Wellbeing Activity Booklet Handout \\ Wellbeing and Being Well: \\ Information and Coping Strategies to Promote Wellbeing \\ Date: February $1^{\text {st }}, 2014$ (Oakham Lounge) \\ 10:00am to $4: 30 \mathrm{pm}$ \\ Agenda}

10:00am to $10: 30 \mathrm{am}$

10:30am to $11: 00 \mathrm{am}$

11:00am to 11:30am

11:30am to $12: 30 \mathrm{pm}$

$12: 30 p m$ to $1: 15 p m$

$1: 15 \mathrm{pm}$ to $3: 00 \mathrm{pm}$

3:00pm to $3: 15 p m$

$3: 15 \mathrm{pm}$ to $3: 45 \mathrm{pm}$

$3: 45$ to $4: 15 \mathrm{pm}$

4:15 to $4: 30 \mathrm{pm}$
-Opening \& welcome -Introductions

-Four Questions

-Jeff D'Hondt Presentation (Native Child and Family Services)

-Wellbeing discussion: How do we view wellbeing and what impacts wellbeing)?

-Information sharing about wellbeing issues (e.g., anxiety and depression) \& mental health terminology

\section{Catered lunch (Indian Tacos)}

-Traditional teaching from Joanne Dallaire

-Discussion: Coping Strategies \& How to promoting others' wellbeing

Break

-Nicole Penak Presentation (Anishnawbe Health)

-Treatment options

-Navigating the mental health system

-Final thoughts \& Closing

-Post-measure \& Feedback form 


\section{Justice Sinclair's Four Questions}

Ask yourself.....

\section{Who am I?}

\section{Where am I from?}

Where am I going?

What am I here to do? 


\section{QUESTIONS YOU MAY ASK}

1. What is this medication typically prescribed for?

2. Can you go over the possible side effects with me?

*Note: you will receive a written list of side effects, but it is often helpful for your doctor or pharmacist to go over them with you (especially the common ones)

3. Are there any medications that I should not be taking in combination with this medication?

4. Are there any websites you would recommend to me where I can gather more information on this medication?

*Note: if you type the name of your medication into Google, you will be able to find different sources to gather information on your medications (it is recommended that you look on at least three websites, so that you can increase the likelihood that you are receiving correct and the most up-to-date information)

5. Are there any treatment options for me other than medication?

*Note: this question is not intended to discount the help that medications can provide you with, it is just for you to have all of the information

6. Where should I go or what should I do if I experience a serious side effect or a reaction of some sort?

7. Are there any special instructions for this medication (e.g., do I need to take this with food or water)?

Other questions that I could ask: 


\section{Coping Ideas: From your Peers}

- Go to the gym or exercise on your own: walk, run, bike, etc.

- Talk to family and friends for support

- Read about whatever you may be going through (e.g., look up concerns online)

- Watch TV

- Sleep

- Write in a journal

- Pray

- Meditate

- Have a hot bath, shower, or sauna

- Read a book, newspaper, or magazine

- Cry

- Eat your favorite food

- Get or give a massage

- Socialize with others

- Laugh or make others laugh

- Play a board game

- Listen to music

- Make music

- Garden

- Play with your pet

- Splash cold water on your face

- Talk to an Elder,

- Go on a nature hike

- Go to a sweat lodge ceremony

- Turn to traditional medicines

- Pray/give thanks to the Creator

- Make a drum

- Drum and/or sing traditional songs

- Bead

- Deal with mental health issues within the family

- Smudge

- Connect to the land (e.g., by going to a park or going back to your Aboriginal community)

- Turning to your ceremonies.

- Elders and healers can work with those who have more severe mental health issues

- Bring different caregivers together to address all dimensions of the self

- **Go to the Emergency Room or check yourself into an inpatient unit if there is concern that you may harm yourself***

Other ideas for yourself 


\section{Community Resource List - TORONTO AREA}

\section{REFERAL SERVICES}

\section{Canadian Mental Health Association - Metro Branch}

416-535-8501

www.ontario.cmha.ca

Information about individual psychiatrists, listed by speciality area (affective disorders, schizophrenia), location and languages spoken.

\section{Church Street Community Centre}

416-392-6874

www.the519.org

Counseling referral service for lesbians and gays. Does provide free in house counselling (up to six sessions). Some services offered through this organization require a fee, but all are sliding scale.

\section{Guide to Social Services}

211

www.211 toronto.ca

\section{National Eating Disorders Information Centre (NEDIC)}

416-340-4156

416-408-4357 (distress centre)

1-866-NEDIC-20

www.nedic.ca

Information and referrals to therapists and services.

\section{Ontario Problem Gambling Hotline}

1-888-230-3505

Toll free, confidential, anonymous service helplines, by accessing website you can be connected with an Information and Referral Specialist for a list of available treatment centres in your area, 24 hours, 7 days per week.

\section{SERVICES TARGETING ABORIGINAL PEOPLES}

\footnotetext{
Aboriginal Services Program: Centre for Addiction and Mental Health

60 White Squirrel Way, 2nd Floor

416-535-8501 ext 33987 (information)

www.camh.net

Provides individual and group counselling to Aboriginal people experiencing homelessness, substance abuse and mental health issues. Group programs include a 21-day treatment cycle for men, a 21-day treatment cycle for women, a 6-week outpatient cycle for both genders. Other services include: telephone Elder visits, case consultation and education. No referral required and no fee required.
} 
Anishnawbe Health Toronto

Gerrard: 416-920-2605

Queen: 416-360-0486 (Medical Unit)

Vaughn: 416-657-0379 (Mental Health and Addiction Unit)

Free services (need OHIP to access medical services)

www.aht.ca

Offers support services and counselling and aims to improve the health and wellbeing of

Aboriginal people in spirit, mind, emotion, and body by providing traditional healing within a multidisciplinary healthcare model. Free service, slef-referral, and require OHIP for medical

services only.

\section{2-Spirited People of the $1^{\text {st }}$ Nation}

145 Front Street East

416-944-9300 ext 222

www.2spirits.com

A non-profit social services organization whose membership consists of Aboriginal gay and lesbian, bisexual and transgendered people in Toronto and services include HIV/AIDS education, outreach, prevention, support, and counselling

\section{Council Fire Native Cultural Centre}

439 Dundas Street East

416-360-4350

http://www.councilfire.ca/

Provides counselling, material assistance and other direct social services to First Nations people and to encourage and enhance spiritual and personal growth.

\section{Dodem Kanonhsa'}

55 St. Clair Avenue East, 6th Floor

416-952-9272

http://www.dodemkanonhsa.ca/index.htm

Promote sharing and understanding of Aboriginal culture and its philosophies. Elders and Teachers conduct traditional ceremonies and provide teachings and cultural counselling, called ONE-to-ONE sessions.

\section{Indian Residential Schools Resolution Health Support Program}

1-888-301-6426 (call for information and referral)

1-866-925-4419 (24-Hour National Crisis Line)

Provides one-on-one counselling for residential school survivors and their families. Contact the information and referral line to provide name, birth date as well as residential school attended and years attended (if aware of this). Client will be provided with a list of counsellors. Free service and will be reimbursed for travel expenses.

\section{Native Canadian Centre of Toronto}

16 Spadina $\mathrm{Rd}$

416-964-9087 
http://ncct.on.ca

Offers Aboriginal Education Outreach Program, Communications and Referrals, and Cultural Programs.

\section{Native Child and Family Services of Toronto}

30 College Street

416-969-8510

www.nativechild.org

Offers support services that deal with families in a holistic and culturally sensitive way and have many regular drop in programs daily.

\section{Native Men's Residence (NA-ME-RES)}

14 Vaughan $\mathrm{Rd}$

416-652-0334

www.nameres.org

Provides services to homeless men 16 years and older. multi-disciplinary care team including traditional elders and teachers, psychiatrist, nurse. Provides case management, counselling, stress management, legal information, advocacy, housing assistance. Referral from other Aboriginal or social service agency and no fee required.

\section{Native Women's Resource Centre}

191 Gerrard St East

416-963-9963

http://www.nativewomenscentre.org/welcome.html

Offers traditional programming and Counseling Services by appointment through CAMH and Aboriginal Care Team. Free services and no referral required. Call to make an appointment.

\section{Ontario Aboriginal HIV/Aids Strategy}

7 Hayden St. Ste 201

416-944-9481

www.oahas.org

The Aboriginal HIV/AIDS strategy provides culturally sensitive and respectful programs to respond to the growing number of Aboriginal people with HIV/AIDS, including counselling and long-term care-support groups. Services are free and no referral is required.

\section{CRISIS SERVICES}

\section{Assaulted Women's Helpline}

416-863-0511 (crisis line) 1-866-863-0511 (Crisis, outside of GTA), 416-364-4144 (Admin line) www.awhl.org

Confidential telephone service providing crisis counselling, emotional support, safety planning, and information and referrals for emergency shelters, counselling legal services and other community services. Services is free.

\section{Distress Centres of Toronto}


416-408-4357

www.torontodistresscentre.com

Immediate emotional support, crisis intervention and suicide prevention over the phone. Open 24 hours, 7 days.

\section{Gerstein Centre Crisis Line}

416-929-5200

www.gersteincentre.org

Crisis intervention including phone line, mobile unit, ten-bed stay crisis house, and hospital referral. Staff provides supportive counselling for immediate crisis issues, and referrals to other services for ongoing, non-crisis issues. Serves people of Toronto.

\section{Lesbian, Gay, Bi, Trans Youth Line}

1-800-268-9688

www.youthline.ca

Toll-free service provided by youth for youth. Offers support, information and referrals specific to your concerns.

\section{St. Michael's Hospital Psychiatric Emergency Services}

416-808-2222

http://www.stmichaelshospital.com/programs/mentalhealth/emergency.php

Mobile crisis intervention team responds to 911 emergency and police dispatch calls involving emotionally disturbed individuals, from $1 \mathrm{pm}$ to $11 \mathrm{pm}$, seven days a week. Call 911 to request the crisis team’s services. Tel: 911 (Emergencies), 416-808-2222 (Non-emergencies)

\section{The Integrated Community Mental Health Crisis Response Program}

\section{6-498-0043}

http://www.saintelizabeth.com/Services-and-Programs/Services-for-Communities/Crisis-

Intervention.aspx

Serving residents of Etobicoke and North York, the Community Crisis Program, Metro Site, provides support to adults 16 years and older who are experiencing personal mental health challenges, concerns about family and friends, life adjustment and coping issues, issues of selfcare, seniors in crisis, and suicide risk. Service is free.

\section{Toronto Rape Crisis Centre/Multicultural Women Against Rape}

416-597-8808 (crisis) 416-597-1171 (admin line)

http://www.trccmwar.ca/

Crisis intervention, counselling and referral for survivors of rape/sexual assault. All services are free.

\section{Victims Services Program of Toronto}

416-808-7066 (crisis line)

www.victimservicestoronto.com

Provides immediate on-site crisis and trauma services for victims of crime or sudden tragic events, their families and witnesses. Self-referrals are accepted, must apply within 14 business days after crime is reported to police and there is no fee required. 


\section{COUNSELLING SERVICES}

\section{Alternatives: East York Mental Health Counselling Services Agency \\ 1245 Danforth Avenue \\ 416-285-7996 \\ Persons 16 years and older with serious, longstanding mental health problems. Self-referral encouraged and no fee.}

\section{Barbara Schlifer Clinic}

489 College Street

416-323-9149

www.schliferclinic.com

Individual and group counselling for women who have experienced adult sexual assault, intimate partner abuse, incest, childhood sexual abuse. Free legal advice and consultation. Referrals to lawyers, help with the justice system. Counselling available in English, Albanian, Italian, Spanish, Hindi, Punjabi and Urdu.

\section{Catholic Family Services Toronto}

1155 Yonge Street, Suite 200

416-921-1163

www.cfsofto.org

Offers Individual, Couple, Family and Group counselling. Fees based on income level. Waiting list varies.

\section{Central Toronto Community Health Centre}

168 Bathurst Street

416-703-8480 ext. 400

http://www.ctchc.com/site qw/programs.html

We offer individual and group counseling to clients living in the Queen West catchment area who are dealing with personal, emotional or mental health and addictions concerns. We also work with parents of young children age 0 to 6 . There may be a waiting list. No fee for counselling and no referral required.

Counselling and Psychoeducational clinic at the Ontario Institute for Studies in Education of the University of Toronto

252 Bloor Street West

416-978-0620

http://clinic.oise.utoronto.ca/

Provides psychological assessment, counseling, and psychotherapy services to adults and older adolescents for personal, social, emotional, and academic concerns. Available services vary depending on the OISE/UT academic schedule; therefore, not all services are available at all times throughout the year. Flexible payment plans and sliding fee scale are offered for services not covered by extended health plan.

\section{East Toronto Health Centre}


1496 Danforth Ave

416-778-1496

No website

A private community mental health setting. Psychiatrists and GP psychotherapists provide consultation, assessment and individual and family psychotherapy. Requires a referral from your family physician and is OHIP covered.

Family Service Association of Toronto

355 Church Street

416-595-9618/416-595-9230

www.fsatoronto.com

Provides professional counselling for individuals and families concerned about family relationships and conflict, coping/managing feelings, problem solving, relationship building, the impact on life changes and childhood trauma. Also provides community connections and support. Services available in English, Spanish and Farsi. Some services are free and fees are based on family size and income and begin at $\$ 10$ per hour.

Progress Place

576 Church St

416-323-0223

www.progressplace.org

Adults 18-65 years with significant mental health problems, Community-based psychosocial rehabilitation centre, based on the clubhouse approach. Professional or self-referral and no fee required.

\section{Toronto Institute for Relational Psychotherapy}

1046 - 7 B Pleasant Boulevard

416-465-2392

www.tirp.ca

Low cost psychotherapy to the general public by advanced students in training. Relational therapy with recent grads of the Institute is also available at $\$ 25 /$ session for up to 20 sessions. No referral required.

\section{SPECIALIZED CLINICS}

\section{Bellwood Health Services}

$1020 \mathrm{McNicoll}$ Ave

416-495-0926 (office), 416-495-0926 (crisis)

www.bellwood.ca

Residential and outpatient addiction treatment, including individual, family and couples counselling. Contact intake worker for an assessment. Fee for service for some programs and others require OHIP.

\section{Centre for Addiction and Mental Health}

Location varies by service 416-535-8501, extensions vary by service 
www.camh.net

Canada's largest mental health and addiction hospital. CAMH combines clinical care, research, education, policy development and health promotion to help transform the lives of people affected by mental health and addiction issues. Services include, Mood and Anxiety program, Addiction Services, Schizophrenia Services, Forensic Services, Women's Services. Some services require a referral from a GP or psychiatrist and all services are covered by OHIP.

Jean Tweed Centre

215 Evans Ave

416-255-7359

www.jeantweed.com

Women 16 years and older who have problems with alcohol, other drugs or problem gambling all services available to women on methadone. Professional or self-referral required and services are free.

\section{Women's College Hospital-Sexual Assault and Domestic Violence Care Centre} 76 Grenville Street

416-323-6040

http://www.womenscollegehospital.ca/programs-and-services/sexual-assault-domestic-violencecare-centre/

Provides medical treatment and psychological support to victims of domestic violence or sexual assault. There is no fee for the service. Only see patients who were seen by women's college hospital after a sexual assault.

Medical Clinic for Person-Centered Psychotherapy

265 Yorkland Blvd., North York

416-229-2399 or 1-888-229-8088

www.medicalpsychclinic.org

Provides psychotherapy and counselling services for people dealing with issues such as depression, anxiety, grief, trauma, etc. Individual psychotherapy is available as well as couple counselling and group therapy. Ages 18+. Requires a referral from GP or psychiatrist.

\section{Sheena's Place}

87 Spadina Road

416-927-8900

www.sheenasplace.org

Support programs and groups services for those with eating disorders and related issues, their families and caregivers. No referral needed. No fee required.

\section{Distress Centre Toronto - Survivor Support Programme}

Address is not advertise

416-595-1716

https://www.torontodistresscentre.com/survivor-support

Provides individualized grief support for survivors (bereaved family and friends) of suicide and homicide loss in safe, one on one formats, provides follow-up series of group support sessions. No fee required and self-referrals are accepted. 


\title{
Women Recovering from Abuse Program (WRAP)
}

\section{Grenville Street}

416-323-6400 x4863

http://www.womenscollegehospital.ca/programs/program126.html

Offers group therapy and individual counselling to women who have experienced abuse, who suffer from mental health problems, and who have sought traditional forms of psychiatric treatment in the past. Accepts self-referrals, as well as referrals from health care professionals. Covered by OHIP.

\section{Bay Centre for Birth Control 790 Bay Street \\ 416-351-3700 \\ www.womenscollegehospital.ca/programs/program63.html}

Provides contraceptive health care, reproductive health care, abortion and sexual health care, as well as services related to Hepatitis B and HIV (i.e., testing, counselling). No referral required and covered by OHIP.

\section{SUPPORT/SELF HELP GROUPS}

\section{Al-Anon and Alateen Family Groups \\ 416-410-3809 \\ al-anon.alateen.on.ca \\ Provides support group meetings for relatives and friends of alcoholics. Visit website for times and locations, as they will vary across the city.}

\author{
Alcoholics Anonymous \\ Office: 416-487-5591 or 1-877-404-5591 (toll free in Ontario) \\ Crisis: 416-487-5591 or 1-877-404-5591 (toll free in Ontario) \\ www.aatoronto.org \\ Support group for recovering alcoholics. Call to find group in your area.
}

\section{Emotions Anonymous}

Monday meeting 647-283-0258; Friday meeting 416-535-9147

www.emotionsanonymous.org

A 12 step self-help group for anyone with emotional problems such as fear, anxiety, depression, anger, and grief.

\section{Mood disorders Association of Ontario}

416-486-8046 (support line), 1-888-486-8236 (toll-free)

www.mooddisorders.on.ca

Telephone information support and self-help peer support groups, which share experiences and coping techniques. Support groups held in various locations. No fee required. Fact sheets about mood disorders are available on the website.

\section{Obsessive-Compulsive Disorder Network}


416-410-4722

www.ocdontario.org

All volunteer, non-profit charity, focused on providing help and information to children and adults with OCD and their families. Website also provides a directory of services.

Victim Support Line (VSL)

416-314-2447/1-888-579-2888

Offers information and referral services in your community, as well as information about the criminal justice system and access to information about provincially sentenced offenders.

Services offered in 13 different languages.

\section{WRITTEN RESOURCES}

Abramowitz, J. S. (2009). Getting over OCD: A 10-step workbook for taking back your life. New York, NY: The Guilford Press.

Antony, M. M. \& Swinson, R. P. The shyness \& social anxiety workbook. Oakland, CA: New Harbinger Publications Inc.

Bourne, E. J. (2010). The anxiety \& phobia workbook ( $5^{\text {th }}$ ed). Oakland, CA: New Harbinger Publications Inc.

Carney, C. E. \& Manber, R. (2009). Quiet your mind \& get to sleep. Oakland CA: New Harbinger Publications Inc.

Davis, M, Eshelman, E. R., \& McKay, M. (2008). The relaxation and stress reduction workbook $\left(6^{\text {th }}\right.$ ed.). Oakland, CA: New Harbinger Publications Inc.

Greenberger, D. \& Padesky, C. A. (1995) Mind over mood: Change how you feel by changing the way you think. New York, NY: The Guilford Press.

\section{WEBSITES}

www.supportgroups.com

Www.sixbillionsecrets.com

www.dailystrengths.org

http://www.anxietybc.com/resources/selfhelp_home toolkit.php

http://www.anxieties.com/

http://www.helpguide.org/mental/depression_tips.htm

*It is recommended that you look at three different website when gathering self help information to increase the likelihood that you are collecting accurate and the most up-todate information 


\section{Appendix $V$ \\ Facilitator's Version of Wellbeing Activity Agenda \\ Wellbeing and Being Well: \\ Information and Coping Strategies to Promote Wellbeing \\ Sharing Circle \\ Date: February $1^{\text {st }}, 2014$ (Oakham House - 63 Gould Street) \\ 10:00am to $4: 30 \mathrm{pm}$}

\section{Agenda (Facilitator's Version)}

10:00am to $10: 30 \mathrm{am}$

$10: 30$ to $11: 30$

(Caitlin to begin Discussions)

$11: 30$ to $12: 30 \mathrm{pm}$

(Caitlin to begin Discussion)

$12: 30$ to $1: 15 \mathrm{pm}$

1:15 to $3: 00 \mathrm{pm}$

(Joanne to begin Discussion)

3:00pm to $3: 10 \mathrm{pm}$
Opening (Joanne) \& welcome

Go through Agenda (Caitlin)

Consent \& premeasured (Caitlin)

Introductions (Caitlin \& Joanne begin)

Normalizing mental health (Joanne)

Ask yourself: Who am I? Where am I from? Where

am I going? What am I here to do? (Handout) (Caitlin)

How do you think cultural connection plays a role in wellbeing? (Some info from D'Hondt on this topic and Native Child)

How do people view wellbeing?

How do you think colonization impacts wellbeing?

How does identity impact wellbeing?

What does mental health stigma mean to you?

What does depression mean to you?

What does anxiety mean to you?

What does addiction mean to you?

What does suicide mean to you?

What does it mean to have an eating disorder?

What does it mean to have schizophrenia?

Mental health terminology: How you can speak about mental health and what terminology mental health professionals use?

Handout: Where to get more information

Catered lunch (Indian Tacos)

Traditional teaching from Joanne Dallaire

Overview of helpful and unhelpful coping strategies

Discussion: How to promoting others' wellbeing

Handout: Coping Strategies

Break 
$3: 15$ to $4: 15 \mathrm{pm}$

$4: 15$ to $4: 30 \mathrm{pm}$
Navigating the mental health system and info about Anishnawbe Health (Panek to present)

Treatment options

Questions to ask when accessing treatment Things to keep in mind about the system

Handout: Resource List and Medication Info Handout

Closing (Joanne)

Post-measure \& Feedback form 


\section{References}

Aboriginal Affairs and Northern Development Canada. (2006). Fact sheet - Urban Aboriginal population in Canada. Ottawa, ON: Aboriginal Affairs and Northern Development Canada.

Aboriginal Healing Foundation. (2003a). Aboriginal domestic violence in Canada. Ottawa, ON: Aboriginal Healing Foundation.

Aboriginal Healing Foundation. (2003b). Aboriginal people, resilience and the residential school legacy. Ottawa, ON: Aboriginal Healing Foundation.

Aboriginal Healing Foundation. (2005). Reclaiming connections: Understanding residential school trauma among Aboriginal People—A resource manual. Ottawa, ON: Aboriginal Healing Foundation.

Aboriginal Healing Foundation. (2007). Addictive behaviours among Aboriginal people in Canada. Ottawa, ON: Aboriginal Healing Foundation.

Aboriginal Healing Foundation. (2008). Aboriginal healing in Canada: Studies in therapeutic meaning and practice. Ottawa, ON: Aboriginal Healing Foundation.

Aboriginal Services. (2014). Aboriginal services. Retrieved from http://www.ryerson.ca/aboriginal/

Alberta Education. (2002). First Nations, Métis and Inuit (FNMI) Education Policy Framework. Edmonton, AB: Alberta Education.

Alberta Mental Health Board. (2006). Aboriginal mental health: A framework for Alberta. Alberta: Alberta Health Services.

Alberta Teacher's Association. (2006). Education is our buffalo. Edmonton, AB: Alberta Teachers' Association. 
American Psychiatric Association. (2000). Diagnostic and statistical manual of mental

disorders, fourth edition, text revision. Washington, DC: American Psychiatric Association.

American Psychological Association. (1992). Ethical principles of psychologists and code of conduct. American Psychologist, 47, 1597-1611.

Anishnawbe Health Toronto (2000a). Sacred items and bundles. Toronto, ON: Anishnawbe Health Toronto.

Anishnawbe Health Toronto (2000b). Sweat lodge. Toronto, ON: Anishnawbe Health Toronto.

Anishnawbe Health Toronto (2011). Anishnawbe Health Toronto. Retrieved from http://www.aht.ca/.

Archer, M., Bhasker, R., Collier, A., Lawson, T., \& Norrie, A. (1998). Critical realism: Essential readings. New York, NY: Routledge.

Asch, M. (2002). Self-government in the new millennium. In J. Bird, L. Land, \& M. MacAdam (Eds.), Nation to nation: Aboriginal sovereignty and the future of Canada. Scarborough, ON: Irwin Publishing Inc.

Assembly of First Nations. (2007). Sustaining the caregiving circle: First Nations people and aging. Ottawa, ON: Assembly of First Nations.

Barwick, M., Butterill, D., Lockett, D. M., Buckley, L., \& Goering, P. (2005). Scientist knowledge translation training manual (SKTT). Toronto, ON: The Hospital for Sick Children \& Centre for Addiction and Mental Health.

Baskin, C. (2005). Circles of inclusion: Aboriginal world views in social work education. (Unpublished doctoral dissertation). University of Toronto, Canada. 
Baskin, C. (2011). Strong helpers' teachings: The value of Indigenous knowledges in the helping professions. Toronto, ON: Canadian Scholars Press.

Battiste, M., \& Henderson, J. Y. (2000). Protecting Indigenous knowledge and heritage: A global challenge. Saskatoon, SK: Purich Publishing Ltd.

Battiste, M. (2000). Maintaining Aboriginal identity, language, and culture in modern society. In M. Battiste (Ed.), Reclaiming Indigenous voice and vision. (pp. 192-208). Vancouver, BC: University of British Columbia Press.

Baum, F., MacDougall, C., \& Smith, D. (2006). Participatory action research. Journal of Epidemiology and Community Health, 60, 854-857. doi:10.1136/jech.2004.028662

Bell Canada. (2014). Bell let's talk. Retrieved from http://letstalk.bell.ca/en/.

Benoit, C., Carroll, D., \& Chaudhry, M. (2003). In search of a healing place: Aboriginal women in Vancouver's downtown eastside. Social Science \& Medicine, 56, 821-833.

Bigfoot, D. S. \& Schmidt, S. R. (2010). Honoring children, mending the circle: Cultural adaptation of trauma focused cognitive-behavioral therapy for American Indian and Alaskan Native children. Journal of Clinical Psychology, 66, 847-856. doi:

$10.1002 /$ jclp. 20707

Blackstock, C. (2008). Rooting mental health in an Aboriginal worldview. The Provincial Centre of Excellence for Child and Youth Mental Health at CHEO.

Bougie, E., Wright S. C., \& Taylor, D. M. (2003). Early heritage-language education and the abrupt shift to a dominant-language classroom: Impact on the personal and collective esteem of Inuit children in Arctic Quebec. International Journal of Bilingual Education and Bilingualism, 6, 349-373. doi: 1367-0050/03/05 0349-24

Braun, V., \& Clarke, V. (2006). Using thematic analysis in psychology. Qualitative Research in 
Psychology, 3, 77-101.

Brave Heart, M. Y. H. (2003). The historical trauma response among natives and its relationship with substance abuse: A Lakota illustration. Journal of Psychoactive Drugs, 35(1), 7-13.

Brunnen, B. (2004). Working towards parity: Recommendations of the Aboriginal Human Capital Strategies Initiatives. Building the New West Report \#24. Calgary: Canada West Foundation.

Burr, V. (2003). Social constructionism. New York: Routledge Taylor \& Francis Group.

Cain, R., Jackson, R., Prentice, T., Mill, J., Collins, \& Barlow, K. (2011). Depression among Aboriginal people living with HIV in Canada. Canadian Journal of Community Mental Health, 30 (1), 105-120.

Canadian Institutes of Health Research. (2009). Aboriginal knowledge translation:

Understanding the distinct needs of Aboriginal communities in research. Ottawa, ON: Canadian Institutes of Health Research.

Canadian Institutes for Health Research. (2011). CIHR guidelines for health research involving Aboriginal people. Ottawa, ON: Canadian Institutes for Health Research.

Canadian Population Health Initiative (2009). Mentally healthy communities: Aboriginal perspectives. Ottawa, ON: Canadian Institutes for Health Information.

Caplan, N. (1979). Two communities theory and knowledge utilization. American Behavioural Scientist, 22, 459-470.

Castellano, M. B. (2004). Ethics of Aboriginal research. Journal of Aboriginal Health.

Chandler, M. J., \& Lalonde, C. E. (1998). Cultural continuity as a hedge against suicide in Canada's First Nations. Transcultural Psychiatry, 35, 191-219.

Chandler, M. J., \& Lalonde, C. E. (2008). Cultural continuity as a protective factor against 
suicide in First Nations youth. Horizons - A Special Issue on Aboriginal Youth, Hope or Heartbreak: Aboriginal Youth and Canada's Future. 10(1), 68-72.

Chouinard, J. A. \& Cousins, J. B. (2007). Culturally competent evaluation for Aboriginal communities: A review of the empirical literature. Journal of Multidisciplinary Evaluation, 4, 40-57.

Christie, L., \& Halpern, J. M. (1990). Temporal constructs and Inuit mental health. Social Science and Medicine, 30, 739-749.

Clarke, V. (2005). 'We're all very liberal in our views': Students' talk about lesbian and gay parenting. Lesbian and Gay Psychology Review, 6, 2-15.

Collier, A. W. (1994). Critical realism: An introduction to Roy Bhaskar's Philosophy. New York, NY: Verso.

Constitution Act. (1982). Section 35 (2).

Connors, E. A., (2007). Intergenerational trauma and healing. Presented at the Association of the Canadian Court Administrators Conference.

Cornwall, A., \& Jewkes, R. (1995). What is participatory research? Social Science \& Medicine, 41, 794. doi:10.1016/0277-9536(95)00127-S

Crabtree B. F., \& Miller, W. L. (1999). Doing qualitative research. Thousand Oaks, CA: Sage Publications.

Danermark, B., Ekstrom, M., Jakobson, L., Karlsson, J. C. (2002). Explaining society: Critical realism in the social sciences. London, UK: Routledge.

Dell, C. Hopkins, C. \& Dell, D. (2005). Resiliency and holistic inhalant abuse treatment. Journal of Aboriginal Health, 2 (1), 4-12.

Dell, C. A., Seguin, M., Hopkins, C., Tempier, R., Mehl-Madrona, L., Dell, D. et al. (2011). 
From benzos to berries: Treatment offered at an Aboriginal youth solvent abuse treatment centre relays the importance of culture. Canadian Journal of Psychiatry, 56, 75-83.

Department of Finance. (2012). Bill C-45 - “Jobs and Growth Act, 2012” - Part 4. Ottawa, ON: Government of Canada.

Denzin, N. K., \& Lincoln, Y. S. (2005). The sage handbook of qualitative research. Thousand Oaks, CA: Sage Publications Inc.

Duffy, R. Q. (1988) The Road to Nunavut. Montreal: McGill-Queen's University Press.

Dumont-Smith, C. (2002). Aboriginal Elders Abuse in Canada. [No publisher listed].

Edge, L. \& McCallum, T. (n.d.). Métis identity: Sharing traditional knowledge and healing practices at Métis Elders' gatherings. Pimatisiwin, 83-115.

Enright, M., Maitland, A. (Interviewers), \& Fontaine, P. (Interviewee). (1990, November 5). Phil Fontaine opens up about abuse [Audio interview]. Retrieved from http://www.cbc.ca/archives/categories/politics/parties-leaders/phil-fontaine-nativediplomat-and-dealmaker/abused-to-abuser.html

Epstein, R. S. (1994). Keeping boundaries: Maintaining safety and integrity in the psychotherapeutic process. Washington, DC: American Psychiatric Press.

Erikson, E. (1970). The making of modern science: Biographical studies. Daedalus, 99, 730-759.

Evans, H. (2014). Aboriginal communities oppose land grab. Retrieved from: http://search.informit.com.au/documentSummary; $\mathrm{dn}=722931835015848 ;$ res=IELHSS.

Evans, M., Hole, R., Berg, L. D., Hutchinson, P., Sookraj, D. (2009). Toward a fusion of indigenous methodologies participatory action research and white studies in an urban Aboriginal research agenda. Qualitative Inquiry, 15, 893-910.

Finlay, J., Hardy, M., Morrs, D., \& Nagy, A. (2010). Mamow ki-ken-da-ma-win: A partnership 
approach to child, youth, family and community wellbeing. The International Journal of Mental Health and Addiction, 8, 245-257. doi: 10.1007/s11469-009-9263-8

Fisher, J. K. (2006). Adult daughters of incest survivors: Exploring the transgenerational legacy of trauma (Doctoral dissertation). Retrieved from http://search.proquest.com.ezproxy.lib.ryerson.ca/psycinfo/docview/304948439/previewP DF/3D4607BD44DF4DE3PQ/14?accountid=13631

Fletcher, C. (2003). Community-based participatory research relationships with Aboriginal communities in Canada: An overview of context and process. Pimatziwin: A Journal of Aboriginal and Indigenous Community Health 1(1), 27-62.

Flicker, S., Savan, B., Kolenda, B., \& Mildenberger, M. (2008). A snapshot of community-based research in Canada: Who? What? Why? How? Health Education Research, 23 (1), 106114.

Fox, D., Prilleltensky, \& Austin, S. (2011). Critical psychology: An introduction. London, UK: SAGE Publications Ltd.

Freemantle, N., Harvey, E. L., Wolf, F., Grimshaw, J. M., Grilli, R., Bero, L. A. (1995). Printed educational materials to improve the behaviour of health care professionals and patient outcomes (Cochrane Review). In: The Cochrane Library, issue 3. Oxford: Update Software, 1998. British Medical Journal, 311, 848-852.

Frideres, J. S. (1988) Native Peoples in Canada: Contemporary Conflicts. Englewood Cliffs, NJ: Prentice Hall.

Gergen, K. J. (1985). The social constructionist movement in modern psychology. American Psychologist, 40, 266-275.

Gergen, K. J. (2009). An invitation to social construction. London, UK: SAGE Publications Ltd. 
Goldstein, E. G. (1994). Self-disclosure in treatment: What therapists do and don't talk about. Clinical Social Work Journal, 22, 417-433.

Government of Canada (2012). Research involving First Nations, Inuit and Métis Peoples of Canada (Chapter 9). In Tricouncil Policy Statement: Ethical Conduct for Research Involving Humans. Ottawa, ON: Government of Canada.

Graham, I. D., Logan, J., Harrison, M. B., Straus, S. E., Tetroe, J., Caswell, W. et al. (2006). Lost in knowledge translation: Time for a map? Journal of Continuing Education in the Health Profession, 25 (1), 13-24. doi: 10.1002/chp.47

Grilli, R., Freemantle, N., Minozzi, S., Domenighetti, G., \& Finer, D. (2000). Mass media interventions: Effects on health services utilisation. Cochrane Database Systematic Review, 2.

Grimshaw, J., Eccles, M., Walker, A., \& Thomas, R. (2002). Changing physician's behaviour: What works and thoughts on getting more things to work. Journal of Continuing Education in the Health Professions, 22, 237-43.

Grol, R. \& Grimshaw, J. (2003). From best evidence to best practice: Effective implementation of change in patients' care. Lancet, 362, 1170.

Gone, J. P. (2006). Mental health, wellness, and the quest for an authentic American Indian identity. In T. Witko (Ed.), Mental health care for urban Indians: Clinical insights from Native practitioners. (pp. 55-80). Washington, DC: American Psychological Association.

Gone, J. P. (2007). “We never was happy living like a whiteman”: Mental health disparities and the postcolonial predicament in American Indian communities. American Journal of Community Psychology, 40, 290-300. doi: 10.1007/s10464-007-9136-x

Gone, J. P. (2009). A community-based treatment for Native American historical trauma: 
Prospects for evidence-based practice. Journal of Consulting and Clinical Psychology, 77, 751-762. doi: 10.1037/a0015390

Gone, J. P. (2011). The red road to wellness: Cultural reclamation in a Native First Nations community treatment center. American Journal of Community Psychology, 27, 187-202. doi: $10.1007 / \mathrm{s} 10464-010-9373-2$

Gray, D., Saggers, S., Sputor, B., \& Bourbon, D. (2000). What works? A review of evaluated alcohol misuse interventions among Aboriginal Australians. Addiction, 95(1), 11-22.

Hacking, I. (1999, May/June). Are you a social constructionist? Lingua Franca, pp. 65-72.

Hall, B. (1993). Introduction. In P. Park, M. Brydon-Miller, B. Hall, T. Jackson et al. (Eds). Voices of change: Participatory research in the United States and Canada. (pp. xiiixxii). Toronto, ON: OISE Press.

Hallett, D. (2005). Aboriginal identity development, language knowledge, and school attrition: an examination of cultural continuity (Unpublished doctoral dissertation). University of British Columbia, Vancouver, BC.

Hanson, P. G., \& Smylie, J. (2006). Knowledge translation for Indigenous communities: A policy making toolkit.

Harding, G.S. (2005). Surviving In the Hour of Darkness: The Health and Wellness of Women of Colour and Indigenous Women. Calgary, AB: University of Calgary Press.

Hare, J. \& Barman, J. (2000). Aboriginal education: Is there a way ahead? In Long. D \& Dickenson, O, (Eds.), Vision of the heart: Canadian Aboriginal issues. (pp. 331-359). Toronto: Harcourt Canada Ltd.

Hartmann, W. E., \& Gone, J. P. (2012). Incorporating traditional healing into an urban American Indian health organization: A case study of community member perspective. Journal of 
Counseling Psychology, 59, 542-554. doi: 10.1037/a0029067

Hart, M. (2007). Indigenous knowledge and research: The mikiwahp as a symbol for reclaiming our knowledge and ways of knowing. First Peoples Child and Family Review, 3(1), 8390.

Health Canada. (2003). Acting on what we know: Preventing youth suicide in First Nations. Ottawa, ON: Advisory Group on Suicide Prevention.

Health Canada. (2010). Working together to improve Aboriginal access to health services. Ottawa, ON: Health Canada.

Hecker, R. (1997). Participatory action research as a strategy for empowering Aboriginal health workers. Australian and New Zealand Journal of Public Health, 21, 784-788.

Hepburn, A., \& Jackson, C. (2009). Rethinking subjectivity: A discursive psychological approach to cognition and emotion. In D. Fox, I. Prilleltensky \& S. Austin (Eds.), Critical psychology: An introduction. (pp. 176-194). London, UK: SAGE Publications Ltd.

Heron, J., \& Reason, P. (1997). A participatory inquiry paradigm. Qualitative Inquiry, 3, 274294.

Hinshaw, S. P. (2007). The mark of shame: Stigma of mental illness and an agenda for change. New York, NY: Oxford University Press.

Holmes, D. (2006). Redressing the balance: Canadian University programs in support of Aboriginal students. Ottawa, ON: Association of Universities and Colleges Canada.

Horvath, A. O., \& Lester, L. (1993). The role of the therapeutic alliance in psychotherapy. Journal of Consulting and Clinical Psychology, 61, 561-573.

Humphrey, K. (2001). Dirty questions: Indigenous health and 'Western research". Australian and New Zealand Journal of Public Health, 25, 197-202. 
Idle No More. (no date). Idle no more. Retrieved from http://www.idlenomore.ca/.

Indian and Northern Affairs Canada. (1996). Royal commission report on Aboriginal peoples, volume 1. Retrieved from http://www.ainc-inac.gc.ca/ap/rrc-eng.asp.

Isaac T. (1995). An introduction to Aboriginal issues. Journal of the Society of Obstetricians \& Gynaecologists of Canada, 17, 583-585.

Issacs, A. N., Pyett, P., Oakley-Browne, M. A., Gruis, H., \& Waples-Crowe, P. (2010). Barriers and facilitators to the utilization of adult mental health services by Australia's Indigenous people: Seeking a way forward. International Journal of Mental Health Nursing, 19, 7582. $10.1111 / \mathrm{j} .1447-0349.2009 .00647 . \mathrm{x}$

Isaak, C. A. \& Marchessault, G. (2008). Meaning of health: The perspectives of Aboriginal adults and youth in a northern Manitoba First Nations community. Canadian Journal of Diabetes, 32, 114-132.

Israel, B. A., Schulz, A. J., Parker, E. P., Becker, A. B. (2001). Community-based participatory research: Policy recommendations for prompting a partnership approach in health research. Journal Education for Health, 14, 182-197.

Israel, B., Schulz, A., Parker, E., Becker, A. B. (1998). Review of community-based research: assessing partnership approaches to improve public health. Annual Review of Public Health 19, 173-94.

Jack, S. M., Brooks, S., Frugal, C. M., \& Dobbins, M. (2010). Knowledge transfer and exchange processes for environmental health issues in Canadian Aboriginal communities. International Journal of Environmental Research and Public Health, 7, 651-674.

Jacklin, K., \& Kinoshameg, P. (2008). Developing a participatory Aboriginal health research project: "Only if it's going to mean something". Journal of Empirical Research on Human 
Research Ethics, 3, 53-67. doi: 10.1525/jer.2008.3.2.53.

Jiwa, A., Kelly, L., \& St. Pierre-Hansen, N. (2008). Healing the community to heal the individual: Literature review of aboriginal community-based alcohol and substance abuse programs. Canadian Family Physician, 54, 1000.e1-7.

Jost, J. T., \& Kruglamski, A. W. (2002). The estrangement of social constructionism and experimental social psychology: History of the rift and prospect for reconciliation. Personality and Social Psychology Review, ^, 168-187.

Kagitcibasi, C. (2005). Autonomy and Relatedness in Cultural Context: Implications for Self and Family. Journal of Cross-Cultural Psychology, 36, 403-422. doi: $10.1177 / 0022022105275959$

Kaplan-Myrth, N. \& Smylie, J. (2006). Sharing what we know about living a good life. Regina, SK: First Nations University of Canada.

Kelly, P. (2005). Practical suggestions for community interventions using participatory action research. Public Health Nursing, 22 (1), 65-73. doi: 10.1111/j.0737-1209.2005.22110.x

Kessler, R. C., Sonnega, A., Bromet, E., Hughes, M., \& Nelson, C.B. (1995). Posttraumatic stress disorder in the National Comorbidity Survey. Archives of General Psychiatry, $52,1048-1060$.

Kirmayer, L. J. (1994). Suicide among Canadian Aboriginal Peoples. Transcultural Psychiatric Research Review, 31, 3-58.

Kirmayer, L. J., Fletcher, C., Corin, E., \& Boothroyd, L. (1997). Inuit concepts of mental health and illness: An ethnographic study. Montreal, QC: Institute of Community \& Family Psychiatry.

Kirmayer, L. J., Simpson, C., \& Cargo, M. (2003). Healing traditions: Culture, community and 
mental health promotion with Canadian Aboriginal peoples. Australian Psychiatry, 11, S15-S23.

Kral, M. J. (2009). Transforming communities: Suicide, relatedness, and reclamation among Inuit of Nunavut. Dissertation Abstracts International: Section A. Humanities and Social Sciences, 71(3-A), 991.

Krupnick, J. L., Sotsky, S. M., Simmens, S., Moyer, J., Elkin, I., Watkins, J., \& Pilkonis, P. A. (1996). The role of the therapeutic alliance in psychotherapy and pharmacotherapy outcome: Findings in the National Institute of Mental Health Treatment of Depression Collaborative Research Program. Journal of Consulting and Clinical Psychology, 64, $532-539$.

Lather, P. \& Smithies, C. (1997). Troubling the angels: Women living with HIV/AIDS. Boulder, CO: WestviewPress.

Lavallee, L. (2009). Practical application of an Indigenous research framework and two qualitative Indigenous research methods: Sharing circles and Anishnaabe symbol-based reflection. International Journal of Qualitative Methods, 8(1), 21-40.

Little Bear, L. (2000). Jagged world views colliding. In M. Battiste (Ed.), Reclaiming Indigenous voices and vision. (pp. 77-85). Vancouver, BC: University of British Columbia Press.

Martell, C. R., Dimidjian, S., \& Herman-Dunn, R. (2010). Behavioral activation for depression: A clinicians guide. New York, NY: Guildford Press.

MacCaulay, A. C., Paradis, G., Potvin, L., \& Cross, E. J. (1997). The Kahnawake Schools diabetes prevention project: Intervention, evaluation, and baseline results of a diabetes 
primary prevention program with native community in Canada. Prevention Medicine, 26, 779-790. doi: 10.1006/pmed.1997.0241

Manitoba Office of the Children's Advocate. (2003). Pauingassi First Nation report on solvent abuse. In D. O'Brien (Ed.) Manitoba sniff crisis has given birth to tragic trend... Babies smell like gas (pp. A1-A2). Manitoba: Winnipeg Free press.

Maracle, S. (2011). Consultation on Sexual Violence and Aboriginal Community. Ottawa, ON: Catalyst Research and Communications.

Martin, D. J., Garkse, J. P., \& Davis, M. K. (2000). Relation of the therapeutic alliance with outcome and other variables: A meta-analytic review. Journal of Consulting and Clinical Psychology, 68, 438-450.

McCormick, R. M. (2000). Aboriginal traditions in the treatment of substance abuse. Canadian Journal of Counselling, 34 (1), 25-32.

McKeough, A., Bird, S., Tourigny, E., Romaine, A., Graham, S., Ottmann, J., \& Jeary, J. (2008). Storytelling as a foundation to literacy development for Aboriginal children: Culturally and developmentally appropriate practices. Canadian Psychology, 49, 148-154.

McShane, K. E., Smylie, J. K., Hastings, P. D., Martin, C. M. \& Tungasuvvingat Inuit Family Resource Centre. (2006). Guiding health promotion efforts with urban Inuit: A communityspecific perspective on health information sources and dissemination strategies. Canadian Journal of Public Health, 97, 296-299.

Mendelssohn, F. D. (2008). Transgenerational transmission of trauma: Guilt, shame, and the "heroic dilemma". International Journal of Group Psychotherapy, 58, 389-401

Mental Health Commission of Canada. (2009). Toward recovery \& well-being: A framework for a mental health strategy for Canada. Calgary, AB: Mental Health Commission of Canada. 
Métis Nation of Ontario. (2010). Métis Nation of Ontario. Retrieved from http://www.metisnation.org/.

Minkler, M. (2000). Using participatory action research to build healthy communities. Public Health Reports, 115, 191-197.

Morgan, R \& Freeman, L. (2009). Healing of our people. Substance abuse and historical trauma. Substance Use and Misuse, 44 (1), 84-98. doi: 10.1080/10826080802525678

Nagata, D. K. (1991). The transgenerational impact of Japanese American internment: Clinical issues in working with the children of former internees. Psychotherapy, 28, 121-128.

Nagata, D. K. (1993). Legacy of injustice: Exploring the cross-generational impact of the Japanese American internment. New York, NY: Plenum Press.

Nagata, D. K. (1998). Intergenerational effects of Japanese American internment. In Y. Danieli (Ed.), International handbook of multigenerational legacies of trauma (pp. 125-139). New York, NY: Plenum Press.

National Aboriginal Community Controlled Health Organisation (1993). NACCHO Manifesto on Aboriginal Well-being. Canberra: NACCHO.

National Aboriginal Healing Organization. (2009). Cultural safety and knowledge sharing: Work on Mental Wellness at Inuit Tuttarvingat, NAHO. Poster retrieved from http://www.naho.ca/publications/topics/mental-health-and-addictions/?submit=view

Napolean, V. (2005). Aboriginal self determination: Individual self and collective selves. Atlantis, 29, 1-21.

Nelson, S. (2012). Challenging hidden assumptions: Colonial norms as determinants of Aboriginal mental health. Prince George, BC: National Collaboration Centre for Aboriginal Health. 
Nightingale, D. J., \& Cromby, J. (2002). Social constructionism as ontology. Theory \& Psychology, 12, 701-713.

Norris, M. J. (2007). Aboriginal languages in Canada: Emerging trends and perspective on second language acquisition. Ottawa, ON: Statistics Canada.

Nunavut Bureau of Statistics. (2003). Statistics on death by suicide in Nunavut. Iqaluit, NU: Government of Nunavut.

O’Connor, K. (2010). Experiential Learning in an Indigenous Context: Integration of place, experience and criticality in educational practice. Canadian Council of Learning.

Ontario Curriculum (2005). Aboriginal perspectives: The teacher's toolkit. Toronto, ON: Ontario Ministry of Education.

Ontario Federation of Indigenous Friendship Centres (2013). Ontario Federation of Indigenous Friendship Centres. Retrieved from http://www.ofifc.org/.

Orford, J. (1992). Community psychology: Theory and practice. Chichester: Wiley.

Paul, K-L. (Producer). (2012, December 3). Idle No More Alberta - Dr. Pamela Palmater (Ppart 3 of 4). Retrieved from https://www.youtube.com/watch?v=s4ku8vVELYs

Petawabano, B., Gourdeau, E., Jourdain, F., Palliser-Tulugak, A., \& Cossette, J. (1994). Mental health and Aboriginal people of Quebec. Montreal: Gaetan Morin Editeur.

Poonwassie, A., \& Charter, A. (2001). An Aboriginal worldview of helping: Empowering approaches. Canadian Journal of Counselling, 35(1), 63-73.

Potter, J., \& Wetherell, M. (1987). Discourse and social psychology: Beyond attitudes and behavior. London, UK: SAGE Publications Ltd.

Potts, A., Gavey, N., Grace, V. M., \& Vares, T. (2003). The downside of Viagnara: Women's experiences and concerns. Sociology of Health \& Illness, 25, 697-719. 
Ranford, J., \& Warry, W. (2006). Indigenous health research knowledge transfer/translation network. Hamilton, ON: Indigenous Health Research Development Program.

Reilly, R. E., Doyle, J., Bretherton, D., \& Rowley, K. G. (2008). Identifying psychosocial mediators of health amongst Indigenous Australians for the Heart Health Project. Ethnicity \& Health, 13, 351-373.

Ryerson University (2013). Ryerson Mental Health Advisory Committee Report. Toronto, ON: Ryerson University.

Sack, W., Beiser, M., Baker-Brown, G., \& Redshirt, R. (1994). Depressive and suicidal 2004) symptoms in Indian school children: Some findings from the Flower of Two Soils. American Indian and Alaska Native Mental Health Research, 4, 81-96.

Schnarch, B. \& First Nations Centre, \& National Aboriginal Health Organization (2004).

Ownership, control, access and possession (OCAP) or self-determination applied to research: A critical analysis of contemporary First Nations research and some options for First Nations communities. Journal of Aboriginal Health, 1, 80-95.

Shannon, K., Spittal, P., \& Thomas, V. (2007). Intersections of trauma, substance use, and HIV vulnerability among Aboriginal girls and young women who use drugs: In N., Poole \& L. Greaves (Ed.). Highs \& Lows. Canadian Perspectives on Women and Substance Use. (pp. 169-175). Toronto: Centre for Addiction and Mental Health.

Sinclair, C., \& Pettifor, J. (2001). Companion manual to the Canadian code of ethics for psychologists, third edition. Ottawa, ON: Canadian Psychological Association.

Smith, D., Varcoe, C., \& Edwards, N. (2005). Turning around the intergenerational impact of residential schools on Aboriginal people: Implications for health policy and practice. Canadian Journal of Nursing Research, 37, 38-60. 
Smylie, J. (2000). SOCG Policy Statement. A guide for health professionals working with Aboriginal peoples: the sociocultural context of Aboriginal peoples in Canada. Journal of the Society of Obstetricians and Gynaecologists of Canada, 22, 1070-81.

Smylie, J. (2001). SOCG Policy Statement. A guide for health professionals working with Aboriginal peoples: Health issues affecting Aboriginal peoples. Journal of the Society of Obstetricians and Gynecologists of Canada, 23, 54-68.

Smylie, J., Kaplan-Myrth, N., McShane, K., Métis Nation of Ontario - Ottawa Council, Pikakanagan First Nation, Tungasuvvingat Inuit Family Resource Centre. (2009). Indigenous knowledge translation: Baseline findings in a qualitative study of the pathways of health knowledge in three Indigenous communities in Canada. Health Promotion Practice, 10, 436-446. doi: 0.1177/1524839907307993

Smylie, J., Martin, C.M., Kaplan-Myrth, N., Steele, L., Tait, C., Hogg, W. (2003). Knowledge translation and indigenous knowledge. International Journal of Circumpolar Health, 63 (Suppl 2), 139-143.

Smye, V., \& Mussell, B. (2001). Aboriginal mental health: 'What works best'. British Columbia: Mental Health Evaluation and Community Consultation Unit.

Sobh, R., \& Perry, C. (2005). Research design and data analysis in realism research. European Journal of Marketing, 40, 1194-1209.

Soul of the Mother (n.d.). Soul of the mother: Seeds of the spirits. Retrieved from http://www.soulofthemother.org.

Spiwak, R., Sareen, J., Elias, B., Martens, P., Munro, G., Bolton, J. (2012). Complicated grief in Aboriginal populations. Dialogues in Clinical Neurosciences, 14, 204-209.

Statistics Canada. (2001). 2001 Census Dictionary. Retrieved from 
http://www12.statcan.ca/english/census01/Products/Reference/dict/index.htm\#dictionary.

Statistics Canada. (2003). Aboriginal peoples of Canada: A demographic profile 2001 Census: Analysis Series. Ottawa, ON: Statistics Canada.

Statistics Canada. (2006). Aboriginal Peoples in Canada in 2006: Inuit, Métis and First Nations, 2006 census. Ottawa, ON: Statistics Canada

Stewart, S. (2011). Indigenous research methods and healing. International Journal of Health Promotion and Education, 12, 15-28.

Stone Circle. (2011). Research on effective practice to support Métis learners achievement and self-identification project. Ottawa, ON: Métis Nations of Ontario.

Sudsawad, P. (2007). Knowledge translation: Introduction to models, strategies, and measures. Wisconsin, USA: The National Center for the Dissemination of Disability Research. Taylor, S. (2001). Evaluating and applying discourse analytic research. In M. Wetherell, S. Taylor \& S. J. Yates (Eds.), Discourse as data: A guide for analysis. London: Sage. Teasdale, K. E., Conigrave, K. M., Kiel, K, A., Freeburn, B., Long, G., \& Becker, K. (2008). Improving services for prevention and treatment of substance misuse for Aboriginal communities in a Sydney area health service. Drug and Alcohol Review, 27, 152-159. doi: $10.1080 / 09595230701829447$

The First Nations Information Governance Centre. (2012). First Nations Regional Health Survey (RHS) Phase 2 (2008/10) National Report for Adults, Youth and Children Living in First Nations Communities. Ottawa, ON: The First Nations Information Governance Centre. Towle, A., Godolphin, W., \& Alexander, T. (2006). Doctor-patient communications in the Aboriginal community: Towards the development of educational programs. Patient Education and Counselling, 62, 340-346. doi: 10.1016/j.pec.2006.06.006 
Truth and Reconciliation Commission of Canada (n.d.). Meet the commissioners. Retrieved from http://www.trc.ca/websites/trcinstitution/index.php?p=5.

Vasquez, M. J. T. (2007). Cultural difference and the therapeutic alliance: An evidence-based analysis. American Psychologist, 62, 878-885. doi: 10.1037/0003-066X.62.8.878

Vicary, D., \& Westerman, T. (2004). 'That's just the way he is': Some implication of Aboriginal mental health beliefs. Australian eJournal for the Advancement of Mental Health, 3, 1-10.

Vinkle, E. (2012). Cultural competency - working with Aboriginal peoples: A non-Native perspective. Native Social Work, 8, 129-142.

Wallerstein, N., \& Duran, B. (2003). Chapter 2: The conceptual, historical and practice roots of community based participatory research and related participatory traditions. In M. Minkler \& N. Wallerstein (Eds). Community Based Participatory Research for Health. (pp. 27-52). San Francisco, CA: Jossey-Bass.

Wilson, H. (2007). N’tacimowin inna nah': Coming in to two-spirit identities (Doctoral dissertation). Retrieved from PsycINFO.

Wilson, K. M., Brady, T. J., \& Lesesne, C. (2011). An organizing framework for translation in public health: The knowledge to action framework. Preventing Chronic Disease, 8, A46.

Wood, N. B. (2000). The sacred pipe. Sacred Hoop, 31, 19-21.

World Health Organization. (2002). World report on violence and health. PDF Document. Retrieved from: http://www.who.int/violence_injury_prevention/violence/world_report/en/.

World Health Organization. (2011). Social determinants of health: Key concepts. Retrieved from http://www.who.int/social_determinants/thecommission/finalreport/key concepts/en/ 